Edith Cowan University

Research Online

$1-1-2003$

\title{
In pursuit of best practice : Benchmarking tools and processes for the management of hazardous substances in the workplace
}

\author{
Barry Chesson \\ Edith Cowan University
}

Follow this and additional works at: https://ro.ecu.edu.au/theses

Part of the Occupational Health and Industrial Hygiene Commons

\section{Recommended Citation}

Chesson, B. (2003). In pursuit of best practice : Benchmarking tools and processes for the management of hazardous substances in the workplace. https://ro.ecu.edu.au/theses/1300

This Thesis is posted at Research Online.

https://ro.ecu.edu.au/theses/1300 


\section{Edith Cowan University}

\section{Copyright Warning}

You may print or download ONE copy of this document for the purpose of your own research or study.

The University does not authorize you to copy, communicate or otherwise make available electronically to any other person any copyright material contained on this site.

You are reminded of the following:

- Copyright owners are entitled to take legal action against persons who infringe their copyright.

- A reproduction of material that is protected by copyright may be a copyright infringement. Where the reproduction of such material is done without attribution of authorship, with false attribution of authorship or the authorship is treated in a derogatory manner, this may be a breach of the author's moral rights contained in Part IX of the Copyright Act 1968 (Cth).

- Courts have the power to impose a wide range of civil and criminal sanctions for infringement of copyright, infringement of moral rights and other offences under the Copyright Act 1968 (Cth). Higher penalties may apply, and higher damages may be awarded, for offences and infringements involving the conversion of material into digital or electronic form. 
In Pursuit of Best Practice: Benchmarking Tools and Processes for the Management of Hazardous Substances in the Workplace

Barry Chesson B.Sc (Hons), Grad. Dip. Nat. Res., M.App.Sc. (Health Science), $\mathrm{ClH}$, C.Chem., C.P.Chem., FRACl, MAIOH. 


\section{USE OF THESIS}

The Use of Thesis statement is not included in this version of the thesis. 


\begin{abstract}
In Pursuit of Best Practice: Benchmarking Tools and Processes for the Management of Hazardous Substances in the Workplace
\end{abstract}

Many organisations now strive to achieve excellence in various aspects of occupational health and safety. Benchmarking of the techniques and approaches of other organisations is becoming a popular way of bridging gaps and seeking to achieve high levels of performance. There exist many sources of guidance in the form of external and internal standards, regulations, codes of practice, publications by professional institutions and similar. However, there are clear shortfalls in terms of tools and processes needed to identify areas of opportunity and to overcome barriers to the efficient transfer of ideas and techniques from one enterprise to another. This is true for all organisations, but particularly so for small/medium sized facilities with limited resources and expertise.

This study has sought to develop and test new tools and processes to make benchmarking activity and the transfer of technology, ideas and approaches more efficient and meaningful. it has drawn heavily from state-of-the-art management theory and has sought to establish the linkage between the people factor, the workplace environment factor and the organisation of work factor as they contribute to workplace health and safety performance. It has used qualitative inquiry methodologies and an approach based on personal contact and insight, as expressed by Patton (1990, p. 46), to generate data. The fieldwork component of the study was conducted at eight mining, mineral processing and related industry sites within Western Australia. The subject of the study was the facility's processes and practices in regard to the management of hazardous materials. This was chosen partly because chemical-induced injury and disease remain a significant problem for workers in industry (Winder, $1999 \mathrm{~b}$, p. 168) and partly because of its complexity and degree of difficulty. Data collection was based on the three qualitative inquiry methods, namely in-depth, open-ended interviews with the Site Manager 
and the Site Occupational Health and Satety (OHS) Professional, direct observation and review of written documents.

Also tested was the assumption that if the materials developed during the study can be applied successfully in the area of hazardous materials, then other less complex areas under the OHS umbrella could be approached with confidence.

There is potential for the tools and processes developed and evaluated in this work to be used widely in the transfer of best practice, that is, to be deployed beyond the hazardous substances focus of this study and beyond the Mining Industry of Western Australia. Study outcomes and the new materials that have been generated will assist with the selection of benchmarking partners and will help to identify "pockets of excellence" for focused attention. This will encourage and assist organisations to take steps towands identifying and implementing Industry best practice in the element of interest. There is potential for study outcomes to impact positively on OHS practices within many organisations - and thereby to reduce the personal and societal cost of injury and illness outcomes associated with the use of hazardous materials at work. 


\section{Declaration}

I certify that that this thesis does not, to the best of my knowledge and belief:

(i) incorporate without acknowledgment any material previously submitted for a degree or diploma in any institution of higher education;

(ii) contain any material previously published or written by another person except where due reference is made in the text; or

(iii) contain any defamatory material. 


\section{Acknowledgments}

The author would like to acknowledge the encouragement and advice provided by his Supervisor, Associate Professor Milos Nedved, and Associate Supervisor, Dr Janis Jansz, during the course of this study.

Many people lent assistance during the data gathering stage. These include Mr Greg Yarrick, Mr Brian Peoples, Ms Janie Dumbrell, Ms Louise Coubrough, Ms Johanne Marias, Ms Deborah Leonard, Dr Nic Ormonde, Ms Pierina Otness, Ms Karen Racco, Mr Dave Bies, Mr Paul Harring, Mr David Smith, Ms Julie Budrey, Ms Marie-lize de Bruyn, Mr Martin Knee, Mr Grant Robinson, Mr Trevor Taylor and Mr Jack Farry.

Thanks are also directed to the Management and Staff of Alcoa of Australia Limited for on-going support and commitment to all matters bearing on the health and safety of employees. The continuous improvement environment that prevails within Alcoa fosters innovation and achievement on OHS and there prevails a shared vision of zero injuries and ill health. 
Copyright and access

Titte page

Abstract

Declaration

Acknowledgments

CHAPTER 1 INTRODUCTION

1.1 Background to the study' 21

1.2 Significance of the study 23

1.3 Scope of the study 27

$\begin{array}{lll}1.4 & \text { Purpose of the study } & 27\end{array}$

1.5 Research questions 28

1.6 Operational definitions 28

1.7 Sources of information 32

1.8 Document structure 33

\section{CHAPTER 2 REVIEW OF THE LITERATURE}

2.1 The Quality Management approach

2.1.1 Background, concepts and development 35

2.1.2 Culture and change 38

2.1.3 Tools and processes $\quad 39$

2.1.4 Impact on management systems 42

2.1.5 Impact on OHS 43

2.2 Legal perspectives

2.2.1 Background 46

2.2.2 Developments and trends - international 48

2.2.3 Developments and trends - Australia 49

2.2.4 Developments and trends - Western Australia $\quad 52$ 
2.3 OHS management

2.3.1 Background 54

2.3.2 Conceptual framework 57

2.3.3 OHS and organisational effectiveness $\quad 59$

2.3.4 Management of change 64

2.3.5 Management systems and related
standards

2.3.6 Behavioliral safety $\quad 71$

2.3.7 Risk management $\quad \therefore \quad: \quad 86$

2.3.8 Hazard identification $\quad 88$

2.3.9 Risk assessment $\quad 89$

2.3.10 Risk control 93

2.3.11 Implementation issues $\quad 96$

2.4 Hazardous materials management

2.4.1 Hazardous materials - strategic planning and process $\quad 98$

2.4.2 Components of a facility program 99

2.4.3 A fishbone for chemical safety 100

2.4.4 Implementation issues $\quad 101$

2.5 The search for best practice

2.5.1 The concept 102

2.5.2 Performance measurement - concepts,
processes and tools
2.5 .3 Peformance

2.5.3 Performance reporting and review 115

2.5.4 Audits - concepts, processes and tools 118

2.5.5 Benchmarking - concepts, processes
and tools

2.5.6 Technology transfer - implementation
issues

2.6 Research design and thesis preparation $\quad 140$

2.7 Summary 141

\section{CHAPTER 3 MATERIALS AND METHODS}

3.1 Research methodology

3.2 Target population

3.2.1 Facilities

3.2 .2 Individuals 144

3.3 Study design

3.3.1 Addressing the research questions 145

3.3.2 Study sequence 147

3.4. Input model 147

3.5 Development of tools and methodologies 148 
3.6 Data collection, analysis and communication procedures.

3.7 Reliability and validity of study processes and tools

3.7.1 Reliability

3.7.2 Validity

3.8 Deliverables for participating organisations

3.9 Supplementary exercise - transferability

3.10 Limitations

\section{CHAPTER 4 RESULTS}

4.1 Overview

4.2 Facility One

4.2.1 The operation

4.2.2 The workforce

4.2.3 Organisation for OHS.

4.2.4 Principal OHS issues and challenges

4.2.5 Other relovant information

4.2.6 Organisational effectiveness

168

4.2.7 Benchmarking, best practice and technology transfer

4.2.8 Self-ranking in O'tS

4.2.9 Potential benchmarking partners

4.2.10 Chemical safety preliminary questionnaire

4.2.11 Flow chart

4.2.12 Critical success factors

4.2.13 Performance measurement

4.2.14 Innovations in chemical safety

4.2.15 Compliance

4.2.16 Organisation of work

4.2.17 Hazard recognition

4.2.18 Risk assessment

4.2.19 Risk control

4.2.20 Industrial Hygiene program development

4.2.21 People initiatives

4.3 Facility Two

4.3.1 The operation

4.3.2 The workforce

4.3.3 Organisation for $\mathrm{OHS}$

4.3.4 Principal OHS issues and challenges

189

4.3.5 Other relevant information 
4.3.6 Organisational effectiveness

4.3.7 Benchmarking, best practice and technology transfer

4.3.8 Self-ranking in OHS

4.3.9 Potential benchmarking partners

4.3.10 Chemical safety preliminary questionnaire

4.3.11 Flow chart

4.3.12 Critical success factors

4.3.13 Performance measurement

4.3.14 Innovations in chemical safety

196

4.3.15 Compliance

196

4.3.16 Organisation of work

199

4.3.17 Hazard recognition

203

4.3.18 Risk assessment

204

4.3.19 Risk control

204

4.3.20 Industrial Hygiene program development

207

4.3.21 People initiatives

208

4.3.22 Summary

210

4.4 Facility Three

4.4.1 The operation

211

4.4.2 The workforce 211

4.4.3 Organisation for OHS 212

4.4.4 Principal OHS issues and challenges 213

4.4.5 Other relevant information 213

4.4.6 Organisational effectiveness 214

4.4.7 Benchmarking, best practice and technology transfer

4.4.8 Self-ranking in OHS

215

4.4.9 Potential benchmarking partners

215

4.4.10 Chemical safety preliminary questionnaire $\quad 215$

4.11 Flow chart

4.4.11 Flow chart

216

4.4.12 Critical success factors $\quad 218$

4.4.13 Performance measurement : 218

4.4.14 Innovations in chemical safety 218

4.4.15 Compliance 219

4.4.16 Organisation of work , 220

4.4.17 Hazard recognition 223

4.4.18 Risk assessment $\quad 224$

4.4.19 Risk control 225

4.4.20 Industrial Hygiene program development 226

4.4.21 People initiatives $\quad 227$

4.4.22 Summary 228

4.5 Facility Four

4.5.1 The operation 230

4.5.2 The workforce 230

4.5.3 Organisation for OHS 231

4.5.4 Principal OHS issues and challenges 232

4.5.5 Other relevant information 232

$\begin{array}{ll}4.5 .6 & \text { Organisational effectiveness } \quad 232\end{array}$ 
4.5.7 Benchmarking, best practice and technology transfer

4.5.8 Self-ranking in OHS 233

4.5.9 Potential benchmarking partners 233

4.5.10 Chemical safety preliminary questionnaire 233

4.5.11 Flow chart 234

4.5.12 Critical success factors 236

4.5.13 Performance measurement 236

4.5.14 Innovations in chemical safety 236

4.5.15 Compliance $\quad .237$

4.5.16 Organisation of work 238

4.5.17 Hazard recognition 242

4.5.18 Risk assessment 243

4.5.19 Risk control 244

4.5.20 Industrial Hygiene program development 245

4.5.21 People initiatives $\quad 246$

4.5.22 Summary 247

4.6 Facility Five

4.6.1 The operation 248

4.6.2 The workforce 250

4.6.3 Organisation for OHS $\quad 250$

4.6.4 Principal OHS issues and challenges 250

4.6.5 Other relevant information 250

4.6.6 Organisational effectiveness 251

$\begin{array}{ll}\text { 4.6.7 Benchmarking, best practice and } & \\ \text { technology transfer } & 252\end{array}$

4.6.8 Self-ranking in OHS 252

4.6.9 Potential benchmarking partners 252

4.6.10 Chemical safety preliminary questionnaire 252

4.6.11 Flow chart 253

4.6.12 Critical success factors $\quad 255$

4.6.13 Performance measurement 255

4.6.14 Innovations in chemical safety $\quad 255$

4.6.15 Compliance 256

4.6.16 Organisation of work 257

4.6.17 Hazard recognition $\quad 260$

4.6.18 Risk assessment $\quad 261$

4.6.19 Risk control $\quad \therefore \quad 262$

4.6.20 Industrial Hygiene program development $\quad 264$

4.6.21 People initiatives $\quad 264$

4.6.22 Summary $\quad 265$

4.7 Facility Six

4.7.1 The operation 267

4.7.2 The workforce 268

4.7.3 Organisation for OHS 269

4.7.4 Principal OHS issues and challenges 269

4.7.5 Other relevant information 270

$\begin{array}{ll}4.7 .6 & \text { Organisational effectiveness } \\ & 270\end{array}$

4.7.7 Benchmarking, best practice and 
technology transfer 271

4.7.8 Self-ranking in OHS 271

4.7.9 Potential benchmarking partners 272

4.7.10 Chemical safety preliminary questionnaire : $\quad 272$

4.7.11 Flow chart $\quad 273$

4.7.12 Critical success factors $\quad 275$

4.7.13 Performance measurement : $\quad 275$

4.7.14 innovations in chemical safety 275

4.7.15 Compliance 276

4.7.16 Organisation of work 278

4.7.17 Hazard recognition $\quad 282$

4.7.18 Risk assessment $\quad 282$

4.7.19 Risk control 283

4.7.20 Industrial Hygiene program development $\quad 284$

4.7.21 People initiatives 284

$\begin{array}{ll}4.7 .22 & \text { Summary } \quad 287\end{array}$

4.8 Facility Seven

4.8.1 The operation 288

4.8.2 The workforce 290

4.8.3 Organisation for OHS 290

4.8.4 Principal OHS issues and challenges 290

4.8.5 Other relevant information 291

4.8.6 Organisational effectiveness 291

$\begin{array}{ll}\text { 4.8.7 Benchmarking, best practice and } & \\ \text { technology transfer } & 292\end{array}$

4.8.8 Self-ranking in OHS 292

4.8.9 Potential benchmarking partners 292

4.8.10 Chemical safety preliminary questionnaire 293

4.8.11 Flow chart 293

4.8.12 Critical success factors 295

4.8.13 Performance measurement 295

4.8.14 Innovations in chemical safety 295

4.8.15 Compliance $\quad 296$

4.8.16 Organisation of work $\quad 297$

4.8.17 Hazard recognition $\quad 299$

4.8.18 Risk assessment $\quad 300$

4.8.19 Risk control 301

4.8.20 Industrial Hygiene program development 302

4.8.21 People initiatives 303

4.8.22 Summary 303

4.9 Facility Eight

4.9.1 The operation 305

4.9.2 The workforce 306

4.9.3 Organisation for OHS 307

4.9.4 Principal OHS issues and challenges . 307

4.9.5 Other relevant information 308

4.9.6 Organisational effectiveness 308

4.9.7 Benchmarking, best practice and
technology transfer 
4.9.8 Self-ranking in OHS $\quad 309$

4.9.9 Potential benchmarking partners 309

4.9.10 Chemical safety preliminary questionnaire $\quad 310$

4.9.11 Flow chart 311

4.9.12 Critical success factors $\quad 312$

4.9.13 Performance measurement $\quad$. $\quad 313$

4.9.14 Innovations in chemical safety 313

4.9.15 Compliance 313

4.9.16 Organisation of work 315

4.9.17 Hazard recognition $\quad 318$

4.9.18 Risk assessment $\quad 318$

4.9.19 Risk control $\quad 319$

4.9.20 Industrial Hygiene program development 321

4.9.21 People initiatives 321

4.9.22 Summary 322

4.10 The supplementary exercise - transferability 324

$\begin{array}{ll}4.11 \text { Summary } & 325\end{array}$

\section{CHAPTER 5 DISCUSSION}

5.1 Overview

5.2. Research methodologies and tools

5.2.1 Influencing factors in the literature 326

5.2.2 Design aspects 330

5.2.3 Research processes $\quad 330$

5.2.4 Research tools $\quad 332$

5.2.5 Assumptions 332

5.3 Intra-site observations and comparisons
5.3.1 Facility One

5.3.2 Facility Two 336

5.3.3 Facility Three $\quad 338$

5.3.4 Facility Four $\quad 340$

5.3.5 Facility Five $\quad 341$

5.3.6 Facility Six 343

5.3.7 Facility Seven $\quad 345$

$\begin{array}{ll}\text { 5.3.8 Facility Eight } & 347\end{array}$

5.4 Inter-site observations and comparisons

5.4.1 Diversity 349

5.4.2 Booklet One material 349

5.4.3 Booklet Two material $\quad 350$

5.4.4 Booklet Three material 354

5.4.5 Booklet Four material 354

5.4.6 Booklet Five material 356

5.4.7 Booklat Six material $\quad 357$

5.4.8 Scoring and relationships between variables $\quad 359$ 
5.5 Addressing the research questions

5.6 Summary

\section{CHAPTER 6 CONCLUSIONS AND RECOMMENDATIONS}

6.1 Conclusions

6.1.1 Study objectives and outcomes

6.1.2 Novel aspects of the study

6.1.3 Usefulness of this information

6.2 Recommendations

6.2.1 New frontiers and prospects for further work

6.2.2 Next steps

APPENDIX 1 Scope and functions of Industrial Hygiene

APPENDIX 3 Performance reporting and review

- The safety dashboard

- The safety performance index

- The balanced scorecard

APPENDIX 4 Powerpoint presentation package - study proposals

- Booklet 1: Site profile

- Booklet 2: Preliminary questions 
- Booklet 3: Compliance $\$ 36$

- Booklet 4: Organisation of work 444

- Booklet 5: The workplace environment 452

- Booklet 6: People initiatives $\quad 460$

- Booklet 7: Results: site strengths and opportunities for improvement 464

- Booklet 8: Scoresheets, inter-site comparisons and benchmarking opportunities

APPENDIX 8 Sample report to site management 480

APPENDIX 9 Supplementary exercise on transferability:

A booklet for ergonomics

APPENDIX 10 Benchmarking opportunities 505

- Benchmarking opportunities - OHS (general) 506

- Benchmarking opportunities - Chemical Safety 508 


\section{LIST OF TABLES}

Table 1 Qualitative measures of consequence

Table 2 Qualitative measures of likelihood

Table 3 Qualitative risk analysis matrix: consequence and likelihood

92

Table 4 Qualitative risk analysis matrix: risk rankings and actions

92

Table $5 \quad$ Organisation of work - Facility One

176

Table 6 Hazard recognition: Facility One

179

Table $7 \quad$ Risk assessment: Facility One

181

Table $8 \quad$ Risk control: Facility One

182

Table $9 \quad$ People initiatives: Facility One

185

Table 10 Organisation of work: Facility Two

200

Table 11 Hazard recognition: Facility Two

203

Table 12 Risk assessment: Facility Two

205

Table $13 \quad$ Risk control: Facility Two

206

Table 14: $\quad$ People initiatives: Facility Two

209

Table 15 Organisation of work: Facility Three

221

Table 16 Hazard recognition: Facility Three

224

Table 17 Risk assessment: Facility Three

225

Table 18 Risk control: Facility Three

226 


\section{LIST OF TABLES (CONT.)}

Table 19 People initiatives: Facility Three

Table $20 \quad$ Organisation of work: Facility Four

Table $21 \quad$ Hazard recognition: Facility Four

Table 22 Risk assessment: Facility Four

Table $23 \quad$ Risk control: Facility Four

Tabie $24 \quad$ People initiatives: Facility Four

Table 25 Organisation of work: Facility Five

Table 26 Hazard recognition: Facility Five

Table 27 Risk assessment: Facility Five

Table 28

Risk control: Facility Five

263

Table 29

$$
\text { People initiatives: Facility Five }
$$

Table $30 \quad$ Organisation of work: Facility Six

Table $31 \quad$ Hazard recognition: Façility Six

Table 32 Risk assessment: Facility Six

Table $33 \quad$ Risk control: Facility Six

Table $34 \quad$ People initiatives: Facility Six

Table $35 \quad$ Organisation of work: Facility Seven

Table $36 \quad$ Hazard recognition: Facility Seven

Table $37 \quad$ Risk assessment: Facility Seven 


\section{LIST OF TABLES (CONT.)}

Table $38 \quad$ Risk control: Facility Seven

Table $39 \quad$ People initiatives: Facility Seven

Table $40 \quad$ Organisation of work: Facility Eight

Table $41 \quad$ Hazard recognition: Facility Eight

Table 42 Risk assessment: Facility Eight

Table 43

Risk control: Facility Eight

Table 44

People initiatives: Facility Eight

322

Table 45

Focus group rating of ergoncmics booklet

324

Table 46

Number of identified benchmarking opportunities 


\section{LIST OF FIGURES}

Figure 1 A conceptual framework for OHS

Figure 2 Elements of successful OHS management

Figure 3 The ABC model of human behaviour

Figure 4 The risk management process

Figure 5 The hierarchy of control measures

Figure 6 A fishbone for chemical safety

Figure 7

A self-assessment audit process

Figure 8

The broader audit process

Figure 9

A flowchart for self-assessment deployment

Figure 10

A generic benchmarking process

Figure 11 An input model for benchmarking processes and tools

Figure 12 Schematic flowsheet - Bauxite to alumina (Facility One)

Figure 13 Acquisition of new chemicals - Facility One

Figure $14 \quad$ Facility profile - Facility One

Figure 15 Schematic flowsheet - Bauxite mining process (Facility Two)

Figure $16 \quad$ Acquisition of new chemicals - Facility Two

Figure $17 \quad$ Facility profile - Facility Two 


\section{LIST OF FIGURES (CONT.)}

Figure 18 Schematic of operational areas - Facility Three

Figure 19 Acquisition of new chemicals - Facility Three

Figure $20 \quad$ Facility profile - Facility Three

Figure 21 Schematic flowsheet - Extraction of gold-bearing ore (Facility Four)

Figure 22 Acquisition of new clemicals - Facility Four

Figure $23 \quad$ Facility profile - Facility Four

Figure 24 Schematic flowsheet - Ore to gold bullion (Facility Five)

Figure 25 Acquisition of new chemicals - Facility Five

Figure $26 \quad$ Facility profile - Facility Five

Figure 27 Schematic flowsheet - Open cut mining of hematite ore (Facility Six)

Figure 28 Acquisition of new chemicals - Facility Six

Figure $29 \quad$ Facility profile - Facility Six

Figure 30 Schematic flowsheet - Ore to gold bulion (Facility Seven)

Figure $31 \quad$ Acquisition of new chemicals - Facility Seven

Figure 32 Facility profile - Facility Seven

Figure 33 Schematic flowsheet - Nickel refining process (Facility Eight)

Figure 34 Acquisition of new chemicals - Facility Eight 


\section{LIST OF FIGURES (CONT.)}

Figure $35 \quad$ Facility profile - Facility Eight

Figure $36 \quad$ Make-up of facility scores

Figure 37

Innovation scores

Figure $38 \quad$ Innovation scores vs 2002 Lost Time Injury Frequency Rates

Figure 39

Innovation Scores vs DOIR audit scores

363

Figure 40

Legislative framework

402 


\section{CHAPTER 1 INTRODUCTION}

\subsection{Backgrounci to the study}

The past decade has seen many changes. The advent of the Information Age, changes in patterns of trade between nations, the rise in concern about global warming and the need to achieve a sustainable future for the planet have helped foster the concept that we are part of a global marketplace and that we live in a global village. Insular approaches are no longer deemed acceptable (National Industry Extension Service, 1993, p. 1).

In a Darwinian sense, organisations that cannot adapt to the new commercial and technological environment will not survive. Benchmarking the practices of successful organisations and adopting or adapting the best features of what is revealed is a way of achieving timely improvements and bridging the gaps (Blewett \& Shaw, 1995b, p. 237).

One of the critical performance areas for the modern organisation is environment, health and safety (EHS). For some, this is a priority area to rank with production, quality and cost. A select few have an approach that transcends this - EHS is embedded in their values. Since safety is a natural, shared value, progress in safety points to a learning organisation capable of achieving its wider commercial goals (Mussett, 2000, p. ii).

Within the EHS area, there are numerous physical, chemical and biological agents that are capable of impacting on the workforce, the cornmunity or the wider environment.

Hazardous chemicals represent one area under the EHS umbrella. The World Health Organisation (1998, p. 1) estimates that the total number of chemicals in the market place is currently about 100,000 and that the value of total global annual production is about 1.5 trillion US dollars. The International Labour Organisation (cited in Chamber of Commerce and Industry of WA, 1999, p. 1) claims that each year there are more than 1.1 
million deaths around the world that arise from workplace activities. Of these deaths, one-quarter result from exposure to hazardous substances that give rise to disabling illnesses such as cancer and cardiovascular, respiratory and nervous system disorders.

Health risks have a number of features that distinguish them from those in the safety area. The Health and Safety Executive (1992, p. 38) suggests the following:

- III health often results from complex biological processes, such as the repair of repeated damage (irritant dermatitis), immune responses (asthma), or abnormal cell behaviour (cancers) - as distinct from immediate injury.

- These processes may take place over extended periods. Thus, hazards may only become apparent after many people have been put at risk. Cases of disease may continue for decades after exposure has been controlled.

- The same disease may have both occupational and non-occupational causes. Asthma, back pain and lung cancer are examples of this.

- Exposure to disease risks is not always apparent and measurement of risk factors is often required.

The Minerva Institute (1991, p. A-218) put it that "occupational diseases, once rarely discussed, are now at the forefront because management is expected to shield employees from exposure to carcinogens, toxic substances, and other hazards known to cause disease or serious physical injury."

Winder (1999a, p. 99) and Hartley (2000, p. 8) refer to the use of more than 40,000 chemicals in Australian industry, with several hundred of these in widespread industrial use. Winder notes that these chemicals have often been used by workers in workplaces with little consideration of hazards or appropriate controls (Winder, 1999a, p. 100).

Generally, substances that may be described as toxic, corrosive, explosive, reactive, radioactive, flammable and similar should be considered hazardous substances (Malachowski, 1995, p. 5). The hazard 
presented by a substance has two components - firstly, the inherent ability of the substance to do harm and, secondly, the ease by which the substance can come in contact with the body (Malachowski, 1995, p. 4).

Industrial Hygiene $(\mathrm{IH})$ is the scientific discipline that encompasses the identification, evaluation and control of chemicals at work, and the techniques and approaches from this field are germane to this study. Appendix 1 provides detail on the scope and functions of Industrial Hygiene, as described by the American Board of Industrial Hygiene (1984).

Some organisations have excellent systems in place to manage chemicals and prevent any deleterious effects on people or the environment. Others fail to address hazardous materials in a satisfactory way. For them, a poor chemical safety record has an impact on overall performance and can severely limit an organisation's productivity (Winder, 1995, p. 212).

The advent of the Total Quality Management (TQM) phenomenon has provided a means of achieving high levels of performance. The TQM philosophy recognises that customer satisfaction, EHS considerations and business objectives are mutually dependent and are applicable in any organisation (Fisher, 1991, p. 23; Deacon, 1994, p. 20). The quality management philosophies, tools and approaches should be directly applicable to the management of hazardous substances. An approach that will be examined in depth in this research is that of benchmarking best practice in other organisations with a view to transfer and application of the findings to achieve higher levels of performance. As Watson (1992, p. 6) put it: "Benchmarking is a quality tool that helps set the direction for long-term, strategic business improvement".

\subsection{Significance of the study}

This study has drawn heavily from models, processes and tools that have emanated from the quality management area. $f$ has sought to develop and test new benchmarking tools and processes within the province of hazardous materials management. 
Hazardous materials management was chosen as the element to be focused on in this study. However, the element could have been mobile equipment operation, falls prevention, confined space hazards, electrical safety, contractor safely, hearing conservation, radiation protection, or any of dozens of other important topics within the Occupational Health and Safety (OHS) field. Hazardous materials had appeal in that chemicals are present in almost all workplaces, there is a vast number of chemical entities in existence, their acute and chronic effects are often not well understood, there is a certain aura or mystique about them, and they feature in many workplace mishaps.

Winder (1995, p. 212) has reviewed a range of studies looking at aspects of chemical safety management in Australian workplaces. He concluded that management of hazardous materials remains at a low standard in Australia.

Hazardous materials management is arguably the most complex element to master under the OHS umbrella. Its importance has been well recognised by the industrial partners responsible for tripartite development of OHS policy and legislation in Australia. For example, the ACTU's Butcher and Pennicuik (1999, p. 132) have pointed out that "the problem of chemicals at work has been a priority for the Australian union movement for years."

The topic of chemicals at work is serviced by numerous National and State regulations, codes of practice and guidance notes. Some of these apply generally to workplace materials and address life cycle management issues associated with manufacture, transport, storage, use and disposal of substances, while others apply to more specific situations. The National Occupational Health anid Safety Commission (NOHSC) declared a hazardous substances regulatory framework in 1993. This was developed as a blueprint for specific workplace chemical legislation in the various Commonwealth, State and Territory jurisdictions in Australia (Holland \& McEwan, 1999, p. 127). The Commission, through its Worksafe Australia arm, has produced a multitude of national standards, model regulations, 
codes of practice and guidance notes. These deal with subjects as diverse as the control of workplace hazardous substances, classification of hazardous substances, control of major hazard facilities, labelling, commercial confidentiality of data relating to workplace substances, completion of material safety data sheets and application of exposure standards (National Occupational Health and Safety Commission, 1991a,b, 1994a-f). But it goes further than this. Workplace carcinogens are dealt with separately (National Occupational Health and Safety Commission, 1995b) and there has been an attempt to address the needs of specific industry sectors, with publications such as that dealing with control of workplace substances in the retail sector (National Occupational Health and Safety Commission, $1994 \mathrm{~g}$ ). The explosives and dangerous goods area has a public saiety component and is usually covered by separate legislation and codes. A raft of guidance material, at both a national and state level, supports it. Similarly, legislation dealing with radioactive substances is usually developed and administered separately to that which applies to hazardous materials.

Chemicals have potential to impact on the workforce, on the public and on the environment. At a State level, in Western Australia, several government departments have statutory responsibilities in regard to stewardship of hazardous materials and in the responses that may be necessary when there is an emergency situation.

Thus, the hazardous materials area is both diverse and complex. If study materials are tested and are effective in such a challenging area, then they should be more so in other less complex areas of OHS.

This study has focused on approaches that apply to hazardous substances in the workplace and has dealt with materials in general, rather than with specific chemicals or groups of chemicals. It has attempted to simplify and demystify some aspects of the complex world of hazardous materials.

The Western Australian Mining Industry was chosen as a focal point for this study, partly because of proximity and access opportunities for the 
researcher, but also because the Industry has a strong commitment to OHS and is receptive to new approaches and improvement opportunities. Industry-wide collaborative effort is seen at forums provided by the Chamber of Minerals and Energy of Western Australia and within the State's statutory, tripartite structures that produce legislation, policy and guidance on OHS. Significantly, though, there has been little benchmarking activity in OHS. What there is tends to be ad-hoc, informal, usually one-on-one, and thereby limited in scope and opportunity.

The present research represents the first significant effort in Western Australia to engage a number of organisations in a systematic process to identify, evaluate and share best practice in OHS.

Benchmarking practices around the world vary from simple, informal site visits (often little more than a tour, supplemented by a few unstructured questions) to the very complex and formal variety, involving months or years of preparation, teams of people and extensive socumentation (N.W.H. Ormonde, personal communication, September 17, 1999). Unfortunately there appears to be very little between these two extremes. In the first instance, potentiaily transferable ideas or innovations may be overlooked because the approach is too cursory and is not systematic. In the second, the complexity tends to confine the approach to very big, wellresourced organisations.

Benchmarking is widely regarded as an important process in bridging the gap that separates an organisation from best practice, whether that be best in the world, best in the nation or state, best in the industry, best in the locality or, even, best in the street. However, there appears to be a paucity of materials to assist with evaluating the potential for a useful exchange between the benchmarking partners. There is little to assist with identifying and focusing on aspects that offer the most potential for mutual benefit, to help with capturing of key findings and to assist with the transfer of these into the strictures and processes of the visiting organisation. Here, the innovation or best practice item of interest may 
take the form of a product, service, tool, resource, system, concept or an approach.

There is a need to develop materials that accommodate the three elements that impact on OHS outcomes, namely, organisation of work, the workplace environment and the people factor. Furthermore, these materials need to be flexible, simple to apply but of sufficient depth and effectiveness to make the time and effort worthwhile.

This work has sought to address the issues mentioned above and to open up the benchmarking process to a much wider array of businesses. Study materials will assist with the selection of benchmarking partners and will help to identify pockets of excellence for focused attention.

This work has sought to make an important contribution to the health and safety field, and thereby to the lives of many in the workplace, by producing new tools that may be applied in practical settings and which will facilitate the smooth transfer of OHS-related technology between cooperating organisations.

\subsection{Scope of the study}

This study has dealt with hazardous materials generally and has not sought to address specific substances, groups of substances, or processes associated with the generation of particular hazards. Examples of these are lead, asbestos products and spray painting - all of which are covered by specific statutory controls.

The study has a clear occupational focus and has not sought to address environmental or community issues and impacts. It has not dealt with the explosives and dangerous goods area. Nor has it dealt with radioactive materials. Furthermore, it has been focused on workplaces under the control of the Department of Industry and Resources of Western Australia. 


\subsection{Purpose of the study}

This study has been designed with five objectives in mind:

- To develop effective tools and processes for the benchmarking of hazardous materials management practices at a medium size enterprise level (defined as having 50-200 employees) and at a large enterprise level (defined as having more than 200 employees).

- To test these products with a selection of medium- and large-sized facilities within Westem Australia.

- To review and comment on results obtained from this benchmarking activity.

- To gauge the likely effectiveness of these tools and processes as agents of change and as aids in the drive towards excellence in the management of hazardous materials.

- To assess the transferability of the tools and processes to other critical elements of OHS management.

\section{$1.5 \quad$ Research questions}

is there a suite of practical benchmarking tools and methodologies capable of the following attributes:

- Accounting for the organisation of work factor, the workplace environment factor and the people factor - as they relate to the management of hazardous materials?

- Application to both medium and large organisations?

- Application to other elements under the OHS umbrella, that is, to areas other than chemical safety?

- Identifying pockets of excellence?

- Facilitating the transfer of best practice in the management of hazardous materials?

\subsection{Operational definitions}

Accident: Includes any undesired circumstances that give rise to ill health or injury, damage to property, plant, products or the environment; 
production losses, or increased liabilities (Health and Safety Executive, 1992, p. 66).

Archetype: is the underiying structure or patterns of a particular culture. It exists in the subconscious and is imprinted in the mind at an early age (Telecom, 1994, p.3).

Audit: A systematic examination against defined criteria to determine whether activities and related results conform to planned arrangements and whether these arrangements are implemented effectively and are suitable to achieve the organisation's policy and objectives (Standards Australia, 1997, p. 6)

Benchmarking: Is a powerful organisational change practice which can strengthen all aspects of a business. It is an activity that enables organisations to determine the gap that separates them from world-class performers and assists them to develop and implement innovative improvements to their own processes. It can be used to introduce best practice into enterprises. Its power lies in its objectivity (National Industry Extension Service, 1993, p. 4).

Best Practice: The cooperative way in which firms and their employees undertake business activities in all key processes - leadership, planning, people, customers, suppliers, community relations, production and supply of products and services, and the use of benchmarking (Australian Manufacturing Council, 1994, p. 1).

Consequence: The outcome of an event expressed qualitatively or quantitatively, being a loss, injury, cisadvantage or gain (Standards Australia, 1999, p. 2).

Critical Behaviours: These are behaviours that are critical to safety. When performed safely, critical behaviours prevent injury. When performed in an at-risk manner, these behaviours constitute exposure to injury (Krause, 1997, p. 23).

Critical Success Factors: These are those quantifiable, measurable and auditable indicators of process performance in a key business process (Watson, 1992, p. 19).

Culture: This can be regarded as the integrated systems of beliefs, values, paradigms, structures, processes, language and symbols that 
influence the behaviour of groups of people. It is unique to a given group of people and newcomers normally have to leam the rules of that culture to be accepted. It begins to be imprinted from the moment of birth, operating below the level of conscious awareness (Irwin, 1994, p.3).

Enabler: A system, method, document, training or technique that facilitates the successful implementation of the particular process (Watson, 1992, p. 82).

Event: An incident or situation that occurs in a particular place during a particular interval of time (Standards Australia, 1999, p. 2).

Exposure Standard: Means an airborne concentration of a particular substance in the worker's breathing zone, exposure to which, according to current knowledge, should not cause adverse health effects nor undue discomfort to nearly all workers (National Occupational Health and Safety Commission, 1991c, p. 32).

Hazard: A source of potential harm or a situation with a potential to cause loss (Standards Australia, 1999, p. 2).

Hazardous Substance: Refers to a substance that has the potential through being used at work to harm the health or safety of persons in the workplace (National Occupational Health and Safety Commission, 1991c, p. 32).

Health Surveillance: Refers to the monitoring of individuals for the purpose of identifying changes in health status due to occupational exposure to a hazard. It includes biological monitoring (Standards Australia, 1997, p. 7).

Incident: Any unplanned event resulting in, or having a potential for, injury, ill-health, damage or other loss (Standards Australia, 1997, p. 7).

industrial Hygiene: Has been defined as that science and art devoted to the anticipation, recognition, evaluation and control of those environmental factors or stresses arising in or from the workplace, which may cause sickness, impaired health and well-being, or significant discomfort among workers or among the citizens of the community (Plog \& Olishifski, 1988, p. 3). 
Leadership: Defines what the future should look like, aligns people with that vision, and inspires them to make it happen, despite the obstacles (Kotter, 1996, p. 25).

Likelihood: Used as a qualitative description of probability or frequency (Standards Australia, 1999, p. 2).

Management: Is a set of processes that can keep a complicated system of people and technology running smoothly. important aspects include planning, budgeting, organising, staffing, controlling, and problem solving (Kotter, 1996, p. 25).

Material Safety Data Sheets: Provide information needed to facilitate the safe handling of chemicals in the working environment. MSDSs describe the properties and uses of chemical products or formulations, health hazard information, precautions for use and safe handling information (National Occupational Health and Safety Commission, 1994e, p.7).

Mixture: Is a physical combination of chemicals resulting from the deliberate mixing of those chemicals or from a chemical reaction (National Health and Safety Commission, 1999, p. 10).

Occupational Health: is the multi-disciplinary approach to the prevention of occupational disease or unacceptable discomfort. This is achieved by limiting the dose of toxic, fibrogenic or physical insult received by the worker to that below which adverse consequences are most unlikely (King, 1990, p. 315).

Performance Indicators: Are used to monitor performance of individuals, groups or whole enterprises (Kaplan \& Norton, cited in Sweeney, 1994, p. 37).

Quality Management: Is the management philosophy and management practices that aim to harness the human and material resources of an organisation in the most effective way to achieve the objectives of the organisation. (BS 7850, 1992, cited in Deacon, 1994, p.19).

Risk: is the potential for realisation of unwanted negative consequences or events. It relates to the likelihood or probability that the substance will cause harm in the particular circumstances of use at the workplace. This will depend on the hazard presented by the substance, together with 
exposure patterns, work practices, control measures applied and other factors (Malachowski, 1995, p. 235).

Risk Assessment: The overall process of risk analysis and risk evaluation (Standards Australia, 1999, p. 3).

Risk Control: That part of risk management that involves the implementation of policies, standards, procedures and physical changes to eliminate or minimise adverse effects (Standards Australia, 1999, p. 3).

Rlsk Identification: The process of determining what can happen, why and how (Standards Australia, 1999, p. 4).

Risk Management: The culture, processes and structures that are directed towards effective management of potential opportunities and adverse effects (Standards Australia, 1999, p. 4).

Risk Management Process: The systematic application of management policies, procedures and practices to the task of establishing the context, identifying, analysing, evaluating, tracking, monitoring and communicating risk (Standards Australia, 1999, p. 4).

Stakehoiders: Are those people and organisations who may affect, or be affected by, or perceive themselves to be affected by, a decision or activity (Standards Australia, 1999, p. 4).

Substance: Is any natural or artificial entity, composite material, mixture or formulation, other than an article (National Occupational Health and Safety Commission, 1999, p. 12).

Toxicity: Is the intrinsic capacity of a chemical to cause harmful effects to humans and other living organisms. It is an inherent property of a material, as is the case with boiling point, flash point and similar. Damage may be permanent or transient (National Occupational Health and Safety Commission, 1999, p. 12).

Toxicology: Can be defined as the study of adverse and potentially adverse effects of chemicals that have, or may have, the capacity to cause injury to living organisms (Malachowski, 1995, p. 237).

\subsection{Sources of information}

There is a vast body of information that deals with various aspects of chemical safety and associated management systems, tools and 
processes. For the present work, it was necessary to consult books, journals, regulations, codes, guidelines, communications, training packages and presentation materials, work colleagues and to access reliable sources via the Worldwide Web.

With regard to the Internet, this is increasingly seen as the means to deliver the latest information on topics such as OHS legislation, hazard alerts, engineering controls, safety clothing and equipment, and similar, to employers and employees (Vecchio-Sadus, 2000, p. 15). The same author identifies a number of web sites relevant to OHS in the Australian context. Davison $(2000$, p. 15) provides an international perspective on the best health and safety directories and search engines.

The references that have been used for this research are listed later in the thesis. The referencing format that has been adopted in based on the Publication Manual of the American Psychological Association (5th ed. 2001) and is consistent with guidelines published by Edith Cowan University (Jongeling, 2003).

Further guidance with respect to presentation of this work was obtained from Anderson and Poole (1998), Isaac and Michael (1981), Madsen (1992) and Edith Cowan University (2003).

\section{$1.8 \quad$ Document structure}

This document is laid out in a format and style consistent with that advocated by Edith Cowan University (2003).

Chapter 2 that follows provides a review of pertinent literature. It contains a description of modern management systems and approaches as they relate to OHS, together with sections on legal perspectives, hazardous materials management and the search for best practice.

Chapter 3 covers study design and methodological matters, including data collection and analysis, reliability and validity considerations, study deliverables to participating sites and limitations. 
Results are presented in Chapter 4. Twenty-two sub-headings are applied to each of the eight facilities that participated in the study and these are explored in tum. In addition, a section of the chapter deals with the exercise to test the transferability of study tools and processes to $\mathrm{OHS}$ elements other than chemical safety.

Chapter 5 features a discussion on the research methodologies and tools, and how these were developed and applied. Further, it covers intra-site and inter-site observations and comparisons, and relates these to the research questions.

Chapter 6 contains conclusions and recommendations. It provides an account of study outcomes, novel aspects of the work and ideas for how findings may be developed and/or applied elsewhere.

Finally, there is a reference section, followed by a set of appendices that provide detail in support of matters covered in the main body of the document. 


\section{CHAPTER 2 REVIEW OF THE LITERATURE}

\subsection{The Quality Management approach}

\subsubsection{Background, concepts and development}

Konosuke Matsushita, president of the Matsushita Electric Industrial Company of Japan, in his book Why the West will Lose stated in 1979 that:

We are going to win and the Industrial West is going to lose out. There's nothing much you can do about it, because the reasons for your failure are within yourselves (cited in Vincoli, 1991, p. 28).

At that time, Japan had embraced the principles of Total Quality Management (TQM) and as a result was exerting severe competitive pressure on other industrlalised countries of the world.

But the quality management story began some three decades earlier. In the aftermath of World War II the U.S. Government sent Dr. W. Edwards Deming to Japan to assist in the economic redevelopment of that country (Deacon, 1994, p. 20). The Japanese were very receptive to Deming's pioneering ideas and philosophy concerning total quality control (described in Deming, 1986). His concepts were adopted in Japan decades before these were taken seriously by the West (Motzko, 1989, p. 17). Other quality pioneers were Joseph Juran, Armand Feigenbaum, Phillip Crosby and Kaoru Ishikawa. All of these people presented a common message - that quality should be sought throughout the life cycle of the product, and that there should be strong emphasis on continuous improvement of processes and systems based on performance measures, minimising variation and customer feedback (Vincoli, 1991, p. 28; Sarazen, 1991, p. 34).

Mitchell (1993a, p.4) notes that the Western notion of improvement is to point out faults, defects or weaknesses and then set about putting them 
right. On the other hand, the Japanese approach is based on continuous improvement and "this is perfect - so now, let us make it better".

There are numerous definitions of total quality management. One, by the US Department of Defense (cited in Savage, 1991, p. 100), expresses it as "a focused management philosophy for providing the leadership, training and motivation to continuously improve an organisation's management and operations."

The U.S. Quality and Productivity Association (cited in Vincoli, 1991, p. 28) defines Total Quality Management as "a customer-focused, strategic and systematic approach to continuous performance improvement".

Cook and Blaxter (1991,p. 151) see the quality-aligned organisation of the future as using advanced statistical techniques to optimise human and production processes. They see people being regarded as unique and precious resources and the organisation having a clear set of values, derived from the vision, to guide behaviour. Such organisations will have a strong customer focus, with business processes managed across functional boundaries and improvement themes aimed at achieving breakthroughs in customer satisfaction. Strong partnerships will be forged with a small number of suppliers. Regular audits will be used to ensure that the organisation's vision, planning, actions and performance measures are integrated vertically and horizontally.

Successful organisations make full use of the creative forces within thern (Cane, 1995, p. 20). This means giving employees control of thei- work environment, as well as responsibility to work individually and in teams to continuously improve their work (Feigenbaum, 1991, p. 17, Roberts, 1996, p. 13). According to Pardy (1991, p. 13), "employers who are not tapping into the brain power of their employees are losing out on a most valuable source of information and knowledge". Adams (1991, p. 22) put it that "workers work in the process; managers work on the process to improve it with the workers' help." According to Aune (1991, p. 34), "a company reaping the full benefits of TQM has to make problem solvers out of as many employees as possible." Participative involvement is based on the 
belief that members of today's workforce are more highly educated and trained than ever before, that ownership and acceptance of change is higher when those affected have been part of the decision-making process, and that it takes the combined talents of all stakeholders for the organisation to excel (Aune, 1991, p. 36). Blewett and Shaw (1995a, p.17) have observed that empowered people with supportive and visionary managers rise above the mediocre, learn from errors and turn problems into opportunities. In the words of Allaire (1991, p. 66), "you need confident, empowered employees, who for the most part are working together in a collaborative effort, sparking each other's creativity in small groups and calling upon their diverse backgrounds and experiences".

Quality concepts demand a totality of commitment from the top level of management down to the workshop floor (Gerhardsson, 1998, p. 6).

The British Standard dealing with quality management, BS 7850 , refers to the attainment of maximum effectiveness and efficiency within an organisation by putting in place processes and systems which will ensure that every aspect of its activity is aligned to satisfy customer needs and all objectives, without waste of effort, and using the full potential of every person in the organisation (British Standards Institution, 1992, cited in Deacon, 1994, p.19).

Watson (1992, p. 82) points out that "Improvement processes may result in continuous incremental gains (kaizen) or lead to strategic breakthroughs that leapfrog the competition (hoshin). Many of the breakthrough improvements come from changing the business paradigm or from significant technological developments."

Robson (1991, p. 35) summed up the TQM phenomenon with the following words: "The biggest problem with total quality management (TQM) is its obviousness. To say that it is cheaper and better to do things right the first time rather than redo work, fix the problem, or simply scrap it, is so paralyzingly obvious that it masks the true nature of the changes that TQM demands." 
In the USA, the quality revolution has been encouraged and heavily promoted through the Malcolm Baldridge National Quality Awards. The equivalent in Australia is the Australian Quality Awards (National Industry Extension Service, 1993, p. 3). Occupational health and safety is embedded into the key area "people" in a section called "well-being and satisfaction" (Blewett \& Shaw, 1996c, p. 483).

\subsubsection{Culture and change}

Notwithstanding the obvious logic of the quality management approach, implementation in countries outside of Japan has met with mixed success, particularly in the early days of its introduction. In the mid-1980s, the AT\&T Corporation in the United States conducted research that established that where fundamental cultural beliefs are at odds with the quality approach being introduced then there would be strong resistance to change. They established an American archetype for quality. As indicated in the Operational Definitions section, archetypes are the underlying structure or patterns of a particular culture and tend to be imprinted into the sub-conscience at an early age. They are deeply embedded and difficult to change. However, understanding them, recognising them and responding to them will greatly assist the change management process (Merry, 1998, p. 15).

The AT\&T research led to the development of a framework that facilitated the successful deployment of quality programs within the American context. In Australia, Telecom was striving to introduce quality management and continuous improvement approaches into its operations and was aware of the AT\&T work. It decided to commission a similar study - in this case to try to understand Australian cultural characteristics and to establish how early imprinting experiences shape subsequent attitudes and behaviour. Results from the Telecom study were made available to the Australian Quality Council for further development and deployment. One of the key findings of the Telecom study was that "quality for Australians is perceived primarily in terms of the relationships people have with those around them and the organisations with which they 
are involved" (Irwin, 1984, p.5). Recognition and identity are important ingredients in a good quality relationship.

Pidgeon (1991, p. 134) advances a "working definition of culture" as "the collection of beliefs, noms, roles and practices shared within a given social grouping or population". Blewett and Shaw (1996b, p. 187) relate it to OHS with their definition: "Occupational health and safety culture, then, is the system of shared values and beliefs about OHS which create behavioural norms which guide OHS activities in the enterprise." Merry (1998, p. 15) notes that culture is difficult to evaluate because many facets are not directly observable and may even reside at the unconscious level.

There are many dimensions to culture. Apart from the characteristics that may be ascribed to society as a whole, there are the features that can be applied to sub-sets, such as industry groupings, or even, individual organisations. Watson (1992, p. 95) points out that each company has a unique culture as determined by its management style and historical perspective. Adams and Adams (1995, p. 8) note that cultures develop through the collective experiences and learning of members and, further, that all corporate cultures share common traits while still being unique.

Thus, when change is being contemplated, it is important to understand and accommodate, where possible, the cultural characteristicis that will impact heavily on successful implementation of the change. According to Brandt (1997, p. 25), "managing change is essential because it can transform the beliefs, values, attitudes and behaviours of an organisation and its infrastructure". Alpander and Lee (1995, p. 4) note that successful organisations view change not as a one-time event but as an on-going process necessary to ensure customer needs are met. They suggest that an organisation can build a climate that fosters creativity, harmony and teamwork, where continuous improvement becomes a way of life.

\subsubsection{Tools and processes}

McConnell (1989) has provided detailed guidance on the seven tools of the quality approach. Some are nothing more than ways to direct and 
improve thinking processes, while others are methods to enhance data interpretation (Adams, 1991, p. 27). In brief, these tools are:

- Flow charts

Process analysis is the first step in quality improvement. Flow charts are one of the simple, but powerful, statistical tools of variation invoived in the Deming approach (Adams, 1991, p. 27). They help analyse and understand a process. Certain conventional symbols are used when drawing a flow chart - the principal ones are rectangles (representing process steps), diamonds (representing decision points), circles (indicating that the process is continued elsewhere on the page) and arrows (indicating the direction of flow). (National Occupational Health and Safety Commission, 1996, p. 25)

- Cause and effect (CE) diagrams

These are also known as Fishbone or Ishikawa Diagrams. They were developed by Kaoru Ishikawa in 1943 and have become one of the standard tools of the quality practitioner. The "fishbone" shape of a CE diagram is a visually effective way of breaking a problem down into manageable elements (National Occupational Health and Safety Commission, 1996, p. 26; Watson, 1992, p. 80). They are used to group root causes of a problem under suitable headings (such as materials, methods, environment, people) and then to relate these to the effect (or, in some cases, the desired outcome). This then provides a basis for action. Cause and effect diagrams represent a powerful aid in creating and guiding effective problem-solving teams (Adams, 1991, p. 27).

- Pareto charts

Many years ago Vilfredo Pareto noted that $80 \%$ of wealth was held by $20 \%$ of the population. Since then, the Pareto Principle, often called "the 80-20 rule", has been applied in many areas, including business. Pareto charts are useful quality tools in that they help to separate the "vital few" from the "trivial many" (McConnell, 1989, p. 80; Adams, 1991, p. 27). Put in OHS terms, the Pareto principle suggests that $80 \%$ of the improvement can be gained through addressing only $20 \%$ of the at-risk activities, so long as the responsible activities can be identified (Sweeney, 1992, p. 92). Expressed in a different way, Pareto indicates that $80 \%$ of incidents hold 
little promise for OHS improvement and that scaling devices are needed (Adams, 1991, p. 27).

- Histograms

The histogram measures and displays how frequently something occurs. It is associated with the familiar bell-shaped curve (Adams, 1991, p. 27).

- Run charts and graphs

These display trends and are useful in monitoring anything that is long range.

- Control charts (average and range)

These monitor the process for stability.

- Scattergrams

These determine whether any cause and effect relationship occurs between two variables (Munro, 1991, p. 76).

The quality tools mentioned above generate the data needed to rark problems objectively by order of importance, to assess the suitability of improvement ideas, and to elicit broad-based agreement on the organisation's plans, priorities and resource allocation (Adams, 1991, p. 27; Sytsma \& Manley, 1999, p. 1).

Aune (1991, p. 36) lists the above items and several others, grouped under the headings of descriptive tools, creative tools, problem analysis tools and statistical tools, as devices to assist the problem solving process. He also asserts that work unit analysis (or departmental task analysis) and business process analysis (or process ownership analysis) are important techniques to be applied to problem solving. On the latter point, business processes are defined by Aune as "a group of related tasks that utilise the resources of the business to produce specified results" and "a repeatable sequence of activities that has measurable input(s), value-added activities and measurable output(s)" (Aune, 1991, p. 36). The management of hazardous substances within an organisation would involve processes that meet this definition. 


\subsubsection{Impact on management systems}

Pope (1992, p. 4) asserts that: "quality in management depends on ability to get things done by, with, and through others without operational mishaps."

John Sprouster, the CEO of the Australian Quality Council, observed in 1994 that over 15,000 organisations in Australia were actively pursuing some or all of the quality principles and practices, and furthermore, that $60 \%$ of the nation's top 55 companies were actively implementing quality concepts (Sprouster, 1994, p. 30). In the same article, he noted that "when linked together into a powerful totality, quality can be expected to lead to world class outcomes in Australian companies."

Australian organisations are required to compete more strongly than ever before - due mainly to globalisation of markets, rapidly changing customer tastes, the pace and nature of technological change and changes in labour market conditions (National Industry Extension Service, 1993, p. 1). Building a sustainable business means achieving or improving on the performance of the best, leading edge international performers. As the National Industry Extension Service (1993, p. 1) asserts, "the achievement of international best practice is no longer an option, but a necessity."

McConnell noted more than a decade ago that a pleasing feature in Australia was that the quality approach was being adopted in the public sector as well as the private sector (McConnell, 1989, p. 2).

The Australian Business Excellence Awards, formenty known as the Australian Quality Awards, are based on seven categories of performance - leadership, policy and planning, information and analysis, people, customer focus, quality of process, product and service, and organisational outcomes (Business Excellence Australia, 2003, p. 1).

The processes, principles and standards used for these awards have been adapted and adopted for use within the Australian Mining Industry. Since 1994, the Minerals Council of Australia has provided the National Minerals 
Industry Excellence Awards for Health and Safety (the MINEX Awards). The objectives of the Awards are to:

- Reward best practice, excellence and/or improvement based on comparisons of performance and practices.

- Provide peer assessment of OHS management systems against Awards criteria.

- Provide benchmark information for self-assessment of OHS management systems by mining and minerals processing companies.

- Promote the industry's commitment to improved OHS performance (Minerals Council of Australia, 1997, p.2).

The evaluation process is built around an examination of six key elements of an OHS management system, namely leadership, health and safety management, health and safety processes, people, information and analysis, and performance (Minerals Council of Australia, 1997, p. 4). There is recognition that different organisations will have different cultures, technologies and operating environments and may choose to achieve the same outcomes in different ways. A five-point model, referred to as the IADRI model, establishes a framework for the assessment. The acronym, IADRI, covers intent (the purpose and expected outcomes), approach, deployment, results and improvement (how changes were made from lessons learnt). A scoring system is used with points allocated to each of the six elements mentioned above (Minerals Council of Australia, 1997, p. 7).

\subsubsection{Impact on OHS}

Numerous authors have noted that the terminology, concepts and approaches described in the quality management literature can be readily applied in relation to the management of OHS (Strobach, 1990, p. 42; Fisher, 1991, p. 27; Mitchell, 1993a, p. 3; Famell, 1991, p. 39; Saunders, 1995, p. 585; Arnis and Booth, 1992, p. 46; Health and Safety Executive, 1992, p. 12; Deacon, 1994, p. 19; Worksafe Australia, 1995, p. 13).

According to Fisher and Oxenburgh, the factors that lead to operational mishaps and accidents are often the same underlying factors that 
influence productivity, quality and efficiency. Thus, if the causes of operational mishaps are identified and corrective action is applied, there should be an improvement in quality and productivity (Fisher, 1991, p. 23; Oxenburgh, 1991). This is a view supported by the United Kingdom Health and Safety Executive (1992, p. 12). That body points out that the ultimate goal in health and safety is an injury-free environment. Furthermore, organisations pursuing this goal are not normally acting out of purely philanthropic motives. "They have clearly recognised that accidents and ill-health cost money and that an effective system for managing heaith and safety will help reduce what in quality terms is known as the cost of non-conformance" (Health and Safety Executive, 1992, p. 12).

One of the key quality objectives is elimination of waste. Tye and Brown (1990, p. 24) point out that "one of the major waste-causing agents is accidents and the lack of any type of damage control results in damage to equipment, property and in worker disablement and death".

In the words of Famell (1991, p. 40), "Companies which are able consistently to provide high levels of safety performance have made the connection between total quality management and safety". Furthermore, he said, "It is only when organisations have achieved high levels of quality that the on-going safety performance can consistently be maintained and risk minimised" (Farnell, 1991, p. 44).

Sometimes, this is seen in reverse. Pardy $(1991, \mathrm{p} .12)$ observes that "by focusing on safety, Alcoa was able to unlock and access the whole tool chest associated with total quality management."

Deacon (1994, p. 19) comments that "the fact that health and safety is now seen as an integral part of TQM is surely of immense significance and marks a watershed in the historical development of health and safety within the UK!"

Deming emphasised that it is the system of work that determines how work is performed, while Mitchell points out that only managers are in a 
position to allocate resources, provide training, select the equipment and tools to be used, and provide the plant and environment needed to achieve high standards of OHS. The workforce is more likely to be involved in resolving special OHS problems caused by actions or events directly under their control (Mitchell, 1993a, p. 3). Continuous improvement is the central concept reflected in the Deming/Shewhart Plan-Do-Check-Act cycle and is a key concept in the ISO-based Occupational Health and Safety Management Systems (OHSMSs) that are now being deployed in many parts of the world (Redinger \& Levine, 1998, p. 579).

Strobach (1990, p. 42) notes that "as with quality, improvement (in OHS) is a never-ending cycle which requires the support and participation of the individual employee, supervisor and most important of all, top management."

Worksafe Australia (1995, p. 9) calls for integration of health and safety into broader management systems - to improve work environments and to prevent the emergence of new hazards. Integration is of assistance in avoiding conflict or confusion between operational demands and OHS needs.

In a similar vein, Amis and Booth (1992, p. 43) comment that: "management should manage safety in just the same way as other company functions, such as production, quality and finance, are managed. Good management involves a process of goal and standard-setting, and monitoring to determine whether targets are achieved."

Mitchell (1993b, p. 2) is a strong advocate of the link between quality and safety. His opinion is that "the greatest opportunity for improving safety performance lies in improving the quality of the management system." He identifies 12 principles that fit with the views Deming, Juran, Crosby and other leaders of the Quality movement. These are:

- Creating a constancy of purpose.

- Getting commitment to improved OHS that runs from the top of the organisation to the bottom. 
- Emphasising a long-term perspective.

- Benchmarking to improve key processes impacting on success.

- Focusing on continuous improvement of the OHS management processes.

- Assigning ownership for operation and improvement of the OHS process to line management.

- Accurately measuring improvements.

- Fostering a cross-sectional team approach.

- Establishing an error-free performance standard.

- Educating, training and re-training in these areas.

- Recognising that the costs of imperfection (operational errors), if corrected, have an immediate effect on the bottom line performance, customer service and customer relations.

- Getting it right first time (prevention, not inspection).

(Mitchell, 1993c, p. 13)

\section{$2.2 \quad$ Legal perspectives}

\subsubsection{Background}

State intervention in the OHS area dates from the period of the Industrial Revolution in Britain - when working conditions gave rise to a high incidence of injury and ill-health. For more than a century the British approach was a highly prescriptive one and a whole body of complex regulations developed (Deacon, 1994, p. 18).

Bottomley $(1994$, p. 2) has noted that an obvious limitation of this approach, when applied in isolation from other tools, is the focus on minimum standards rather than best practice in workplaces. Amis and Booth (1992, p. 44) comment that "the character of traditional prescriptive law may encourage a perception that safety can be 'managed' by rote compliance with specific legislation."

A watershed with respect to legislative approaches to OHS occurred in Britain in 1970 with the setting up of a committee of inquiry under the chairmanship of Lord Alfred Robens. Its task was to assess the 
shortcomings of existing health and safety legislation, namely its fragmented, inflexible and out-of-date character, and to propose more effective means of achieving desirable OHS outcomes. Its report, known as the Robens' Report, was presented to the British parliament in 1972 and gave rise to the Health and Safety at Work Act of 1974. Recommendations were built around two key elements: firstly, that legislation should embody the common law principle of duty of care and, secondly, that there should be provisions to ensure that employees are given the opportunity to input into the development and implementation of solutions to local safety problems (Laing, 2002, p. 10).

Robens (cited in Laing, 2002, p. 10) argued that "the primary responsibility for doing something about the present levels of occupational accidents and disease lies with those who create the risks and those who work with them". He advocated a flexible system under which "employers and workers would consilt and achieve a high degree of 'self-regulation', supported by general iegislative requirements and voluntary codes and standards" (cited in Laing, 2002, p. 11).

The Robens Report and its legislative outcome had a wide impact on the industrialised world and many countries have now introduced legislation along similar lines. In 1981, the Robens' principles were incorporated into ILO Convention No. 155 and Recommendation No. 164. Convention 155 set national policy standards for $\mathrm{OHS}$ for ratifying member companies. It establishes duties and obligations in areas such as workplace cooperation, provision of information and training, and duties of designers, manufacturers, importers and providers of machinery, equipment or substances for occupational use (Laing, 2002, p. 11). The Robens' principles have been incorporated into legislation in all Australian States and Territories (Laing, 2002, p. 11).

Legislative approaches cannot of themselves control the vast number of circumstances that exist in the industrial world. Winder (1995, p. 214) points out that there are many problems in relying on regulatory approaches to deal with chemical safety. For example, health outcomes 
such as dermatitis, neuropathy, cancer and other chronic diseases are not amenable to solution by regulation.

Deacon (1994, p. 21) asserts that the improvements in OHS performance should come from growth of OHS culture, rather than as a consequence of the threat of legal action.

According to Bottomley $(1994$, p. 3), "the prescriptive legislation approach does not address the OHS culture of the workplace, except perhaps in a negative way by encouraging minimum compliance and evasion of inspection/audit by the regulatory authority." He pointed out that prescriptive legislation has an insidious influence on culture through the attitudes it engenders and encourages. On the other hand, performancestyle legislation, by its nature offers encouragement for the development of innovative solutions (Bottomley, 1994, p. 3).

Robens (cited by Laing, 2002, p. 10) also had something to say on this matter. He pointed out that there are severe practical limits on the extent to which progressively better standards of OHS at work can be achieved via negative regulation by external agencies and called for a more effective self-regulating system. He demonstrated a strong preference for non-statutory forms of guidance, especially codes of practice.

\subsubsection{Development and trends - international}

The International Labour Organisation has produced two initiatives dealing with the safe use of chemicals at work - ILO Convention 170 and Recommendation 177 (International Labour Organisation, 1990). The content of these has played a part in shaping the hazardous substances legislation that has been developed in Australia (St George, Lingard \& James, 1999, p. 137). In the United Kingdom, hazardous substances are covered by the Control of Substances Hazardous to Health Regulations (COSHH). The latest version of these came into force in March 1999. $\mathrm{COSHH}$ provides a comprehensive and systematic approach to the control of hazardous substances at work. The regulations require employers to 
assess the risks to health, prevent or control exposure and, in some situations, carry out health surveiltance (Parker, 1999b, p. 1).

Another important legislative development in the United Kingdom was the Chemicals (Hazard Information and Packaging for Supply) Regulations 1999. These lay down the rules by which suppliers must classify and label hazardous chemicals. This information is helpful in carrying out $\mathrm{COSHH}$ assessments (Parker, 1999a, p. 1).

Europe has also seen a lot of recent activity in relation to legislative controls for chemical safety. In 1998, the European Union signed an intemational convention aimed at improving the regulation of trade in chemicals. The convention also contains requirements for labelling initiatives aimed at promoting the safe use of chemicals (Parker, 1998, p. 1).

In the United States, the Toxic Substances Control Act (1976) is one of the key legislative instruments being applied in the chemical safety area. There are many regulations, directives and standards of legal force that elaborate on the requirements for testing, inspection, notices, reporting, disclosure of data, employee protection, enforcement, and similar that are provided for in the principal legislation (Anon., 2003, p. 1).

\subsubsection{Development and trends - Australia}

in Australia, the tripartite National Occupational Health and Safety Commission (NOHSC) is the body responsible for leading and coordinating national efforts to prevent or reduce the incidence and severity of injury and disease. The National Commission aims to:

- Achieve best practice in OHS regulation through national coordination of development, implementation and evaluation of national OHS standards.

- Provide an effective nationan tripartite forum to address OHS issues of naticnal importance. 
- Provide the basis for targeting OHS activities by producing statistical reports using existing information systems and identifying and developing additional cost-effective data sources.

- Achieve OHS research outcomes supporting the development of national standards.

- Contribute to industry performance through independent assessment of industrial, agricultural and veterinary chemicals for their occupational health, public health and environmental effects.

(National Occupational Health and Safety Commission, 1996b, p. iii)

A set of model regulations for the control of workplace hazardous substances began to evolve in Australia in the late 1980 s. The National Commission's initial version was released in 1991, followed by a modified effort in 1994. It used classification criteria for hazardous substances developed by the European Community, the experience of Britain's Control of Substances Hazardous to Health (COSHH) regulations and the International Labour Organisation's Convention 170 (Winder, 1999a, p. 100; Holland \& McEwan, 1998, p. 128).

For several years in the early 1990s, the output of material from the National Commission with respect to chemical safety was prolific (National Occupational Health and Safety Commission, 1990, 1991a,b, 1994a-g, $1995 a, b, 1996 b, 1999)$. This material gives guidance on topics such as labelling, placarding, material safety data sheet preparation, commercial confidentiality of data, exposure standards, control and storage of hazardous substances, assessment of health risks, and many related chemical safety issues.

The process of implementation in the States and Territories has been somewhat tortuous. There were problems with overlap with existing legislation (such as that covered by poisons and dangerous goods Acts), State-Commonwealth jurisdictional issues, together with problems with classification of hazardous substances and scope of the legislation. These problems and the history of the development and deployment of the legislation throughout Australia have been summarised by Winder 
(1999a). Under the National Model Regulations for the Control of Workplace Hazardous Substances, a hazardous substance is either listed as such on the NOHSC List of Designated Hazardous Substances or has been classified as such by the manufacturer or importer in accordance with the NOHSC publication Approved Criteria for Classifying Hazardous Substances (National Occupational Health and Safety Commission, 1999, p. 2).

In Australia, risk management-based workplace hazardous substances regulations are in place in all States and Territories. These impose significant legal obligations and responsibilities on manufacturers, suppliers, employers and others in relation to the use of chemicals (Winder, 1999b, p.168).

Hazardous substances legislation based on the risk management approach features the broad elements of identification, assessment and control. According to Winder (1999b, p. 162), these can be represented as:

- Obligations for manufacturers, importers, suppliers, employers, employees and others.

- Requirements for record-keeping.

- Criteria for determining a hazardous substance.

- Requirements for hazard communication.

- Procedures for workplace assessments.

- Requirements for exposure control, including permissible exposure standards.

- Consideration of the need for workplace monitoring.

- Consideration of the need for health surveillance.

- Requirements for education and training.

- Systems for the emergency services.

Some jurisdictions have adopted a step-wise approach to implementation. For example, in NSW a model program was piloted at the State Railway Authority to provide feedback on practical issues before legislative requirements were enacted (St George, Lingard \& James, 1999, p. 138). 


\subsubsection{Development and trends - Western Australia}

In 1984, Robens-style UHS legislation was introduced into Western Australia. The objectives of the Occupational Health, Safety and Welfare Act 1984, as expressed by the Department of Occupational Health, Safety and Welfare of Western Australia (1994a, p. 3) are:

- To promote and secure the health, safety and welfare of people at work

- To protect people at work against hazards

- To assist in securing safe, hygienic work environments

- To reduce, eliminate and control hazards

- To foster co-operation and consultation between employers and employees.

The Act sets out duty of care responsibilities for employers, employees, contractors, manufacturers, designers, importers, suppliers and other parties that can potentially impact on health and safety at work. The duties established for employers fall into five principal areas. These are:

- Provide and maintain workplaces, plant and systems of work that do not expose employees to hazards.

- Provide information, instruction, training and supervision so that employees are not exposed to hazards while the! are working. The employer is only required to provide training relevait to the health and safety of employees in that workplace.

- Consult and co-operate with health and safety representatives.

- Provide adequate personal protective clothing and equipment where it is not practicable to avoid the presence of hazards.

- Make arrangements for the safe use, cleaning, maintenance, transportation and disposal of substances and plant used in the workplace.

(Department of Occupational Health, Safety and Welfare of Western Australia, 1994b, p. 5)

This legislation applies to all people in workplaces in Western Australia, except those on mine sites or in Commonwealth agencies who are covered by separate legislation. For the Mining Industry, the Mines Safety 
and inspection Act 1994 is the prevailing piece of primary legislation (Department of Minerals and Energy, 1994). Its fundamentals mirror those of the Occupational Health, Safety and Welfare Act, but put them into a mining context. The Act is supported by the Mines Safety and inspection Regulations 1995 and by numerous codes, standards and guidelines. The hierarchy of acts, regulations, codes of practice and guidelines is provided in Appendix 2. Responsibility for the enforcement of the mining legislation rests with the Department of Industry and Resources of Western Australia (formerly the Department of Minerals and Energy of Western Australia). The Act covers all aspects of mining, from exploration, through to development, production, closure and rehabilitation. Extractive industry is included, along with treatment plants, smelters, refineries, dedicated ports and rail systems. The Act has key provisions dealing with general duty obligations for employers, employees, self-employed persons, suppliers, manufacturers and importers, and consultation between the parties (Australian and New Zealand Minerals and Energy Ministerial Council, 1999, p. 28). The Regulations contain a division on hazardous materials management and include requirements for registers of material safety data sheets and hazardous substances, labelling, risk assessment, workplace monitoring and strategies to reduce risk (Department of Minerals and Energy, 1995a).

Additionally, the Department has developed a guideline to provide further detail and guidance on what is expected in the area of management of hazardous substances (Department of Minerals and Energy, 1997a).

The Regulations refer to several NOHSC Regulations and Codes of Practice, Australian Standards, and the Australian Code for the Transport of Dangerous Goods by Road and Rail. In addition, the Dangerous Goods Regulations 1992 apply to mines, where many dangerous goods also qualify as hazardous substances.

For non-mining workplaces, chemical safety is dealt with in Part 5 (Hazardous Substances) of the Occupational Safety and Health Regulations 1996. Part 5 covers a similar range of topics to that described 
earlier for mine sites in Western Australia (Worksafe Western Australia, 1996).

Chesson $(1995, p .1)$ has described the processes applied locally in the development of a hazardous substances legislative package. To a large extent, the National Model Regulations and National Code of Practice have been adopted in Western Australia.

\subsection{OHS management}

\subsubsection{Background}

Sweeney $(1992$, p. 89$)$ notes that process safety is governed by complex interactions of the following:

- Management systems

- Process technology

- Human behaviour, and

- External events.

Sirnilarly, Hurst (1998, p. 58) emphasises the strong linkages between safety management, human error, safety cultures and risk assessment.

Farnell (1991, p. 39) has identified three approaches to safety management in evidence over the last few decades. Firstly, there is the traditional approach - characterised by close surveillance of the workforce, together with correction of unsafe behaviours via the disciplinary process, signs, posters, and handout materials to remind employees to 'work safely. This was superseded by an approach described as the procedural-engineering approach. The latter was based on reliance on written procedures and training on these, together with use of engineering solutions to eliminate or neutralise accident sources. Finally, the behaviour-based approach has become popular. It features a belief that the workforce consists of mature human beings who will be motivated to work safely when there is management understanding and adoption of systems related to their psychological needs. It is built upon involvement, commitment and a focus on corporate safety goals. According to Famell (1991, p.40), "organisations which positively influence 
human behaviour to minimise errors are those producing a climate which promotes employee commitment to health and safety and which emphasises that deviation from corporate safety goals is not acceptable."

Mitchell (1993a, p. 1) emphasises the importance of visible management commitment. Senior managers alfocate resources, decide strategies for risk management, and select and implement the management processes that will enable the organisation to meet its OHS goals.

Pope (1992, p. 4) points out that "personal harm and property damage are symptoms of operational mishaps that become 'windows of opportunity' to evaluate the quality of management."

Kotter (1996, p. 128) has expressed the view that the essence of management is to systematically target objectives and budget for them, create plans to achieve these objectives, organise for implementation, and then control the process to keep it on track. These elements are clearly relevant to OHS - and to sub-sets such as the management of hazardous materials.

In similar fashion, Jenkins, Brearley and Stephens (cited in Deacon, 1994, p. 19) have pointed out that the role of management is to develop and promote an appropriate organisational culture, to formulate, communicate and implement a suitable policy, and to monitor the organisation's performance.

Leadership is a key to getting results in OHS (Juran, 1991, p.7). As the Health and Safety Executive (1992, p. 2 ) puts it, "the vision, values and beliefs of leaders become the shared 'common knowledge' of all." According to Lindsay (1992, p. 390), "the visible and active leadership of directors and senior managers develops and maintains a culture considerably supportive of health and safety management. They aim not simply to avoid accidents, but to motivate and empower people to work safely." Petersen (1997, p. 45) talks in terms of management accountability as "a system of role definition, correct measures of 
performance and adequate rewards that are contingent on that performance - forces managers to take pro-active actions every day."

The development and application of performance standards is a key element of the management function. The Health and Safety Executive (1992, p. 17) highlight the need for performance standards that:

- Set out clearly what people need to do.

- Assist in identifying the competencies that are needed.

- Form the basis for measuring individual, group and organisational performance.

Strobach (1990, p. 41) points out the difference between "leading" people and "managing" people. He comments that "if executive management wishes to make safety a way of life for their people, they should strive to make safety a way of life for themselves."

The DuPont organisation is widely acknowledged as a world leader in safety performance. It believes that all occupational injuries and illnesses can be prevented and that this is a realistic goal, not just a theoretical objective (Scott, 1999, p. 8). The company has plants with over 1,000 employees that have operated for over 10 years without a lost time injury. Accident rates across its operations are usually more than an order of magnitude better than the U.S. Chemical Manufacturers Association average (cited in Farnell, 1991, p. 41). DuPont is quoted by the Department of Minerals and Energy (1995b, p. 10) as asserting that "if you can't manage safety, you probably can't manage business ... If you can manage business, you probably can manage safety." This is a theme that other industry leaders have also expressed. According to Alcoa's former CEO and Chairman of the Board, Paul O'Neill (O'Neill, 1991, p. 52), "safety is the primary indicator of how well a company can lead globally." O'Neill has also been attributed with: "you can't get safety unless you really understand the process" (cited in Pardy, 1991, p.12). The Business Council of Australia (cited in Worksafe, 1995, p. 2) found that amongst its membership, workplace OHS rated as one of the most important factors in achieving business objectives. 
The DuPont experience, as expressed by Herbert (1994, p.5), has been that "the single, strongest correlation to actual safety performance involves employees' perceptions of management commitment." This is consistent with the findings of others. Dedobbeleer (cited in Blewett \& Shaw, 1996b, p. 189) identified two critical dimensions in regard to risk perception and its impact on the safety climate:

- Workers' perception of management commitment, and

- Workers' involvement and control, including their perceptions of the risks they encounter.

Involving employees and instilling a sense of ownership is another quality management fundamental that finds expression in the OHS area. Strobach (1990, p. 42) said: "One of the basic truths in management is that 'people support what they help create'." Thus, it is essential that the workforce is involved in the planning and implementation of the various OHS efforts being pursued by the organisation. Cook and Blaxter (1991, p. 151) highlight the importance of ensuring that everyone shares the organisation's vision. They comment that when the vision is supported by measures, objectives and a set of values, the vision may be transferred from the head to the heart.

Pardy (1991, p. 13) notes that "employee empowerment and participatory management have three basic virtues: they assume that several heads are better than one and that participation will improve the quality of decision-making; they motivate those who have had a hand in the decision-making process to carry out those decisions; and they help to develop and train employees involved in the decision-making process."

\subsubsection{Conceptual Framework}

A number of authors have noted that there appear to be three elements to be managed in the quest to achieve the elimination of accidents and illhealth at work. 
Firstly, there is the physical environment that constitutes the workplace. Bottomley (1994, p. 1) refers to this as "hardware" and cites examples of plant, equipment, substances, materials and working conditions.

Secondly, there are the organisation's management systems. Bottomley (1994, p. 1) refers to these as "software" and suggests that policies, standards, procedures, training systems, level and types of supervision, and communication systems are good examples.

Thirdly, there is the element that is related to people - attitudes, beliefs, behaviour, physical and mental ability to perform tasks, and similar. Bottomley $(1994$, p. 1) refers to this as the culture of the organisation although, given material presented later in this work, it is probably wider than that.

Alcoa (1995, p. 1-4) refers to shared accountability for an incident-free workplace and nominates similar groupings of elements - under the headings the work environment, the work and the workforce. Quinlan (1999, p. 21) refers to the mix of organisational, technical and behavioural factors that play a part.

Wyatt (1995, p. 19, 001) lists five groups of factors that should be considered in analysing systems of work. These are job demands, people, equipment, the working environment (physical and organisational) and outside factors. This arrangement of items is not convincing. For example, the "job demands" list refers to speed, accuracy, manual handling, shift work and control over work. These iterns would seem to fit betler elsewhere (under the "organisation of work" category).

Lindsay (1992, p. 396) represents these concepts with a circle for peopls factors (including behaviour, suitability and competence) overlapping with a circle for job factors (including the premises, plant, substances and procedures in use). A larger circle surrounds these - depicting organisational factors that impact on the other elements. These include relevant policies, standards, rules and procedures. 
Behavioural safety expert, Scott Geller, adopts a slightly different stance. He emphasises that unwanted outcomes are derived from environmental, behavioural and personal factors, and that these elements are "interactive, dynamic and reciprocal." He uses "environmental" to encompass the organisational environment as well as the physical environmert at the workplace (Geller, 1998, p.17).

Merry $(1998$, p. 18) focuses on safety culture and asserts that this has three interactive dimensions - safety climate (psychological dimension), safety management (organisational dimension) and safety behaviour (behavioural dimension).

Ragan (1997, p. 27) points out that incident causes may be traced to all three business processes (referred to by him as "human, work environment and organisation") and that no one element should be exclusively blamed for the unwanted event. He draws a parallel with the well-known fire triangle - a concept that decrees that a fire cannot occur unless all three elements (fuel, oxygen and a source of ignition) are present.

Thus, a useful conceptual framework may be built around considerations of the work environment, the organisation of work and people factors. This is represented in Figure 1 below. The framework appears to apply to OHS, in general, and to sub-elements such as the management of hazardous substances.

\subsubsection{OHS and organisational effectiveness}

Pope $(1992$, p. 4) points out that safety management fits within the total organisational effort needed to avoid performance flaws and to achieve corporate excellence. In similar vein, Merry (1998, p. 15) describes safety as a strategic business issue and an instrument for potential competitive advantage.

Organisational effectiveness is a critical lever in the achievement of excellent performance across a range of key result areas that have been defined for the enterprise. Safety performance is usually one such area. 
The interface of OHS and productivity, in combination with other factors, can make safety one of those volatile issues that bring out adversarial tensions in an organisation (Marcombe, Krause \& Finley, 1993, p. 15). When properly executed, occupational health and safety programs should prove highly effective for an organisation. They will contribute to profitability by reducing time lost through injury or disease, by reducing premiums, maintaining a fit and healthy workforce, contributing to morale, reducing liability concerns, providing marketing support and in many other ways (Toohey, 1987, p. 235; Oxenburgh, 1991, p. 14).
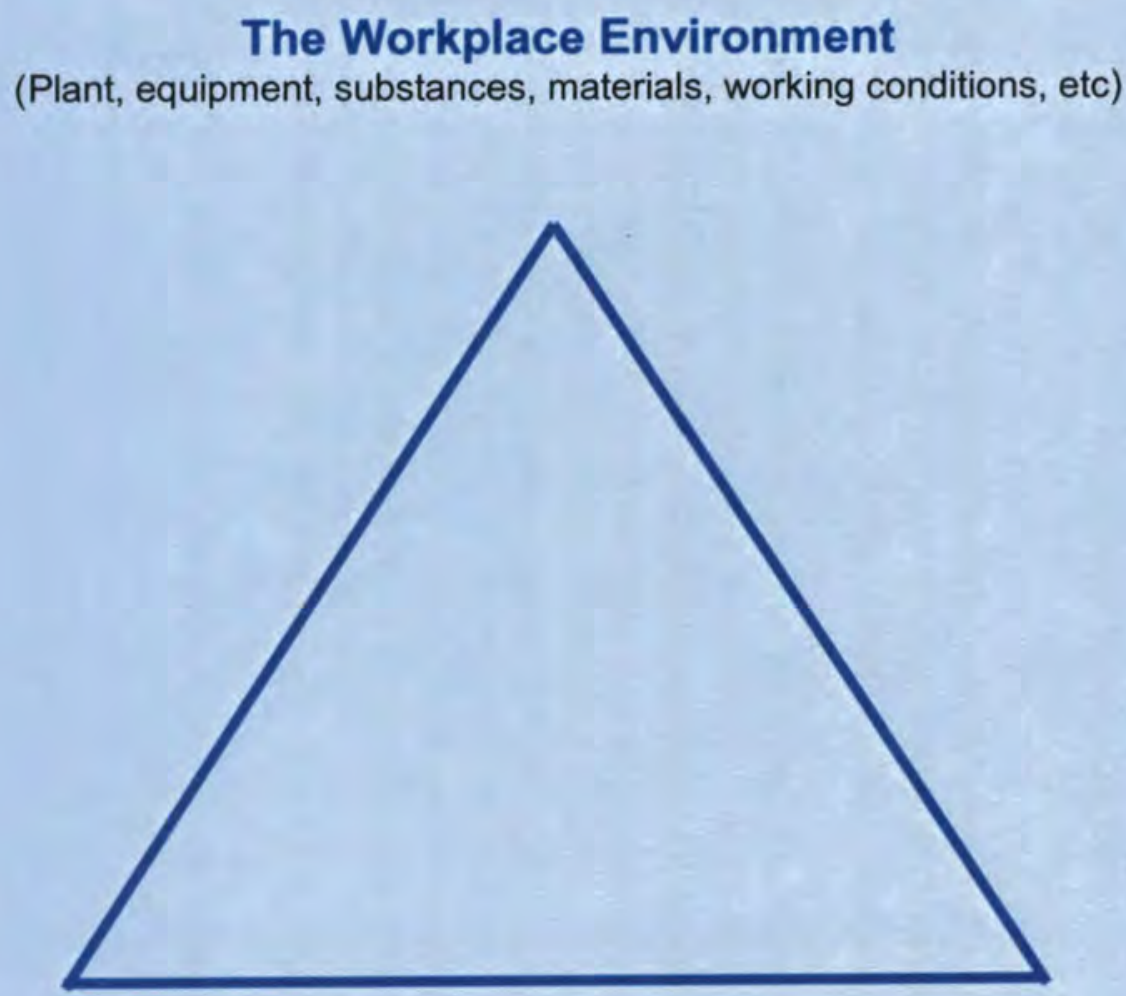

Organisation of Work (Standards, procedures, training systems, level and types of supervision, communication systems, etc)

\section{People Factors}

(Attitudes, beliefs, behaviour, physical and mental ability to do the tasks, etc)

Figure 1: A conceptual framework for $\mathrm{OHS}$ 
Wilson (1999, p.3) identifies a fit and well-motivated workforce, described as "healthy, happy and here", as a valuable asset for the organisation. Furthermore, like the no-less-valuable corporate image, the people asset is easily damaged and difficult to repair (Wilson, 1999, p. 3). Why then don't all organisations pursue OHS with great vigour and why is it that some enjoy great success while others fail quite dismally?

According to Tooney (1987, p. 237), the solution lies in a shift in thinking to recognise that workplace mishaps are a reflection of organisational effectiveness. He points out that "occupational health and safety problems are essentially rooted in the decision-making structures of the organisation and that symptoms present as clinical, technical, legal and industrial outcomes." Winder expresses a similar view. According to him, accidents, injuries and spills cannot be dismissed as aberrations. Performance in chemical safety is a reflection of the way an organisation functions (Winder, 1995, p. 213). These views are in line with those of Deming, Juran and other leaders of the Total Quality Movement, who noted that all processes exhibit variation, everyone works in a process and that emphasis should be on controlling the process, not the output (Deming, 1986; Juran, 1991).

Farnell (1991, p. 41) notes that in quality-oriented companies with excellent safety records there is "no most efficient way" or "best for quality way" or "safest way" to do the job - there is only "the right way", which incorporates all of these aspects. There is emphasis on leadership and management controls from the top, together with an emphasis on responsibility and training from the bottom upwards. A culture change has usually been achieved in such organisations, with human factors being seen as having paramount importance.

Pardy $(1991$, p. 12) advocates a six-step process to define safety in the organisation and to develop a strategy that is pragmatic, cost-effective and involves all levels in the organisation:

- Safety must be integrated into the existing fabric and corporate culture of the organisation. 
- Empower employees as part of the accident and incident problemsolving and safety performance improvement team.

- Accident and incident reporting should be encouraged, even valued, as part of the problem-solving and safety performance process.

- Tabulate, measure and fix accountability for all accident costs.

- Take what you have and make it better.

- Measure all aspects of the efforts going into the safety process.

Rimmington has addressed the issue of establishing a safety culture within an organisation. According to him, the main principles involved are:

- The acceptance of responsibility at ard from the top, exercised through a clear chain of command, seen to be actual, and felt throughout the organisation.

- A conviction that high standards are achievable through proper management.

- The setting and monitoring of relevant objectives and targets, based upon satisfactory intemal information systems.

- The systematic identification and assessment of hazards, and the deployment of preventive systems that are subject to audit and review. In such approaches, particular attention is given to the investigation of error.

- Immediate rectification of deficiencies, and

- The promotion and reward of enthusiasm and good results.

Rimmington (cited in Amis \& Booth, 1992, p. 46).

Stephan (2001) provides an overview of the determinants of safety culture within organisations and demonstrates how management improvement processes, such as total quality management, safety management plans and risk assessment processes may be applied to improve safety performance in the mining industry. He argues that the new paradigm of safety management - the socio-technical systems paradigm that considers both the engineering and human factors responsible for accidents - will significantly improve industry safety performance (Stephan, 2001, p. 237). 
Pardy $(1991$, p. 16) observes that companies with low incident rates have the following characteristics:

- Greater management involvement in safety programs, identified through managerial performance standards.

- A more humanistic approach in dealing with employees, stressing frequent positive contact and interaction.

- Better employee selection procedures.

- Use of lead workers, as opposed to supervisors, to train employees.

- Strong housekeeping and general plant cieanliness.

- Better plant environmental qualities.

- Lower turnover and absenteeism.

Toohey (1987, p. 238) proposed a model for organisational effectiveness in OHS that contains the following elements:

- A clearly expressed OHS policy

- A commitment to exceed minimum standards

- Integration of OHS procedures into the activities of the organisation

- Clearly defined performance expectations and measures for all levels within the organisation

- Injury management plans

- Management commitment and involvement

- Clearly assigned OHS responsibilities for managers and supervisors, expressed in terms of their normal duties

- Commitment and involvement of employees, with formal and informal structures to encourage their participation

- A recognition that corporate investment in OHS is good business practice.

Not everyone agrees with the thesis that good OHS performance is good for business. Wooden and Vandenheuvel studied four types of evidence (case studies, correlational research, studies on the impact of governmental regulation and general equilibrium analyses) before concluding that improved performance in safety appears to be a byproduct associated with the introduction of productivity enhancing technology, rather than a direct cause of increases in productivity 
(Wooden \& Vandenheuval, 1999, p. 415). They did go on to concede that there is a possibility that safety and productivity are the result of common factors - a view that is in line with the broad thrust of material presented in this document.

\subsubsection{Management of Change}

2500 years ago, the Greek philosopher, Heraclitus (cited in Savage, 1991, p. 100) stated: "there is nothing permanent except change." In today's world, it is necessary to do more with less. Managing change is essential because it can transform the beliefs, values, attitudes and behaviours of an organisation and its infrastructure (Brandt, 1997, p. 25).

However, change is often resisted. When an organisation undergoes significant change, this usually means changing roles, especially in middle management, and this creates insecurity and fear of losing status (Cook and Blaxter, 1991, p. 151).

Blewett and Shaw (1996a, p. 51) suggest that "organisational change comes in varied forms: it may mean anything from organisational restructuring, including downsizing, de-layering or the introduction of autonomous teams, to the introduction of new technology, hardware, software or new production machinery."

The British Standards Institute (1996, p. 13) suggests that the type of changes that might affect $\mathrm{OHS}$ include:

- Changes in staffing.

- Proposals for new product, plant, processes or services.

- Changes in work procedures.

- Process modifications.

- Software modifications.

Furthermore, changes in the external environment may be important. New legislation and developments in OHS knowledge and technology are provided as examples of this (British Standards Institute, 1996, p. 14).

Brandt (1997, p. 25) identifies seven elements associated with successful change initiatives: 
- A governing strategy that maps the course of change and an implementation plan that plots the steps by which change will occur.

- Leadership from a strong and involved management group.

- The authority and power to implement the new programs and overcome expected resistance.

- A communication plan and strategy.

- A plan for using and applying information technology.

- A management scorecard - a cycle of performance data collection, measurement, assessment and adjustment.

- A plan to change the organisation's infrastructure, including the recognition and reward system, compensation and employee accountability.

He cautions that, since organisational cultures differ, each proposed change should be tailored to meet the unique needs of each organisation (Brandt, 1997, p. 25).

Kotter, 1999, p. 21) proposes an eight-step change process that applies to successful change of any magnitude in organisations:

- Establishing a sense of urgency.

- Creating a guiding coalition (team).

- Developing a vision and strategy.

- Communicating the change vision.

- Empowering broad-based action.

- Generating short-term wins.

- Consolidating gains and producing more change.

- Anchoring new approaches in the culture.

On the last point, his view is that to successfully anchor change in the organisation, two factors are important. Firstly, there needs to be a conscious attempt to demonstrate how specific behaviours and attitudes have helped improve performance. Secondly, the next generation of managers needs time to "personify the new approach" (Kotter, 1999, p. 14).

Lewis Lehr, chief executive of the 3M Company (cited in Aune, 1991, p. $35)$, once said: "Perhaps our biggest need at $3 \mathrm{M}$ is for people who are 
uncomfortable without [emphasis added] change. Quality is the top priority and umbrella for managing change."

\subsubsection{Management Systems and Related Standards}

Blewett and Shaw (1996c, p. 484) state: "all enterprises have a management system, that is, a system which describes the ways in which inputs and activities in the enterprise are managed to produce the outputs and to position the enterprise to maximise outcomes."

Many governmental and private organisations throughout the world have developed, or are in the process of developing, Occupational Health and Safety Management Systems (OHSMSs) (Redinger, 1997, p. 32). These have been defined by Gallagher (cited in National Occupational Health and Safety Commission, 2001, p.1) as "a combination of the planning and review, the management organisational arrangements, the consultative arrangements, and the specific program elements that work together in an integrated way to improve health and safety performance".

The International Organisation for Standardisation (ISO) has played a significant guidance role in this area via the development of its quality assurance system model, ISO 9001, and its environmental management systems model, ISO 14001 (Dyjack \& Levine, 1995, p. 599; McGinley, 1996, p. 30).

In 1996, ISO considered the development of an Occupational Health and Safety Management standard but opted not to pursue the matter at that time. According to Redinger and Levine (1998, p. 573), many enterprises have not been deterred by this and have been organising their OHS and Environmental Management functions along the lines of the ISO 14001 model. Other key guidance models are those provided by the US Occupational Safety and Health Administration (the OSHA Voluntary Protection Program), the British Standards Institute (BS 8800: 1996), in conjunction with the British Health and Safety Executive's OHS Guidelines (HS(G) 65), and the American Industrial Hygiene Association's OHSMS (Gerhardsson, 1998, p. 7; Redinger \& Levine, p. 573). 
According to Deacon and Pearce (1992), the Health and Safety Executive's HS(G)65 publication "Successful Health and Safety Management" provides a conceptual model of how a successful safety management system should be conducted (Figure 2).

The British contribution promotes the use of auditing to test the organisation's safety management system against the six elements listed, namely policy, organising, planning and implementation, measuring performance, reviewing performance, and even auditing practices within the organisation (Deacon \& Pearce, 1992, p. 14).

According to Standards Australia (2000, p. 3), an effective OHSMS can assist an organisation to:

- Set out OHS policy and objectives.

- Establish, assess and review the effectiveness of procedures that give effect to OHS policy and objectives.

- Achieve conformance with OHS policy and objectives of the organisation.

- Demonstrate such conformance to others.

Stephan (2001, p. 247) suggests that: "a properly implemented safety management system will act similarly to an organisation's quality management system in that it will act as a wedge to decrease the rate of performance decay over time." According to him, such a system should exhibit the following attributes:

- Management commitment, with visible and active leadership from senior management.

- A supportive organisational culture that secures involvement and participation at all levels with positive reinforcement.

- The integration of safety into the overall management system.

- Effective communication with all employees.

- A planned approach to the identification, assessment and control of hazards.

(Stephan, 2001, p. 247) 


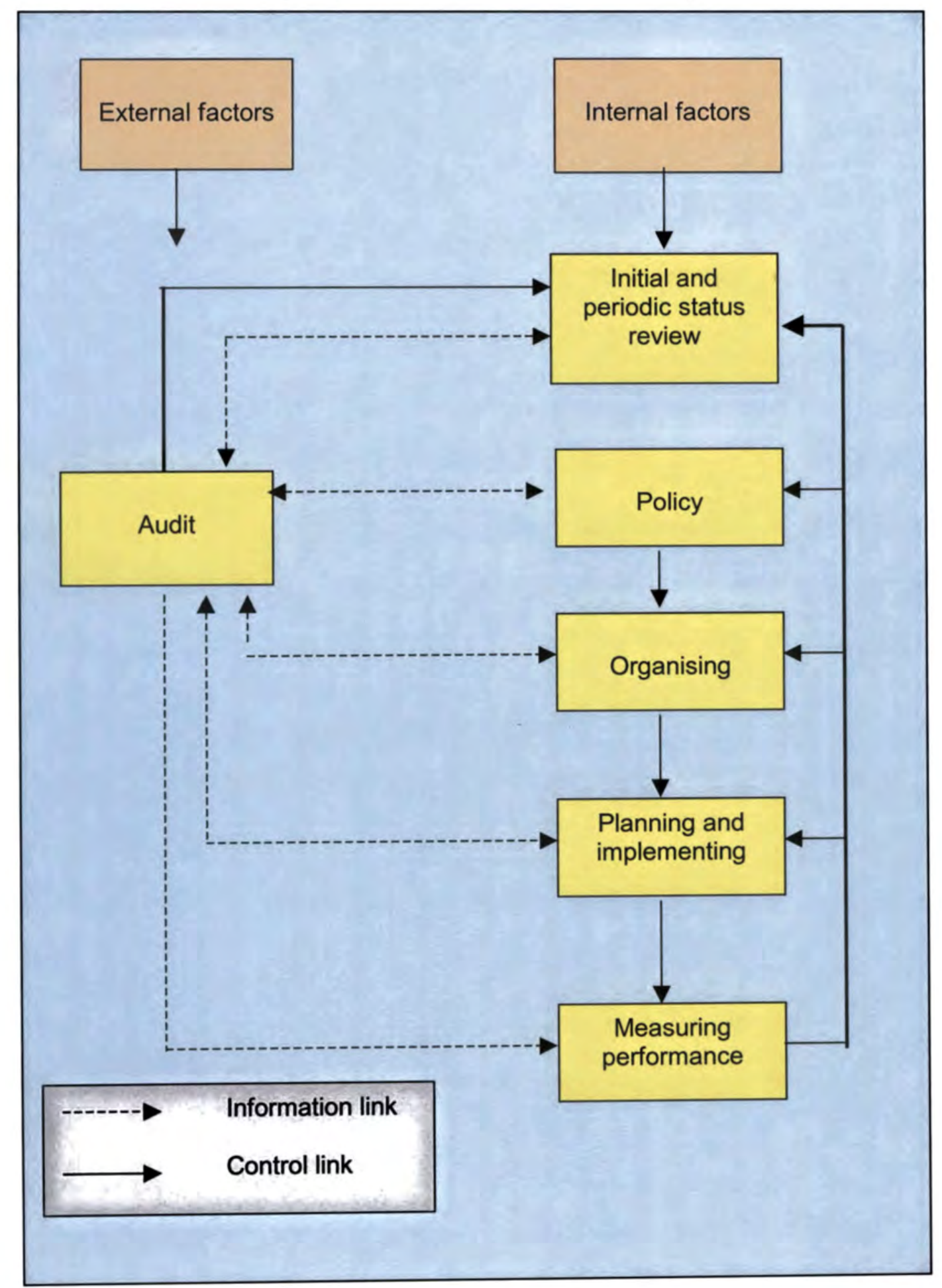

Figure 2: Elements of successful OHS management (Based on HS(G)65 (1993) and cited in Lindsay (1992, p. 391)). 
The National Occupational Heaith and Safety Commission (2001, p. vii) warns that there are three key barriers to success with OHSMSs. These are:

- Failure to customise systems to organisational needs, imposition without consultation, weak senior management commitment and poor employee involvement - all necessary conditions for success.

- Inappropriate use of audit tools - where they may become an end in themselves and are conducted without sound auditor skills, standards and criteria.

- Application in "hostile" contexts, such as that provided by some small business situations, precarious employment, contractors and labour hire companies.

Winder (1997, p. 34) suggests that an OHSMS should have the following attributes:

- Be consistent with legislative compliance as a minimum.

- Be consistent with OHS policy.

- Be aimed at significant workplace risks.

- Have options for control/prevention.

- Specify the requirements of the organisation (eg. business, shareholders, the public).

Along similar lines, Roughton and West $(2000$, p. 27) suggest that the following elements are part of a successful safety effort:

- Management commitment to achieving safety objectives.

- A culture of safety awareness and motivation at all levels.

- A safety program that is rewarding, entertaining and easy to understand.

- A focus on safety every day, as opposed to only when an injury occurs or when bonuses are due.

- Healthy peer group pressure.

- A program that is visually dynamic, flexible and involves recognition.

- Promotion of employee accountability, communication and individual incentives.

- Encouragement of management/employee cooperation. 
- Rules enforcement, including the rule that all injuries and near misses, as well as hazards and at-risk behaviours, must be reported.

Gallagher (cited in National Occupational Health and Safety Commission, 2001, p. vii) states that success with OHSMSs is dependent on factors such as "the kind of system used, senior management commitment, integration into general management systems and effective employee participation".

The Esso Longford Gas Plant accident that occurred in Victoria in September 1998 focused attention on safety management systems. The report arising from the Royal Commission investigation that followed this major accident was extremely critical of the Esso/Exxon Operational Integrity Management System - both in terms of its complexity and clarity, and with the various failures attached to its deployment (Chesson, 1999, p.1). A myriad of legal proceedings followed the incident and the Company was heavily fined in the Supreme Court. The judge made the interesting obsersation that "to use the term 'accident' denotes a lack of understanding of responsibility and a lack of understanding of cause" (cited in Blake, Dawson, Waldron, 2001).

In the UK, failure of management systems for OHS has been a consistent conclusion in many incident inquiry reports - for example, those relating to the Piper Alpha, King's Cross and Herald of Free Enterprise disasters (Deacon, 1992, p. 14). In these cases, management decisions led to:

- Unrealistic timescales for the implementation of plans.

- Fatigue issues associated with work scheduling and rosters.

- Inadequate training.

- Organisational restructuring which placed people in positions for which they had insufficient experience.

- Tasking and control systems which failed to allow for human error and communication difficulties.

(Health and Safety Executive, 1992, p. 11)

Pidgeon (1991, p. 130) nctes that such incidents serve to "focus attention on the human and organisational elements that might contribute to the 
unsafe operation of technological systems". He refers to the notion of a socio-technical system, with close interdependence of both the technological artifacts and behaviourai resources (individual, group and organisational) necessary for the operation of any large-scale technology (Pidgeon, 1991, p. 131). With respect to disaster outcomes, he notes that these have the following general characteristics: the causes are multiple over time, qualitatively diverse, and compounded in complex, interactive ways (Pidgeon, 1991, p. 131).

As mentioned earlier, in Australia, quality management is addressed in AS 3000-3004. Occupational health and safety is dealt with in a pair of linked and complementary standards. These are AS/NZS 4804: 1997 ("Occupational health and safety management systems - general guidelines on principles, systems and supporting techniques") and AS 4801: 2000 ("Occupational health and safety management systems specification with guidance for use"). The first provides general advice on how to develop and implement an occupational health and safety management system. The second establishes an audit framework, principally for use by third party bodies that have been asked by an organisation to conduct an independent audit of its operations (Standards Australia, 2000, p.1; Winder, Gardner \& Trethewy, 200i, p. 70).

\subsubsection{Behavioural safety}

Winston Churchill (cited in Jay, 1996, p. 92) once said: "the empires of the future are the empires of the mind." Many organisations around the world have made great progress with the development of policy statements and procedures, engineering controls, personal protective equipment controls and the like, but have failed to place due emphasis on the "empires of the mind". That is, they haven't exploited the improvements to safety and health that are possible when the drivers of human behaviour are taken into account and suitably addressed.

Petersen, 1991, p. 49) puts it that "in most cases, unsafe behaviour is normal human behaviour: it is the result of normal people reacting to their 
environment. Management's job is to change the environment that leads to the unsafe behaviour."

Enterprises that display excellence in their safety and hesaith effort usually have a behavioural safety component in their programs. Understanding the factors that shape an individual's behaviour provides a basis for the organisation to move more efficiently towards its safety goals (Krause, 2000, p. 475).

Marcombe, Krause and Finley (1993, p. 15) see the behaviour-based approach to safety as focused on observable, measurable behaviours that are critical to safety at the facility - witti such behaviours constituting critical work-related skills.

Krause, Hidley and Hodson (1991, p. 50) link behaviour-based safety management with quality improvement. They identify eight quality concepts with behaviour-based accident prevention:

- Constancy of purpose (long-term strategies).

- Process, not program.

- Do it right the first time.

- Do not blame the employees.

- Specify standards in operational terms.

- Use measurement of upstream factors to assess performance.

- Improve the process, not the downstream results.

- Use statistical techniques to distinguish variation due to "common cause" from variation due to "special cause".

(Krause, Hidley \& Hodson, 1991, p. 50)

Topf (1997, p. 31) states that an integrated, comprehensive behaviour management process is the tool to achieve lasting change, while Krause $(2000$, p. 478 ) asserts that the behavioural process becomes a method for continuously improving facilities, equipment, design and management systems.

Ragan (1997, p. 27) suggests that human error is a fundamental cause in 100 percent of incidents and that it is manifest in all three business 
processes: human, work environment and organisation. Thus, "if accidents are the result of system failures, all components of that system are involved."

A paper in the Eivironmental Manager (1995, p. 12) deals with human error management. It is pointed out that avoidance of human error is fundamental to process safety. Furthermore, research shows that human error is a significant factor in almost all catastrophic accidents in the chemical and petroleum industries (and in others). Human errors, as root causes of accidents, have been found at all stages in a process - including siting, design, operations, maintenance and management (Environmental Manager, 1995, p. 12).

In a similar vein, Geller (1997a, 1999a-g) advocates a systems approach. He considers that at-risk behaviour contributes to 95 percent or more of most injuries, whether intentional or unintentional. As mentioned earlier, he emphasises that outcomes are influenced by environmental, behavioural and personal factors, and that these elements are interactive, dynamic and reciprocal. For example, when people choose to change their behaviour, they adjust their attitudes and beliefs (personal factors) to be consistent with their actions (Geller, 1997a, p. 2).

According to Fulwiler (1998, p. 26), "the application of the principles and concepts of behaviour-based management systems to safety is growing like wildfire." The DuPont organisation (cited in Department of Minerals and Energy, 1995b, p. 10), recognised worldwide for its safety excellence, put it that "the workplace is never really safe. It is the behaviour of people in the workplace that determines whether or not injuries occur."

Reynolds (1998, p.8) and Stephan (2001, p. 244) reflect a widely held view that organisations wishing to adopt behavioural safety techniques need to understand the basic $A B C s$, that is, antecedents, behaviours and consequences. This is represented in Figure 3. Antecedents are seen as the triggers or activators of safe or unsafe behaviours, while consequences, both positive and negative, need to be established to encourage safe behaviour and discourage unsafe behaviour respectively 
(Reynolds, 1998, p. 8). "Activators and consequences are either naturally present in the environment or are created by the safety management system and are added to the environment in order to change or sustain desired behaviour" (Stephan, 2001, p. 244). Thus, signs, policies, procedures, safety culture and training are all activators of behaviour as they indicate potential consequences (Stephan, 2001, p. 244).

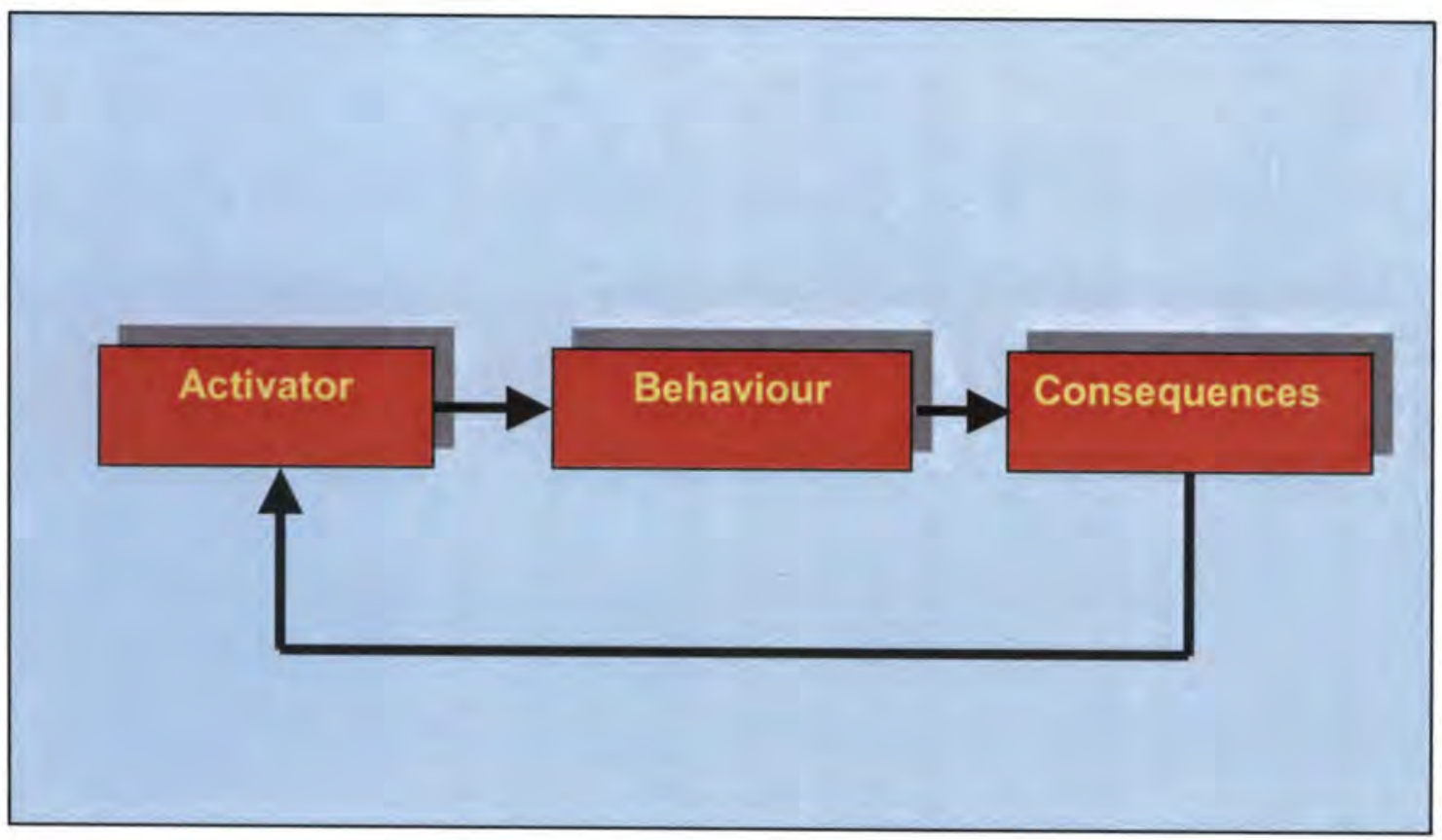

Figure 3: The ABC model of human behaviour (from Stephan, 2001, p. 244)

On the matter of consequences, Petersen (1991, p. 49) notes that, "behaviour change comes quicker and lasts longer using positive reinforcement than with discipline and punishment".

Geller (1997a, p. 4) proposes an approach built upon seven principles:

- There is no single root cause of an incident or injury.

- Environmental, behavioural and personal factors need to be considered.

- These factors need to be measured. 
- Invristigate facts, not faults.

- Feedback directs and motivates.

- Consistency develops commitment.

- Reciprocity should be embraced.

Geller (1997a, p. 4) strongly advocates "active caring" behaviour as the mechanism to provoke reciprocity. The recipient of "active caring" is likely to return the favour to someone else. Further, Geller has coined the term "total safety culture". This focuses on the connection between activators, behaviours and consequences (Geller, 1997a, p. 4).

Topf (1997, p.30) points out that there are two primary types of behaviour that may give rise to injury. These are:

- Unconscious or automatic behaviour, characterised by inattention, and

- Conscious or deliberate behaviour, characterised by shortcuts and other types of calculated risk-taking.

He asserts that these behaviours are "the end products of a powerful web of unproductive attitudes and beliefs on the part of employees, and unproductive actions on the part of leader" (Topf, 1997, p. 30). Behaviour is the product of two sets of forces. Firstly, there are the drivers that exist within the individual. These take the form of personal attitudes, beliefs, values and motivation to perform safely. Secondly, there are external drivers - the physical environment, organisational systems and processes (development of procedures, audits, training and similar), and organisational culture. The latter is an important antecedent in that it embraces the shared values, group norms of behaviour, work practices and climate of the enterprise (Inwin, 1994, p. 3). Business leadership expert John Kotter, in reviewing the power of culture, defined shared values as "important concerns and goals shared by most people in a group that tend to shape group behaviour and that often persist over time, even when group membership changes" (Kotter, 1996, p. 148). He described norms of behaviour as "common or pervasive ways of acting that are found in a group and that persist because group members tend to behave in ways that teach these practices to new members, rewarding those who fit in and sanctioning those who do not" (Kotter, 1996, p. 148). 
Pidgeon (1991, p. 135) notes that "a culture is created and re-created as members of it repeatedly behave in ways that seem to them to be natural, obvious and unquestionable ways of acting, and, as such, will serve to construct a particular version of risk, danger and safety".

Krause and Hidley (1989, p. 21) put it that the culture (or shared common values) in an organisation is the most powerful determinant of how employees behave in relation to risk. A new employee soon assesses how the organisation functions, how iellow employees behave and what behaviour patterns are tolerated, encouraged or frowned upon. Krause, Hidley and Hodson (citet in Dial, 1992, p. 40) assert that culture gives rise to management systems and that these systems either create or eliminate the exposures that can lead to accidents. Winder $(1995, p .216)$ summed it up with a view that, where safety has a high priority, the climate within the organisation is likely to be positive, with safety consciousness permeating through the entire organisation.

But change is rarely immediate. Waring (1991, p. 22) points out that any fundamental shift in an organisation's safety culture decided by senior management is likely to take years to complete. In the aftermath of the Piper Alpha disaster in the North Sea, it was envisaged by many that a 510 year time frame was needed for the necessary safety culture shift.

Leadership is important. Dial (1992, p. 44) states that "transformation begins at the top", while Kotter (cited in Toca and Woodhull, 1996, p. 22) sees management's role as bringing order and consistency to key dimensions of the organisation - such as standards, quality, guidelines and procedures. Indifference to safety by senior management can lead to minimal standards (Winder, 1995, p. 216). Brandt (1997, p. 28), in dealing with managing change in health and safety said, "the strength and conviction demonstrated by the leader will set the pace and direction of the team." Kotter (1999, p. 25) expressed a similar view with: "Leadership defines what the future should look like, aligns people with that vision, and inspires them to make it happen despite the obstacles." 
Young and Prud'homme express leadership in a slightly different way. According to them, in a pro-active culture "everybody is a leader" and that "leadership has nothing to do with roles" (Young \& Prud'homme, 1991, p. 93).

According to Petersen (cited in Johnson 1988, p. 23), "the key to effective line safety performance is management procedures that fix accountability." In a later publication, Petersen (1997, p. 45) elaborates on the importance of management accountability. He sees this as being the key to building a culture that states: "safety is so important that all managers must do something about it every day." When employees believe this, their behaviours will follow.

The Department of Minerals and Energy (1998, p. 10) emphasises that it is "the actual, not the advertised, management practices which register with employees."

Culture can be particularly damaging if it encourages unsafe behaviour or reinforces beliefs that run counter to safety. Examples of the latter are found in the common beliefs that safety and productivity are mutually exclusive (and that the operator must frequently make a choice between the two), that the design and delivery of safety programs, together with enforcement, is the responsibility of someone else (often the safety professional) and that serious accidents always happen elsewhere (Quinlan, 1999, p. 20; Ralph, 1999, p. 6).

According to Kotter, shared values tend to be deeply ingrained in the culture of the organisation and are more difficult to change than norms of behaviour (Kotter, 1996, p. 148).

Individual attitudes represent another key antecedent. Attitudes are enduring positive and negative thoughts and feelings that affect the individual's outlook and perceptions about the work er:vironment and the people within it. Pidgeon (1991, p. 136) put it that "safety attitudes refer to individual and collective beliets about hazards and the importance of safety, together with the motivation to act on those beliefs". 
A common assumption is that bad attitudes cause accidents and that a focus on attitude will result in employees acting more safely. However, attitudes are difficult to change. It is more productive to concentrate on modifying behaviour (Marcombe, Krause \& Finley, 1993, p. 15).

A further, closely related antecedent is morale. When elevated, morale energises people and brings out their best in terms of OHS performance. Lindsay, Manning and Petrick (1991, p. 100), one of the prominent research groups in this area, have defined morale as "the attitude of an individual, group or organisation with regard to the function or task at hand." They observe that a workgroup with high morale has a strong sense of shared direction and a commitment to peak performance. In the workplace, morale is a function of an individual's attitude to the job, interactions between the workgroup, management practices and economic rewards. Geller (1997b, p. 43) notes that: "Giving and receiving recognition are prime ways to boost morale. Likewise, properly executed celebrations can boost morale, motivate teamwork and promote a sense of belongingness."

The studies by Lindsay, Manning and Petrick revealed a strong link between morale and the financial performance of the organisation (Lindsay, Manning \& Petrick, 1991, p. 106). In the OHS area, when management demonstrates its commitment to the safety, health and welfare of the workforce, this is usually a boost for morale. In turn, high morale is linked to safe behaviour - provided that training, equipment and people resources are adequate and there are effective supervisory practices in place.

On the matter of consequences, if an individual receives positive reinforcement from co-workers in relation to a particular behaviour (good or bad), then this is a powerful motivator. Dial $(1992$, p. 43) put it that "the natural work environment frequently generates consequences that reward unsafe behaviour and deter safe behaviour." Peer pressure is one of the most powerful motivational tools known (Roughton \& West, 2000, p. 30). So, the challenge then is to identify what consequences are motivating or 
are capable of motivating the workforce. Once identified, both the positive and negative consequences can be applied directly or in modified form to improve performance. Examples of positive consequences are praise from the supervisor or manager, social approval from co-workers, a good performance appraisal and financial or non-financial forms of reward or recognition (Brandt, 1997, p. 28). Furthermore, "the recognition and reward system of the organisation must reflect what is valued in the new culture" (Brandt, 1997, p. 26). Examples of negative consequences are disciplinary action or other forms of disapproval from the supervisor, as well as negative feedback or ridicule from fellow workers. Perdue $(2000$, p. 3) refers to studies that have demonstrated the role of peer pressure in reducing safety shortcuts (and injury outcomes).

Roughton and West advocate incentive programs as a means of enhancing positive consequences. They envisarge this as part of a broad approach that addresses activities and performance, hazard communication and rules enforcement, behaviours and attitudes, awareness and motivation (Roughton \& West, 2000, p. 29). An effective incentive program will capitalise on the impact of positive group dynamics as a means of shaping individual attitudes and behaviours (Roughton and West, 2000, p. 30). They point out that behaviour-based safety addresses a change in behaviours, with the outcome being an expected change in attitudes. This contrasts with incentive programs that place emphasis on changing attitudes, with a change in behaviour as the expected outcome (Roughton \& West, 2000, p. 28).

Quinlan (1999, p. 20) casts safety incentive schemes in a more negative light with his suggestion that these may enhance or encourage deviant behaviour rather than producing genuine improvements in the OHS area.

An important initial step in putting in place a behavioural safety program is to identify observable (and measurable) key safe behaviours that lie at various points upstream in the process, that is, upstream of incidents, accidents or other outcomes (Stephan, 2001, p. 244). 
management. According to Krause, Hidley and Hodson (1991, p. 50), "the strongest reinforcement for behaviour is feedback that is soon, certain and positive [emphasis added]." Systematic job/task observations are becoming popular. Trained co-workers usually conduct these since "shop floor personnel are in constant contact with a site's production processes and with the sheer mass of workforce behaviours" (Krause, 1997, p.25). They provide coaching feedback on both safe and at-risk behaviours and this occurs in a formalised but non-threatening way. This "success" feedback and "guidance" feedback must be provided face-to-face. The one-on-one dialogue between peers is instrumental in changing at-risk work practices. It allows the observer and the observed to analyse the situation together to identify and remove any barriers to safe work performance (Perdue, 2000, p. 2). Furthermore, the observation data from the individual work sheets are collated, analysed to identify areas for follow-up action and then shared with a wider group of employees as feedback (Perdue, 2000, p. 2). Management 's role, according to Krause, is "to establish a support system so that all employees may sample safety performance using operational definitions and analyse resulting data to identify barriers to continuous improvement" (Krause, 1997, p. 25).

In a benchmark behavioural program, employees will routinely observe each other while working. Full commitment would be represented by:

- Employees actively cautioning co-workers obscrved dernonstrating atrisk behaviours.

- Employees asking co-workers to caution them when they are observed performing at-risk behaviours.

(Geller, 1998, p. 147)

Stephan (2001, p. 245) sees the provision of supportive and corrective feedback by one worker to another, and the acceptance of that feedback as a critical sign of a healthy safety culture. He recognises that more formalised job observation processes may be required in the early stages. Ultimately, however, "by providing training in behaviour observation and feedback and by providing a system whereby individuals and teams are accountable for conducting regular feedback sessions, the safety culture 
could advance to a situation where the formal processes are not necessary" (Stephan, 2001, p. 245).

Krause (2000, p. 479) notes that behavioural data is an important element on which to develop action plans for improvement. Such data takes the focus from the worker and puts it on systems - including facilities, equipment, design, maintenance and more subtle mechanisms such as purchasing, decision-making and other managerial functions. Thus, behavioural observation data needs to be analyeed to identify barriers to safe work and the factors that prevent workers from performing their tasks in a safe manner. The subsequent step is to formulate action plans to remove barriers (Krause, 2000, p. 479).

However, behavioural safety initiatives of this kind will only succeed where there is a good employee-relations climate and a strong element of trust between management and the workforce. Where these conditions exist, employees should be trained in the principles of behaviour modification, given examples of at-risk behaviours and provided with the opportunity to develop their observation and feedback skills. Goals must be set in a participative manner and not imposed by cthers. Collective goal-setting ard identification of improvement opportunities are enhanced via group feedback sessions on performance (Geller, 1997b, p. 41).

On the matter of participation, Mitchell (1993a, p. 3) points out that "we are more likely to modify our own behaviour when we participate in problem analysis and solution" and, furthermore, that "we are also more likely to carry out decisions we have helped to make because our motivation is linked to our ability to have a direct influence on results" (Mitchell, 1993a, p. 3).

Krause (1997, p. 21) notes that "fims that have done it well - establishing behaviour-based safety as a self-sustaining process - have found that the task requires the resolution of central organisational issues." He points out that the task is a unique undertaking at each location and that it is always necessary to adapt the behaviour-based method to the culture at the facility (Krause, 1997, p. 21). 
Ragan (1997, p. 27) sounds a caution in noting that behavioural safety programs that dwell on job observation are concerned with only one system element and occur only after the decision to act has been made. He points out that measurement is taking place only one step before the accident, rather than several steps before.

In similar vein, Roughton and West $(2000$, p. 30$)$ point out that many injuries result from simple carelessness that cannot be traced to a specific behaviour. A worker who has demonstrated safe behaviours for years can have a bad day - with stress, lack of sleep, emotional strain, minor illness, substance abuse or similar helping to create an injury outcome.

Trethewy, Gardner, Cross and Marosszeky (2001, p.253) and the Environmental Manager (1995, p. 12) also wam against over-emphasis on human behaviour at the expense of accounting for environment and system causes. Workplace culture that has been moulded by management is seen as a key determinant of safety outcomes.

Shaw (1994, p. 18) provides a blunt assessment along similar lines. Like Ragan, she suggests that the behavioural approach means measurement is only one-step removed from system failure (in this case, injury or disease) and does not actually address the circumstances that give rise to unsafe behaviours.

Geller (1997a, p. 3) adds a further dimension to the subject of feedback and its role in motivating and activating safe behaviour. He suggests "that the natural feedback from convenience, comfort or a faster outcome usually competes with the completely safe way to do work." Thus, systems thinkers need to address these matters and use feedback as an activator to direct improvement in particular work practices.

Krause, Hidley and Hodson (1991, p. 49) highlight the importance of developing an inventory of critical safety-related hehaviours for the facility. Unfortunately, where this has been done there is often undue emphasis on the behaviour of shopfloor employees, and scant regard to establishing, 
measuring and reinforcing the behaviours that are needed from line management.

Unfortunately, many of the components of traditional safety programs, that is, safety contests, slogans, exhortations, poster programs, and similar exert little impact on behaviour. In particular, they do not address the effects of social reinforcement for unsafe behaviour and at times may be counter-productive (Ralph, 1999, p. 6; Quinlan, 1999, p. 20).

Kotter (1999) has highlighted the need to anchor changes firmly in the corporate culture. He points out that until new behaviours are rooted in social norms and shared values, they are always subject to decay as soon as the pressures associated with a change effort are removed (Kotter, 1999, p. 14).

Topf (1997, p. 31) examines the prerequisites and features of breakthrough safety. According to him, break-through safety occurs as a largescale cultural shift that has a positive impact on every aspect of the organisation. It occurs when there is acceptance of personal responsibility by every member of the organisation. Furthermore, it occurs when employees internalise the message: "My well-being is not the job of the safety manager. It is my job, above and beyond everything else I do here." He suggests that, while it is natural to be drawn towards quantitative measures, there are a number of qualitative measures that are worth examination. These include:

- Increased participation in safety meetings.

- Greater use of personal protective equipment.

- Better acceptance of feedback from management and co-workers.

- Enhanced communication regarding safety and environmental issues between line employeas and management, and among team members.

- Greater willingness of employees at all levels to behave pro-actively in safety.

- More frequent reporting of observed hazards. 
- Visible evidence of increased concem and caring for co-workers' safety.

Pidgeon has a slightly different view on behavioural safety. He suggests that a broader view should be taken of behavioural contributions to accident incubation (and outcomes). He sees this as "ranging from simple, individual errors, such as slips or mistakes, to those associated with social arrangements, either involving inter-group or intra-group communication failures, or those more deeply rooted in large scale organisational information systems and dispositions" (Pidgeon, 1991, p. 131).

The Liberty Mutual Group, a world leader in safety, has a consultancy arm that conducts corporate climate reviews. These provide an in-depth look at the norms, beliefs, roles, and social and technical practices that characterise an organisation's operating environment (Liberty Mutual, 2000 a, p. 1). Their review efforts are directed at employees and management, and cover organisational commitment, job satisfaction, personal accountability, management commitment, worker involvement, co-worker support, training/equipment/environment and performance management (Liberty Mutual, 2000a, p. 1).

The same organisation also provides a service to establish the readiness of a site to embark on a behavioural safety program (Liberty Mutual, $2000 \mathrm{~b}$, p. 1) and a service to map progress in implementing the various modules that make up the behavioural safety effort (Liberty Mutual, 2000c, p. 1).

Bob Bea (1999) has studied higher reliability organisations (HROs) and their ability to operate reiatively error-free over long periods of time, and in many cases, in very hazardous environments. He has observed that such bodies use extensive process auditing procedures to help identify safety problems and they have reward systems that encourage risk-mitigating behaviours. According to him, individuals and groups operate within a quality space that is enclosed by four boundaries - physical, psychological, social and economic. People continually move within that 
space, responding or reacting to warnings as they approach the boundaries of acceptable behaviour. He points out that these boundaries are susceptible to management and change (Bea, 1999, p. 3). He advocates the design of people-friendly engineering and management systems that give the workforce large latitudes in their behaviours and still maintain a safe or acceptable state. He calls for systems that clearly indicate when the boundaries of safe behaviour are being approached and provide sufficient information and time to allow the deficiencies to be recognised and corrected. Finally, he points out that boundaries can be provided that destabilise slowly and are not "brittle" (where crossing the boundary immediately leads to "failure" (Bea, 1999, p. 4).

Waring (1991, p. 22) identifies the interpretive or human factor subsystems as being more likely to cause problems than functional components. He comments that engineering controls usually do their job relatively quickly, thus creating an unrealistic expectation that all control systems will function this way.

Kletz has been advocating since 1976 that human failure rates need to measured and understood in the same way that this is done for equipment failure. He points out that there are four key reasons why human error manifests itself as a mishap. These are:

- People do not know what to do (lack of training or instruction)

- People know what to do but decide not to do it (lack of motivation)

- People find the task is beyond their physical or mental ability, or

- People have a momentary slip or lapse of attention and fail to carry out an action (or carry it out incorrectly).

(Kletz, 1993, p. 411)

Failure rate data is available for the last category, but collection is much more difficult for the first three categories. These tend to be more sitespecific and relate to issues such as quality of training and instruction, quality of supervision and level of motivation, and task and workplace design (Kletz, 1993, p. 412). 
It would seem that the behavioural safety approaches described above, including job observation initiatives, tend to address the first two elements. The third and fourth are planning and design issues, and need to be addressed differently. These are related to matters such as fitness for work of the individual, patterns of work, ergonomic design, equipment maintenance and to task design that requires unfailingly accurate performance from human beings (Kletz, 1993, p. 413).

James Reason has alluded to this with his concept of latent and active failures (cited in Amis and Booth, 1992, p. 45; Hurst, 1998, p. 26). Reason argues that latent failures made by managers lie dormant in an organisation for some time until triggered by active failures, usually made by the people directly at risk. Furthermore, he (Reason) suggests that safety professionals should direct their efforts to dealing with latent failures, rather than the prevention of active failures, as they have largely been in the past.

As Topf (1997, p. 31) put it, when the fundamentals are addressed and there is a significant change in attitudes and beliefs, then "the benefits reverberate throughout, and beyond, the organisation." Furthermore, "Ineffectiveness, waste, injuries, environmental incidents and workers' compensation costs decline, while creativity, morale, productivity, and onand off-the-job safety increase." However, he warns that addressing behaviours alone, without dealing with what underlies and drives them, can be a slow and costly exercise.

\subsubsection{Risk Management}

Heavy industrial workplaces are usually associated with a variety of physical and chemical hazards - all of which need to be recognised, evaluated and controlled (Harvey, 1980). This is the area of risk management. Standards Australia (1999, p. iij) sees risk management as "an iterative process consisting of well-defined steps which, taken in sequence, support better decision-making by contributing a greater insight into risks and their impacts". To be most effective, it must be integrated into the organisation's philosophy, practices and business plans rather 
than be viewed or practiced as a separate program (Standards Australia, 1999, p. iii).

In Australia, risk management, in its simplest form, is considered to involve the specific program elements of hazard identification, risk assessment and risk control (Winder, Gardner \& Trethewy, 2001, p. 70). Standards Australia takes this further in AS/NZ 4360: 1999 with a risk management process as depicted in Figure 4. The main elements are as follows:

- Establish the strategic, organisational and risk management context in which the rest of the process will take place.

- Identify what, why and how things can arise as the basis for further analysis.

- Determine the existing controls and analyse risks in terms of consequence and likelihood in the context of those controls.

- Compare estimated levels of risk against the pre-established criteria. This enables risks to be ranked and priorities to be identified.

- Develop and implement management plans for higher-priority risks.

- Monitor and review the performance of the risk management system and changes that may impact on it.

- Communicate and consult with internal and external stakeholders at each stage in the process, as appropriate.

(Standards Australia, 1999, p. 7)

Within Western Australia, the Mines Occupational Safety and Health Advisory Board has produced a risk management guideline for the Mining Industry (MOSHAB, 1999). Its content is consistent with that of AS/NZ 4360: 1999. 


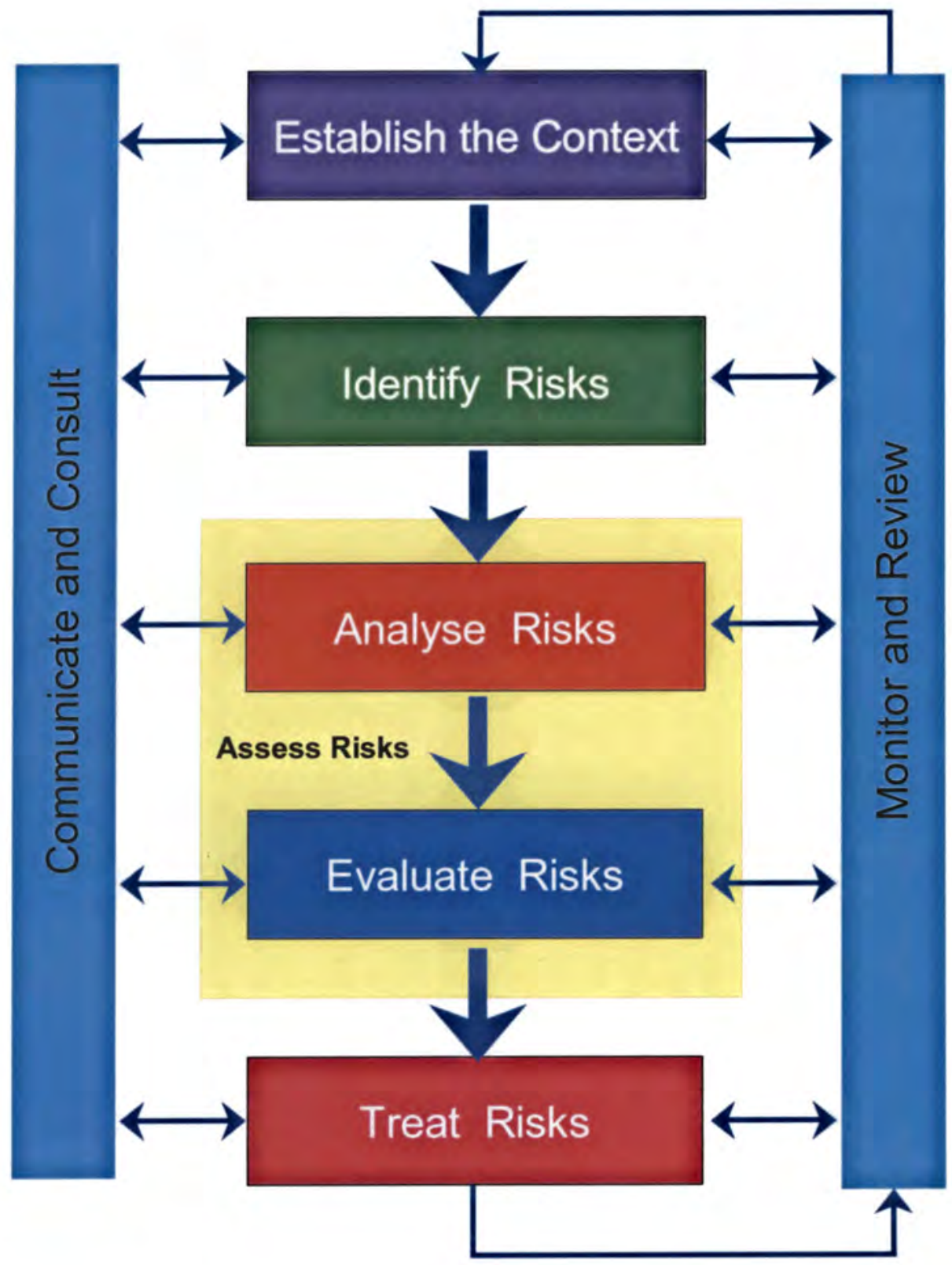

Figure 4. The risk management process

(Standards Australia, 1999, p. 11)

\subsubsection{Hazard identification}

Human health and safety risks may originate from materials in use, production processes, products, by-products, wastes, packaging, 
distribution and disposal functions, and may impact on workers, the community and customers (Alcoa, 1997, p. 4).

According to the UK's Health and Safety Executive (1992, p. 39), it is necessary to assess the nature, extent and relative importance of risks in the workplace. This assists in making informed decisions on the methods of control and suitable allocation of resources and effort.

In discussing risk, Wilson (1999, p. 3) points to the "three whats" approach to questions that must be asked and answered:

- What can happen?

- What is the chance of harm?

- What are the consequences?

The tools available to apply to hazard identification are numerous. These include checklists, general observation, tick-the-box pro-forma sheets, physical inspection, process charting, brainstorming, structured interviews, review of historical data, specialist judgement, incident scenarios, engineering codes and research $(P$. Janus, personal communication, September 4, 2001).

The context (or scope-defining element) mentioned above will involve consideration of the geographical area or process (facility, department, equipment, operating area and similar), the phase of operation (design, installation, start-up; operation, maintenance, shutdown, demolition and similar) and nature of the study - whether it represents a specific hazard or all hazards (Alcoa, 2002.a, p. 3).

\subsubsection{Risk assessment}

According to the Health and Safety Executive (1992, p. 39), the risk assessment process is needed to identify the relative importance of individual risks and to obtain information about their extent and nature. This assists decision-making on the methods of control and the priorities that go with this. Other activities can also be prioritised. These include those associated with further monitoring, training and improving levels of 
competence, review of injury, ill health and incidents, and resource allocation (Health and Safety Executive, 1992, p. 40).

Pidgeon (1991, p. 132) cautions "that the difficulties experienced with the formal assessment of risk are compounded if we adopt a socio-technical view of accident causation". He identifies problems in dealing predictively with the qualitative diversity of accident causes and, in particular, with those of human and organisational origin (Pidgeon, 1991, p. 132): According to Hurst (1998, p. 97), risk assessments are often weak and incomplete because they fail to take due account of organisational factors.

Risk analysis is used to address the relative risk of each identified hazard in terms of potential severity (consequences) and probability (frequency) of an incident arising from a hazard.

On the matter of consequences, these may be positive or negative (however, always negative for safety aspects) and may be expressed qualitatively or quantitatively. There may be more than one consequence from a single event (Alcoa, 2002a, p. 1).

Probability is the likelihood that a specific event or outcome will occur. It is often expressed as a number between 0 and 1 , with 0 indicating an impossible event or outcome and 1 indicating a certain outcome (Alcoa, 2002a, p. 1).

Tables 1 and 2 below provide typical examples of qualitative measures that may be applied to consequence and likelihood respectively (Alcoa, 2002a, p. 6). 
Table 1

Qualitative Measures of Consequence

Level Descriptor Example

1 Insignificant No injuries, low financial loss.

2 Minor First aid treatment, medium financial loss.

3 Moderate Medical treatment required, high financial loss.

$4 \quad$ Major Extensive Injuries, major financial loss.

5 Catastrophic Death (s), huge financial loss.

Table 2

Qualitative Measures of Likelihood

Level Descriptor Description

A Almost certain Is expected to occur in most circumstances.

B Likely

Will probably occur in most circumstances.

C Possible Might occur at some time.

D Unlikely Could occur at some time.

E Rare May occur in exceptional circumstances

A risk analysis matrix may be derived from the measures of consequence and likelihood. This assists with determining the required process improvement solutions. Examples are provided as Tables 3 and 4 (Alcoa, 2002a, p. 7). 
Table 3

Qualitative Risk Analysis Matrix: Consequence and Likelihood

\begin{tabular}{lccccc}
\cline { 2 - 5 } \multicolumn{1}{c}{ Likelihood } & $\begin{array}{c}\text { Insignif- } \\
\text { icant }\end{array}$ & Minor & Moderate & Major & $\begin{array}{c}\text { Cata- } \\
\text { strophic }\end{array}$ \\
\hline Almost certain & High & High & Extreme & Extreme & Extreme \\
Likely & Medium & High & High & Extreme & Extreme \\
Possible & Low & Medium & High & Extreme & Extreme \\
Unlikely & Low & Low & Medium & High & Extreme \\
Rare & Low & Low & Medium & High & High \\
& & & & & \\
\hline
\end{tabular}

Table 4

Qualitative Risk Analysis Matrix: Risk Rankings and Actions

\begin{tabular}{cll}
\hline Risk rank & \multicolumn{1}{c}{ Recommended action } & \multicolumn{1}{c}{ Impact } \\
\hline Extreme & Immediate action. & $\begin{array}{l}\text { Control review plan must } \\
\text { be developed. }\end{array}$ \\
High & $\begin{array}{l}\text { Senior management } \\
\text { attention. }\end{array}$ & As above \\
Medium & $\begin{array}{l}\text { Management responsibility } \\
\text { must be specified. }\end{array}$ & $\begin{array}{l}\text { Control review plan may } \\
\text { be developed. }\end{array}$ \\
Low & $\begin{array}{l}\text { Manage by routine } \\
\text { procedures. }\end{array}$ & \\
\hline
\end{tabular}




\subsubsection{Risk control}

Standards Australia $(2000$, p. 4) notes that the emphasis in legislation and related standards is for "organisations to develop and implement control actions which, whenever possible, eliminate hazards or isolate people from the hazard." Where this is not possible, then administrative means should be applied. The approach prescribed in many legislative instruments and standards refers to a preferred order of control methods, commonly referred to as "the hierarchy of controls" and is represented in Figure 5 below (National Health and Safety Commission, 1994b, p. 49).

The hierarchy reflects that risk elimination and risk control by the use of physical engineering controls and safeguards can be more reliably maintained than those which rely solely on people (Health and Safety Executive, 1992, p. 42).

Organisations should start at the top and work downwards, applying tests of practicability with each control method. Sometimes, combinations of these controls will be the most effective way of dealing with a particular issue. Elimination sits at the top of the hierarchy because it removes the hazard and offers a permanent solution. As Kletz (1993, p. 410) put it: "What you don't have, can't leak." In contrast, personal protective equipment sits at the bottom of the hierarchy because it will depend for its success on being chosen correctly for the task, being fitted correctly and being suitably worn at all times when it is required (Standards Australia, 1997, p.25).

The Health and Safety Executive (1992, p. 38) points out that while health risks arising from the use of substances can be controlled by physical control measures, systems of work and personal protective equipment, the operation of which can be measured, confirmation of the adequacy of control will often require measurements in the workplace to ensure that exposures are within pre-set limits. 


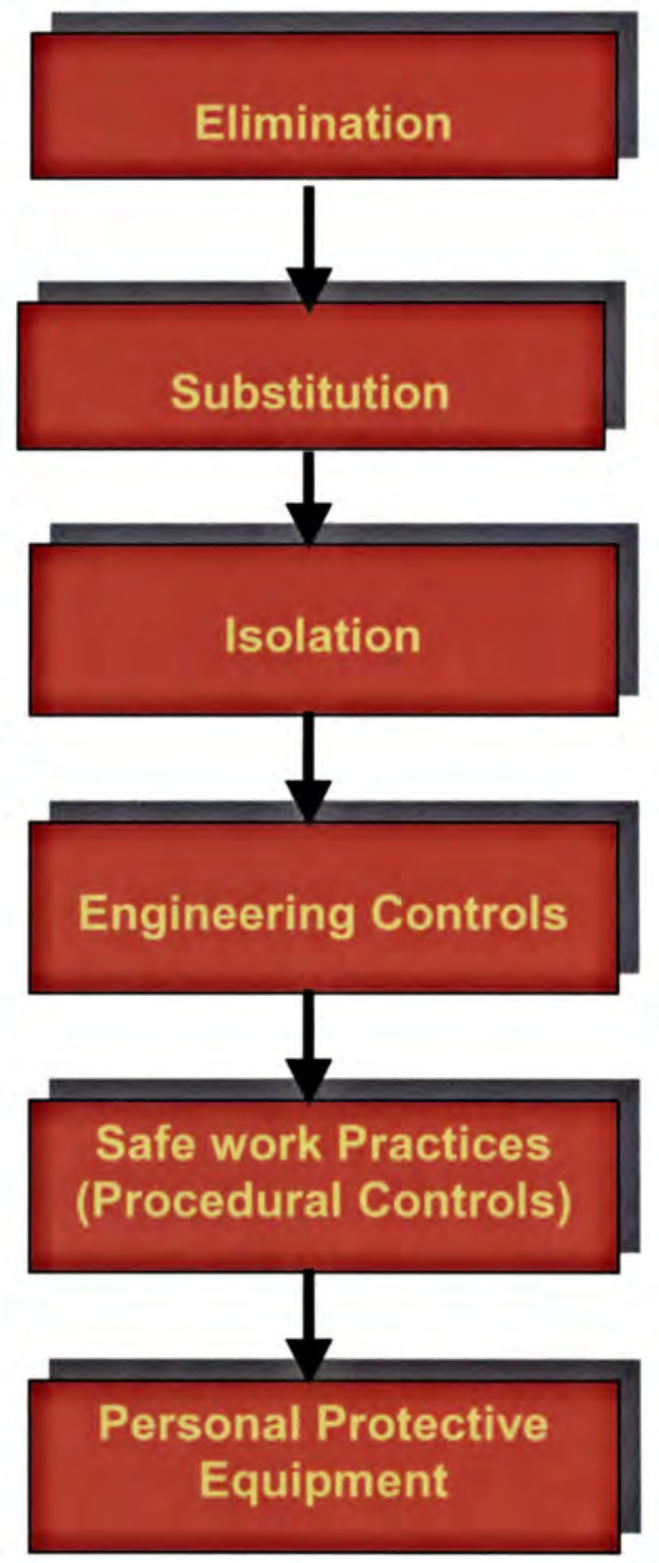

Figure 5: The hierarchy of control measures

(National Occupational Health and Safety Commission, 1994b, p. 49)

Countermeasures may be required as interim controls to reduce risks until long-term solutions can be implemented (Alcoa, 2002, p. 4).

But, the matter doesn't end there. As Lindsay (1992, p. 398) points out, 
"All control systems tend to deteriorate over time or to become obsolete as a result of change." Thus, periodic re-evaluation of the efficacy of the controls is an integral part of the process.

Standards Australia (1999, p. 16) approaches risk treatment from a different perspective. It lists five broad options:

- Avoid the risk by deciding not to prorieed with the activity that carries the risk.

- Reduce the likelihood of the occurrence.

- Reduce the consequences.

- Transfer the risk (via use of contracts, insurance arrangemente, physical transfer to other places, and similar).

- Retain the risk (and manage them).

Here, reduction of consequence and likelihood may be referred to as risk control (Standards Australia, 1999, p. 18).

The Health and Safety Executive (1992, p. 6) makes a different point in asserting that the best efforts go beyond the prevention of injury and ill health at work. They engage in health promotion and thereby support the belief that people are a key resource.

The success of action taken to control risks is assessed via a range of techniques. According to Lindsay (1992, p. 390), this includes an examiriation of both hardware (premises, plant and substances) and software (people, procedures and systems), including individual behaviour. Failures of control are assessed via investigation of any injuries, ill health or incidents with potential to cause harm or loss.

The Health and Safety Executive (1992, p. 29) provides a summary of the elements needed to secure the control of risks. These fit well with what has been described earlier. Key points are:

- Managers who lead by example.

- Clear allocation of responsibilities in areas such as policy development and deployment, planning and reporting on performance.

- OHS responsibilities clearly vested with line management (with specialists acting as advisers). 
- Responsibilities allocated to people with sufficient authority, competence, time and resources to carry out their duties effectively.

- Systems of accountability for individuals, supported by target setting and positive reinforcement.

- The provision of adequate supervision, instruction and guidance.

- Payment and reward systems that avoid conflict between output targets and $\mathrm{OHS}$ requirements.

The Health and Safety Executive (1992, p. 43) points out that in successful organisations the design of risk controls is fully integrated into plant and work design procedures - so that specifications simultaneously satisfy output, quality, and health and safety requirements.

\subsubsection{Implementation issues}

Mitchell puts responsibility for workplace OHS outcomes squarely at the feet of management. In emphasising the safety-quality links discussed earlier, he asserts that "only managers can altocate resources, introduce management processes, provide training, select the equipment and tools to be used and provide the plant and work environment necessary to achieve objectives" (Mitchell, 1993a, p. 3). Furthermore, he adds: "employees, on the other hand, can only be responsible for resolving those special problems caused by actions and events under their control." These are called "Special Causes" in the Quality literature. He borrows from Pareto principles to state that " $80 \%$ of problems that occur result from common causes that stay in the system until management does something about them" (Mitchell, 1993a, p. 3).

Krause $(2000$, p. 480$)$ concludes that since barriers to identified safe behaviours are primarily related to conditions and management systems, rather than personal choice, the focus of safety improvement efforts should be on the systems that enable safe behaviour. He refers to the behaviour-systems interaction as the working interface and calls for this to be systematically defined and improved (Krause, 2000, p. 481). 
Kletz (1993, p. 410) declares that "managers at all levels, including the most senior, should identify the problems that prevent improvement, agree actions and follow up to see they are taken." He points out that this is no more than normal good management, but is often lacking in the safety area.

Kletz raises a further issue in the implementation area - that of corporate memory and the importance of learning the lessons of the past. He points out "that far too many accidents are investigated, reported and forgotten, so that they recur in the same company some years later" (Kletz, 1993, p. 410).

Small business operators encounter many implementation problems in regard to chemical safety. Howell, Spickett and Hudson (1998) have examined the challenges faced within five specific industries in Western Australia. They found that small firms appeared to be more preoccupied with economic survival then OHS and that managers tended to downplay hazards and to emphasise worker responsibility. Furthermore, their contact with external organisations is generally poor, resulting in limited awareness and motivation on OHS matters (Howell, Spickett \& Hudson, 1998, p. 462). They concluded that hazardous substances management standards are low in this sector, but would appear to improve with size of the organisation.

A further matter that bears on implementation is the inherent differences that exist between safety and health. The Healthi and Safety Executive (1992, p. 38) highlights some of the distinctive features that are attached to the latter:

- Health outcomes may result from complex biological processes such as immune responses (asthma), abnormal cell behaviours (cancers) or repair of repeated damage (irritant dermatitis). Also, there is individual variation in response.

- Such processes may occur over many years (diseases of long latency such as asbestos-related disease). Thus, disease outcomes may continue for years after exposure has been controlled. 
- The same disease may be derived from both occupational and nonoccupational sources (eg. asthma, back pain, lung cancer).

- Exposure to disease risk is not always apparent. Measurement of risk factors may be required.

These features suggest that hazard identification and risk assessment activities may require specialist advice, together with the application of occupational hygiene and clinical skills to check that exposures are within acceptable limits and that early signs of harm are detected via health surveillance efforts (Health and Safety Executive, 1992, p. 38).

\section{$2.4 \quad$ Hazardous materials management}

\subsubsection{Hazardous materials - strategic planning and process}

Winder $(1995$, p. 223, 1999b, p. 167) points out that the hazardous materials management effort should be integrated ints, everything that the organisation does. In particular, it should be accommodated by the management functions of planning, organising, leading, coordinating, directing and evaluating workplace systems. In his view, the inclusion of chemical safety into the strategic planning process will bring it into line with all other activities. The linkages that are thereby made will support the establishment of a pro-active effort in chemical safety management.

Winder describes best practice in workplace hazardous substances management. The process to be applied includes gaining a commitment from senior management, putting in place consultative mechanisms, developing a policy for chemical safety and identifying components of a program. The steps of identification and allocation of resources, implementation and review, and integration of the program into the strategic plan will follow this (Winder, 1995, p. 211).

Winder (1999b, p. 163) asserts that, to be effective, the hazardous substances system needs to be set in a conceptual and philosophical framework that facilitates its development. This requires full commitment from stakeholders, deployment of a proactive, risk management approach, tools to allow changes in safety culture and a focus on continuous 
improvement (after initial quantum gains). As Pryor (1989, p. 29) put it: "A briliant strategy will fail unless it is implemented successfully."

\subsubsection{Components of a facility program}

Chesson (1990, p. 406) has described the key components of a hazardous materials management program for a large installation. These are:

- Supply/industrial hygione/medical involvement in vetting new materials and controlling access of the more hazardous substances

- Collation of supplier information, literature information and user experience

- Development of manuals, summary sheets and supplementary systems (videos, posters, placards, interactive displays, newsletter articles and similar) - for awareness- building and training purposes

- Development of systems to monitor deployment and consumption of materials

- Deveropment of procedures and standard work instructions to cover manufacture, transport, storage, use and disposal of hazardous materials

- Posting of waming signs

- Labelling

- Workplace inspections and walk-through surveys

- Industrial hygiene measurements

- Institution of engineering, administrative and personal protective equipment controls

- Medical treatment and surveillance

- Education and training of user groups.

Modem technology has been applied to some of these elements to great effect. For example, Houseman, Behar and LeBlanc (2001) describe the application of bar code technology to the just-in-time management system for use of chemicals at the Jet Propulsion Laboratory in Pasadena, Califomia. Bar coding is used at JPL to keep chemical inventories up-todate, to reduce inventories and to assist with implementation of material 
safety data sheet programs. In their experience, the electronic chemical procurement system provides several advantages over a traditional, paper procurement system. These are:

- Improves the process that prohibits the unauthorised purchase of chemicals and allows safety staff to recommend less hazardous materials, whenever possible, before the chemicals are purchased

- Chemical requests are approved more quickly, with reduced handling costs

- The system provides a downloadable database of delivered chemicals, which can be used to update hazardous inventories

- An electronic document trail is available for all chemical purchases and for the delivery locations

- Users do not overstock chemicals because chemicals can be procured more quickly. This results in lower warehousing costs, less hoarding of chemicals, less chemical spoilage and fewer disposal costs for unused chemicals (Houseman, Behar \& LeBlanc, 2001, p. 28).

\subsubsection{A fishbone for chemical safety}

Cause and Effect Diagrams, also known as Fishbone or Ishikawa Diagrams, were described earlier in relation to the seven tools of the quality approach. Claridge (2002, p. 12) highlights several key attributes of these diagrams:

- They focus attention on one specific issue or problem.

- They allow the various ideas about what the root problem might be to be organised and displayed graphically.

- They show the relativity of various factors that influence a problem.

- They do not have a statistical basis, but are an excellent aid for problem-solving.

- They reveal important relationships among various variables and possible causes.

- They provide additional insight into process behaviours.

- They focus the team on causes rather than the symptoms. 
It is likely that this tool would be very helpful in team-based risk assessments and in other problem-solving efforts in relation to chemical safety. Figure 6 below represents a starting point in dealing with a chemical-related issue in the workplace. The "man", "machine", "methods" and "environment" headings are not fixed, but would seem appropriate in this instance. Typically, team members would "brainstorm" to identify the range of causes to be assembled under the principal headings before moving to remedial measures (Sytsma \& Manley, 1999, p. 1).

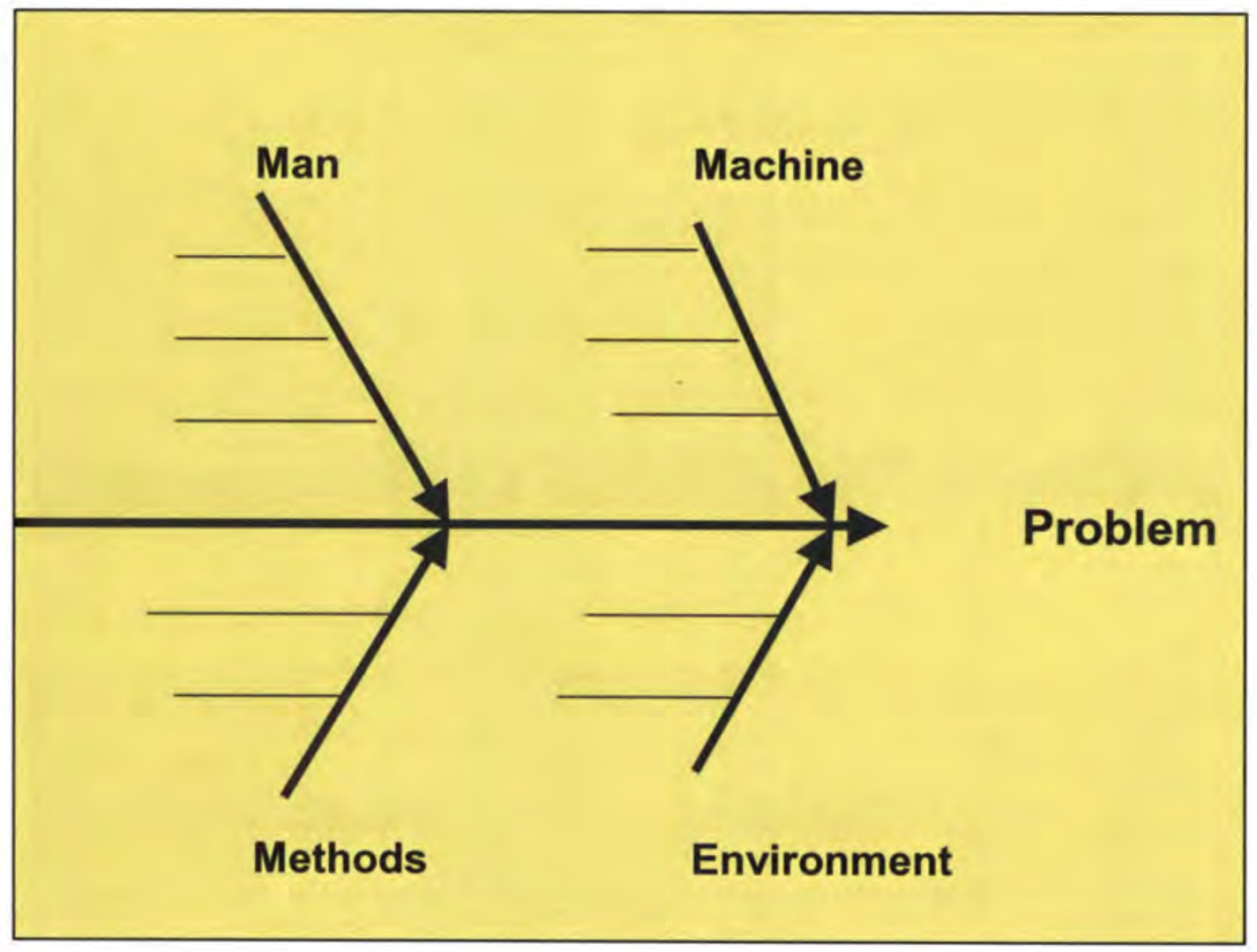

Figure 6: A fishbone for chemical safety

\subsubsection{Implementation issues}

Simple compliance with legislation is a challenge for many industry sectors. For example, Yousiph and Winder studied the ability of the New South Wales electroplating industry to comply with the NSW regulation for 
the control of hazardous substances in the workplace. They found that "most employers in the electroplating industry would not be able to comply with the hazardous substances regulation, with respect to provisions of hazard communication and workplace assessment" (Yousiph \& Winder, 1999, p.150). Further problem areas were identified in relation to monitoring and health surveillance, and in a few instances, with the storage of chemicals (Yousiph \& Winder, 1999, p. 150).

Winder (1999a, p. 102) has noted that there is wide variation in the way employers respond to the problems of chemical hazards in the workplace. Responses range from "the authoritarian to the responsible, to the reactive, to the fragmented, to the negligent. These responses are made more complex by having to comply with a bewildering range of legislation and standards which are confusing and sometimes contradictory" (Winder, 1999a, p. 102). He advocates a risk management approach as the way to deal with these problems - with a framework built around consultation, identification, assessment and control of workplace chemical hazards, and review of the effectiveness of these initiatives.

\subsection{The search for best practice}

\subsubsection{The concept}

The Australian Manufacturing Council $(1994$, p. 1) defines best practice as:

The cooperative way in which firms and their employees undertake business activities in all key processes - leadership, planning, people, customers, suppliers, community relations, production and supply of products and services, and the use of benchmarking. These practices when effectively linked, can be expected to lead to sustainable, world-class outcomes in quality and customer service, flexibility, timeliness, innovation, cost and competitiveness.

Best practice refers to the way in which leading-edge organisations manage their operations to deliver high standards of performance in areas such as cost, quality and timeliness (National Industry Extension Service, 1993, p. 1). Blewett and Shaw (1996d, p. 731) put it that "best practice is an iterative process, with continuous improvement at its heart." Today, 
many organisations use benchmarking techniques and processes to drive their continuous improvement efforts. One of the criteria used in the high profile Malcolm Baldridge National Quality Awards is the presence of an effective program of external benchmarking (Walleck, O'Halloran \& Leader, 1991).

Keeves (1996, p. 1) made the following comment with respect to best practice:

The truth is that the pursuit of best practice is relevant to any business wishing to stay in business. Whether you choose to compare your business with others in your town, your state, nationaliy, or even with the best in the world, the search for best practice can help you to take a fresh look at some of the core processes in your business and possibly make major improvements on them without having to make your own mistakes or having to re-invent the wheel.

The National Industry Extension Service (1993, p. 2) put it that "best practice organisations are improving performance and managing change by actively adapting world class standards to their own needs and capabilities."

Blewett $(1994$, p. 3) has noted "that implementing OHS best practice can help improve management approaches in cther systems as well." This fits with the DuPont philosophy, expressed earlier, that success in managing safety correlates strongly with success in managing the wider business.

Core components of best practice have been described in the International Best Practice Report on the Overseas Study Mission (cited in National Industry Extension Service, 1993, p. 1). When these are adapted to fit a hazardous materials management context, they become:

- Strong leadership from senior management in developing a vision and implementing a long-term strategy for world-class performance in the. management of hazardous materials.

- Extensive consultation and communication with employees to develop a shared understanding and commitment to the goals, strategies and procedures associated with the use of hazardous materials.

- A focus on safe production. 
- Better use of existing controls, together with adaptation and adoption of more advanced control technology.

- Deployment of training and awareness programs to enhance the skills and knowledge of management and employees.

- Commitment from employees via involvement and empowerment.

- Integration of efforts to cover the life cycle of chemicals (from manufacture to disposal).

- A culture of continuous improvement that permeates the organisation.

- Integrated approach to the management of hazardous materials - to link the efforts of the various departments that play a role in this, together with supplier and customer groups.

- Less hierarchical and less compartmentalised approaches, with greater flexibility.

As mentioned earlier, Bottomley (1994, p. 1) has put forward a framework for best practice in OHS. He suggests that there are three elements, all of which are necessary to ensure that continuous improvement is achieved in the medium to long term. The first element is the culture of the organisation. A crucial factor in creating best practice in OHS is the commitment of senior management and communication of this commitment to all levels in the organisation. Secondly, the organisation's management systems (the "software") need to be geared to the practical and systematic implementation and maintenance of the OHS culture. This software includes policies, standards, procedures, supervisory arrangements, training and similar. Thirdly, the physical components of the organisation's working environment (the "hardware") need to be purchased and installed with OHS considerations in mind.

Merry (1998, p. 15), in describing a method for assessing the safety culture of an organisation, refers to eleven characteristics of world-class safety performance. These are:

- Visible leadership and commitment from top management.

- Safety role of line management.

- Strategic business importance of safety.

- Supportive organísational culture. 
- Involvement of all employees.

- Organisational learning.

- Measurement of safety performance.

- Mutual trust and confidence between management and workforce.

- Openness of communications.

- Absence of the safety versus production conflict.

- Demonstration of care for all those affected by the business.

2.5.2 Performance measurement - concepts, processes and tools Strobach $(1990$, p. 42) observes that "the heart of management control is measuring performance in quantitative, objective terms. Too often, safety and health is not measured in this way."

According to Lindsay $(1992, p .390)$, "Health and safety performance in successful organisations is measured against pre-determined standards. This reveals when and where corrective action is needed to improve performance." This is a widely held view. For example, Fulwiler (1998, p. 27) claims that: "one of the essential characteristics of an effective management system is measurable outputs that lead to predictable results."

Gray-Spence (1994, p. 6) has outlined the rationale behind the measurement of OHS performance. In brief, performance measurement:

- Determines how well an organisation is performing.

- Determines the reasons for specific successes or failures.

- Reflects the effort involved in achieving organisational goals and objectives.

- Promotes an organisation's values.

- Provides the basis for program planning and development.

- Provides the justification for continued funding.

Crosby's research (cited in Sweeney, 1992, p. 91) put it that:

- The mere knowledge that performance is to be measured improves performance,

- The feedback from measuring performance leads to improvement, and 
- The communication of measurement of performance tends to improve performance.

Amis and Booth (1992, p. 45) expressed this in simple terms by borrowing from the words of management expert, Paul Drucker - "What gets measured gets done". The same authors have noted that performance measures should be designed to permeate every activity and function within the organisation, from top management decisions to shop floor behaviour (Amis and Booth, 1992, p. 45).

Meyer (1994, p. 96) suggests that a measurement system is not only the measures, but also the way they are used.

On the matter of measurement, Watson $(1992$, p. 82) notes the words of W. Edwards Deming that "arbitrary numerical goals do not, of themselves, breed quality performance. The key to implementation of improvements is the discovery of process enablers."

Johnson (1991, p. 11) identifies three broad groups of metrics applicable to continuous improvement. These are:

- Quality-related (measures of suitability and absence of defects).

- Productivity-related (output per unit of resource consumption).

- Timeliness-related (cycle time, time-to-market, and similar).

He states, firstly, that these measures should be applied at all levels in the organisation. Secondly, the measurement data must be capable of upwards summation and downwards differentiation. Thirdly, the data needs to be easy to collect (Johnson, 1991, p. 11).

Metrics for health and safety are sometimes described as leading or lagging, positive or negative performance measures, or as process or outcome measures (Alcoa, 1995, p. 9-1).

The UK Health and Safety Executive prefers the terms "active" and "reactive". It refers to active systems that are applied to monitoring the degree to which plans are fulfilled and the extent of compliance with standards. It then refers to reactive systems that monitor accidents, ill 
health and incidents (HSE, 1992, p. 47). Thus, the overall effectiveness of policy implementation is assessed by regard to:

- The degree of compliance with OHS standards.

- Identification of areas where standards are inadequate or absent.

- Achievement of specific objectives.

- Accident/incident data, together with information on immediate and underlying causes, trends and common features.

(Health and Safety Executive, 1992, p. 63)

Geller (1997a, p. 2) points out that outcome measures can be influenced by numerous factors, such as punishment and reward programs that can lead to under-reporting of injuries or near misses.

Traditionally, safety performance has been measured in reactive terms. Typical measures have included lost time injury rates (based on lost time injuries, duration one shift or more, per million hours worked), duration rates (the average time lost due to accidents), incident rates (near misses, damage or other loss events) and workers' compensation data (Australian and New Zealand Minerals and Energy Ministerial Council, 1999, p. 18).

Industry-wide safety performance reports tend to dwell almost exclusively on reactive or outcome measures. For example, the 2000/2001 Western Australian Minerals Industry accident and injury statistics are represented predominantly in terms of incident rate, duration rate, frequency rate and injury index (Department of Minerals and Petroleum Resources, 2002).

Geller (1997a, p. 2) has commented, like many others, that the traditional reactive measures of safety, such as lost workday rates, injury frequency rates and similar, have no diagnostic value to help understand or change system variables that produce outcomes. They reflect history and as a result are reactive and after-the-fact (Strobach, 1990, p. 42). They provide no facts about why an undesired event occurred (Arnold, 1992, p. 46). Amis and Booth (1992, p. 44) put it that "accident data is a surprisingly poor and incomplete measure of safety and health performance." Dial (1992, p. 41) was blunt with his assessment that "when used as short-term performance indicators or as a feedback mechanism for making 
reciprocative adjustments to the safety process, however, such rates are typically counterproductive." Glendon and Booth (1995, p.564) go further to provide a compelling list of reasons under the headings: "insufficiently sensitive", "dubious accuracy", "after the event" and "ignore risk exposure". According to Stephan, "Measuring safety according to injury statistics limits evaluation to a reactive, outcome-oriented perspective. Safety can compete with productivity goals only if measured in achievement terms and when emphasis is placed on safety management processes" (Stephan, 2001, p. 244). Furthermore, he suggests that "this management system should continuously track safety accomplishments and display them to the entire workforce (Stephan, 2001, p. 245).

Krause and Hidley (1989, p. 21) comment that "accident frequency rates are at best an indirect performance indicator, and at worst they are actually destructive to real safety performance improvement."

Amis and Booth (1992, p. 44) have expressed the prevailing view in the safety literature in relation to the use of lost time injury frequency rates (LTIFRs). They note that accident data:

- Measure failure, not success.

- Are difficult to use in staff appraisal.

- Are subject to random fluctuations.

- Reflect the success, or otherwise, of safety measures taken some time ago.

- Do not measure the incidence of occupational disease where there is a long latency period.

- Measure injury severity, not necessarily the potential seriousness of the accident.

- May under-report (or over-report) injuries and may vary as a result of subtle differences in reporting criteria.

- Are particularly limited for assessing the future risk of high consequence, low probability accidents.

The reporting of safety incidents is an important element within the lagging indicator group. According to Herbert (1994, p. 5), one reason incident 
data are so important compared to injury data is that there is so much more of them - more data to analyse and more opportunities to gauge the quality of the organisational effort Pidgeon (1991, p. 137) refers to the incubation model of disasters and suggests that "often, near misses will differ from actual disasters only by the absence of the final trigger event and the intervention of chance". He adds that "near-miss incidents can often be interpreted, not just with the benefit of hindsight, as warning signals (Pidgeon, 1991, p. 137). Reason (2000, p. 41) refers to these as "free lessons" and an important indicator of a "flexible safety culture" in which there is "a desire to learn and to constructively use new local information and insights."

It should be noted that incident reporting may be negatively impacted by management practices such as over-emphasis on incident-free records, punitive measures taken against injured workers, post-incident drug testing and making an example of an injured worker in front of co-workers (Roughton \& West, 2000, p. 27).

According to Ojanen, Seppala and Aaltonen (1988, p. 95), "Safety may, can, and should be analysed on various behavioural levels." They refer to management goals and strategies, the actions and performance of workers, the behaviour of workers, and accidents as areas to which attention should be applied.

Although Krause, Hidley and Hodson (1991, p. 51) see accident prevention as being best pursued by sampling the mass of safety-related behaviours which lie upstream of potential incidents, there is more to be measured than this.

Along these lines, Ragan (1997, p. 26) warns that behavioural safety is not the "holy grail" or "silver bullet" needed to achieve zero accidents - as promoted by some practitioners. He points out that "hazards cannot be aciequately controlled with good intentions and rigorous behavioural control" and "it does not matter how well safeguards and procedures are followed if they are based on flawed understanding of chemical processes or safeguard designs" (Ragan, 1997, p. 26). His concern is that when 
behaviour is measured, only behaviour changes, not the underlying belief system. In contrast, when the belief system changes, behaviour will follow (Ragan, 1997, p. 28). He advocates the use of perception surveys and other means to measure what people think is expected of them. Over time, employees will gravitate towards the actions and beliefs the organisation expects of them.

Other leading figures in safety research, such as Bailey and Peterson (1989), are strong advocates of the use of perception surveys to address safety system effectiveness. Further support for this approach comes from the Department of Minerals and Energy of Westem Australia (1996). The Department asserts: "Perception surveys can efficiently reveal the safety culture of an organisation." A series of questions can be developed to fall under the following principal headings:

- Leadership - management's demonstrated commitment to safety.

- Education and knowledge.

- Quality of the safety supervisory process.

- Employee involvement and commitment.

(Department of Minerals and Energy, 1996, p. 8)

Dial (1992, p. 40) has reviewed the downside of incident-focused management. He advocates use of upstream, qualitative indicators to measure progress and warn of potential problems. He suggests a role for questions such as:

- Is there a well-defined vision for the organisation? is it understood, shared and committed to by all employees (as verified through perception surveys)?

- Are the management systems continually evolving? Are weaknesses found during systern audits being corrected?

- Is there a long-range training plan, does it address all training-related deficiencies and is it quality assessed?

- Are critical behaviours affecting safety being identified and is there a process for determining and addressing the environmental factors influencing these behaviours? 
The National Safety Council (cited in Environmental Manager, 1992, p. 9) has noted that accident and injury records measure the number of such events, but provide no indication of program effectiveness. The NSC makes available three types of employee survey forms to assist companies to gauge the effectiveness of their safety programs. These are firstly, the Safety Barometer that assesses overall program health; secondly, the Occupational Safety Climate Assessment Report (OSCAR) that assesses the visibility of the safety program, employee involvement and perceived effectiveness of the program. Thirdly, there is the Business and Safety integration Survey (BASIS). This assesses collective values and norms that guide the management team and how they operate in the management system. It also highlights inconsistencies between business and safety management at each organisational levei and provides recommendations for improvement in the context of the company's total management style (Environmental Manager, 1992, p. 9).

The matter of employee perception of effectiveness appears to be a key indicator of the health of the programi.

Over the last several years, positive performance indicators have become popular. These monitor current conditions and activities, such as design, development and installation of management and workplace initiatives that seek to prevent incidents and improve health in the workplace (Griffiths, 2000 , p. 3). Accurding to Merry (1998, p. 18), the innovative use of leading indicators to identify latent weaknesses allows the organisation to take early remedial action to correct weaknesses and avoid degradation in safety performance.

Quinlan (1999, p. 20) offers examples of positive performance measures monitoring of the extent of use of personal protective equipment, the extent to which employees identify and report hazards, the level of participation in training, and similar.

Griffiths suggests that the scope for measurement covers all engineering, organisational, procedural, behavioural and personal protective equipment controls. Thus, measures may be applied to audit systems, job and task 
observations, inspections, the time taken to complete corrective actions, quality of audits, maintenance and other safety-related activities (Griffiths, 2000, p. 4).

Thus, metrics for health and safety come in two forms - variously described as leading or lagging indicators, positive or negative indicators, or as process or outcome measures.

The Australian and New Zealand Minerals and Energy Ministerial Council (1999, p. 19) has suggested the following as areas to consider for performance measurement activity:

- Attitude surveys

- Achievement of objectives and implementation of plans

- Deployment and understanding of procedures and risk management tools

- Compliance with standards (including legal obligations)

- Processing of audit corrective actions

- Competence verification (measurement of learning outcomes)

- Observable behaviour during work activities

- Integrity of plant and equipment (via checking of maintenance, testing and inspection routines, equipment failure rates and similar)

- Effectiveness of health hazard controls (via measurement of the workplace environment and monitoring of employee health)

- Emergency response (meeting targets and schedules for emergency response drills).

It was noted earlier that modern safety literature suggests that most accident and ill-health outcomes are related to management failure. This raises the question of what measures should be applied to management's input into the programs, systems and processes that exist in the organisation. Strobach (1990, p. 42) provides a few examples and suggests that quality and quantity aspects should be examined for each of these:

- Induction training

- Inspections 
- Task observations

- Rule compliance

- Group meetings

- Personal protective equipment usage

- Personal communications

- Promotional efforts

Herbert $(1994$, p. 8$)$, in reflecting on the DuPont approach, identifies the management audit program as one of the most important leading indicators of safety performance. By this, he refers to managers walking around the site, talking with workers, encouraging safe behaviours, communicating standards, building relationships and so forth. Elsewhere this approach is referred to as management safety contacts and is embedded in implementation plans (Alcoa, 2001b, p. 40, Alcoa, 2002b, p. 7). The activity is readily measured and is a suitable element in performance expectations for line management.

Nedved has reviewed the development of positive performance indicators for use within the mining and mineral processing industries of Western Australia. He concludes that such indicators should be fully utilised in the development of occupational health and safety management systems (Nedved, 2000, p. 10).

According to Brandt (1997, p. 28), in selecting the best performance measures, the following characteristics need to be considered:

- The behaviour that needs to be influenced to achieve the goals

- Alignment with the goals

- Simplicity

- Quantifiable

- Focused on results

- Ease uf collection.

Performance measures for the job observation component of behavioural safety might take the form of:

- Number of observations per month 
- Percentage of work groups participating per month

- Percentage of safe behaviours

- Percentage of at-risk behaviours

- Comparison of the percentage of safe behaviours to the injury frequency rate.

So, are there macro predictors of safety performance? According to Amis and Booth (1992, p. 46) the answer is "yes". They identify the following as useful predictors of performance:

- The time people spend in the organisation thinking about safety (organisational focus)

- The effectiveness of the organisation's system of two-way communications (shared perceptions of goals)

- The capacity of the organisation to identify the need for, and respond to, change (with good organisational learning attributes), and,

- The financial climate of the parent organisation (or the organisational climate within which the company is working).

The same authors provide a checklist of factors to apply in judging the suitability and comprehensiveness of a battery of health and safety performance indicators:

- Is the indicator measuring the implementation of programs, or the effectiveness of the program?

- Is the indicator a direct indicator of performance (a lagging indicator) or a predictor (a leading indicator) of performance?

- Is there a reasonable balance between subjective and objective information, and qualitative and quantitative data?

(Amis \& Booth, 1992, p. 45)

Although there have been many authors who have pointed out the shortcomings of the outcome measures, few have said they don't have any place at all. Mitchell (1993a, p. 3) puts it that "safety statistics can be used effectively to track progress over time if the limsits of random variation are defined and their significance understood". According to Mitchell (2000, p. 319), "a suitable mix of performance indicators ... outcome- 
oriented and positive-oriented ... is needed to provide a comprehensive view of OHS performance. Sweeney (1994, p. 38) suggests that "there is no escaping LTIs, nor should there be ... it is a combination of LTIs and positive performance indicators that will, in the end, have the biggest impact on reducing lost time injuries."

Mitchell $(2000$, p. 322$)$ notes that the maturity of the OHS management system within the organisation will determine what performance indicators are suitable for use.

The National Industry Extension Service (NIES) points to the importance of understanding the critical success factors of the business and the associated business processes. This puts one in a much-improved position to decide which indicators to use for performance measurement, and the changes that may be needed to achieve desirable outcomes (National Industry Extension Service, 1999, p. 12).

In summary, there are numerous leading and lagging performance measures that can be applied in the OHS area. The objectives of the monitoring effort are not only to determine the immediate causes of the sub-standard performance, but, more importantly, to identify the underlying causes and the implications for the design and operations of OHS management systems (Lindsay, 1992, p. 390). Without performance measurement systems, it is impossible to determine how well the continuous improvement process is being implemented (Sweeney, 1992, p. 90). Finally, the words of Dial $(1992$, p. 43) sum up the widely held view that "a vision to create a continuous improvement environment steers efforts where they are most effective - upstream in the organisational culture and management systems."

\subsubsection{Performance Reporting and Review}

In a modern workplace setting there is a need for business performance data to be expressed in clear and unequivocal terms, and in a form that can be readily communicated to stakeholders. OHS data is no exception to this. There are many ways to report and review performance and 
progress in OHS. Three new approaches that have had an impact in recent years are safety dashboards (first described by Meyer), the safety performance index approach (developed by the Eastman Kodak organisation) and the balanced scorecard approach (developed by Kaplan and Norton). These may be summarised as follows.

Christopher Meyer asserts that performance measurement systems are an essential support for multifunctional teams. A team's measurement system should primarily be a tool for telling the leam when it must take corrective action (Meyer, 1994, p. 96). He suggests that companies may find it helpful to create a computerised "dashboard". This approach can support a team of co-workers in the same way that a dashboard in a car provides the driver with essential information on the journey at hand. "The dashboard format, complete with colourful graphic indicators and other easy-to-read gauges, makes it much easier for a team to monitor its progress and know when it must change direction" (Meyer, 1994, p. 98).

This concept has been applied in a refinery setting in Western Australia and is ciescribed by Calder and Davies (1996). They point out that the dashboard approach provides an opportunity to display a number of performance indicators on one page. In their system, indicators are rotated each week - to give the dashboard a fresh look and to allow tracking of a large number of indicators (Calder \& Davies, 1996, p. 6). They explain that indicators should be chosen on the basis of their ease of measurement and their relationship to the health and safety effort. Ideal rneasures are those that are part of existing databases and therefore absorb little time in getting them ready for presentation (Calder \& Davies, 1996, p. 7).

They identify five key benefits that have arisen from use of the dashboard approach:

- Increased focus and assistance in directing activities and resources to problem areas.

- Improved workforce knowledge of performance in the processes. 
- Intemal benchmarking opportunities (between site groups/departments using the tool).

- Facilitates deployment of a wide range of performance indicators.

- Facilitates efficient tracking to completion of activities.

(Calder \& Davies, 1996, p. 7)

Another tool for reporting safety and health performance is the Safety Performance Index, originally developed by the Eastman Kodak organisation. This model requires the selection of indicators (generally no more than seven), the assignment of a weighting factor to each, to a total of $100 \%$, and the esiablishment of a baseline (current performance), target performance and ideal performance for each indicator. A score can be derived for each indicator and an overall performance index can be obtained (Eastman Kodak, 1994, Calder \& Davies, 1996, p. 8).

The balanced scorecard concept, as developed by Kaplan and Norton of the Harvard Business School, has emerged as an important tool for measuring and reporting busiriess performance. It calls for the use of a wide range of positive performance indicators, thereby opening the door for OHS-based indicators to be brought into the mainstream of management (Sweeney, 1994, p. 43). Most balanced scorecards cover goals and objectives, key performance indicators, a description of the present state, a description of the future (desired) state, together with action items, responsibilities and timelines. They will:

- Promote a well-balanced business strategy with seamless integration of critical success factors.

- Enable the translation of strategic objectives at the enterprise level into coherent deployment plans and progress measures.

(Baldridgeplus.com, 1999, p. 4)

Kaplan and Norton (cited in Sweeney, 1994, p. 43) consider that the benefits of a balanced scorecard include:

- Making strategy operational by translating strategy into performance measures and targets. 
- Helping focus the entire organisation on what must be done to create breakthrough performance.

- Acting as an integrating device for a variety of often-disconnected corporate programs.

- Facilitating vertical and horizontal alignment of efforts with the strategic objectives of the organisation.

- Helping to break down corporate level measures, so that all can see what they must do to improve organisational effectiveness.

Some samples of an OHS dashboard, a performance index and a balanced scorecard are provided in Appendix 3.

\subsubsection{Audits - concepts, processes and tools}

Modern legislative instruments, such as the Mines Safety and Inspection Act 1994, require employers to provide and secure safe systems of work. This implies that it is necessary for the employer to check the adequacy and continued effectiveness of arrangements to prevent adverse health and safety outcomes at work. Audits have been a key device in carrying out this monitoring function (Williams, 1993).

According to Waterhouse $(1992$, p. 15), safety auditing had its origins in the early sixties. The landmark Report of the Robens Committee 1972, referred to eartier, described safety audits as a management diagnostic and predictive technique (cited in Waterhouse, 1992, p. 15).

OHS auditing supports the measurement effort described above by providing information on the implementation and effectiveness of plans and performance standards. It also provides a check on the reliability, efficiency and effectiveness of the arrangements for policy-making, planning, implementing, measuring and reviewing performance (Lindsay, 1992, p. 398).

There are numerous definitions of auditing. Most talk in terms of an organised review and reporting process that uses interviews, plant inspections and record reviews to develop an accurate picture of periormance at the facility (Glendon, 1995, p. 570). Lindsay (1992, p. 
$387)$ notes that audits need to be systematic, methodical and scientific. Waterhouse (1992, p. 15) refers to an "independent examination of the arrangements made by the employer for the achievement of satisfactory health and safety standards at the workplace, and other places affected by work activities."

The audit provides the location with an independent assessment of progress in various areas. It offers a systematic and structured framework for verifying that what is being done conforms with what was planned (Bottomley, 1994, p. 10). Glendon (1995, p. 572) put it that the OHS audit is a tool for improving both the efficiency (doing things right) and the effectiveness (doing the right things) of management performance in designated areas.

Recommendations for change or continuance of the strategy are the main outcomes of an audit (Waterhouse, 1992, p. 15). Done regularly, they will ensure that activities are aligned and that the organisation's vision, planning functions and actions are integrated vertically and horizontally (Cook \& Blaxter, 1991, p. 151). In the words of Lindsay (1992, p. 398), "Auditing supports monitoring by providing managers with information on the implementation and effectiveness of plans and performance standards. It also provides a check on the reliability, efficiency and effectiveness of the arrangements for policy-making, planning, implementing, measuring and reviewing performance."

Thus, audits create a learning opportunity and will help to identify strengths and opportunities for improvement. Furthermore, they will assist in identifying best practices and in facilitating the transfer of health and safety technology.

Arnold (1992, p. 49) identifies eight key benefits of a safety audit system:

- It offers a precise evaluation of an organisation's safety performance.

- It provides a means for appraising individual and group safety performance.

- It serves as a guide for implementing a modern safety and health management program. 
- It acts as a practical, on-going means for identifying the majority of safety, health and environmental loss exposures facing an organisation.

- It unequivocally indicates that management cares about employees.

- It offers an opportunity to educate and involve line management in the organisation's safety and health program.

- It establishes the organisation's capability to forecast the potential for loss-producing events.

- It heips reduce operating costs by eliminating management inefficiencies that lead to accidents and other losses.

The audit can be applied to the whole organisation or simply to a department (Dare, 1995, p. 3). It can be directed at management systems such as leadership, commitment, organisation for safety and change management, or may be directed at more specific topics such as radiation protection, hearing conservation, falls protection, confined space safety and similar.

Auditing tools and processes have relevance to the wider industrial community. In Western Australia, the Department of Minerals and Energy uses a locally developed audit guideline document as the basis for evaluating OHS management systems at mine sites that fall under its jurisdiction (Department of Minerals and Energy, 1997b).

There sre many questions to be resolved in setting up an auditing prograti. These include:

- What activities/subjects should be audited (the facility, a department, a process or a system (such as a permit to work system)?

- Who should be involved in the conduct of the audit?

- Who is to select the subject of the audit?

- Within that subject, what factors should be examined?

- Against what criteria should factors be compared?

- Should factors be given equal or variable weight? 
- For multi-site operations, should differences (eg, in age of the plant, different raw materials, processes and similar) be taken into account in determining a score?

- Should the audit be a numerical or a subjective assessment, or a combination of the two?

- Should the audit package be an off-the-shelf product or something developed in-house?

- What information should be provided by the area/department under survey, prior to the audit?

- What form should the report take?

(Alcoa, 1999c, p. 40)

Wallace (1991, p. 19) notes that some audits degenerate into housekeeping inspections, with negative results. He cautions that lack of time, and pressure to look at everything, may mean that nothing is looked at in-depth and that only a superficial appreciation can be gleaned for most elements.

Nedved (1999a, 1999b, 1999c, 2000) has reviewed safety audit practices within the mining and mineral processing industries. He has examined areas such as the development of audit protocols, the development of selfaudit systems and the development of positive safety performance indicators.

Calder (1996) has described how a formal corporate audit within a major global organisation, Alcoa, has been adapted and simplified for use at a departmental level within a location. The purpose was to provide departments with a practical tool to measure their performance and provide knowledge and experience on how to continuously improve their OHS processes. Since the Company's international audit protocols are quite complex and were developed for use by trained auditors, it was necessary to modify these to enable them to be deployed by inexperienced auditors within the general workforce. They were developed into audit work sheets that systematically moved through the audit process in a way that would enable a novice auditor to arrive at an audit rating for 
the particular element and to make recommendations for improvement within the department.

Calder describes this self-assessment activity as a five-step process - as represented in Figure 7 below.

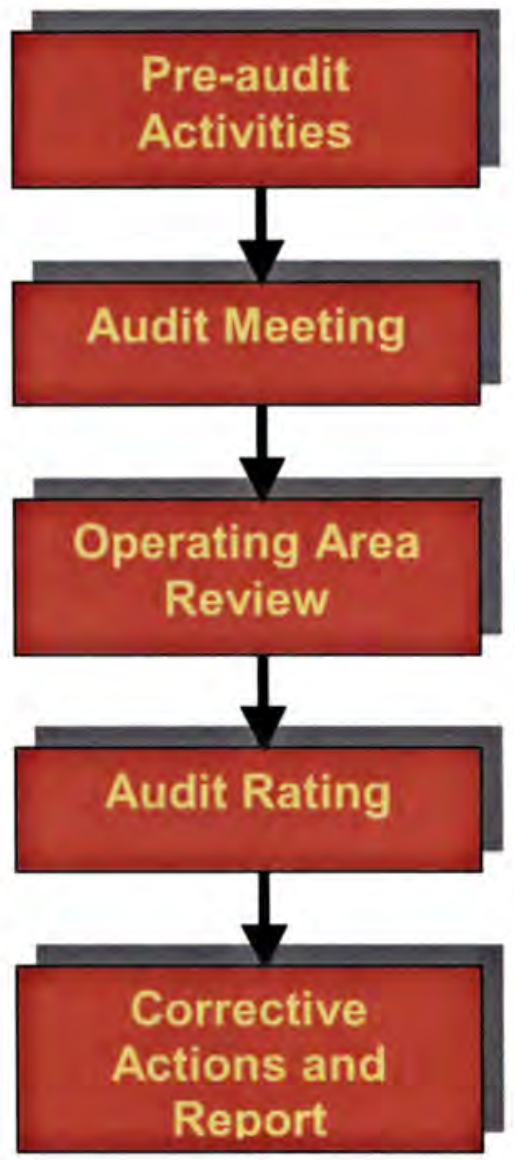

Figure 7: Self-Assessment Audit Process

(Calder, 1996, p. 5)

Pre-audit activities include selecting the audit team and distributing the designated audit protocol working papers to the team members. It may be necessary for the team leader to convene a meeting of the team in advance of the audit - to explain the purpose of the audit and links to other activities. Members are expected to review and complete the working papers prior to the audit meeting. 
At the audit meeting, the team reviews and responds to the discussion questions that have been provided. This collective response is then subject to verification in the field.

The operating area review involves physical examination of the work environment and checking with other employees in regard to their understanding and use of the various initiatives, controls, procedures and similar that have been identified at the audit meeting. The audit protocol working papers establish the observations that should be made and the questions to ask to verify that such measures are in place (Calder, 1996, p. 7).

The fourth step involves the team deciding on a performance rating for the particular elements being scrutinised. Criteria are available to assist this process.

Finally, the team makes a series of recommendations for improved performance. Findings become a record for future reference in subsequent audits. The Department is then responsible for managing the scheduling of future audits, tracking the completion of audits, checking on the response to recommendations and reporting the performance of the department to other relevant groups (Calder, 1996, p.10).

Self-assessments of this kind deliver a number of benefits. They have potential to:

- Promote a stronger internal control environment

- Facilitate the management of business risks

- Identify potential problems and process improvement opportunities

- Enable a wide cross-section of participation and thereby encourage understanding and ownership of business processes deep within the location or business unit

- Facilitate the audit planning and risk assessment processes

- Reduce process variability

- Provide cross-training opportunities

- Facilitate the identification and sinaring of exceptional practice 
- May reduce the depth, extent or frequency of formal corporate audits. (Alcoa, 1999b, p. 30).

Self-audits have become a powerful tool in the search for best practice. Alcoa has an on-line auditing system designed to help operating facitities and individual work groups continuously improve their environment, health and safety performance (Alcoa, 1999a, p. 37).

Alcoa recently surveyed its business units and locations throughout North America, Europe, Australia, Asia and Latin America to establish viev/s on the usefulness or otherwise of its self-audit processes. Among key findings were that more than 85 percent of respondents believe that performing a site self-assessment leads to the improvement of a business unit or location's processes or increases its control over business risks (Alcoa, 2000, p. 3).

The report which followed the investigation into the 1988 King's Cross disaster in the U.K. gave a ringing endorsement of safety audits as a monitoring tool. Desmond Fennell, QC, (cited in Deacon, 1994, p. 19) said: "If the internal audit has become the yard stick by which financial performance is measured, then the safety audit should become the yard stick by which safety is measured."

According to Lindsay (1992, p.402), the organisational culture in which the audit is applied is as important as the detail of question sets or scoring systems.

Alcoa (1999b, p. 45) has described a broad process for auditing and this is represented in Figure 8. 


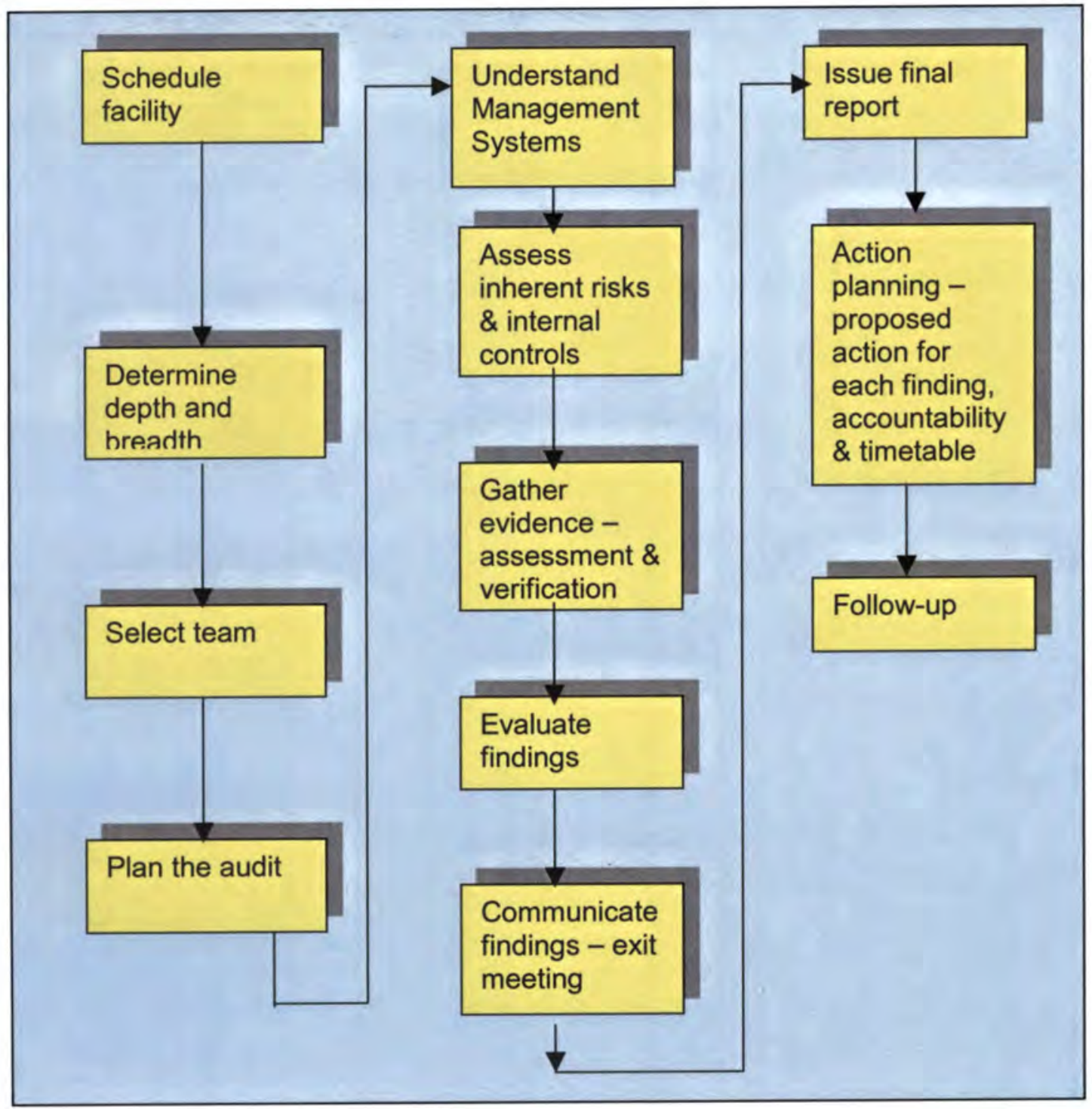

Figure 8: The broader audit process

(from Alcoa, 1999b, p. 45)

Alcoa (1999c, p. 36) nominates three common methods for collecting audit evidence, namely:

- Inquiry. This involves formal and informal questioning

- Observation. Physical examination of the workplace is usually very reliable. However, there are some limitations. For example, the presence of control equipment, monitoring devices, alarms and similar does not mean they are appropriate for the task at hand, that they are functioning correctly or that they will continue to function correctly. 
- Verification testing. This covers retracing data (beginning from the originat document and tracking forward), re-computation (reworking the calculations), vouching (beginning vith the record and working backwards to the original document) and confirmation (obtaining written evidence from independent third parties). Employee interviews play an important part in verification. As Amold (1992, p. 48) put it, "to determine how well a system is functioning, an auditor should ask those people in the best position to provide honest answers: employees."

Arnold (1992, p. 48) calls for the audit to examine a program's "vital signs" and states that measurements need not be complex. He advocates measures and indicators such as percentages of inspections completed, investigations completed, permits properly used, employee compliance with personal protective equipment requirements and required group meetings held. Used appropriately, the measures reflect the efficacy of critical safety and health efforts at the facility.

Moreover, he makes the point that auditors often struggle to find ways to measure quality. He claims that this need not be the case and that auditors should simply measure compliance to established criteria. A value factor should be applied to each criterion reflecting its relative importance to achieving the desired result - and it is then a matter of establishing whether criteria have been met. This process may be applied to measurement of any program activity: investigations, procedures, employee training, group meetings, and similar (Arnold, 1992, p. 48).

Audits provide a snapshot of health and safety management system performance. Many audits are associated with a scoring arrangement and this provides a way of measuring progress with individual elements or with broader programs. Audit protocols are used to guide the auditor during the assessment and a ratings system can represent progress in a meaningful way (Nedved, 1999b, p. 8). Alcoa (1999b, p. 39) uses a fourlevel rating system with criteria to accompany each level. The description "Excellent" is applied to state-of-the-art performance, "Good" to a solid 
effort with no program deficiencies, "Fair" to a situation where some program deficioncies exist and "Poor" where many program deficiencies exist and there is significant potential for compliance issues. The score that is obtained for an organisation, a department, a process or a system is arrived at after the auditors consider evidence collected during the audit. It is important to accept that every important detail can't be covered during the exercise. However, the number of ways information is validated will impact on the confidence that can be applied to the results and auditors will pursue this within the time constraints that are imposed (Alcoa, 1999c, p. 36).

Amold (1992, p. 49) advises that: "Ideally, an auditor is not a fault-finder, but a fact-finder. If audit results fail to recognise and commend the positive, a tremendous opportunity for ensuring continued positive behaviour is lost."

The Chamber of Minerals and Energy of Western Australia (1993) describes a self-audit system it produced for its member companies. This was aimed at small-medium sized operations that needed a simple tool to determine the strength of their EHS management systems. The system has $2 i$ elements and these are subject to three forms of activity:

- Self-audit of the essential eiements of a comprehensive EHS program.

- Identification of areas where performance is below that which the user believes is appropriate and determination of priorities for remedial action.

- Development of a follow-up plan and identification of future targets.

(Chamber of Minerals and Energy of Westem Australia, 1993)

Shaw notes that system auditing is frequently advocated in the literature as an approach to monitoring and assessing OHS management systems. A large number of proprietary methodologies exist, particularly the International Safety Rating System and its derivatives such as the Five Star System of the National Safety Council of Australia (Shaw, 1994, p.18). Such systems have attracted criticism - mostly on the grounds of 
their complexity and the perceived lack of correlation between star ratings and reportable injury rates.

In 1994, the Department of Occupational Health, Safety and Welfare of Western Australia (DOHSWA) introduced a self-assessment package, the Jobsafe Flan. This aimed to promote the OHS management practices needed to establish and maintain systems of work to minimise employee exposure to hazards and reduce rates of lost time injury and disease (Department of Occupational Health, Safety and Welfare of Western Australia, 1994a, p.1). The assessment is divided into five key elements, namely management commistment, OHS policy, plans and procedures, consultation, hazard identification, risk assessment and control, and training. The preliminary self-assessment uses 29 of the Jobsafe Plan indicators, while there are 133 indicators in the full assessment used by the Department. Each indicator is given a score out of ten and the scores are averaged to provide an overall rating of performance in each element (Department of Occupational Health, Safety and Welfare of Western Australia, 1994a, p. 2).

The Victorian equivalent of the Jobsafe Plan is SafetyMAP (Safety Management Achievement Program). This offers a set of benchmarks and performance indicators that can be used to evaluate progress towards OHS best practice (Winder, Gardner \& Trethewy, 2001, p. 70). The SafetyMAP program has three core components, assessment, audit and achievement, and these are applied to 12 system elements. The latter all carry associated audit criteria that facilitate a comprehensive assessment of OHS management at the location. Many of the principles of quality management are incorporated in SafetyMAP. Additionally, the program is consistent with the thrust of performance-based legislation ano quality management trends (Bottomley, 1994, p. 6).

Alcoa $(2000$, p. 2) has described a series of elements that constitute a self-assessment effort. This is represented in Figure 9. 


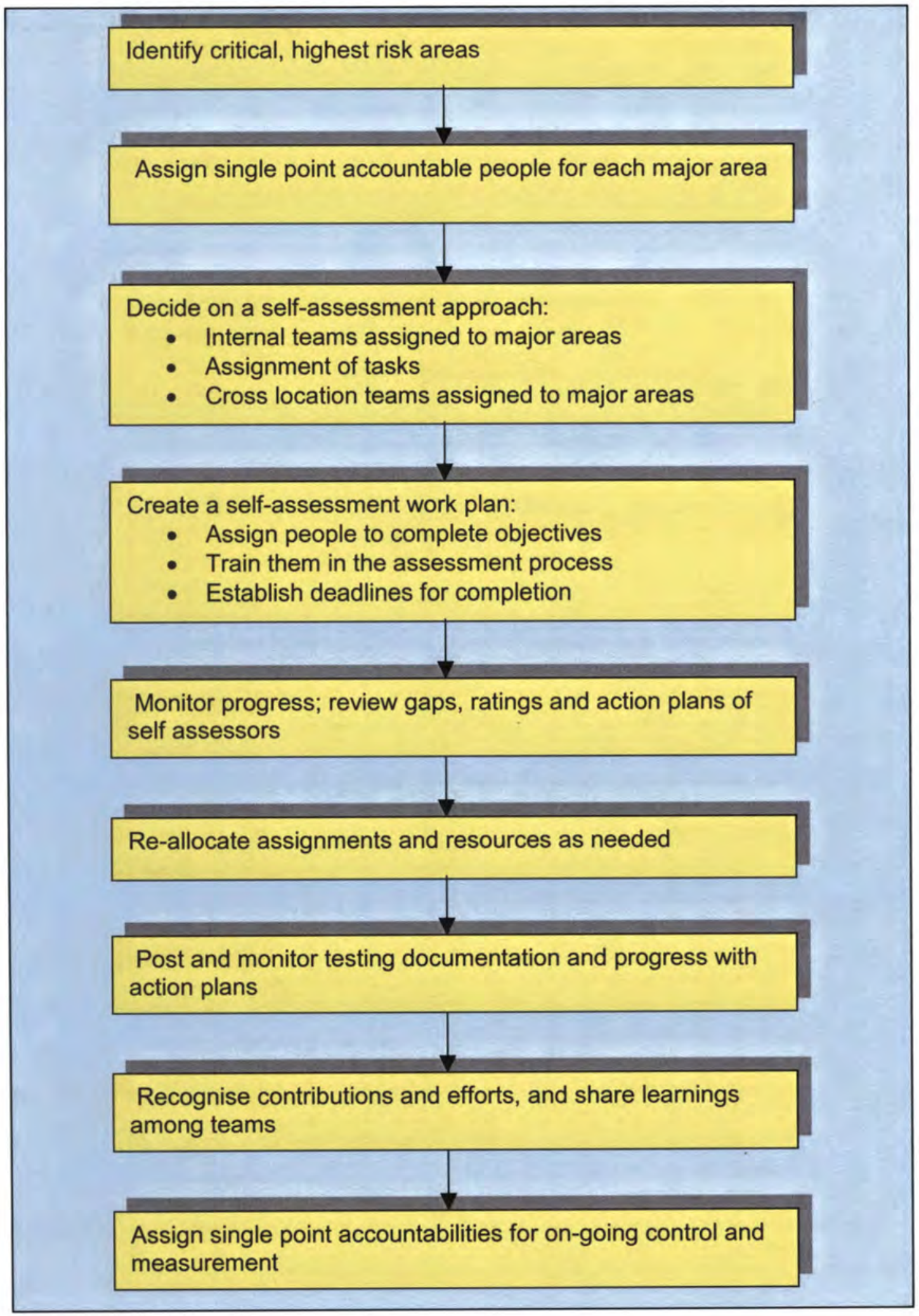

Figure 9: A flowchart for self-assessment deployment (adapted from Alcoa, 2000, p. 2) 
Kase and Wiese (cited in Glendon, 1995, p. 573) offer some advice on presentation of results at the end of an audit exercise. They suggest that the following approach be applied:

- Present both positive and negative findings.

- Cite sources and evidence in support of findings.

- Do not present problems for which solutions don't exist.

- Always offer solutions, recommendations or corrective actions.

- Where options exist, appeal to the manager's expertise to arrive at the optimum corrective action.

- Anticipate that time will be needed for evaluation and decision-making.

- Follow-up.

Auditing processes and tools are not the sole domain of private organisations. In Western Australia, the Department of Minerals and Petroleum Resources (now the Department of Industry and Resources) uses a locally developed audit guideline document as the basis for evaluating management systems in operations that fall under its jurisdiction - essentially, the mining and mineral processing operations of Western Australia.

The DMPR audit structure covers eight elements. There are 21 subelements and 146 individual points or standards beneath these. The principal headings are:

- Corporate and enterprise leadership.

- Safety plans to ensure continuous improvement.

- Occupational heaith programs.

- Safety support services.

- Risk assessment and hazard analysis.

- Work practices.

- Pre-employment processes, training, communications, behaviour and culture.

- Employee involvement and accountability.

(Department of Minerals and Petroleum Resources, 2002) 
Lindsay (1992, p. 402) points out that the full potential benefits of auditing will be seen only when the audit systems are applied vigorously and consistently. Furthermore, the findings need to be interpreted by skilled and informed people who will, in time, transmit these to the decisionmakers who have the power to initiate change and achieve progressive improvement.

\subsubsection{Benchmarking - concepts, processes and tools}

The Prussian military leader, Prince Otto von Bismarck (cited in Watson, 1992, p. 91), once said: "Fools you are ... to say you learn by your experiences ... I prefer to profit by others' mistakes and avoid the price of my own." This is a pragmatic approach that applies not only to the context of warfare, but also to the conduct of business and, for that matter, to life in general.

In the late 1970 s, the Xerox Corporation was facing severe financial and competitive pressures. It responded by developing and implementing a process called benchmarking. Within a few years it regained market share, drarnatically lowered costs, improved quality and avoided financial disaster (Pryor, 1989, p. 28). Initially, management's aim was to analyse unit production costs in manufacturing operations. However, the effort was so successful that the Company's leaders directed all units and cost centres in the corporation to use benchmarking as a key element of improvement efforts (National Industry Extension Service, 1999, p. 1). This created some difficulties, since the support functions found it difficult to arrive at a convenient analogue to a product (Tucker, Zivan \& Camp, 1987, p. 8). Eventually, these problems were overcome and benchmarking became what it is today - an extremely useful improvement tool for a wide range of activities within an organisation.

The term benchmark is defined in Webster's Dictionary as "a standard or point of reference in measuring or judging quality, value, etc" (Neufeldt \& Guralnik, 1988, p. 129). As indicated earlier, benchmarking, the activity, is an important component of the quality management approach. It offers the opportunity to fast track improvement opportunities. There are strong 
links between benchmarking, strategic plarining, continuous improvement (the TQM approach referred to earlier) and employee empowerment (National Industry Extension Service, 1999, p. 13).

Watson (1992, p. 119) sees benchmarking as "a basic business tool for management; it reinforces the other quality tools that are used for implementing continuous improvement in all of the business processes." According to the Health and Safety Executive, benchmarking is an important business improvement tool and can be applied to any area of activity, including OHS (Health and Safety Executive, 1999, p. 1).

In 1992, Watson produced a landmark benchmarking workbook. Apart from dealing with definitions and concepts, he provides sample worksheets for the identification and analysis of critical success factors, and a benchmarking study checklist. In addition, he provides templates for comparing business performance, doing benchmarking partner analysis, questionnaires, action plans, goal setting, implementation and the tracking of critical success factors (Watson, 1992).

In 1993, the National Industry Extension Service (NIES) published a comprehensive benchmarking self-help manual. In that, they describe benchmarking as a very powerful tool for organisational change and a means to strengthen all aspects of a business (National Industry Extension Service, 1993, p. 4)

Several years ago, the National Occupational Health and Safety Commission developed a benchmarking manual aimed at helping Australian enterprises pursue best practice in OHS. The package includes guidance for individuals working to establish OHS benchmarking within their organisations, as well as OHS committee members, OHS representatives and OHS managers (National Occupational Health and Safety Commission, 1996a; Blewett \& Shaw, 1995b, p. 237).

The United Kingdom Health and Safety Executive says that benchmarking is directed at learning from others, learning more about your organisation's 
strengths and weaknesses, and then acting on the lessons learned (Health and Safety Executive, 1999, p. 1).

Pryor (1989, p. 28) asserts that "simply stated, benchmarking is the comparison of a given business function across companies." Furthermore, he suggests that benchmarking involves:

- Measuring your performance against that of best-in-class companies

- Determining how the best-in-class achieve those performance levels, and

- Using the information as the basis for your own company's targets, strategies and implementation.

According to Bob Camp in his book Benchmarking (cited in Mitchell, 1993c, p. 17), benchmarking is a process which involves continually researching for new ideas and methods, processes and practices, and sither adopting or adapting the best features of these. It is important to not only identify what is best, but also to understand how the best is achieved. Camp (cited in Jay, 1994, p. 34) warns that it is important to focus on the steps and procedures that produce the "benchmark" outcome. He claims that, "Concentration on the benchmark, the measure, is really an empty statement until it is traced back to the practice - the best practice - in the process that achieved the performance."

Blewett and Shaw (1995b, p. 237) see benchmarking as "identifying an enterprise's needs, finding another enterprise which does it better, and learning from it." Furthermore, they note: "Benchmarking is about building up relationships and communicating ideas between enterprises. Watson (1992, p.5) uses a similar theme in defining benchmarking as a process of "measuring your company's method, process, procedure, product, and service performance against those companies that consistently distinguish themselves in that same category of performance."

According to Colin Mills from the Australian Quality Awards Foundation (cited in Jay, 1994, p. 34), "Benchmarking is an important process for fasttracking improvement activities." Furthermore, he points out that, "You do not have to carry out all the improvement work from your own resources. 
If the process is an important one and you wish to improve it rapidly, find out how other organisations do it and learn from them" (Mills, cited in Jay, 1994, p. 34).

Lindsay (1992, p. 401) sees benchmarking as a means of comparing management practices, techniques and outcome indicators, such as accident rates, with those of organisations in the same line of business. The purpose is to provide a wide perspective and gain new insights on the management of similar problems.

Benchmarking is a way of examining best practice and learning from it. Information generated is used to establish requirements, develop priorities, plan strategies, and implement process changes. Knowledge gained can help identify improvement opportunities, eliminate unnecessary processes, and create new products and services (Pryor, 1989, p. 29). As Alcoa (1990, p.6) put it, "To realise value from benchmarking, the information must be utilised in strategic decisions or in quality improvement actions."

The National Industry Extension Service $(1993$, p. 8) identifies several complementary objectives or benefits attached to benchmarking. These are:

- Assists identification, understanding and implementation of best practice in the area of interest.

- Helps to overcome complacency and inertia, and tends to emphasise the need for change.

- Helps to build and reinforce commitment to change.

- Creates an opportunity to achieve a substantial lift in performance.

- Assists in developing a shared vision within the organisation.

There are different types of benchmarking. The National Industry Extension Service (1993, p. 9) identifies fundamental distinctions between internal and external efforts, and whether the main focus is upon numbers or processes. Intemal benchmarking involves comparisons between departments, facilities or business units within the same organisation. External efforts may be carried out with competitors, others within the 
same industry grouping, or organisations that are part of completely different industries. Sometimes, benchmarking is focused on performance indicators (numbers), such as accident rates, workers' compensation costs, service or product delivery costs, and similar. Altematively, it may focus on the business processes that drive performance indicators. Examples in the OHS area would be arrangements for procurement and vetting of hazardous materials, training delivery or processes for the development of rules and procedures (National Industry Extension Service, 1993, p. 9).

Figure 10 below represents a generic benchmarking process, as depicted by Shaw (1994, p. 27).

Partner selection is an important element in the benchmarking process. The Health and Safety Executive (1999, p. 6) outlines advantages and disadvantages of internal and external benchmarking. For benchmarking within the same industry, they offer three key advantages (and no disadvantages):

- Quite easy to identify potential partners.

- Links are often already in place.

- It is likely that they "speak the same language" and share issues and concerns.

Tucker, Zivan and Camp (1987, p. 9) note that the Xerox Corporation relies strongly on presentations at professional and other forums, trade journals, consultants, annual reports and other company publications in which "statements of pride" appear as a means of identifying superior performance. They observe that: "the same well-run organisations keep turning up." They comment that cooperation at the professional level is not difficult to obtain since such individuals are usually eager to compare performance within their functional area with that of others. 


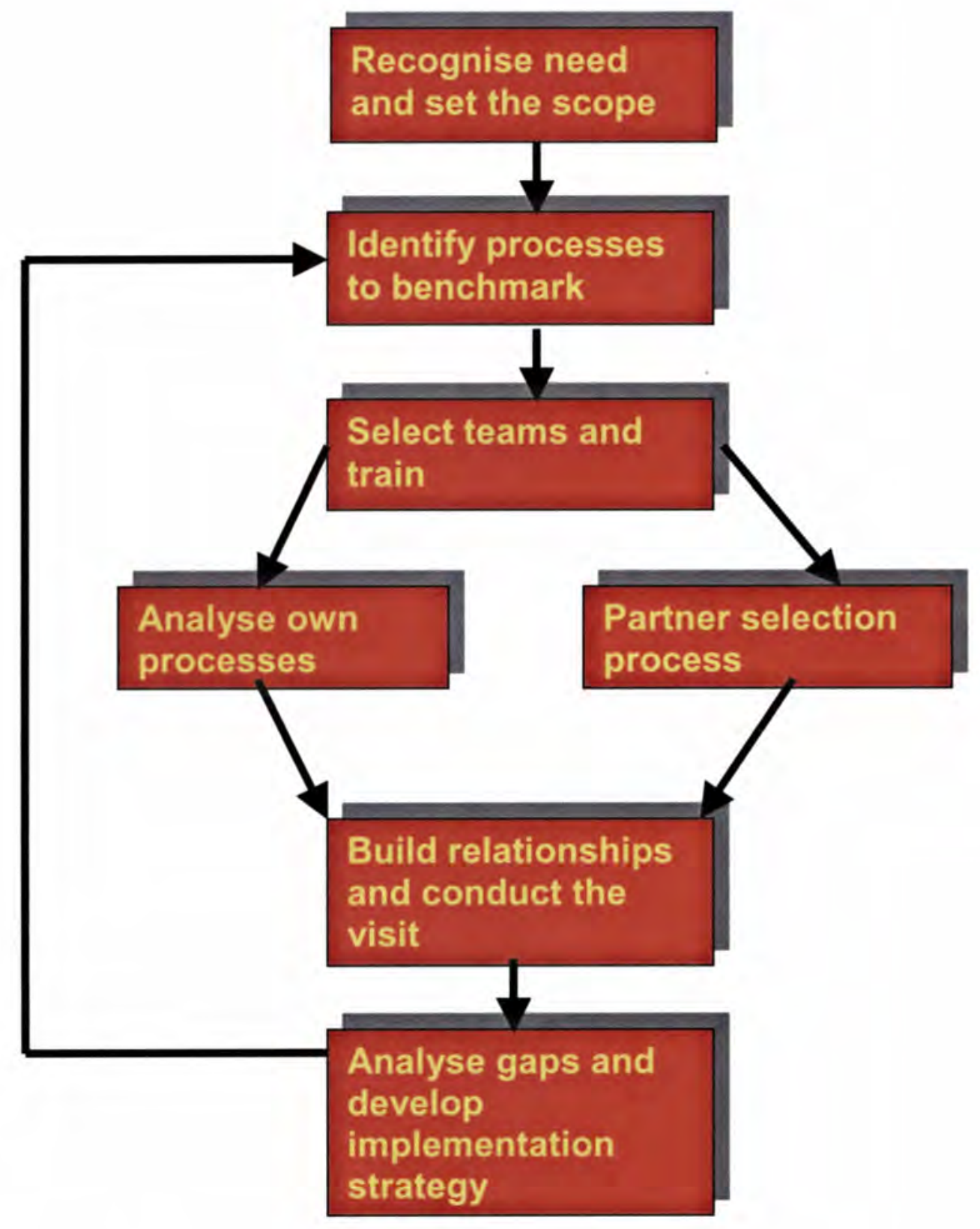

Figure 10: A generic benchmarking process

(from Shaw, 1994, p. 27)

Benchmarking contains a self-study step - to produce a baseline of quantified information on products, processes and services that can be used to compare with equivalents within the operations of the benchmarking partner. This is a valuable exercise in its own right, in that such preparation provides a detailed insight into activities linked to critical success factors within the initiating enterprise (Lockwood, 1994, p. 3). 
The same author notes that the choice of a benchmarking partner is not a critical factor. Benchmarking with almost any company will deliver benefits - even if the exercise only serves to confirm what is already being done well (Lockwood, 1994, p. 3).

This view is a little different from mainstream thinking on benchmarking. Many of the contributions in the literature talk in terms of forming benchmarking alliances with "best practice" organisations. Wiarda and Luria $(1997$, p. 3) warn that a lot of disillusionment with benchmarking arises from misconceptions about what it means to be a good performer. They assert that: there is no such thing as the best practice organisation. No company or plant is good at everything." They say that this is true in virtually every sector of industry and that while a particular plant may be near the top of its group for some measures, it will be near the bottom for others.

The new entrant into the benchmarking process may receive a surprise with the outcome. Wiarda and Luria (1997, p.2) state that: "benchmarking is invaluable for its shock value. Time and again, we have seen managers react with disbelief at feedback that tells them their plants are mediocre ... or worse ... at activities that they view as strengths." They suggest that disappointing news should be expected, but that this should be used as an agent for change in the organisation.

Robert Camp (cited in Jay, 1994, p. 34) asserts that many organisations are not familiar with the detailed functioning of their basic business processes. Further, it can be quite difficult to find somebody within the organisation who understands how everything is put together.

The National Industry Extension Service states that the benchmarking process should be adapted to the circumstances of each recipient organisation. The approach taken should be influenced by factors such as:

- The size of the organisation (although more commonly found in large organisations, benchmarking is adaptable to smaller operations). 
- The degree of understanding by the workforce of the strategic plan.

- Whether this is the first time the enterprise has tried benchmarking.

- The sophistication of change management skills in the enterprise.

- The degree of experience with continuous improvement tools.

- The degree of experience and comfort with data gathering and performance measurement, by management and the workforce.

(National Industry Extension Service, 1993, p. 8)

Blewett and Shaw (1995b, p. 242) point out that "each organisation is different and what works for one organisation may not be the best solution for another. In any case, best practice may be a combination of features from different enterprises."

According to the Australian Manufacturing Council (1994, p. 39), their best practice study with Australian and New Zealand manufacturing organisations revealed that "benchmarking is the single practice which most clearly separates Leaders from Laggers."

Gallacher (1991, p. 158) sounded a cautionary note in warning that not everyone in the organisation could be expected to relate to an external benchmarking exercise. For them, a local, internal focus for improvement is appropriate.

Waileck, O'Halloran and Leader (1991, p. 5) ask why, given the galvanising power of the benchmarking process, that many companies and even entire industry groupings do not engage in comparing their performance against some external standard. They suggest that there are three reasons, namely:

- The supposed superiority of invention over copying

- The "we are unique" syndrome

- Moral and legal disapproval of something construed by some as "industrial espionage".

Walleck, O'Halloran and Leader (1991, p. 9) note that benchmarking should not be confused with competitive analysis. The latter focuses on product comparisons, while the former looks beyond products to the 
operating and management skills that produce the products. The same authors caution that: "The most common mistakes benchmarking teams make are to try to gather data on too many topics, schedule too many interviews, and run out of time for analysis of the visits they have made" (Walleck, O'Halloran \& Leader, 1991, p. 19).

Benchmarking, if properly implemented, forces an organisation to compare itself to others, to quantify differences in performance, to document why those differences exist, and identify steps to catch up to and surpass the best in class (Pryor, 1989, p. 29). This outward looking approach is seen as healthy. The same author commented that: "In general, as companies have become larger, they have become more internally focused and often suffer from the 'not invented here' syndrome." Furthermore, "this situation has led to a calcification of the operation of many of those same companies, with poor internal communications and limited responsiveness to customer requiremerits" (Pryor, 1989, p. 32).

Pryor (1989, p. 32) points out however that "a premium must be placed on judgement and insight; the data while pointing the way, will never give you the whole answer. There must be a willingness to estimate and an understanding that benchmarking is not a science."

Tucker, Zivan and Camp (1987, p. 10) offer a further point, in that, "People involved in the benchmarking process often find that the work is broadening and furthers their professional growth. They become more useful to the organisation."

Blewett noted that although the literature on OHS benchmarking is scant, benchmarking is happening between companies (Blewett, 1994, p. 1). Shaw observed that there is a link between benchmarking and performance measurement. She stated that "some approach to assessing or measuring performance is required, both to identify possible benchmarking partners and to measure or rate any improvements which are implemented as a result of benchmarking" (Shaw, 1994, p.17). 
Walleck, O'Hailoran and Leader (1991, p. 17) note that: "for some organisations, an understanding of where change may be most resisted reveals where benchmarking is most needed".

Finally, Lockwood $(1994$, p. 3) has observed that benchmarking delivers a range of useful side-benefits. It provides the workforce with an avenue through which they can express their ideas and thereby help their personal development. Also, the exercise may reveal skills not previously recognised - presenters, inventors, engineers or lateral thinkers.

\subsubsection{Technology transfer - implementation issues}

As indicated earlier, Watson (1992, p. 82) expresses the view that improvement processes may result in continuous incremental gains (sonietimes referred to as "kaizen") or lead to strategic breakthroughs that leapfrog the opposition (sometimes referred to as "hoshin"). It is important to remain alert for opportunities in both the continuous improvement and breakthrough improvement areas.

According to Irwin (1994), there is ample evidence that concepts and innovations that emerge in a particular enterprise, industry or national culture, can be successfully translated and transferred to another culture. The quality of the translation will depend on the level of understanding of the part that different enterprise, industry and national cultures, and techno-cultures, will play in the translation. The effort applied to identifying and influencing organisational and national cultures has emerged as a key determinant of success in the adoption of best practice, Quality Management and other change strategies (Irwin, 1994, p.3).

\subsection{Research Design and Thesis Preparation}

Research involving people is usually divided into two main areas quantitative research and qualitative research.

According to Sarantakos (1993, p. 15), quantitative research is based on deductive logic, it begins from a theoretical base, verification takes place after the theory has been constructed, concepts are firmly defined before 
research begins and generalisations are inductively-based. In contrast, the qualitative research approach (used in this study) is based on inductive logic, begins from a reality base, data generation, analysis and theory verification take place concurrently, flexible concepts are used at the beginning and generalisations are exemplar or analytic.

Both types of research have their strengths and weaknesses. Chadwick, Bahr and Albrecht (cited in Sarantakos, 1993, p. 52) have identified certain strengths attached to qualitative inquiry, namely researching people takes place in natural settings and in the respondent's world, there is greater flexibility and it presents a more realistic view of the world. On the other hand, there may be problems of reliability caused by extreme subjectivity, it is very time-consuming and there may be problems with objectivity and detachment.

Sarantakos (1993, p. 53) commented further that qualitative research emphasises discovery and exploration rather than hypothesis testing, features the active involvement of the investigator in process of data collection and analysis, and is based on analytical or conceptual generatisations only.

\section{$2.7 \quad$ Summary}

This literature review has examined the development of modern management systems and approaches as they relate to OHS, legal requirements, organisational effectiveness and behavioural safety, together with current thinking with respect to performance measurement, auditing, best practice identification, benchmarking and technology transfer. All of these elements are linked, and are relevant to, the present study subject - hazardous materials management.

It is apparent from the literature that there is a strong view that the principles for effective health and safety management and good quality management are the same. Organisations that have embraced the quality principles of leadership, workforce involvement, continuous improvement, performance measurement and have developed supportive organisational 
cultures are usually associated with high standards of OHS performance (Health and Safety Executive, 1992, p. 12). Another key point is that to be effective, chemical safety activities need to be integrated into the normal management functions of planning, organising, leading, controlling and evaluating workplace systems (Winder, 1995, p. 223). Or, as Topf (1997, p. 30) put it, "Safety and environmental stewardship must be integral to the organisation - viewed on a par with other critical management functions such as production, human resources, cost management and quality."

This interdependency and linkage point is important. As Watson (1992, p. 119) says: "One factor that distinguishes the best firms from others is that they see the varlous business tools and systems not as independent solutions to a problem but as a coherent package of change mechanisms." Organisations that exhibit superior performance usually adopt a systems approach to the design of their business, using quality management techniques to complement their strategic planning and business process improvement methods (Watson, 1992, p. 119).

Benchmarking is an established element in a quality management approach. It assists in identifying those aspects of another company's strategies that correlate most closely with successful performance. As Pryor (1989, p. 29) put it, "Often, this analysis will identify some key elements of a successful strategy which you have previously overlooked. By extension, it will yield significant recommendations for how you can alter your own strategy to maximise future performance."

The conceptual framework for the present research is built upon these observations. Furthermore, if it is accepted that performance in OHS is derived from three sources (the people factor, the workplace environment factor and the organisation of work factor), then each of these should represent fertile areas for the benchmarking activity described earlier. The pieces that are missing are the tools and processes to facilitate efficient transfer of ideas, techniques and innovations from one organisation to another. What follows next is a description of the design and conduct of research to address these gaps. 


\section{CHAPTER 3 MATERIALS AND METHODS}

\subsection{Research Methodology}

C. William Emory (cited in Watson, 1992, p. 23) defines research as a "systematic inquiry aimed at providing information to solve problems." Qualitative inquiry techniques have been used in the present study. Patton (1990) has described ten themes of qualitative inquiry. Of these, the one that best describes this work is "personal contact and insight". He defines this as an approach where "the researcher has direct contact with and gets close to the people, situation and phenomenon under study; the researcher's personal experiences and insights are an important part of the inquiry and critical to understanding the phenomenon" (Patton, 1990, p. 40). Further guidance in the area of research methodologies was obtained from Isaac and Michael (1981).

\subsection{Target Population}

\subsubsection{Facilities}

Benchmarking involves a systematic investigation of the targeted process (Watson, 1992, p. 23). The target population for this study was a group of eight mining and mineral processing organisations in Western Australia and the business process under scrutiny was OHS, with emphasis on chemical safety. Several key Industry sectors in Western Australia were represented in the study - that is, gold, nickel, bauxite/alumina, iron ore and chemical suppliers. Facilities were chosen that fall into either the medium or large categories. For these purposes, "large" was established where there are more than 200 employees at the facility and "medium" when there are 50-200 employees. Facilities were chosen on the basis of their size, level of use of hazardous materials and degree of commitment to the quality management philosophies and tools. Diversity in these areas was considered useful. 
Thus, the study focused on conducting comparisons with non-competitors within the Western Australian Mining Industry. These are organisations that are accustomed to working together on common issues in OHS, usually under the auspices of the Chamber of Minerals and Energy of Western Australia. The Chamber has a committee structure that covers central and regional issues and these bodies have played a key role in the significant improvements in safety performance that have been achieved by the Industry in recent years.

As part of the process of drawing up a list of potential benchmarking partners, the researcher consulted with various individuals with an Industry-wide perspective in terms of health and safety. These included the State Mining Engineer, the Mines Occupational Physician and the Manager - Occupational Health at the then Department of Minerals and Energy of WA, together with the Executive Officer - OHS at the Chamber of Minerals and Energy of WA and the former Deputy CEO of the Chamber. Discussion centred on which facilities had strong and/or innovative approaches to chemical safety and were likely candidates for inclusion in the study, based on the considerations outlined earlier. In addition, the study proposal was communicated at various Industry forums and to individuals within the researcher's wider professional network. The eight participating sites emerged from this activity. Only one organisation declined an informal approach to participate. It did so on the grounds that recent staff reductions limited its capacity to respond to the questionnaires.

\subsubsection{Individuals}

An important element of the research invoived structured interviews with senior management at each location, namely with people performing roles such as Registered Mine Manager and Site OHS Professional. In accordance with University policy, application was made to the Human Research Ethics Committee at Edith Cowan University to gain endorsement of the data-gathering methodologies and materials prior to the commencement of the field component of the study. The application included sample letters to the site contacis, an abstract to describe the 
purpose of the exercise and consent forms to cover use of data generated at the particular site. Copies of this material are reproduced in Appendix 5. Ethics Committee approval from Edith Cowan University was communicated to the researcher in a letter dated 14 June 2000.

Thus, in advance of fieldwork at a site the Registered Mine Manager and the Site OHS Professional were approached in writing for their informed consent to participate. The Registered Mine Manager has statutory control and responsibility for all forms of activity at the site and is the appropriate person to give approval for this kind of work. On the other hand, the Site OHS Professional is the key source of detailed information on the management systems that impact on OHS and the use of hazardous materials at the location.

\subsection{Study Design}

\subsubsection{Addressing the Research Questions}

As indicated earlier, the research questions are expressed as follows:

Is there a suite of practical benchmarking tools and methodologies capable of:

- Accounting for the organisation of work factor, the workplace environment factor and the people factor - as they relate to the management of hazardous materials?

- Application to both medium and large organisations?

- Application to other elements under the OHS umbrella, that is, to areas other than chemical safety?

- Identifying pockets of excellence?

- Facilitating the transfer of best practice in the management of hazardous materials?

The variables under investigation in this research are the components of site hazardous materials management programs that relate to compliance with statutory requirements, together with those that relate to "beyond compliance ${ }^{n}$ initiatives in the management (organisation of work), technical (the workplace environment) and people areas. The focus is on critical 
success factors that impact on chemical safety at the location. A scoring system was devised to allow comparisons to be made of the attributes and level of development of programs at different facilities, and for the strengths and weaknesses within a particular facility to be readily identified. Such tools will help with identifying prospective benchmarking partners and should facilitate a focus on "pockets of excellence" that may be suitable for adoption or adaptation at another site.

With regard to the development of benchmarking tools and methodologies capable of accounting for the organisation of work factor, the workplace environment factor and the people factor (the first research question), a set of data-gathering documents was developed for use in the field (see Section 3.5). These were piloted in the early stages of the project (see Section 3.6). Additionally, the material was subject to scrutiny and comment at various points by experts within the researcher's professional network. These measures provided a high level of consensual validity (see Section 3.7.2).

In relation to the second research question (the application of tools and techniques in both medium and large facilities), fieldwork was divided evenly between representatives of the two - to enable valid comparisons to be made.

The third research question deals with application to other elements under the OHS umbrella, that is, to elements other than hazardous materials. For this, an exercise was carried out to develop tools and techniques for use in assessing the status of ergonomic initiatives at a facility level.

With regard to identifying pockets of excellence (the fourth research question), the booklet series and the inquiry that goes with them, were developed to elicit this information.

Finally, in relation to facilitating the transfer of best practice in the management of hazardous materials (the fifth research question), this is a matter that was canvassed with site representatives in the concluding stages of the facility visits. 
The study was cross-sectional in nature and was not designed to track changes as they occur over time at the facility. The focus was on the facts and circumstances available during the contact period with the facility.

\subsubsection{Study Sequence}

Initially, there was personal contact with the Registered Mine Manager (usually the Works Manager) and/or the Site OHS Professional. In most cases, one or both of these people was already known to the researcher as a consequence of his long-term involvement in the development and deployment of OHS policy and standards within the Westem Australian Mining industry. This is an approach suggested by Lockwood $(1994$, p. 3). She notes that: "the most effective way to get a successful benchmarking partner is to know someone within the company. Not necessarily someone in OHS and not necessarily someone at a high level - just someone who can get an interchange happening."

Partner selection and initial discussions were followed by written communications, as described earlier. Subsequently, site visit(s) and phone calls took place to further explain the study objectives, processes, tools, requirements and the benefits of participation and then, importantly, to proceed with the data-gathering and validation effort. Appendix 6 contains a sample program for site visits.

In broad terms, the study sequence was:

- Develop the tools and methodologies

- Approach potential participants

- Conduct pilot exercises at one medium-sized site and one large site

- Assess results

- Modify tools and methodologies

- Conduct the full scale exercise

- Analyse data

- Develop findings

- Communicate relevant firdings and recommendations to study participants. 
The tools and methodologies developed for use in this study were based on the research findings and experience expressed under a variety of headings in the previous chapter. Thus, account was taken of the array of material listed within the References section of this thesis, particularly that relating to quality management and organisational effectiveness, the principles and characteristics of best practice OHS, and compliancerelated issues and guidance.

The various elements may be represented as components of the input model in Figure 11 below.

Study materials and approaches have been influenced by the philosophies and experiences described by leading international researchers and practitioners (see Sections 2.1 and 2.3, in particular). This provides a high level of content validity and generates confidence that research tools and processes are themselves in line with best practice.

\subsection{Development of Tools and Methodologies}

Eight booklets were developed for use in the study and copies of these are provided in Appendix 7. Particular attention was applied to the layout and content of the booklets. As Webster (2000, p. 4) points out, "the construction of questions that elicit respondent cooperation and contain complete and accurate information is as important as any component of your research study." She says that extreme care must be applied to make sure that that design and format aspects demonstrate professionalism, quality and attractiveness. On the last point, she asserts that the appearance of the questionnaire will impact greatly on the level of response to the material (Webster, 2000, p. 5). She comments that there are four key matters to be considered in developing the questionnaire:

- Inclusion of items that are pivotal to the study.

- Obtaining maximum cooperation rates.

- Ensuring that the questions match the capacity of respondents to answer reliably. 
- Minimizing item non-response and sample bias (Webster, 2000, p. 4).

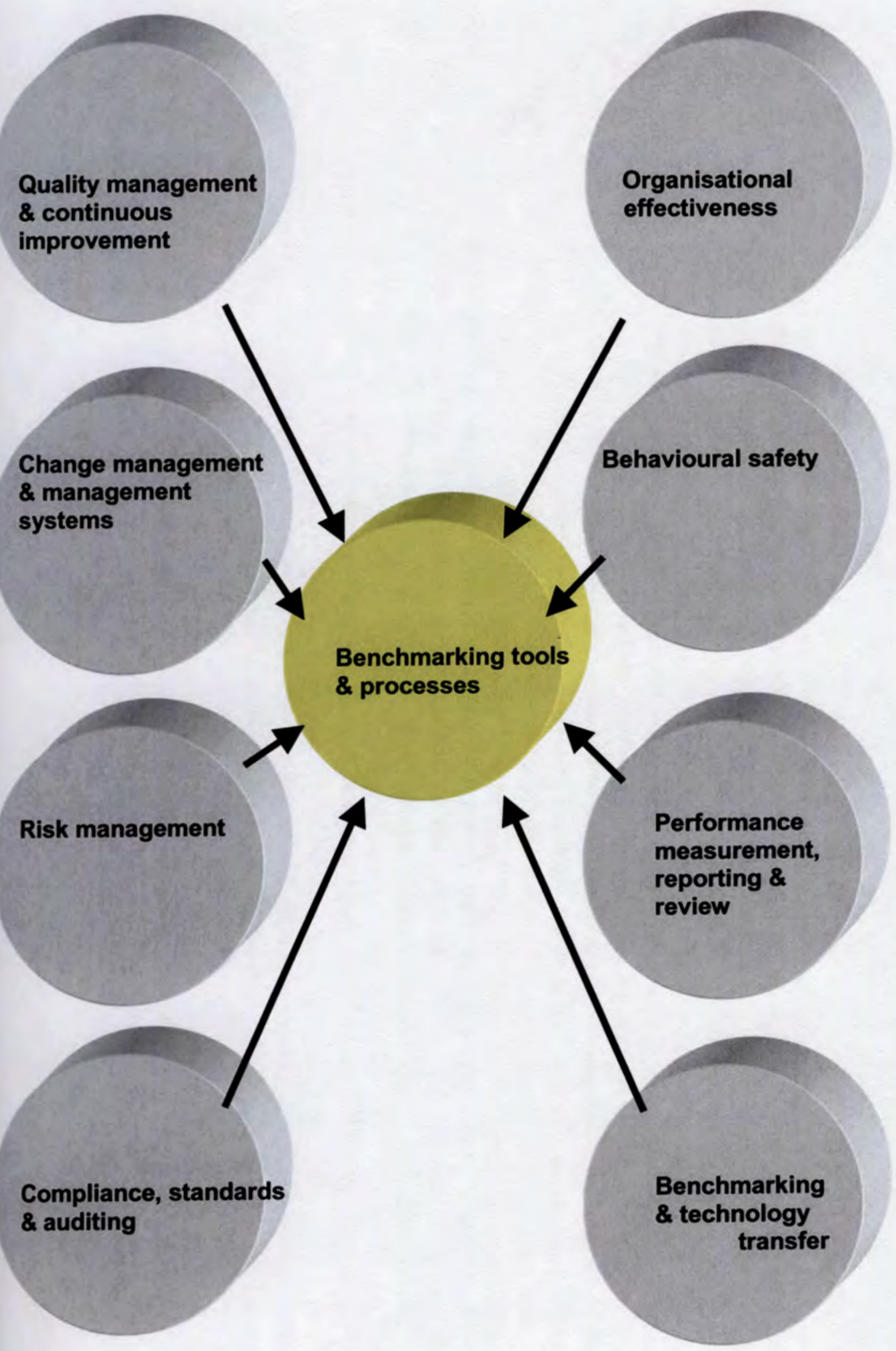

Figure 11: An input model for benchmarking processes and tools 
The first two booklets were part of the initial information-gathering activity. That is, they were directed to the location in advance of the first visit by the researcher and results were used to shape the inquiry and assist with making time on-site as productive as possible. The next four booklets were used to assist discussion and the gathering of data during site visits by the researcher.

Booklet 3 contained compliance-related material. Content was derived from the requirements expressed in relevant sections of Western Australia's Mines Safety and Inspection Regulations (1995). The "beyond compliance" material relating to organisation of work, the workplace environment and people initiatives (Booklets 4-6) was developed from the researcher's professional experience, together with a review of the literature on management systems and continuous improvement. Benchmarking methodologies or processes were derived principally from the literature on quality management and auditing practice.

Data collection during the site visit phase was based on the three qualitative inquiry methods, namely in-depth, open-ended interviews, direct observation and review of written records. The latter two approaches were employed to verify what has been claimed during the initial interviews and to improve face validity:

The final two booklets were used to assemble results and findings - for feedback to each participating organisation.

A Pareto approach was adopted in relation to the issues pursued in the field. In other words, it was seen as important to avoid getting immersed in complex and very detailed pursuit of minor aspects of chemical safety. Far better to focus on the $20 \%$ of issues that cause $80 \%$ of the problems in this area. Thus, a balance was sought in getting sufficient information to draw valid conclusions about chemical safety at the site, without the exhaustive pursuit of minutae. The latter would have created a log-jam of information and a major barrier to future deployment of the tools and methodologies being exercised in this work. 
The questions that are raised in the booklets were directed at senior site personnel. They are based on the four fundamental assumptions expressed by Waterhouse (1992, p. 16), namely:

- Management knows that certain minimum standards have to be achieved.

- They have in place a system for achieving them.

- A means of monitoring the achievements is in place.

- It is used.

This awareness issue is one of three assumptions that underpin the design of this study. These are:

- That the senior manager and the OHS professional at the location will be aware of innovations and exceptional practice, as may exist in certain areas of the mine or plant.

- That proceeding through the structured sequence of booklets developed for this study will flush out "pockets of excellence" that have potential to be of use in a two-way exchange between benchmarking partners (whether this be within the same organisation or between different organisations).

- That almost all sites have something to offer (as suggested by Lockwood, 1994, p. 3).

The data collection instruments mentioned above are described in terms of their purpose, structure, application and interpretation of data as follows:

\section{- Booklet 1: Site Profile}

This document is designed to record contact information, to elicit basic information on what business is conducted at the facility, to gain an appreciation of how workforce members (employees arid contractors) are deployed, to identify the key OHS issues at the site and to describe how these are addressed.

The booklet was part of the initial package of materials sent to the participating location - with a request that it be completed and returned to 
the researcher before the initial site visit. Its contents made a good starting point for subsequent discussions with the Registered Mine Manager and/or the Site OHS Professional. Data gathered with this document has been presented in a modified way in this thesis - to protect the identity of participating organisations where this is desirable and practicable (see discussion under "Limitations").

\section{- Booklet 2: Preliminary Questions}

This booklet was also part of the initial package of materials sent to the participating location. It seeks to gather preliminary information on the way OHS, in general, and chemical safety, in particular, are managed on the site. Again, data gathered in this way were used to focus the inquiry with a view to making site visits as productive as possible.

Part $A$ is directed at OHS in general. It consists of a mix of yes/no questions, some open-ended questions, a tick-the-box self-ranking of performance question and an invitation to nominate potential benchmarking partners.

Part $B$ is directed more specifically at chemical safety. it features a set of yes/no questions, a request for a flow chart description of how chemicals are managed on site (thereby creating a possible line of inquiry). Furthermore, it contains an invitation to nominate critical success factors for chemical safety at the site, together with performance measures and innovations that have been put in place.

\section{- Booklet 3: Compliance}

This booklet is designed to assist in establishing the level of compliance at the facility with Part 7, Division 3, of the requirements of the Western Australian Mines Safety and Inspection Regulations 1995. This is the element that deals with the management of hazardous materials. The booklet does not attempt to cover matters of a more general nature, such as statutory duty of care responsibilities, appointment and training of safety representatives, OHS committee structures and similar - 
notwithstanding that these will have an impact on OHS outcomes at the facility. The document was used to initiate discussion with the Registered Mine Manager and/or the Site OHS Professional.

\section{- Booklet 4: Organisation of Work}

This document was designed to flush out local initiatives that might qualify as "pockets of excellence" with respect to management enablers for OHS. The focus here is on the enablers of leadership, commitment, planning, organisation, training, communication and measurement. All of these elements feature in the material described in the Literature Review completed earlier and they are strongly linked by numerous authors to effective management of OHS and of the enterprise as a whole (Toohey, 1987, p. 238; Strobach, 1990, p. 42; Fisher, 1991, p. 27; Deacon, 1994, p. 19; Worksafe Australia, 1995, p. 13).

The practice was to direct a self-assessment questionnaire to the Works Manager or site OHS professional to establish a rating ("Poor", "Fair", "Good" or "Exceilent") in various key areas. A "Poor" rating means the element is absent or hasn't been considered in the past. A "Fair" rating is applied when programs or efforts are still at an early stage of development or are carried out sporadically. A "Good" rating is used for solid, welldeployed efforts and "Excellent" is ascribed to outstanding programs.

When the "Good" or "Excellent" rating was nominated, then there was a follow-up question that invited a description of what initiatives gave rise to this opinion. Items so identified were then singled out for more detailed discussion, followed by the verification steps mentioned earlier. Where "Poor" or "Fair" were nominated, no further enquiry or action took place.

\section{- Booklet 5: The Workplace Environment}

This document was used in a similar way to the previous one. Here, though, attention was directed at the technical elements within a risk management framework, namely hazard identification, risk assessment and risk control - terms equivalent to those used for many decades to 
describe the elements of Industrial Hygiene activity, namely recognition, evaluation and control. In more recent times, the sequence has become familiar to the public in Western Australia as "Spot the Hazard, Assess the Risk, Make the Changes" - as promoted by Worksafe Western Australia (1995, p. 14).

As indicated earlier, the document was designed to help identify efforts and innovations that go beyond the letter of the law and provide potential benchmarking opportunities with respect to activities within the workplace environment. The sub-elements used in this booklet provided a checklist during initial contact with the Works Manager and/or the Site OHS Professional. Inquiry centred on whether the element was present and, if so, in what form.

The document includes an element on the characteristics of industrial Hygiene programs in three stages of development, namely "beginning", "improving" and "advanced", and has been derived from the researcher's long-term involvement in this field. There are 11 descriptors of a Stage I (beginning) program, 13 descriptors of a Stage II (improving) program and 20 descriptors of a Stage III (advanced) program.

An overall score was generated: $50 \%$ for no progress bayond Stage 1 , then a sliding scale up to $75 \%$ for implementation of Stage II descriptors and a sliding scale up to $100 \%$ for implementation of Stage III descriptors. Thus, if inquiry and verification reveals that the site is clearly in Stage III and that all but two of the advanced elements are in place, then the facility score would be $100 \%$ minus $2 \times 1.25 \%$, or $97.5 \%$. There was no attempt to apply weighting to individual items listed on the form.

- Booklet 6: People Initiatives

This booklet was designed to seek out exceptional practice or innovations with respect to people factors. The nominated activities figure prominently in the literature described earlier and are likely to be associated with superior performance in OHS - a premise that was tested during the study. As with the other lines of inquiry mentioned earlier, there might be 
local initiatives that fall outside of the points on the list but represent potential benchmarking opportunities for other organisations. Such items were identified and captured during the interview stage or subsequently during observations that took place in the field.

- Booklet 7: Site strengths and opportunities for improvement

This booklet was designed to provide the location with an analysis of data gathered during the preliminary contact phase and subsequent site visit (s). It presents an appraisal of programs and processes relating to OHS in general, and chemical safety, in particular. It contains broad statements about each of these elements, together with an outline of strengths and opportunities for improvement under the headings referred to eartier namely, compliance, organisation of work, the workplace environment and people initiatives. The "opportunities for improvement" that are presented in this booklet are based on what the safety literature is identifying as being important to OHS efforts. In other words, there is no attempt in Booklet 7 to draw comparisons with what other participating facilities are doing in the OHS area - this comes in the next booklet.

Booklet 7 contains a bar chart entitled "facility profile". The intention of the chart is to provide a snapshot of the relative strengths of the four elements mentioned above. The compliance score is derived from the percentage of Yes/No responses recorded in Booklet 3, where these have been supported by verification in the field, or via document review. The scores for the other three elements are based on interviewee response to questions raised in Booklets 4,5 and 6 . Under each sub-fieading, a rating of "Good" or "Excellent" registered as a score. Thus, if there were 6 of 12 rating questions that attracted a "Good" or "Excellent" then the score would be $50 \%$.

- Booklet 8: Scoresheets, inter-site comparisons and benchmarking opportunities 
This booklet provides scores for each of the four elemerits that have been reviewed, namely degree of compliance with statutory requirements, together with the "beyond compliance" areas covered by management enablers (organisation of work), technical initiatives (the workplace environment) and people initiatives. An overall facility score was derived from this. In addition, the booklet provides graphical comparisons of performance at the different sites - to enable locations to see where they sit in relation to peer operations elsewhere in the Western Australian Mining Industry. Finally, a table is presented that identifies where benchmarking opportunities appear to exist within the participating facilities.

\subsection{Data Collection, Analysis and Communication Procedures}

As indicated earlier, initial contact with organisations identified as potential study participants was via a personal approach to the Registered Mine Manager and the Site OHS Professional. In several cases, these people were already part of the researcher's professional network. During this initial interaction, the objectives and deliverables of the study were outlined, along with a description of how the research might be conducted at the site. The Powerpoint presentation materials provided in Appendix 4 were used to assist the briefing process.

Once there was agreement in principal to participate, a more formal approach was made with written communications similar to those provided in Appendices 5 and 6 . Both the Registered Mine Manager and the Site OHS Professional were made aware of the benefits of participation (for the site) and were given assurances in terms of privacy and security of data. Additionally, the Registered Mine Manager was asked to indicate on a reply slip whether data generated at the site could be used for the present study only or for this and any subsequent research.

Once written agreement to participate was obtained from the site there was further contact to confirm plans for the first visit. This covered travel and accommodation, if relevant, site requirements (such as safety rules 
and procedures) and a program for the visit. In addition, a package of materials was despatched with a request that this be completed in advance of the visit - to make the time on site more productive.

The initial visit typically began with a meeting with the Registered Mine Manager, the Site OHS Professional and other members of the Management Group - to review the program and to discuss broader aspects of OHS management systems, activities and performance. This was followed by a longer session with the Site OHS Professional to discuss his/her response to the preliminary package of materials that had been completed in advance of the visit, and to work through the detailed questions and forms that are described elsewhere in this document. Field visits were then conducted to verify the information that had been obtained earlier. These featured a "walk-through survey". This is a traditional Industrial Hygiene technique used to effect a preliminary assessment of potential hazards in the workplace. As Harvey (1980, p. 3.2-01) notes, the walk-through survey is used to establish:

- The hazards of the workplace that may give rise to ill health or discomfort.

- The likely magnitude of such identified hazards.

- The control measures which are deployed for each hazard.

- The procedures that are in place to maintain the control measures.

- The monitoring that is applied to workplace hazards.

The walk-tirrough survey relies on the experience of the $\mathrm{HH}$ professional and on use of the senses. During this part of the exercise, the researcher used visual means to identify sources of contamination, to assess the state of housekeeping and to note any contaminants on the face, hands or clothing of workers. In some instances, the sense of smell was applied to the detection of gases and vapours in working areas.

The walk-through survey also provided the opportunity to seek further detail from the OHS Professional and to examine the suitability and deployment of Material Safety Data Sheets, labels, waming signs and personal protective equipment in the workplace. 
Thus, methods used for this phase were those commonly applied to audising, namely inquiry (formal and informal questioning), observation (physical examination) and verification testing (scrutiny of records and similar). This was followed by a further session with the Site OHS Professional - to address any gaps in information and to verify some points. Preliminary data was then collated and analysed.

Subsequently, there was an option to return to the site to address any inconsistencies or gaps, to verify findings and to provide further feedback to counterparts. This was followed by finalisation of data and the drawing up an account of the findings. Each participating facility received a candid report on data generated at its site - both in terms of strengths and opportunities for improvement. The final step was to share results derived from the broader group, in a manner similar to that shown in Booklet 8 .

This process was piloted during the early stages of the project. Piloting focused on one medium-sized site and one larger site. Adjustments that followed were of a minor nature and principally related to the way questions were directed to the interviewee.

\subsection{Reliability and Validity of Study Processes and Tools}

\subsubsection{Reliability}

According to Jansz and Nedved (2002, p. 22), "Reliability is the degree of consistency, or dependability, with which the instrument measures the attributes it is designed to measure." In terms of the present study, this refers to the ability to obtain the same results on repeated application of the benchmarking tools or processes at a given facility. Jansz and Nedved (2002) have outlined several ways to test reliability. For the present purpose, research data was tested for reliability by having the people who contributed the information at each facility establish that transcripts, records and the researcher's interpretation of what had been provided were accurate. Raw information and early observations and ideas were summarised and shared at the conclusion of the site visit, before departure. Subsequently, visit outcomes were collated and 
checked with the host site before a final report, similar to the sample version provided in Appendix 8, was made available to the location. Additionally, the booklet structure was designed to ensure that questions were clear and that data were collected in a methodical and consistent way.

\subsubsection{Validity}

According to Jansz and Nedved (2002, p. 24): "Validity is the degree to which an instrument measures what it is intended to measure" and, furthermore, that "there are several types of validity." The Jansz and Nedved (2002) reference provides a description of face validity, content validity, consensual validity, criterion validity, construct validity, internal validity and external validity.

Face validity is the extent to which the research tool appears to give logical answers or is based on objective, verifiable evidence (Jansz \& Nedved, 2002, p. 24). The process used in arriving at potential benchmark opportunities began with a review of site literature and responses to the initial set of questions (Booklets 1 and 2). This was followed by the interviews that took place with senior site personnel during the facility visit. Importantly, the next step was one of verification - using observation, inquiry and review of documentation as the principal tools. This imparts a high level of face validity to the research.

Content validity is related to the thoroughness, or completeness, of the research measuring tools and the extent to which underlying concepts of the research question have been canvassed (Jansz \& Nedved, 2002, p. 25). The literature review described in Chapter Two has sought to capture contemporary views and experience on what constitutes best practice in OHS, what factors impact on this, and how to identify and measure the extent to which this prevails in an organisation (see Section 2.5, in particular). A very clear conceptual framework emerged after the literature was reviewed. The role of management enablers (organisation of work), technical factors (the workplace environment) and human factors in accident causation and in preventive efforts was recognised and became 
a theme for all that followed. The various elements that provided input material for the development of tools and processes are represented as a model in Figure 11, presented eartier.

Consensual validity was achieved by sharing newly developed materials and methodologies with experts in the respective areas. For the broader study, ideas and products were discussed at various points with senior OHS professionals in Industry and Government. For the smaller study dealing with the ergonomics booklet (Appendix 9), a panel of experts was assembled to review the product for clarity, appearance, ease of use and potential usefulness as a tool for technology transfer. Results are discussed later.

Concurrent validity is the ability of the research tool, or research design, to measure current observable behaviour - the latter is measured against objective data available at the same time (Jansz \& Nedved, 2002, p. 25). The study has sought to develop a scoring system to reflect the innovation and level of energy that is being applied at the site and to relate this to a widely used measure of OHS performance, namely the outcome measure of Lost Time Injury Frequency Rate (LTIFR), as used throughout Industry in Australia.

Finally, the study design carries an element of external validity. The latter refers to the researcher's ability to generalise the findings to the larger population from which the sample was drawn (Jansz \& Nedved, 2002, p. 27). The participating locations were recruited from a diverse group of mining, mineral processing and related industry operations in Western Australia. There was diversity in such things as geographical location, size of the operation, type of ore being mined or processed and level of sophistication in terms of management systems, OHS programs and procedures. It was felt that successful application of the study tools and processes against that backdrop would provide confidence that a wider scale application would be viable. 


\subsection{Deliverables for Participating Organisations}

Each facility that took part in the study received two packages of information. The first was a collation of material gathered at the location via the use of Booklets $1-6$, together with the results of walk-through surveys, inspection of documents and other verification activities. This provided a comparison between what the facility appeared to be doing in terms of OHS, in general, and chemical safety, in particular, and what the literature appears to be representing as best practice. It provided the facility with an assessment of "strengths" and "opportunities for improvement" under the key headings of "compliance" (with legislative requirements), "organisation of work", "the workplace environment" and "people". It concluded with a summary of findings and preliminary recommendations. A sample report (to an imaginary facility) is provided as Appendix 8. it was the practice during the field exercises to try to direct the first feedback package to the participating site within one week of the main site visit. The report was directed to the participating site alone.

The second package was focused on inter-site comparisons. As such, it wasn't ready for circulation to the participating locations until after the final site visit had been conducted. For this package, sites were referred to as Facility One, Facility Two, and so on - to preserve anonymity for those sites that preferred this to be the case. Facilities were compared in terms of generic information, including scores obtained for the four headings mentioned above. Importantly, their respective benchmarking opportunities or "pockets of excellence" were identified and summarised for both OHS in general and for chemical safety. Where a site subsequently expressed interest in following-up on a knowledge transfer opportunity with another facility outside of its wider organisation, then contact details were provided after permission had first been obtained from the prospective benchmarking partner. 
One of the research questions is directed at establishing whether the tools and processes may be applied successfully to OHS elements other than chemical safety. A small supplementary exercise was conducted to investigate this question. A booklet was developed to stimulate discussion and gather information on the organisational, technical and people measures that the participating site applies to the management of ergonomic risk. Its purpose was to identify innovative, cost-effective and practical approaches to addressing such risks.

Ergonomics was chosen as the topic for this exercise since strains, sprains and other forms of musculoskeletal injury and disease dominate the injury patterns experienced by Industry (Department of Consumer and Employment Protection, 2002; Minerals Council of Australia, 2002; Department of Minerals and Petroleum Resources, 2002; Alcoa, 1999a; Department of Industry and Resources, 2003).

The ergonomics booklet, "Ergonomics - In Pursuit of Best Practice", was fashioned after the booklet series used in the main study and a copy is provided as Appendix 9. Technical content was derived from various internal and external guidelines and standards (Alcoa, 2001a; Worksafe Western Australia, 2000; Worksafe Australia, 1993). Like the other booklets, it begins with a series of yes/no questions to establish whether important program elements are in place, before moving to some openended questions. This is followed by a section dealing with self-rating of certain program elements that are strongly advocated in the mainstream safe'ty literature. Again, the approach is designed to flush out "pockets of excellencc" and to identify potential benchmarking opportunities.

The booklet was tested with a panel consisting of an occupational physician, together with four Physiotherapist/Ergonomists responsible for leading much of the ergonomic activity at their respective refinery and mine site locations in Western Australia. They were asked to rate the booklet in terms of format and appearance, clarity, ease of use and potential value as a tool for future benchmarking activity. The same four- 
level scale that is used throughout this work ("Poor", "Fair", "Good" and "Excellent") was applied to these deliberations. Utilisation of an expert panel in this way provided the booklet with consensual validity (Jansz \& Nedved, 2002).

\subsection{Limitations}

At the beginning of the study, it was recognised that it would be very unlikely to encounter many Western Australian organisations with highly developed, wordd-class systems for managing hazardous materials. However, it was expected that "pockets of excellence" would be found within most, or all, of the facilities taking part in the study. The challenge would then be to suitably identify and focus on these opportunities.

Another issue likely to be encountered was that of confidentiality. The study indicates the position of the facility on the continuum towards excellence in occupational health and safety, and in the management of hazardous materials, in particular. This raises the prospect of significant organisational weaknesses being highlighted and, further, that the study will reveal areas where there appears to be non-compliance with statutory requirements. It was recognised that this may be embarrassing for some of the organisations involved and assurances of confidentiality might be needed. Every effort was made to disguise the identity of participating sites, notwithstanding the difficulty in achieving this in the context of the nature and size of the Industry in Western Australia. Having said that, none of the participating sites raised any concerns about the confidentiality aspect and all of them seemed to be open to a "warts and all" approach to the review.

As mentioned earlier, the study was cross-sectional in nature and was not designed to track changes as they occur over time at each facility. The study represented a "snapshot" of facts and circumstances, as they existed at the time. Also, it relied heavily on the knowledge of a few key site individuals and their ability to recall and describe relevant processes, tools and initiatives at the site. On the latter point, the verification activities 
that were part of each site visit were designed to improve the reliability and validity of the results and to limit any problems associated with subjectivity of responses and recall.

Despite these limitations, the study tools and processes were fully deployed with participating sites. Results are given in the next chapter. 


\section{CHAPTER 4 RESULTS}

\section{$4.1 \quad$ Overview}

Results from fieldwork are assembled in facility order. The sub-headings used below are derived from the principal headings used in the booklet series. Much of the material that follows has been taken from entries in the booklets and from supplementary notes that were taken during interviews, inspection of documents and other field verification activities.

\subsection{Facility One}

\subsubsection{The Operation}

Facility One was a Bayer Process alumina refinery processing bauxite from the Darling Ranges. The main raw materials for the facility are bauxite, caustic soda, natural gas, starch, synthetic flocculants, lime and sulphuric acid. The main end product is calcined, smelting grade alumina, a material that is shipped overseas and interstate for use in the Hall Heroult process to produce aluminium metal. Some "chemical" grades of alumina are also produced for specialised applications.

The principal waste material is bauxite residue. Since Darling Range bauxite is of a low grade by world standards, a large proportion (twothirds) remains after the available alumina component has been extracted. This is then subject to various stages of washing to remove as much as possible of associated caustic soda before being directed to the residue storage area for drying and long-term storage in purpose-built, PVC-lined containment areas.

A simplified description of the Bayer Process, employed at Facility One, is provided in Figure 12. 


\section{Bauxite from Minesite}

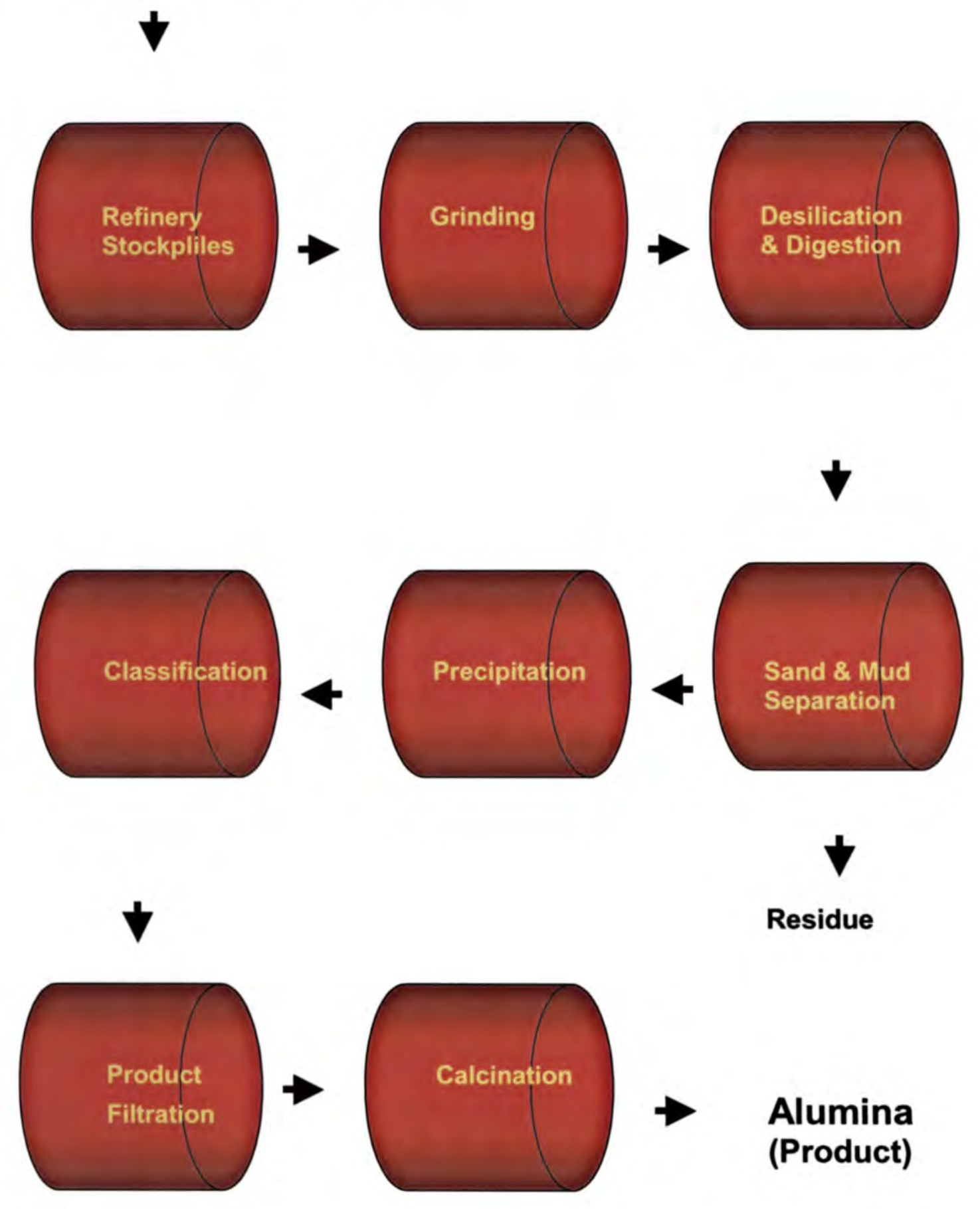

Figure 12: Schematic flowsheet - bauxite to alumina (Facility One)

\subsubsection{The Workforce}

The workforce numbers 960 full-time, permanent employees and 110 fulltime temporary staff. Company employees are split between 350 in the administration and management group, and 720 wages employees in 
production and maintenance jobs. Approximately half of the workforce is on a rotating shift pattern (12 hour shifts or 10.3 hour shifts). There are 30 contracting organisations on-site - mostly engaged in maintenance activities.

The location has a very low rate of labour tumover and there are many employees with more than 10 years of servit:e at the site.

\subsubsection{Organisation for OHS}

The location has a full-time occupational physician (overviewing this site arid others), a visiting occupational physician (two days per week), together with three full-time occupational health nurses and a physiotherapist/ergonomist. Visiting podiatrists, nutritionists and other specialists provide further support for health efforts. An Industrial Hygienist is responsible for recognition, evaluation and control of the various physical, chemical and biological hazards in the workplace, and she plays a major role in the chemical safety activities on site. There is an EHS manager who coordinates environment, health and safety activities on the site. Four full-time safety resources are allocated to the various business centres. These people are supported in their efforts by a strong safety representative system (about 40 on site) - drawn from the ranks of each natural work group. The underpinning philosophy at the site, however, is that safety is a "line" responsibility and that safety professionals are there to act as a resource only.

\subsubsection{Principal OHS Issues and Challenges}

- Physical hazards: Noise, vibration, ionising and non-ionising radiation, and thermal stress.

- Chemical hazards: Alumina dust, bauxite dust, asbestos fibres, alkaline mists, heavy metals, welding fume, combustion gases and industrial chemicals (abrasives, compressed gases, cleaning agents, oils and greases, surfactants, adhesives, sealants, solvents, surface coatings and similar).

- Biological hazards: Bio-aerosols associated with cooling tower operation. 
- Ergonomic hazards: Lifting, pushing, pulling: twisting, awkward postures, repetitive motion and similar have potential to produce ergonomic injury.

\subsubsection{Other Relevant Information}

The facility belongs to a global metal business that has a high lev. nf vertical integration, that is, the broader organisation is active in all $m_{h}$. segments of the industry: mining, refining, smelting, fabricating and recycling. As such, it obtains leverage and benefits from the OHS knowledge, systems, procedures, tools and resources that reside within the wider organisation.

The Department of Minerals and Petroleum Resources (DMPR) conducted an audit of management systems at Facility One in April 2002. The site obtained a score of $89.7 \%$ - based on the structure described by DMPR (2002) and alluded to earlier. Figures provided by the State Mining Engineer (M. Knee, personal communication, March 14. 2003) indicate that 128 mine sites, mineral processing and transporthandling operations in Western Australia have been audited in this way over the past five years. Scores ranged from $35 \%$ to $99 \%$, with a mean of $76 \%$.

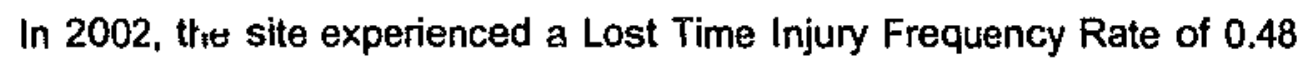
per $1,000,000$ hours worked.

\subsubsection{Organisational Effectiveness}

The questions posed in Booklet 2, under the heading "general OHS", are designed to provide a preliminary gauge of organisational effectiveness. The location representative responded in the affirmative to all nine questions. Thus, the facility has in place:

- A clearly expressed OHS policy.

- A philosophy and commitment to exceed minimum standards.

- Integration of OHS into the key business functions of planning, organising, leading, controlling and evaluating. 
- Clearly defined OHS performance expectations and measures for all levels in the organisation.

- Injury management plans.

- Clear evidence of management involvement and commitment to OHS.

- Clearly assigned OHS responsibilities for managers and supervisors, expressed in terms of their normal duties.

- Commitment and involvement of employees, with formal and informal structures to encourage their participation.

- A generally held view within management that Corporate investment in OHS is good business practice.

\subsubsection{Benchmarking. Best Practice and Technology Transfer}

The location representative indicated that previous efforts with respect to external OHS benchmarking have been of a very limited nature - of a type characterised by site visits and unstructured discussions on a few items of interest. Some attempts had been made within the wider organisation to identify best practice for certain elements and some site visits resulted from this. Technology transfer is actively encouraged within the wider organisation and auditing processes assist with this. An Intranet website on ergonomics was cited as an example of an initiative to encourage facilities around the world to share their ideas and successful outcomes.

\subsubsection{Self-Ranking in OHiS}

The location representative thought that her site would rate in the top five percent of sites within the Mining Industry of Westem Australia. She based this on her knowledge of systems and procedures in place at her site, together with the low incidence of chemical-related injury and nearmiss experience at the facility. She also had some anecdotal information from her external professional network and from suppliers and others who move from site to site.

\subsubsection{Potential Benchmarking Partners}

The DuPont organisation was put forward as the company most admired in terms of performance and reputation for excellence in OHS. Within 
Westem Australia, the Woodside gas operation at Karratha was seen as a superior performer.

\subsubsection{Chemical Safety Preliminary Questionnaire}

In relation to the chemical safety questionnaire presented in Part B of Booklet 2, the location representative responded to ali elements in the affirmative. Thus, the facility is characterised by the following:

- A published policy on the management of hazardous materials.

- Roles and responsibilities have been clearly assigned.

- A current hazardous materials inventory is available.

- Material Safety Data Sheets (MSDSs) are available for every chemical entering or leaving the site.

- An MSDS will normally accompany the first supply of a hazardous material to the location.

- There is interaction between the Purchasing and Safety/Health groups to screen new materials requested for purchase.

- There is a system to prevent non-approved chemicals coming on-site and for ensuring that those chemicals that are on-site have been approved.

- There is a system to track where and how much chemical usage occurs on-site.

- The site complies with all relevant legal requirements for the safe storage, transport, issue, use and disposal of hazardous materials employed or generated by the on-site operations.

- Labels and warning signs are posted for every hazardous material used on-site or sent elsewhere from the site.

- Contractors are expected to meet similar requirements and standards with respect to the hazardous materials they bring onto the site.

\subsubsection{Flow Chart}

There are many controls built into the process for procurement of hazardous substances. These cover access through the Stores requisition system, by purchase requisition and by direct ordering through 
a Supply (CLIPS) catalogue of approved hazardous materials. Purchases by petty cash or credit card, or use of free samples are not available without authorisation by the Supply Department or the Site Industrial Hygienist. The latter individual carries out a risk assessment and, where appropriate, arranges for a Job Safety Analysis to be performed before the material comes onto the site. She actively discourages the advent of new materials where existing products are adequate for the task at hand.

Figure 13 represents a simplified version of the steps used at Facility One to vet and control access of new chemicals to the site.

\subsubsection{Critical Success Factors}

The site representative was asked to nominate the four most critical success factors in relation to chemical safety at the location. She nominated:

- An appropriate organisational structure and resources to service the hazardous materials management area.

- Leadership, visibility and support from Management.

- Clearly enunciated procedures, together with good training and communication.

- A well maintained register and auditing function.

\subsubsection{Performance Measurement}

The traditional lagging indicators of OHS performance are quite prominent at the facility. There is a close focus on Lost Time Injury Frequency Rate (LTIFR) - a measure used widely by Government and Industry organisations throughout the country. Also, an internal measure, referred to as "Total Recordable injury Frequency Rate (TRIFR), is used to track all injuries more serious than a first-aid injury. This covers injuries that require some form of medical attention beyond first-aid treatment, injuries that restrict the individual from performing all elements of the job and lost time injuries. The ratio of injury-free events to the all-injury count is used to encourage open reporting of near-hit events or situations that pose a hazard and require some form of corrective action. 


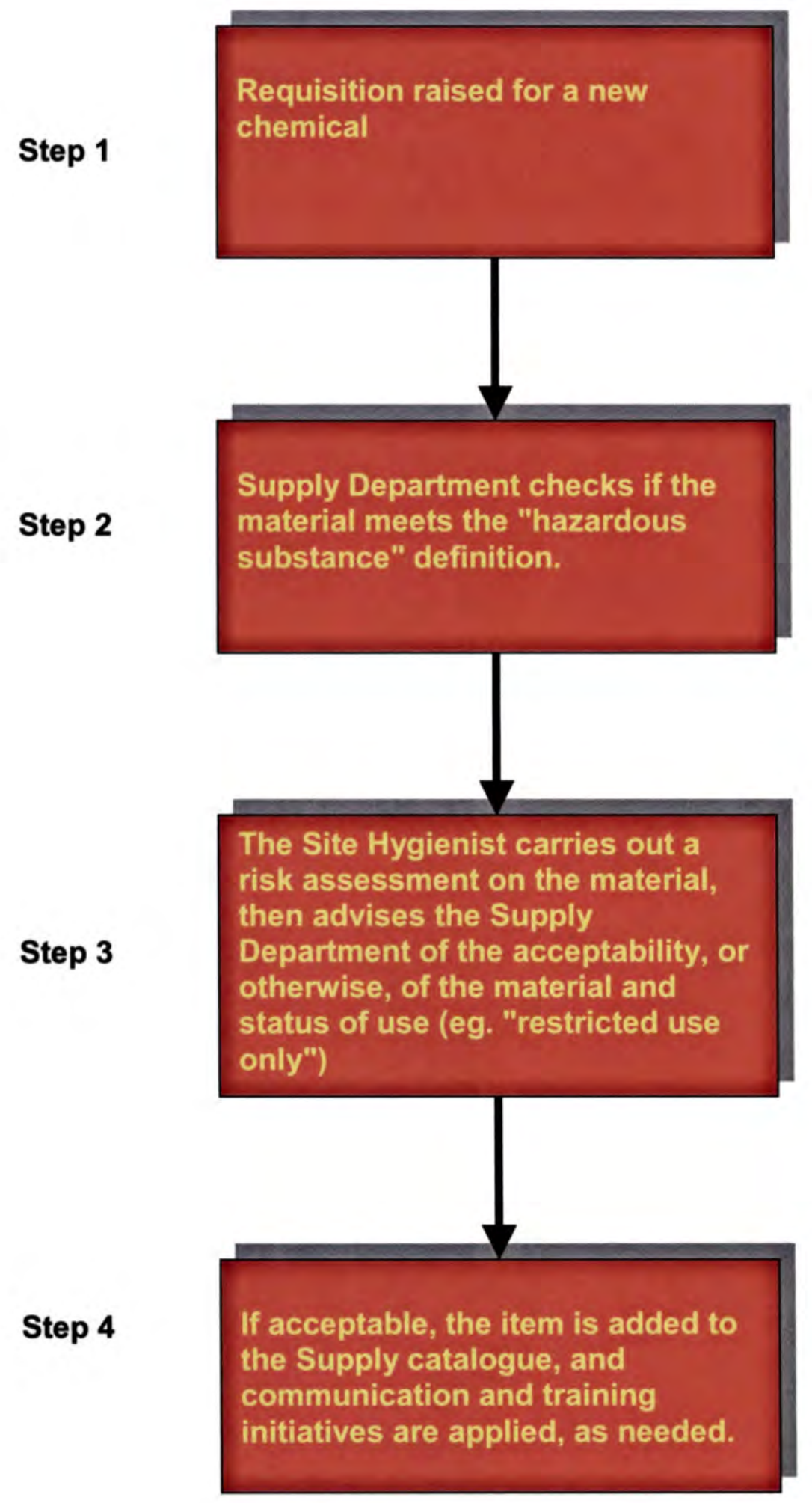

Figure 13: Acquisition of new chemicals - Facility One

Leading indicators of performance have been applied in several areas of OHS. DuPont-style management safety contacts are deployed throughout the facility. These are highly visible examples of leadership and 
commitment, and are formalised, measured and reported on a regular basis. They constitute an element of the performance appraisal structure for line management personnel. Task observation programs, supported and deployed by shop-floor employees are also subject to formalised measurement. Such measures reflect compliance with procedures arid rules, and are part of bigger behavioural safety efforts at the site.

Chemical exposures are managed and measured via a system that has people in similar exposure groups (SEGs), as described by Mulhausen and Damiano (1998). Corporate targets are set for the reduction in number or magnitude of unacceptable chemical SEGs and progress is tracked on a monthly basis. The term "unacceptable" is applied when $5 \%$ or more of the sample results are above the occupational exposure limit for the agent involved. Thus, the approach is very conservative and protective of health.

A Safety Performance Matrix, similar in format to that developed originally by the Eastman Kodak Company, and described earlier, is deployed as a means of tracking progress with a collection of several leading and lagging indicators.

\subsubsection{Innovations in Chemical Safety}

The location was able to demonstrate many innovations that could be of potential interest to other organisations.

Details are provided in the sections that follow but, in brief, these cover:

- Strategic and tactical planning templates.

- Well-deployed procedures for vetting new chemicals and tracking their use and consumption rate on-site.

- Job dictionaries and similar exposure groups (SEGs).

- Qualitative exposure assessments, supported by Hygenius software.

- Quantitative exposure assessments, supported by Hygenius software.

- Measurement and reporting of performance in chemical safety.

- Risk mitigation initiatives.

- Employee involvement and communication efforts. 
- Medical surveillance.

\subsubsection{Compliance}

This element was examined with the aid of Booklet 3 (Appendix 7) and the Western Australian Mines Safety and Inspection Regulations 1995.

- Reg. 7.21: The Material Safety Data Sheets (MSDSs) held on site are in the Worksafe Australia format and are current (by definition, less than 5 years old). An external party that specialises in this area (Chem Alert) provides most of these. Some internally generated sheets for site products and by-products supplement them. Additionally, there is a compilation of original, hard copy MSDSs from suppliers - covering past and present use of materials on the site.

- Reg. 7.22: Containers are generally of suitable design and manufacture. There was some variability around the site in terms of the practices surrounding the transfer of bulk supplies to more suitable smaller containers.

- Reg. 7.23: Many of the more substantial containers that are used on site are returned to the supplier and are then recycled. Those that remain are disposed of to a secure landfill site operated by the Company.

- Reg. 7.24: Materials coming onto the site appear to be labelled in accordance with the Australian Dangerous Goods Code. However, there appears to be some variability in practices attached to the use of labels when transferring chemicals from supplier-provided containers to smaller, site-issued containers.

- Reg. 7.25: MSDS registers are well developed and maintained. MSDS information is freely accessible to employees and the register contains details of risk assessments carried out in accordance with the regulations.

- Reg. 7.26: Work procedures, rules and training are applied to ensure that persons working in enclosed spaces are aware of the potential risks attached to the use of hazardous substances in those spaces.

- Reg. 7.27: Qualitative and quantitative risk assessments have been carried out, as appropriate, and are formally documented. 
- Reg. 7.28: The hierarchy of controls is widely understood and deployed on the site. Suitable procedures and arrangements apply to the application of engineering, administrative and work practice controls, and personal protective equipment controls.

- Reg. 7.29: Atmospheric monitoring programs are under the direction of a professional Industrial Hygienist and are highly developed. Most, but not all, exposure levels are consistently below those identified in Worksafe Australia's Exposure Standards.

- Reg 7.30: Health surveillance programs are also highly developed. These are under the direction of an Occupational-Physician. Health assessments are carried out at initial employment and periodically thereafter.

- Reg. 4.24-4.29: The site appears to meet regulatory requirements relating to provision of appropriate equipment, facilities and services for first aid. There is a well-equipped Health Centre staffed by Occupational Health Nurses. After hours cover is provided by trained Emergency Response Officers.

- Reg. 4.13: The site appears to meet all regulatory requirements for training of employees and the maintenance of training records.

\subsubsection{Organisation of Work}

This element was examined with the aid of Booklet 4 (Appendix 7). Twenty-two lines of inquiry were pursued under the principal headings of leadership, commitment, planning, organisation, training, communication and measurement. Table 5 below provides a summary of the response from Facility One to the questions. raised in the booklet. Self-ratings fall into four categories - Poor, Fair, Good or Excellent (abbreviated "Exc." in the tables that follow). The interviewee rated nineteen of the twenty-two elements as "Good" or "Excellent", thereby activating follow-up questions about key initiatives and success factors. Elements allocated a "Fair" or "Poor" rating were not pursued any further. 


No. Element $\begin{gathered}\text { Self- Key OHS Initiatives and Success Factors } \\ \text { Rating }\end{gathered}$

1. Management visibility

2. Policy enunciation

3. Tracking performance

4. Financial resources

5. Internal resources

6. External resources

7. Employee involvement

8. Strategic planning
Exc. DuPont-style management safety contacts (formalised and measured). Senior management leadership is evident at safety meetings.

Good Wall-mounted policy statements found in many well-accessed plant locations.

Policy statements feature in many of the location's publications and on various Intranet sites.

Good A Safety Dashboard approach is employed in conjunction with a Balanced Scorecard. Objectives are set (30 day, 60 day, 90 day and annual) and progress to completion is monitored closely.

Good Departmental budgets carry provision for local OHS initiatives (eg. training, equipment, engineering and capital works).

Good Strong medical surveillance and $\mathrm{IH}$ programs are deployed in support of the chemical safety effort.

\section{Fair}

Good There is a high level of employee involvement in Business Centre, departmental and crew level safety activities.

Good A high level of attention is applied to strategic planning for OHS. The A3 process is applied to planning at all levels. 
Table 5

Organisation of Work - Facility One (Continued)

\begin{tabular}{ccc} 
No. Element & $\begin{array}{c}\text { Self- } \\
\text { Rating }\end{array}$ & Key OHS Initiatives and Success \\
& Factors \\
\hline
\end{tabular}

9. Target-setting

10. Contractor plans

11. Organisational structure

12. OHS integration

13. Departmental integration

14. Induction training

15. Refresher training

16. Safety representative training
Good Targets are set on 30-day, 60-day, 90day and annual bases.

Good The Contractor Safety Manual is a principal source of guidance on expectations and performance.

Contractor plans are reviewed as part of pre-qualification checks.

Contractor plans are integrated with those of the site.

Good The organisational structure encourages interaction of $\mathrm{OHS}$ professionals with line management and shop floor personnel.

Good Site and departmental OHS plans are integrated with broader Business Unit plans.

Good Safety consultants and health professionals are assigned to support nominated Business Centres.

Good Induction is structured around a generic, one-day program provided by the Mining and Resource Contractors Safety Training Association (MARCSTA), followed by a one-day site program and a half-day departmental program.

Fair

Good The introductory course is followed by skills training and awareness-building. 
Table 5

Organisation of Work - Facility One (Continued)

No. Element $\begin{gathered}\text { Self- Key OHS Initiatives and Success Factors } \\ \text { Rating }\end{gathered}$

17. Effectiveness of

Fair

training

18. Hazard

communication

Good Many approaches are used in this area eg. Safety Alerts, feedback after air sampling exercises, use of the Chemweb MSDS database, awareness training and similar.

19. Communication of

Good The A3 planning template is used to targets identify problem areas, to define the present state and future state and actions needed to achieve the desired outcomes, and as a means of communication.

20. Delivery of feedback Good There are many channels for feedback from "toolbox" meetings at the shop floor level through to electronic and hard copy reports, newsletters and other presentations.

21. Use of lagging indicators

Good The system is centred on the reporting of lost time injuries, restricted injuries, medical treatments and first aid injuries. This is well deployed and understood.

22. Use of leading Good Quite well developed measures are in indicators place (eg. "safety dashboard" items that relate to provision of training, the number of observations carried out, surveys relating to the use of personal protective equipment (PPE), reductions in the magnitude of exposure or in the number of individuals exposed, and similar). 


\subsubsection{Hazard Recognition}

This element was examined with the aid of Booklet 5 (Appendix 7). Three lines of inquiry were pursued via questions on walk-through surveys (an established technique for identifying hazards, evaluating work practices and checking the efficacy of existing controls), job dictionaries (that itemise jobs and tasks, as a preliminary to assignment of exposure information and the assessment of risk) and chemical inventories. Table 6 below provides a summary of the response from Facility One to the questions raised in the booklet. Self-rating categories are as described earlier. The interviewee rated all three of the sub-elements as "Good" or "Excellent", thereby activating follow-up questions about key initiatives and success factors.

Table 6

Hazard Recognition - Facility One

\section{Self- Key OHS Initiatives and Success}

No. Element Rating Factors

1. Walk-through surveys Good The Site Hygienist conducts these, albeit on an informal basis.

Departmental inventories are checked against chemicals found. Workforce stratified into Similar Exposure Groups (SEGs) - by process, job, task and environmental agent.

2. Job dictionaries

3. Chemical inventories
Good Departmental inventories are in place and used in training, awareness-building and auditing applications. 


\subsubsection{Risk Assessment}

This element was also examined with the aid of Booklet 5 (Appendix 7). Six lines of inquiry were pursued via questions on the existence and effectiveness of sampling plans and schedules, qualitative assessment programs, quantitative assessment programs, statistical treatment of sampling results, exposure baselines and record-keeping. Table 7 below provides a summary of the response from Facility One to the questions raised in the booklet. Self-rating categories are as described earlier. The interviewee rated all six of the sub-elements as "Good", thereby activating follow-up questions about key initiatives and success factors.

\subsubsection{Risk Control}

This element was also examined with the aid of Booklet 5 (Appendix 7). Six lines of inquiry were pursued via questions on processes for feasibility assessment and priority setting (for OHS controls), together with the effectiveness of substitution/elimination activity, procurement controls, engineering controls, administrative/work practice controls and personal protective equipment controls. Table 8 below provides a summary of the response from Facility One to the questions raised in the booklet. Selfrating categories are as described eartier. The interviewee rated all six of the sub-elements as "Good", thereby activating follow-up questions about key initiatives and success factors. 


\begin{tabular}{ccc}
\hline No. Element & $\begin{array}{c}\text { Self- } \\
\text { Rating }\end{array}$ & Key OHS Initiatives and Success \\
& Factors \\
\hline
\end{tabular}

1. Sampling plans

Good The in-house "Hygenius" software package is used to develop annual sampling plans.

2. Qualitative assessments

Good These are carried out with the assistance of the Hygenius v2 software package.

3. Quantitative assessments

Good These are carried out with the assistance of the Hygenius v2 software package.

4. Statistical treatment

5. Exposure baselines

6 Record-keeping
Good Hygenius has the capability to meet all statistical needs (development of geometric means, arithmetic means, geometric standard deviation, and similar).

Good Exposure baselines have been developed for all Similar Exposure Groups on site.

Good The Hygenius products are designed to accommodate longterm storage of electronic records. Historical, hard copy records are stored in secure, offsite archives. 
Table 8

Risk Control - Facility One

Self-

No. Element Rating Key OHS Initiatives and Success

Factors

1. Feasibility assessments Good A formal process is deployed to rank projects in terms of their importance and in terms of the feasibility of achieving a successful outcome.

2. Elimination/substitution Good There are several current examples of successful elimination and substitution effort. The hierarchy of controls is generally well understood at the site and is pursued where feasible.

3. Procurement controls Good $\mathrm{IH}$ and Supply personnel interact formally when vetting new chemicals proposed for use on the site.

4. Engineering controls Good A "Blitz" process is applied to engineering projects. This ensures that a wide variety of inputs are obtained.

There are many examples of successful engineering control on the site.

5. Work practice controls Good The Company's Performance Support System has a library of controlled documents covering hundreds of procedures and administrative arrangements. These are supported by supervisory practices, together with training and awareness packages.

6. PPE controls

Good There is ready availability and deployment of PPE conforming to Australian Standards, and meeting comfort and practicability criteria. 


\subsubsection{Industrial Hygiene Program Development}

This element was also examined with the aid of Booklet 5 (Appendix 7). Eleven characteristics accompany a Stage I (beginning) program, thirteen are used to describe a Stage II (improving) program and twenty are used to describe a Stage III (advanced) program.

Based on the criteria used in Booklet 5, Facility One has an "advanced" industrial hygiene program, characterised by the following features:

- The $\mathrm{IH}$ program is very visible, and there is wide involvement and ownership by stakeholder groups.

- Policies have been endorsed by management and are effectively supported, communicated and deployed.

- Hygiene-specific items feature in the business plans for the facility.

- Roles and accountabilities are clearly established.

- Goals, objectives and measurement criteria are established for each program element.

- A performance evaluation system is applied to the achievement of goals and objectives by line managers.

- Job dictionaries and exposure baselines have been fully developed.

- Chemical inventories and hazard communication programs are well developed.

- Line management understands and applies the hierarchy of controls

- Employees avoid identified health hazards and use the protective devices as supplied.

- Expectations for behaviour have been well developed via rules and procedures.

- The effectiveness of engineering controls is measured at installation and periodically thereafter (eg by re-evaluatisi of employee exposure).

- Records are kept of maintenance, inspection and operation of control devices.

- Formal arrangements in place for contractors to provide information on hazardous materials and equipment they intend to utilise on-site.

- Formal programs are in place for induction and refresher training. 
- Advanced software is used to assist with sample scheduling, statistical treatment of data, the establishment of exposure baselines and the generation of reports.

- IH professionals are extensively networked to government agencies, industry bodies, universities and other sources of expertise.

- Programs are directed by, or guided by, experienced hygienists certified for comprehensive practice of Industrial Hygiene by the American Board of Industrial Hygiene or an equivalent professional organisation.

- Programs frequently exceed the requirements set by Government.

- Arrangements are in place to securely archive all key facility records in relation to Industrial Hygiene.

\subsubsection{People Initiatives}

This element was examined with the aid of Booklet 6 (Appendix 7). Eight lines of inquiry were pursued via questions on the existence and effectiveness of behavioural safety programs, task observation programs (a component within a broader behavioural safety program), motivation and recognition programs, attitude surveys, morale surveys, safety (management) contact programs, compliance surveys relating to the use of personal protective equipment and site promotional efforts for OHS. Table 9 below provides a summary of the response from Facility One to the questions raised in the booklet. Self-rating categories are as described earlier. The interviewee rated four of the eight sub-elements as "Good", thereby activating follow-up questions about key initiatives and success factors attached to these. 
Table 9

People Initiatives - Facility One

No.

Element

Self-

Key OHS Initiatives and Success

Rating

Factors

1. Behavioural safety

Fair

2. Task observation

Fair

3. Motivation/recognition

Good Safety, motivation and recognition team (SMART) activities (employee-led) widely promoted and accepted on site.

4. Attitude surveys

Fair

5. Morale surveys

Fair

6. Management contact

Good

A DuPont-style safety management contact program is formalised, with clear expectations of line management and formal measurement of activity.

7. PPE compliance

Good Compliance surveys.

Self-audit system (ASATs) in place.

Linked to behavioural safety, management contact, supervision, procedures and training initiatives.

8. Promotional efforts

Good Safety Summit series (several topics).

A "Safety Expo" approach.

Front gate displays.

Canteen displays on theme topics. 


\subsubsection{Summary}

The results for Facility One may be represented as a set of scores, developed in accordance with the description provided in Section 3.5. These were:

- The overall score for the compliance element was $95 \%$.

- The overall score for the organisation of work (or management enabler) element was $86 \%$.

- The overall score for the workplace environment (or technical initiatives) element was $100 \%$.

- The overall score for the people initiatives element was $50 \%$.

Industrial Hygiene program development was characterised as "advanced" and assigned a score of $100 \%$. The latter was based on the observation that all program elements identified in Booklet 5 appear to be in place.

38 potential benchmarking opportunities emerged from the process applied to Facility One in this study. 19 of these relate to organisation of work (management enablers), 15 to the workplace environment (technical initiatives) and the remaining four to people initiatives.

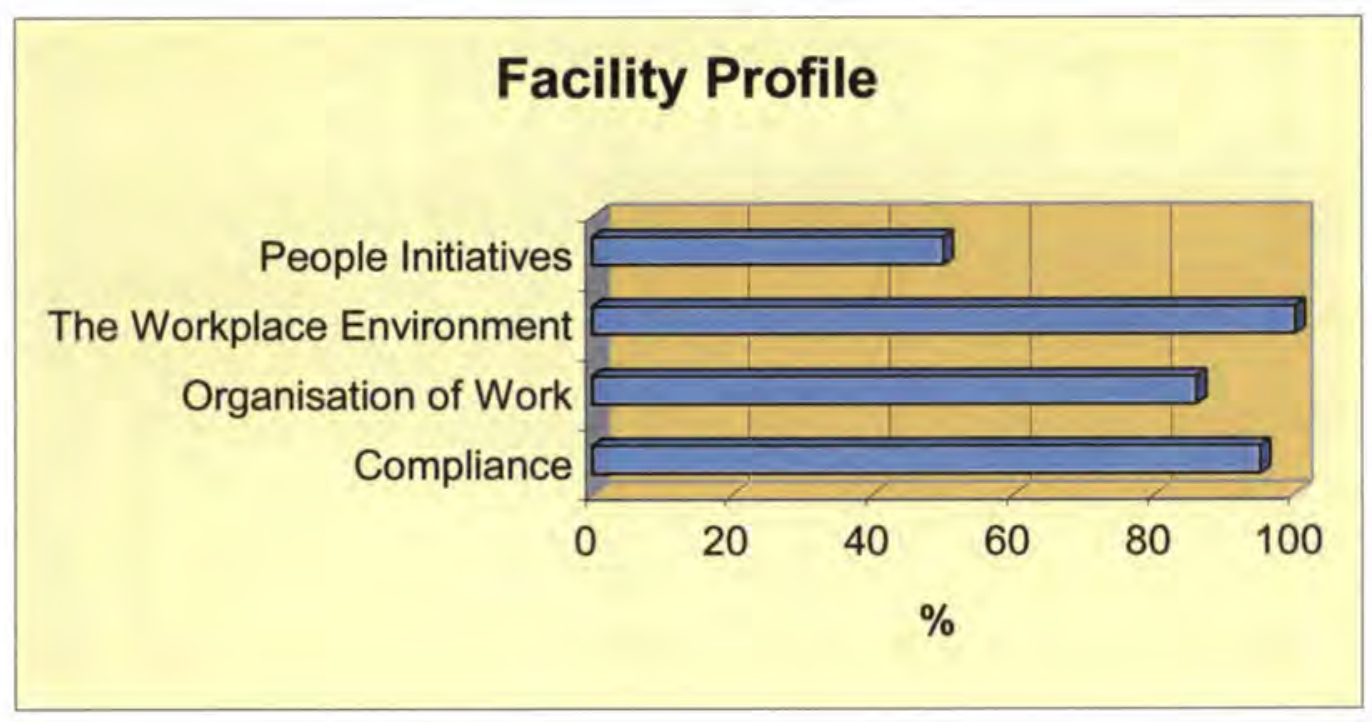

Figure 14: Facility profile - Facility One 


\subsection{Facility Two}

\subsubsection{The Operation}

Facility Two was a bauxite mine located in the Darling Ranges. It belongs to the same organisation that operates the refinery described in the previous section. The facility is in two parts. There is a production area where mobile equipment is located and this includes scrapers, trucks, graders, loaders and similar items. Maintenance of this equipment takes place in a large workshop in a separate part of the mine. There is a smaller workshop located at the production site - used mainly for vehicle servicing. Product from the mine (bauxite) is transported by conveyor belt to the organisation's alumina refinery some 20 kilometres away.

A simplified description of the process is provided in Figure 15.

\subsubsection{The Workforce}

The workforce numbers 220 full-time, permanent employees and 110 fulltime temporary staff. Company employees are split between 50 in the administration and management group, and 170 wages employees in production and maintenance jobs. Approximately half of the workforce is on a rotating shift pattem ( 12 hour shifts or 10.3 hour shifts). There are 20 contracting organisations on-site - mostly engaged in activities such as cleaning, road construction, mobile equipment maintenance, hydrocarbon removal, haul road watering and supply of materials.

The location has a very low rate of labour turnover and there are many employees with more than 10 years of service at the site. 

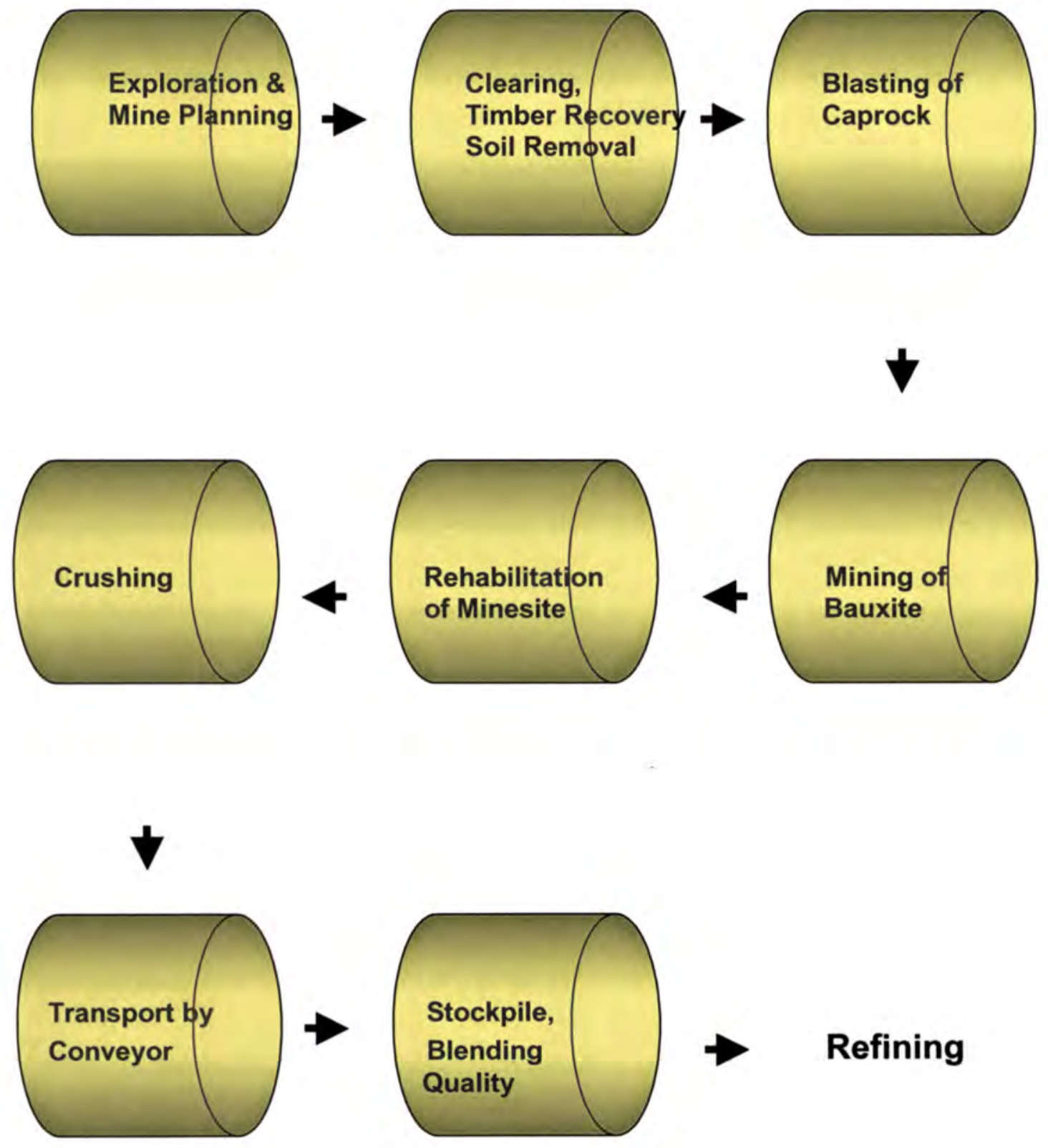

$\rightarrow \quad$ Refining

Figure 15: Schematic flowsheet - bauxite mining process (Facility Two)

\subsubsection{Organisation for $\mathrm{OHS}$}

The location has access to two Occupational Health Nurses from the adjacent refinery. These nurses are employed on a full-time basis, but also have responsibility for the refinery workforce of 650 personnel. The Minesite has access to a further occupational health resource - a 
Physiotherapist/Ergonomist. She works under a shared arrangemerit with another mine site within the same organisation and operates within the Security guardhouse two days per week. An Industrial Hygienist is also available to the location. She is located at the nearby refinery and has responsibilities at that site, as would be expected. There is one full-time professional safety resource person - with responsibility for both mines. The personnel referred to above are supported in their efforts by a safety rerresentative system (about 15 on site) - drawn from the ranks of each natural work group. The organisation has a strong commitment to selfauditing processes and there is an audit coordinator within the Mining Group. He is responsible for the conduct of regular self-audits and for tracking corrective actions that arise from these. The underpinning philosophy at the site, however, is that safety is a "line" responsibility and that Health and Safety Professionals are there to act as a resource only.

Mine site personnel do have problems with accessibility to the above resources - due to their wide areas of responsibility.

\subsubsection{Principal OHS Issues and Challenges}

- Physical hazards:

Production - Noise, vibration, ionising and non-ionising radiation Maintenance - Noise, vibration, thermal stress and non-ionising radiation.

- Chemical hazards:

Production - Bauxite dust, various fuels.

Maintenance - Bauxite dust, welding fume, industrial chemicals (cleaning agents, oils and greases, surfactants, adhesives, sealants, solvents, and similar). The Maintenance Group has a higher potential for exposure to chemicals and so this is where most focus is applied.

- Biological hazards:

None identified.

- Ergonomic hazards:

Production - Seating issues, vibration and access/egress from machinery. 
Maintenance - Pushing, pulling, manual handling and awkward postures.

\subsubsection{Other Relevant Information}

The facility belongs to a global metal business that has a high level of vertical integration, that is, the broader organisation is active in all major segments of the industry: mining, refining, smelting, fabricating and recycling. As such, it obtains leverage and benefits from the OHS knowledge, systems, procedures, tools and resources that reside within the wider organisation.

The Department of Minerals and Petroleum Resources conducted an audit of management systems at Facility Two in July 1999. The site obtained a score of $86 \%$ - based on the structure described by DMPR (2002) and alluded to earlier. Figures provided by the State Mining Engineer ( $M$. Knee, personal communication, March 14, 2003) indicate that 128 mine sites, mineral processing and transporthandling operations in Western Australia have been audited in this way over the past five years. Scores ranged from $35 \%$ to $99 \%$, with a mean of $76 \%$.

In 2002, the site experienced a zero Lost Time Injury Frequency Rate.

\subsubsection{Organisational Effectiveness}

The questions posed in Booklet 2, under the heading "general OHS", are designed to provide a preliminary gauge of organisational effectiveness. The location representative responded in the affirmative to all nine questions. Thus, the facility has in place:

- A clearly expressed OHS policy.

- A philosophy and commitment to exceed minimum standards.

- Integration of OHS into the key business functions of planning, organising, leading, controlling and evaluating.

- Clearly defined OHS performance expectations and measures for all levels in the organisation.

- Injury management plans. 
- Clear evidence of management involvement and commitment to OHS.

- Clearly assigned OHS responsibilities for managers and supervisors, expressed in terms of their normal duties.

- Commitment and involvement of employees, with formal and informal structures to encourage their participation.

- A generally held view within management that Corporate investment in OHS is good business practice.

\subsubsection{Benchmarking, Best Practice and Technology Transfer}

The location representative indicated that there had been very little external OHS benchmarking in the past. Previous efforts have been very specific in nature, rather than looking at broader aspects. Technology transfer was actively encouraged within the wider organisation. However, the location representative noted that interactions on certain types of noisy hand tools had not been beneficial. She considered that this was probably due to noise measurement differences between countries.

\subsubsection{Self-Ranking in OHS}

The location representative thought that her site would rate in the top five percent of sites within the Mining Industry of Western Australia. She based this, in part, on her knowledge of systems and procedures in place, but mainly because of input from networking with other $\mathrm{OHS}$ professionals in the Industry.

\subsubsection{Potential Benchmarking Partners}

The location, as part of the wider organisation, admires the strengths of the DuPont organisation in terms of performance and reputation for excellence in OHS. DuPont representatives have been used in the past to audit the facility in certain areas of OHS performance. Also, on occasions, the site has interacted with other mining facilities in Western Australia to benchmark specific issues, such as service truck design. 


\subsubsection{Chemical Safety Preliminary Questionnaire}

In relation to the chemical safety questionnaire presented in Part B of Booklet 2, the location representative responded to all elements in the affirmative. Thus, the facility is characterised by the following:

- A published policy on the management of hazardous materials.

- Roles and responsibilities have been clearly assigned.

- A current hazardous materials inventory is available.

- Material Saíety Data Sheets (MSDSs) are available for every chemical entering or leaving the site. On rare occasions, a chemical is brought onto site without the correct approval and therefore does not have an MSDS.

- An MSDS will normally accompany the first supply of a hazardous material to the location. There is sometimes an issue with the use of credit cards, phone/faxes or petty cash being used for local purchases, particularly on weekends when access for immediate approval is more difficult.

- There is interaction between the Purchasing and Safety/Health groups to screen new materials requested for purchase.

- There is a system to prevent non-approved chemicals coming on-site and for ensuring that those chemicals that are on-site have been approved. However, as indicated above, there is sometimes an issue with the use of credit cards, phone/faxes and petty cash purchases.

- There is a system, via Purchasing and Stores to track where and how much chemical usage occurs on-site.

- The site complies with all relevant legal requirements for the safe storage, transport, issue, use and disposal of hazardous materials employed or generated by the on-site operations.

- Labels and warning signs are posted in relation to hazardous materials used on-site or sent elsewhere from the site.

- Contractors are expected to meet similar requirements and standards with respect to the hazardous materials they bring onto the site. 


\subsubsection{Flow Chart}

There are many controls built into the process for procurement of hazardous substances. These cover access through the Stores requisition system, by purchase requisition and by direct ordering through a Supply (CLIPS) catalogue of approved hazardous materials. Purchases by petty cash or credit card, or use of free samples are not available without authorisation by the Supply Department or the Site Industrial Hygienist.

Figure 16 represents a simplified version of the steps used at Facility Two to vet and control access of new chemicals to the site.

\subsubsection{Critical Success Factors}

The site representative was asked to nominate the four most critical success factors in relation to chemical safety at the location. She nominated:

- Clearly defined roles and responsibilities regarding the purchase, use and disposal of hazardous materials.

- Interaction between the Industrial Hygienist and the Supply Department.

- Leadership and support from all management levels in the organisation.

- Training and communication. 


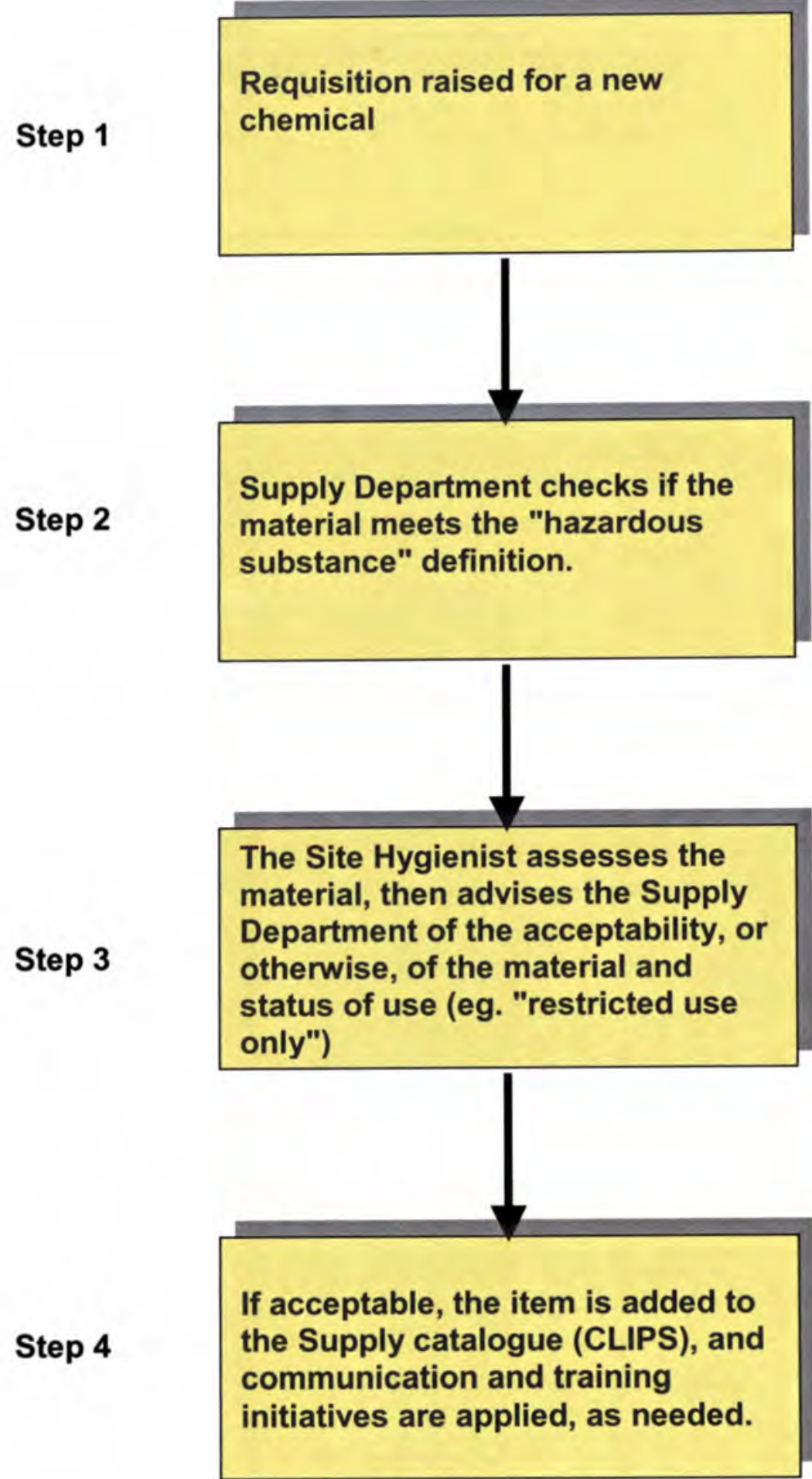

Figure 16: Acquisition of new chemicals - Facility Two

\subsubsection{Performance Measurement}

The traditional lagging indicators of OHS performance are quite prominent at the facility. There is a close focus on Lost Time Injury Frequency Rate 
(LTIFR) - a measure used widely by Government and Industry organisations throughout the country. Also, an internal measure, referred to as "Total Recordable Injury Frequency kate (TRIFR), is used to track all injuries more serious than a first-aid injury. This covers injuries that require some form of medical attention beyond first-aid treatment, injuries that restrict the individual from performing all elements of the job and lost time injuries. The ratio of injury-free events to the all-injury count is used to encourage open reporting of near-hit events or situations that pose a hazard and require some form of corrective action.

Leading indicators of performance have been applied in several areas of OHS. The Department of Minerals and Petroleum Resources conducts regular audits of Dangerous Goods facilities at the site. Additionally, supervisor/foreman safety contacts are deployed on a regular basis. These are highly visible examples of leadership and commitment, and are formalised, measured and reported on a regular basis. They constitute an element of the performance appraisal structure for supervisory personnel. Additionally, there is an activity referred to as GOLF audits (Go, Obsene, Learn, Fix). These are audits of behaviour and are supported and deployed by shop-floor employees. They are also subject to formalised measurement. Such measures reflect compliance with procedures and rules, and are part of bigger behavioural safety efforts at the site.

Chemical exposures are managed and measured via a system that has people in similar exposure groups (SEGs), as described by Mulhausen and Damiano (1998). Corporate targets are set for the reduction in number or magnitude of unacceptable chemical SEGs and progress is tracked on a monthly basis. Once again, the term "unacceptable" is applied when $5 \%$ or more of the sample results are above the occupational exposure limit and is therefore very conservative (protective of health).

A Safety Performance Matrix, similar in format to that developed originally by the Eastman Kodak Company, and described earlier, is deployed by 
the broader organisation as a means of tracking progress with a collection of several leading and lagging indicators.

\subsubsection{Innovations in Chemical Safety}

The location was able to demonstrate many innovations that could be of potential interest to other organisations.

Details are provided in the sections that follow but, in brief, these cover:

- The use of self-assessment audits.

- A template for the approval of hazardous materials (for use on-site).

- Well-deployed procedures for vetting new chemicals and tracking their use and consumption rate on-site.

- Job dictionaries and similar exposure groups (SEGs).

- Qualitative exposure assessments, supported by Hygenius software.

- Quantitative exposure assessments, supported by Hygenius software.

- Measurement and reporting of performance in chemical safety.

- Risk mitigation initiatives.

- Use of unacceptabie exposure plans.

- Medical surveillance.

\subsubsection{Compliance}

This element was examined with the aid of Booklet 3 (Appendix 7 ) and the Western Australian Mines Safety and Inspection Regulations 1995.

- Reg. 7.21: The Material Safety Data Sheets (MSDSs) held on site are in the Worksafe Australia format and are current (by definition, less than 5 years old). An external organisation, Chemwatch, has a contract to supply the MSDSs and part of their obligation is to ensure currency of the sheets. Additionally, there is a compilation of original, hard copy MSDSs from suppliers - covering past and present use of materials on the site.

- Reg. 7.22: Containers are generally of suitable design and manufacture. There was some variability around the site in terms of the practices surrounding the transfer of bulk supplies to more suitable 
smaller containers. The need for decanting is reduced or eliminated by purchasing materials in smaller quantities where applicable/possible.

- Reg. 7.23: Many of the more substantial containers that are used on site are returned to the supplier and are then recycled. The waste disposal contractor removes some materials for specialised disposal at their main laboratory.

- Reg. 7.24: Materials coming onto the site appear to be labelled in accordance with the Australian Dangerous Goods Code. However, there appears to be some variability in practices attached to the use of labels when transferring chemicals from supplier-provided containers to smaller, site-issued containers.

- Reg. 7.25: MSDS registers are well developed and maintained. MSDS information is freely accessible to employees and the register contains details of risk assessments carried out in accordance with the regulations. There are some minor discrepancies with respect to missing MSDSs, but these are being rectified. Employees can access MSDSs via the mainframe system. There is a back-up copy on the Security computer to cover a situation involving failure of the Local Area Network (LAN). If the employee is computer illiterate, assistance is available from several sources.

- Reg. 7.26: Work procedures, rules and training are applied to ensure that persons working in enclosed spaces are aware of the potential risks attached to the use of hazardous substances in those spaces. There is a classification system applied to confined spaces and certain rules go with this. Anyone entering a confined space is required to undergo training on an annual basis, or prior to entry. The work group completes a risk assessment prior to each entry into the confined space.

- Reg. 7.27: Qualitative and quantitative risk assessments have been carried out, as appropriate, and are formally documented.

- Reg. 7.28: The hierarchy of controls is widely understood and deployed on the site. Suitable procedures and arrangements apply to the application of engineering, administrative and work practice controls, and personal protective equipment controls. Some work is 
required with respect to the maintenance of non-disposable respirators, notwithstanding that these represent only a small proportion of respirators used on site (most respirators are of the disposable type). This is reinforced at training sessions and reviewed at audits.

- Reg. 7.29: Atmospheric monitoring programs are under the direction of a professional Industrial Hygienist and are highly developed. Most, but not all, exposure levels are consistently below those identified in Worksafe Australia's Exposure Standards. The Industrial Hygiene Laboratory is NATA accredited, as are external technical $r . . .$. es, wherever possible. In the event of an exposure being above the standard, there is a process whereby an Accident/Incident report form is raised and an investigation is performed to determine the cause. In rare instances where an exposure is consistently above the standard, a Feasibility Assessment is carried out and an Unacceptable Exposure Plan is developed.

- Reg. 7.30: Health surveillance programs are also highly developed. These are carried out at the Health Centre of the nearby refinery and are under the direction of an Occupational Physician. Health assessments are carried out at initial employment and periodically thereafter.

- Reg. 4.24-4.29: The site appears to meet regulatory requirements relating to provision of appropriate equipment, facilities and services for first aid. An ambulance is located near the main Maintenance workshop site. There are two qualified first aid personnel available at the maintenance site and one available at the production site. Each first aider is trained in the use of Oxyviva equipment and the Heart Start machine.

- Reg. 4.13: The site appears to meet all regulatory requirements for training of employees and the maintenance of training records. The Industrial Hygiene Department conducts refresher training on an annual basis. This training includes the use of MSDSs. Training records are kept on a computer program (the Learning Management System) that is maintained by the Training Department. 


\subsubsection{Organisation of Work}

This element was examined with the aid of Booklet 4 (Appendix 7). Twenty-two lines of inquiry were pursued under the principal headings of leadership, commitment, planning, organisation, training, communication and measurement. Table 10 below provides a summary of the response from Facility Two to the questions raised in the booklet. Self-ratings fall into four categories - Poor, Fair, Good or Excellent. The intenviewee rated twenty of the twenty-two elements as "Good" or "Excellent", thereby activating follow-up questions about key initiatives and success factors. Elements allocated a "Fair" rating in the table that follow are usually associated with programs or efforts that are still at an eariy stage of development or are carried out sporadically. 


No. Element $\quad \begin{gathered}\text { Self- Key OHS Initiatives and Success Factors } \\ \text { Rating }\end{gathered}$
.

1. Management visibility

2. Policy enunciation

3. Tracking performance

4. Financial resources

5. Internal resources

6. External resources

7. Employee involvement
Good Management safety contacts are formalised and measured. Line management leadership is evident at safety meetings.

Good Wall-mounted policy statements are found in several locations. Policy statements feature in many of the broader organisation's publications and on various Intranet sites.

Good A Balanced Scorecard approach is employed and performance expectations are established. Objectives are set ( 30 day, 60 day, 90 day and annual) and progress to completion is monitored closely.

Exc. Departmental budgets are developed on an annual basis and carry provision for local OHS initiatives (eg. training, equipment, engineering and capital works).

Fair

Good There is ready access to external providers and Government agencies.

Good There is a high level of employee involvement via departmental and crew level safety activities, including safety meetings and toolbox talks. 


\begin{tabular}{lcc}
\hline No. Element & $\begin{array}{c}\text { Self- } \\
\text { Rating }\end{array}$ & Key OHS Initiatives and Success \\
& Factors \\
\hline
\end{tabular}

\section{Strategic planning}

9. Target-setting

10. Contractor plans

11. Organisational structure

12. OHS integration

13. Departmental integration

14. Induction training

15 Refresher training
Exc. A high level of attention is applied to strategic planning for OHS. A crew matrix is completed and utilised to ensure the plan is achieved.

Exc. Targets are set on 30-day, 60-day, 90-day and annual bases.

Good Contractor plans are reviewed as part of pre-qualification checks. Some elements are integrated with those of the site. Training is tracked via the Learning Management System (LMS)

Exc. The organisational structure encourages interaction of $\mathrm{OHS}$ professionals with line management and shop floor personnel.

Exc. Site and departmental OHS plans are integrated with broader Business Unit plans. Daily meetings are a feature.

Exc. Very good interaction between Supply, Environmental and $\mathrm{IH}$ Departments.

Exc. Induction is structured around a oneday MARCSTA program (generic), followed by a one-day Company program and a half-day departmental program. Details are entered into LMS Good Each crew has a matrix for all required training for the year. Details are entered into LMS. 
No.

Element

Self- Key OHS Initiatives and Success Factors

Rating

16. Safety rep training training

18. Hazard

communication

19. Communication of targets

20. Delivery of feedback

21. Use of lagging indicators

22. Use of leading indicators
Good The introductory Company-run course is followed by further skills training and awareness-building.

Fair

Good Many approaches are used in this area eg. Significant Incident Reports, toolbox talks, Safety Alerts, feedback after air sampling exercises, use of the Chemweb MSDS database, awareness training and similar.

Exc. The A3 planning template is used to identify problem areas, to define the present state and future state and actions needed to achieve the desired outcomes, and as a means of communication. The safety matrix and performance appraisals are further tools for communication.

Good There are many channels for feedback from "toolbox" meetings at the shop floor level through to electronic and hard copy reports, newsletters and other presentations. The safety matrix involves feedback every five weeks.

Exc. The system is centred on the reporting of lost time injuries, restricted injuries, medical treatments and first aid injuries. This is well deployed and understood.

Good Various measures are in place (eg. ASAT audits, GOLF audits and foremen audits). 


\subsubsection{Hazard Recognition}

This element was examined with the aid of Booklet 5 (Appendix 7). Three lines of inquiry were pursued via questions on walk-through surveys (an established technique for identifying hazards, evaluating work practices and checking the efficacy of existing controls), job dictionaries (that itemise jobs and tasks, as a preliminary to assignment of exposure information and the assessment of risk) and chemical inventories. Table 11 below provides a summary of the response from Facility Two to the questions raised in the booklet. Self-rating categories are as described earlier. The interviewee rated all three of the sub-elements as "Good", thereby activating follow-up questions about key initiatives and success factors.

Table 11

Hazard Recognition - Facility Two

\begin{tabular}{lcc}
\hline No. & Self- & Key OHS Initiatives and Success \\
Rating & Factors \\
\hline
\end{tabular}

1. Walk-through surveys Good Foremen do an inspection during

2. Job dictionaries

3. Chemical inventories every shift and results are logged onto a spreadsheet. Some surveys are conducted by the Industrial Hygiene Department, as required. Job dictionaries are established. They are updated on an annual basis.

Good Chemical inventories exist and are updated each year by area personnel. These are used in training, awareness-building and auditing applications. 


\subsubsection{Risk Assessment}

This element was also examined with the aid of Booklet 5 (Appendix 7). Six lines of inquiry were pursued via questions on the existence and effectiveness of sampling plans and schedules, qualitative assessment programs, quantitative assessment programs, statistical treatment of sampling results, exposure baselines and record-keeping. Table 12 below provides a summary of the response from Facility Two to the questions raised in the booklet. Self-rating categories are as described earlier. The interviewee rated all six of the sub-elements as "Good", thereby activating follow-up questions about key initiatives and success factors.

\subsubsection{Risk Control}

This element was also examined with the aid of Booklet 5 (Appendix 7). Six lines of inquiry were pursued via questions on processes for feasibility assessment and priority setting (for OHS controls), together with the effectiveness of substitution/elimination activity, procurement controls, engineering controls, administrative/work practice controls and personal protective equipment controls. Table 13 below provides a summary of the response from Facility Two to the questions raised in the booklet. Selfrating categories are as described earlier. The interviewee rated all six of the sub-elements as "Good", thereby activating follow-up questions about key initiatives and success factors. 
Table12

Risk Assessment - Facility Two

\begin{tabular}{ccc} 
No. Element & $\begin{array}{c}\text { Self- } \\
\text { Rating }\end{array}$ & Key OHS Initiatives and Success \\
\hline
\end{tabular}

1. Sampling plans

2. Qualitative assessments

3. Quantitative assessments

4. Statistical treatment

5. Exposure baselines

6 Record-keeping
Good A sampling plan is assembled at the beginning of each year. Plans are derived from the requirements of the Healthwise epidemiological study, the Government's CONTAM expectations and the in-house "Hygenius" software.

Good These are carried out with the assistance of the Hygenius $Q$ software package.

Good These are carried out with the assistance of the Hygenius software package.

Good Hygenius has the capability to meet all statistical needs (geometric mean, arithmetic mean, geometric standard deviation, and similar).

Good Exposure baselines have been developed for all Similar Exposure Groups on site. Some issues with job/shift change.

Good The Hygenius products are designed to accommodate longterm storage of electronic records. Historical, hard copy records are stored in secure, offsite archives. 
Table 13

Risk Control - Facility Two

\begin{tabular}{|c|c|c|c|}
\hline \multirow{3}{*}{ No. } & \multicolumn{2}{|r|}{ Self- } & \\
\hline & Element & Rating & Key OHS Initiatives and Success \\
\hline & & & Factors \\
\hline
\end{tabular}

1. Feasibility assessments Good A Company template is used to establish priorities and conduct feasibility assessments for engineering controls.

2. Elimination/substitution Good There are several current examples of successful elimination and substitution effort at the site. The hierarchy of controls is well understood and is pursued where feasible.

3. Procurement controls Good $\mathrm{IH}$ and Supply personnel interact formally and informally when vetting new chemicals proposed for use on the site.

4. Engineering controls

Good A "Blitz" process is applied to engineering projects. This ensures that a wide variety of inputs are obtained.

5. Work practice controls Good The Company's Performance Support System has a library of controlled documents covering hundreds of procedures and administrative arrangements. These are supported by supervisory practices, together with training and awareness packages.

Procedures are written prior to sign-off on the project.

6. PPE controls

Good There is ready availability and deployment of PPE conforming to Australian Standards, and meeting comfort and practicability criteria. 


\subsubsection{Industrial Hygiene Program Development}

This element was also examined with the aid of Booklet 5 (Appendix 7). Eleven characteristics accompany a Stage I (beginning) program, thirteen are used to describe a Stage II (improving) program and twenty are used to describe a Stage III (advanced) program.

Based on the criteria used in Booklet 5, Facility Two has an "advanced" industrial hygiene program, characterised by the following features:

- The IH program is very visible, and there is wide involvement and ownership by stakeholder groups.

- Policies have been endorsed by management and are effectively supported, communicated and deployed.

- Hygiene-specific items feature in the business plans for the facility.

- Roles and accountabilities are clearfy established.

- Goals, objectives and measurement criteria are established for each program element.

- A performance evaluation system is applied to the achievement of goals and objectives by line managers.

- Job dictionaries and exposure baselines have been fully developed.

- Chemical inventories and hazard communication programs are welf developed.

- Line management understands and applies the hierarchy of controls

- Employees avoid identified health hazards and use the protective devices as supplied.

- Expectations for behaviour have been well developed via rules and procedures.

- The effectiveness of engineering controls is measured at installation and periodically thereafter (eg by re-evaluation of employee exposure).

- Records are kept of maintenance, inspection and operation of control devices.

- Formal arrangements in place for contractors to provide information on hazardous materials and equipment they intend to utilise on-site.

- Formal programs are in place for induction and refresher training. 
- Advanced software is used to assist with sample scheduling, statistical treatment of data, the establishment of exposure baselines and the generation of reports.

- $H$ professionals are extensively networked to government agencies, industry bodies, universities and other sources of expertise.

- Programs are directed by, or guided by, experienced hygienists certified for comprehensive practice of Industrial Hygiene by the American Board of Industrial Hygiene or an equivalent professional organisation.

- Programs frequently exceed the requirements set by Government.

- Arrangements are in place to securely archive all key facility records in relation to Industrial Hygiene.

\subsubsection{People Initiatives}

This element was examined with the aid of Booklet 6 (Appendix 7). Eight lines of inquiry were pursued via questions on the existence and effectiveness of behavioural safety programs, task observation programs (a component within a broader behavioural safety program), motivation and recognition programs, attitude surveys, morale surveys, safety (management) contact programs, compliance surveys relating to the use of personal protective equipment and site promotional efforts for OHS. Table 14 below provides a summary of the response from Facility Two to the questions raised in the booklet. Self-rating categories are as described earlier. The interviewee rated five of the eight sub-elements as "Good", thereby activating follow-up questions about key initiatives and success factors attached to these. 
Table 14

People Initiatives - Facility Two

$\begin{array}{ccc}\text { No. Element } & \begin{array}{c}\text { Self- } \\ \text { Rating }\end{array} & \text { Key OHS Initiatives and } \\ \text { Success Factors }\end{array}$

1. Behavioural safety

2. Task observation

3. Motivation/recognition

4. Attitude surveys

5. Morale surveys

6. Management contact

7. PPE compliance

8. Promotional efforts
Good The MATE program and foreman audits are part of this.

Good A "golf card" (GOLF) innovation is used for this purpose.

Good Safety, motivation and recognition team activities (employee-led) are widely promoted and accepted on site. Under the MATE scheme, nominated individuals receive a small gift and a donation is made to the charity of their choice.

Fair

Fair

Good A weekly management contact program is formalised, with clear expectations of line management and formal measurement of activity.

Good The self-assessment tools (ASATs) provide a good checking mechanism. Linked to behavioural safety, management contact, supervision, procedures and training initiatives.

Fair 


\subsubsection{Summary}

The results for Facility Two provided above may be represented as the following scores:

- The overall score for the compliance element was $95 \%$.

- The overall score for the organisation of work (or management enabler) element was $91 \%$.

- The overall score for the workplace environment (or technical initiatives) element was $100 \%$.

- The overall score for the people initiatives element was $62 \%$.

Industrial Hygiene program development was characterised as "advanced" and assigned a score of $100 \%$. The latter was based on the observation that all program elements identified in Booklet 5 appear to be in place.

Forty potential benchmarking opportunities emerged from the process applied to Facility Two in this study. Twenty of these relate to organisation of work (management enablers), fifteen to the workplace environment (technical initiatives) and the remaining five to people initiatives.

\section{Facility Profile}

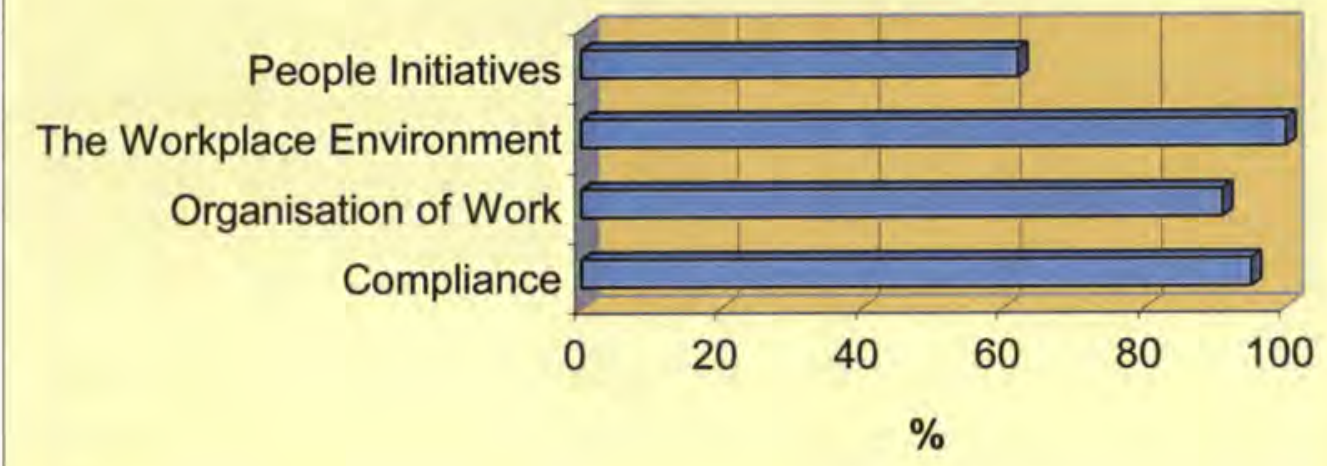

Figure 17: Facility profile - Facility Two 


\subsection{Facility Three}

\subsubsection{The Operation}

Facility Three was a plant involved in the manufacture of a broad range of chemicals for mineral processing and agricultural markets in Western Australia and interstate. In addition, there are tank terminal facilities for bulk liquid storage ano petroleum products, together with transport operations involving the movement of Dangerous Goods to customer facilities.

The main raw materials are sulphuric acid, caustic soda, alumina, copper, ammonia, carbon disulphide, silicate glass and sand. The main end products are copper sulphate, aluminium sulphate, xanthates, sodium aluminate, granulated products, ammonium chloride, sodium silicates and sulphur products. The main waste materials are solid wastes (mainly copper-based salts) and these are disposed of to secure landfill.

A schematic representation of the three principal site functions is provided in Figure 18.

\subsubsection{The Workforce}

The workforce numbers 176 full-time, permanent employees - split between 24 in the administration and management group, 83 in production roles, 46 engaged in maintenance and 23 with transport roles. Shift pattems are variable across different operations within the facility, although they tend to be mainly 8 or 12 hour shifts spread across 6 or 7 days. There are no permanent contractors on site. Contractors are used on an "as needed" basis and numbers may vary from one or two per week up to $10-15$ or more during major projects.

The location has a low rate of labour turnover and there are many employees with more than 10 years of service at the site. 
Manufacturing

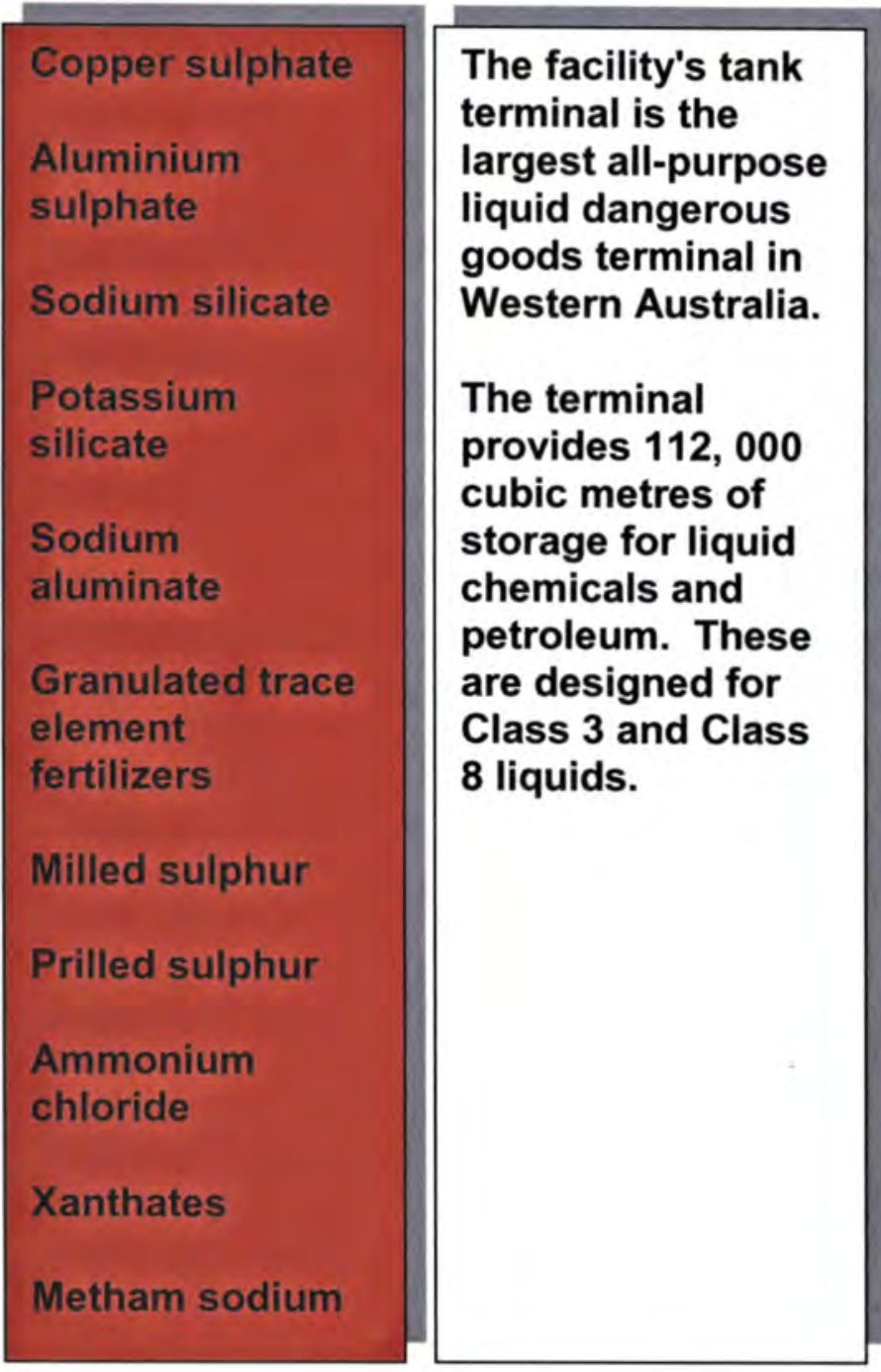

Transport Services

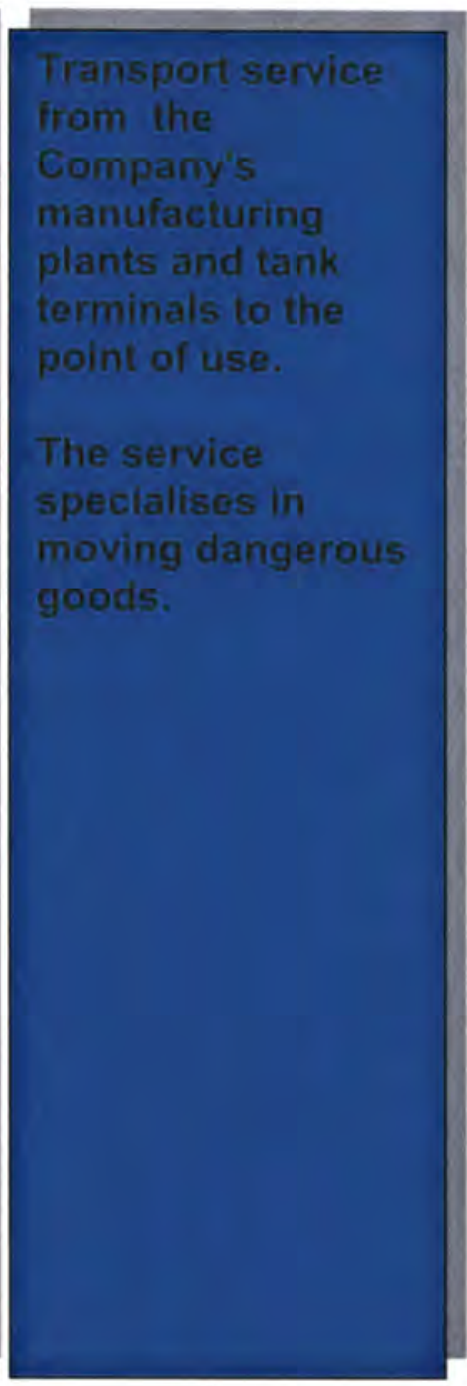

Figure 18: Schematic of operational areas - Facility Three

\subsubsection{Organisation for $\mathrm{OHS}$}

Approximately half of the members of the workforce hold a St John Ambulance Level 1, 2 or 3 certificate - so, the site is well catered for in the first aid area. Arrangements are in place for local, external health care providers to respond to injuries that require higher levels of attention.

There is a HSE\&Q manager who coordinates environment, health, safety and quality activities on the site. A HSE\&Q Coordinator and a HSE\&Q 
Supervisor act in suppcit. These people are aided in their efforts by a plant safety committee structure that involves management and members of the workforce. The underpinning philosophy at the site is that safety is the responsibility of all personnel.

\subsubsection{Principal OHS Issues and Challenges}

- Physical hazards: Steam pipes, liquid outlets, valves, flexible product hoses, reaction pressure vessels, overhead structures, elevated work areas and traffic movement on site.

- Chemical hazards: The principal site chemicals fall into three of the classes nominated in the Australian Dangerous Goods Code, namely Class Three (Flammable Liquids), Class Six (Poisonous and Infectious Substances) and Class Eight (Corrosives). Minor use materials, such as abrasives, cleaning agents, oils and greases, surfactants, adhesives, sealants, solvents, surface coatings and similar, are dealt with as second level items and are covered by a MSDS system. There are about 120 materials on site of which about two-thirds fall into the second category.

- Biological hazards: There are no significant biological hazards.

- Ergonomic hazards: There are manual handling issues associated with finished product packaging and material movements. Lifting, pushing, pulling, twisting, awkward postures, repetitive motion and similar have potential to produce ergonomic injury.

\subsubsection{Other Relevant Information}

The facility has developed a culture of shared responsibility of EHS across the specirum of its workforce. As part of this approach, the Company has implemented an Integrated Risk Management System. Supporting documents were provided to the researcher. These took the form of a Safety, Health \& Environmental Policy and Procedures Manual, Site Layout diagrams and a copy of the facility's organisation chart.

There is a safety incentive program in place. This involves a cash payment to all employees if there are no lost time injuries over a threemonth period. 
The facility sponsors a compliance audit every 12 months. This is carried out by external consultants and has the endorsement and scrutiny of the Department of Industry and Resources of Western Australia. in its latest audit, the site was audited against the 21 elements of its Integrated Risk Management System Manual. These included hazard identification, safe work practices, emergency planning, permit-to-work systems, employee consultation, and similar. Compliance scores for individual elements varied from $70-100 \%$, with a mean score of $92 \%$. In addition to the above, 6-monthly ISO 9000 audits and 12-monthly OHS \& E insurance audits are conducted.

In 2002, the site experienced a zero Lost Time Injury Frequency Rate.

\subsubsection{Organisational Effectiveness}

The questions posed in Booklet 2, under the heading "general OHS", are designed to provide a preliminary gauge of organisational effectiveness. The location representative responded in the affirmative to all nine questions. Thus, the facility has in place:

- A clearly expressed OHS policy.

- A philosophy and commitment to exceed minimum standards.

- Integration of OHS into the key business functions of planning, organising, leading, controlling and evaluating.

- Clearly defined OHS performance expectations and measures for all levels in the organisation.

- Injury management plans.

- Clear evidence of management involvement and commitment to OHS.

- Clearly assigned OHS responsibilities for managers and supervisors, expressed in terms of their normal duties.

- Commitment and involvement of employees, with formal and informal structures to encourage their participation.

- A generally held view within management that Corporate investment in OHS is good business practice. 


\subsubsection{Benchmarking, Best Practice and Technology Transfer}

The location representative indicated that the facility had not previously been involved in a formal OHS benchmarking exercise, structured technology transfer activities in OHS or with any structured activities aimed at identifying best practice with respect to OHS. However, the site does engage in formal OHS audit activity, as described earlier.

\subsubsection{Self-Ranking in OHS}

The location representative thought that his site would rate in the top 25 percent of comparable sites within Western Australian Industry. He based this on his knowledge of systems and procedures in place at his site, together with the low incidence of chemical-related injury and near-miss experience at the facility. He also had some anecdotal information from his external professional network and from suppliers and others who move from site to site.

\subsubsection{Potential Benchmarking Partners}

Three organisations were nominated as "most admired" in terms of their management of OHS. These were the DuPont, Dow and General Electric organisations. Within Australia, the Alcoa and BHP OHS systems were rated highly.

\subsubsection{Chemical Safety Preliminary Questionnaire}

In relation to the chemical safety questionnaire presented in Part $B$ of Booklet 2, the location representative responded to ten of the eleven elements in the affirmative. Thus, the facility is characterised by the following:

- Roles and responsibilities have been clearly assigned.

- A curreni hazardous materials inventory is available.

- Material Safety Data Sheets (MSDSs) are available for every chemical entering or leaving the site.

- An MSDS will normally accompany the first supply of a hazardous material to the location. 
- There is interaction between the Purchasing and Safety/Health groups to screen new materials requested for purchase.

- There is a system to prevent non-approved chemicals coming on-site and for ensuring that those chemicals that are on-site have been approved.

- There is a system to track where and how much chemical usage occurs ori-site.

- The site complies with all relevant legal requirements for the safe storage, transport, issue, use and disposal of hazardous materials employed or generated by the on-site operations.

- Labels and warning signs are posted for every hazardous material used on-site or sent elsewhere from the site.

- Contractors are expected to meet similar requirements and standards with respect to the hazardous materials they bring onto the site.

However, there is no published policy on the management of hazardous materials. The latter falls under the Company's broader policy statements for EHS.

\subsubsection{Flow Chart}

There are several contrcls built into the process for procurement of hazardous substances. These cover access through the Stores requisition system, by purchase requisition and by direct ordering through a Supply catalogue of maintenance and office consumables. Purchases by credit card are restricted to supervisor level personnel, or higher.

Figure 19 represents a simplified version of the steps used at Facility Three to vet and control access of new chemicals to the site. 


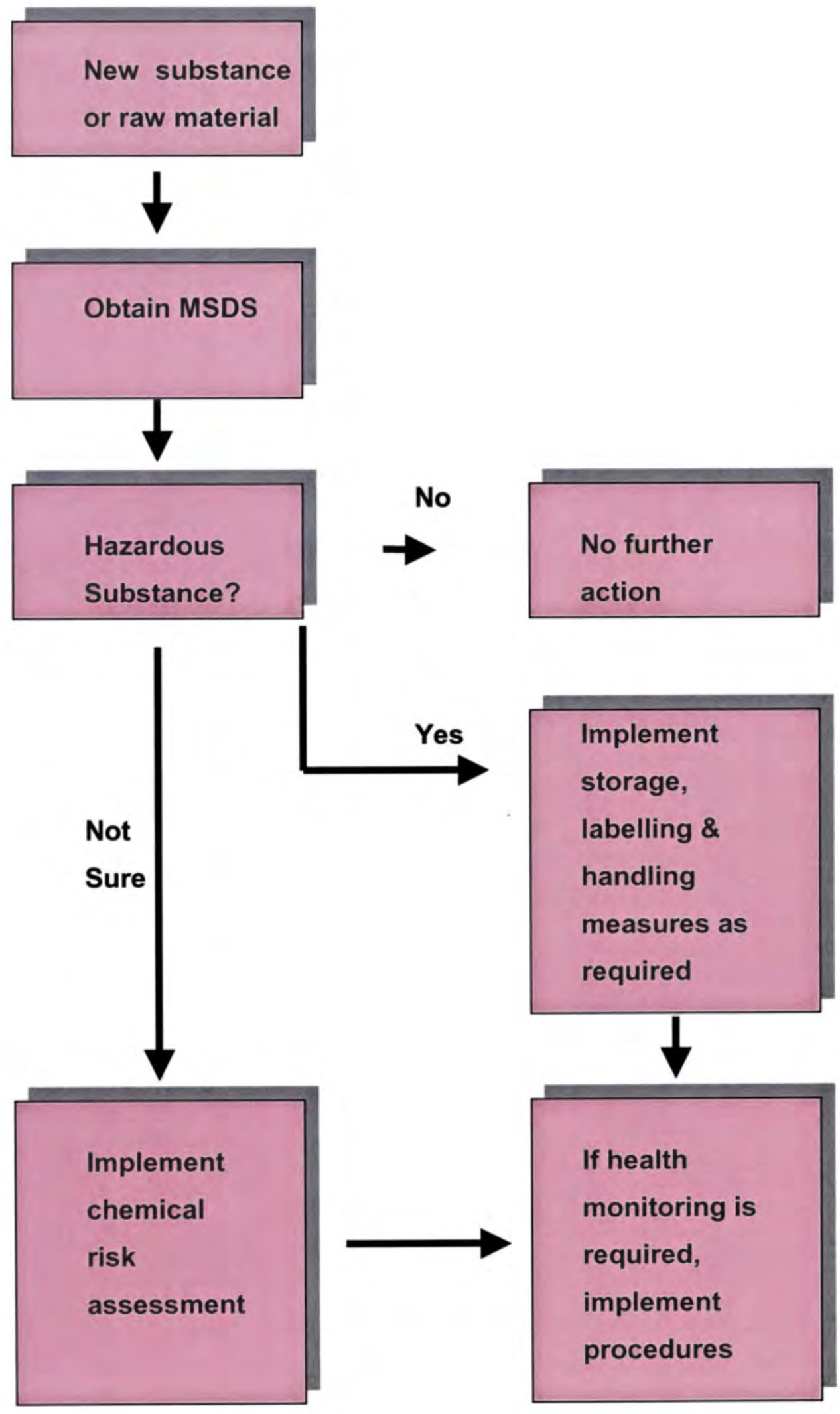

Figure 19: Acquisition of new chemicals - Facility Three 


\subsubsection{Critical Success Factors}

The site representative was asked to nominate the four most critical success factors in relation to chemical safety at the location. He nominated:

- Effective communication of relevant safety issues.

- Structured education and training procedures.

- Employee awareness.

- A strong safety culture.

\subsubsection{Performance Measurement}

The traditional lagging indicators of OHS performance are quite prominent at the facility. There is a close focus on Lost Time Injury Frequency Rate (LTIFR) - a measure used widely by Government and Industry organisations throughout the country. Also, an internal measure, referred to as Medical Treatment Injuries (MTls), is used to track injuries that require more attention than a first aid treatment. Injury-free events (IFEs) are another important measure. These cover near miss situations and hazardous situations where action is needed to reduce the level of risk. Reporting of IFEs is facilitated by the ready availability of computers - on average, there is one computer for every two employees. Nonconformance reports and housekeeping checks are also monitored.

Leading indicators of performance have been applied in several areas the number of HSE audits completed, close out or finalisation of audit. recommendations and HSE surveys and questionnaires.

\subsubsection{Innovations in Chemical Safet;}

The facility has developed a series of in-house Computer Based Training (CBT) programs for its most common and high-risk hazardous substances. The CBT currently includes packages for sulphuric acid and caustic soda, but is being further developed to include a range of other materials. Risk assessments tave been carried out on all significant materials and records are kept on these. 


\subsubsection{Compliance}

This facility is different from the other participating sites in that its occupational health and safety activities are covered by Worksafe Western Australia, while its Dangerous Goods activities are under the auspices of the Department of Industry and Resources (formerly, the Department of Minerals and Petroleum Resources). Since the regulatory requirements relating to hazardous substances are virtually identical for mining vs nonmining sites, it was decided, in the interests of consistency, to persevere with use of Booklet 3 (Appendix 7) and the Westem Australian Mines Safety and inspection Regulations 1995 for this application.

- Reg. 7.21: The Material Safety Data Sheets (MSDSs) held on site are in the Worksafe Australia format and are current (by definition, less than 5 years old). These are sourced from an external provider, Chem Alert, and a contract is in place to give effect to this service. There is no archiving of MSDSs that relate to superseded materials or earlier formulations.

- Reg. 7.22: Containers are generally of suitable design and manufacture and meet the requirements of the Australian Dangerous Goods Code.

- Reg. 7.23: Some containers that are used on site are returned to the supplier. Others are disposed of to a Government-controlled, secure landfill site.

- Reg. 7.24: Materials coming onto the site (and exiting from the site) appear to be labelled in accordance with the Australian Dangerous Goods Corde. There is little need to decant into smaller containers for on-site usi.

- Reg. 7.25: MSDS registers are well developed and maintained. MSDS information is freely accessible to employees and the register contains details of risk assessments carried out in accordance with the regulations.

- Reg. 7.26: Work procedures, rules and training are applied to ensure that persons working in enclosed spaces are aware of the potential risks attached to the use of hazardous substances in those spaces. 
- Reg. 7.27: Qualitative and quantitative risk assessments have been carried out, as appropriate, and are formally documented. A copy of the site Hazard Register was sighted.

- Reg. 7.28: The hierarchy of controls is well understood and deployed on the site. Suitable procedures and arrangements apply to the application of engineering, administrative and work practice controls, and personal protective equipment controls.

- Reg. 7.29: Atmospheric monitoring programs are under the direction of the HSE\&Q Manager. Efforts have been directed at high-risk contaminants such as carbon disulphide, acid mist, copper sulphate dust and ammonium chloride mist. Most exposure levels are consistently below those identified in Worksafe Australia's Exposure Standards.

- Reg. 7.30: Health surveillance programs well developed. These are under the direction of the HSE\&Q Manager. Health assessments are carried out at initial employment and periodically thereafter. Employee health checks are provided on a voluntary basis and are available annually.

- Reg. 4.24-4.29: The site appears to meet regulatory requirements relating to provision of appropriate equipment, facilities and services for first aid. There are three first aid stations on the site.

- Reg. 4.13: The site appears to meet all regulatory requirements for training of employees and the maintenance of training records.

\subsubsection{Organisation of Work}

This element was examined with the aid of Booklet 4 (Appendix 7). Twenty-two lines of inquiry were pursued under the principal headings of leadership, commitment, planning, organisation, training, communication and measurement. Table 15 below provides a summary of the response from Facility Three to the questions raised in the booklet. Self-ratings fall into four categories - Poor $(P)$, Fair $(F)$, Good $(G)$ or Excellent $(E)$. The interviewee rated thirteen of the twenty-two elements as "Good", thereby activating follow-up questions about key initiatives and success factors. Elements allocated a "Fair" rating in the table that follow are usually 
associated with programs or efforts that are still at an early stage of development or are carried out sporadically. "Poor" was applied in the sense that the element was non-existent at the location. There may be good reasons for that - it may not have been needed, may not be appropriate, or an alternative approach may have been used to achieve the same end point.

Table 15

Organisation of Work - Facility Three

No. Element $\begin{gathered}\text { Self- Key OHS Initiatives and Success Factors } \\ \text { Rating }\end{gathered}$

1. Management visibility

2. Policy enunciation

3. Tracking performance
Good Senior management leadership is evident at safety meetings (employee meetings, toolbox meetings, SHE Committee meetings and at safety recognition functions).

Good HSE policies are clearly displayed on-site and in employee information booklets and HSE manuals. HSE is a central focus of all employee inductions and is often raised for discussion at employee meetings.

Good OHS monitoring and reporting is highly visible through published statistical performance charts, annual IRMS Management Review meetings and OHS auditing and reporting. Performance is reported formally to the Board on an annual basis.
4. Financial resources
Fair
5. Internal resources
Fair 


\begin{tabular}{ccc}
\hline No. Element & $\begin{array}{c}\text { Self- } \\
\text { Rating }\end{array}$ & Key OHS Initiatives and Success \\
& Factors \\
\hline
\end{tabular}

6. External resources

7. Employee involvement

8. Strategic planning

9. Target-setting

10. Contractor plans

11. Organisational structure

12. OHS integration

13. Departmental integration
Good The facility is active within several Industry bodies and draws support from these. Key site personnel have a strong network of contacts and resources.

Good The Company philosophy is to heavily involve employees in the development and deployment of HSE initiatives.

Employees are provided with the tools and training to enable them to take full responsibility for their own HSE performance.

Fair

Fair

Fair

Good The Executive Manager - HSEQ coordinates and develops HSE initiatives. He reports directly to the Managing Director and the Company Board.

Good The Company has developed an "Integrated Risk Management System" which seeks to integrate HSE and QA into an overall approach to risk management.

Good All departments participate in site programs. There is evidence of adequate integration. 


\section{No. Element Self- Key OHS Initiatives and Success Factors \\ Rating}

14. Induction training

Good The Company has a comprehensive site induction program. This is complemented with a computer-based training module, an in-house induction video and a competency questionnaire.

15. Refresher training Fair

16. Safety rep training Fair

17. Effectiveness of Fair training

18. Hazard communication

\section{Good}

Initiatives are built around training programs, risk assessment activities per procedures and booklets, and signage, labels and hazard area diagrams.

19. Communication of targets

Good Communications include publication of monthly OHS statistics, bi-monthly HSE Newsletter, published notices, computerbased reporting systems and delivery via employee work groups and committees.

20. Delivery of feedback Good Per activities mentioned above.

21. Use of lagging Good indicators

22. Use of leading Poor indicators

\subsubsection{Hazard Recognition}

This element was examined with the aid of Booklet 5 (Appendix 7). Three lines of inquiry were pursued via questions on walk-through surveys (an 
established technique for identifying hazards, evaluating work practices and checking the efficacy of existing controls), job dictionaries (that itemise jobs and tasks, as a preliminary to assignment of exposure information and the assessment of risk) and chemical inventories. Table 16 below provides a summary of the response from Facility Three to the questions raised in the booklet. Self-rating categories are as described earlier. None of the sub-elements were nominated as "Good" or "Excellent", thus there were no follow-up questions about key initiatives and success factors.

Table 16

Hazard Recognition - Facility Three

Self- Key OHS Initiatives and Success

No. Element Rating Factors

1. Walk-through surveys Fair

2. Job dictionaries Poor

3. Chemical inventories Fair

\subsubsection{Risk Assessment}

This element was also examined with the aid of Booklet 5 (Appendix 7). Six lines of inquiry were pursued via questions on the existence and effectiveness of sampling plans and schedules, qualitative assessment programs, quantitative assessment programs, statistical treatment of sampling results, exposure baselines and record-keeping. Table 17 below provides a summary of the response from Facility Three to the questions raised in the booklet. Self-rating categories are as described earlier. The interviewee rated one of the sub-elements as "Good" (qualitative exposure assessment), thereby activating follow-up questions about the subject involved (qualitative assessments). The workplace monitoring and 
exposure assessment program is described in the Company's Policy and Procedures Manual (copy supplied).

Table 17

Risk Assessment - Facility Three

\begin{tabular}{ccc}
\hline No. Element & $\begin{array}{c}\text { Self- } \\
\text { Rating }\end{array}$ & Key OHS Initiatives and Success \\
& Factors \\
\hline
\end{tabular}

1. Sampling plans Poor

2. Qualitative assessments Good A detailed program is described in the SHE Policy \& Procedures Manual.
3. Quantitative assessments
Fair
4. Statistical treatment
Poor
5. Exposure baselines
Poor
6. Record-keeping
Fair

\subsubsection{Risk Control}

This element was also examined with the aid of Booklet 5 (Appendix 7). Six lines of inquiry were pursued via questions on processes for feasibility assessment and priority setting (for OHS controls), together with the effectiveness of substitution/elimination activity, procurement controls, engineering controls, administrative/work practice controls and personal protective equipment controls. Table 18 below provides a summary of the response from Facility Three to the questions raised in the booklet. Selfrating categories are as described earlier. The interviewee rated five of the six sub-elements as "Good" or "Excellent", thereby activating follow-up questions about key initiatives and success factors in these areas. 


\begin{tabular}{lcc}
\hline No. Element & $\begin{array}{c}\text { Self- } \\
\text { Rating }\end{array}$ & Key OHS Initiatives and Success \\
& Factors \\
\hline
\end{tabular}

1. Feasibility assessments Good A formal process is deployed to rank projects in terms of their importance and in terms of the feasibility of achieving a successful outcome.

2. Elimination/substitution Good There are several current examples of successful elimination and substitution effort. The hierarchy of controls is well understood and is pursued where feasible.

3. Procurement controls Fair

4. Engineering controls Good A recent modification in the Alum plant was cited as an example of a successful engineering intervention to overcome an OHS concern.

5. Work practice controls Good The facility has developed very functional guidelines for work in hot environments and work at heights. It is hoped to develop a series of similar publications.

6. PPE controls

Good The facility is seeking to upgrade eye protection in dusty environments - from safety glasses to mono-goggles.

\subsubsection{Industrial Hygiene Program Development}

This element was also examined with the aid of Booklet 5 (Appendix 7). Eleven characteristics accompany a Stage I (beginning) program, thirteen are used to describe a Stage II (improving) program and twenty are used to describe a Stage III (advanced) program. 
Based on the criteria used in Booklet 5, Facility Three has an "improving" industrial hygiene program, with some elements of an advanced program.

\subsubsection{People Initiatives}

This element was examined with the aid of Bookiet 6 (Appendix 7). Eight lines of inquiry were pursued via questions on the existence and effectiveness of behavioural safety programs, task observation programs (a component within a broader behavioural safety program), motivation and recognition programs, attitude surveys, morale surveys, safety (management) contact programs, compliance surveys relating to the use of personal protective equipment and site promotional efforts for OHS. Table 19 below provides a summary of the response from Facility Three to the questions raised in the booklet. Self-rating categories are as described earlier. The interviewee rated two of the eight sub-elements as "Good", thereby activating follow-up questions about key initiatives and

success factors attached to these. Attitude surveys have been applied, with some success. A copy of the most recent version was provided. It was noted that the site has programs under development for behavioural safety, task observations and motivation/recognition initiatives. 


\begin{tabular}{lcc} 
No. Element & $\begin{array}{c}\text { Self- } \\
\text { Rating }\end{array}$ & $\begin{array}{c}\text { Key OHS Initiatives and } \\
\text { Success Factors }\end{array}$ \\
\hline
\end{tabular}
1. Behavioural safety
2. Task observation
3. Motivation/recognition
4. Attitude surveys

\begin{abstract}
Fair
Fair
\end{abstract}
Fair
Good

5. Morale surveys

6. Management contact

7. PPE compliance

8. Promotional efforts

Poor

Poor

Fair
The facility has conducted these in the past - with useful outcomes.

Good There are wide-ranging efforts at the facility to promote EHS and several examples were cited (notice board announcements, competitions, workplace training programs, individual awareness programs ("Time-out"), publication of OHS targets and performance outcomes).

\subsubsection{Summary}

The results for Facility Three provided above may be represented as the following scores (derived from information provided by the site):

- The overall score for the compliance element was $86 \%$.

- The overall score for the organisation of work (or management enabler) element was $60 \%$. 
- The overall score for the workplace environment (or technical initiatives) element was $40 \%$.

- The overall score for the people initiatives element was $25 \%$.

Industrial Hygiene program development was characterised as "improving", with some elements of "advanced". It was assigned a score of $87.5 \%$, based on which of the program elements identified in Booklet Five appear to be in place.

21 potential benchmarking opportunities emerged from the process applied to Facility Three in this study. 13 of these relate to organisation of work (management enablers), 6 to the workplace environment (technical initiatives) and the remaining two to people initiatives.

\section{Facility Profile}

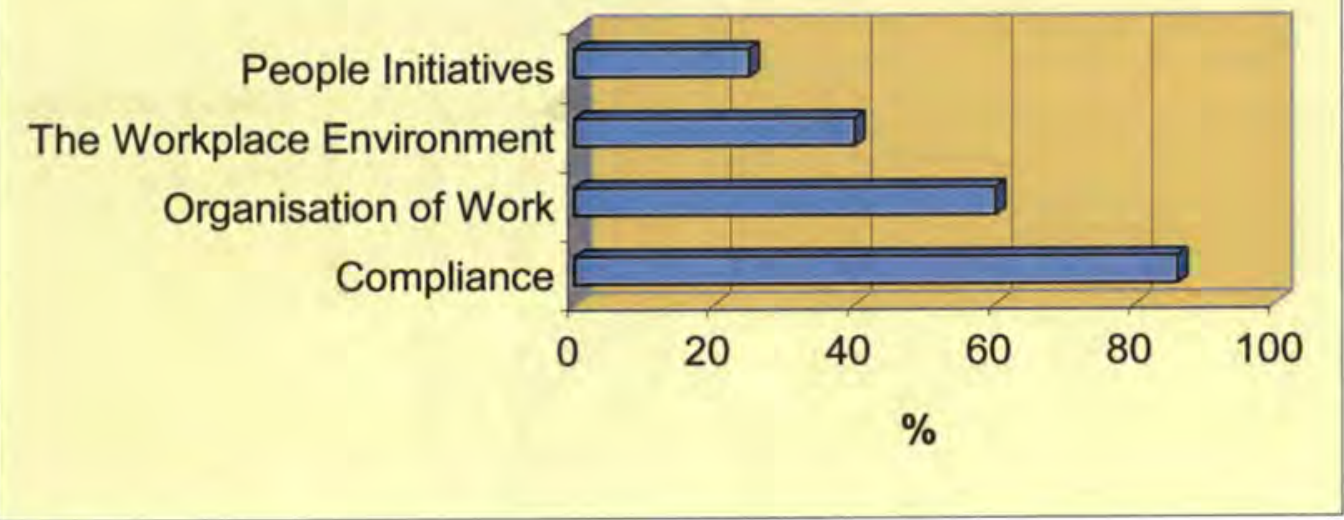

Figure 20: Facility profile - Facility Three 


\subsection{Facility Four}

\subsubsection{The Operation}

Facility Four was represented by a large contract-mining organisation. The Company operates in various parts of Australia and overseas. For the present purpose, its underground gold mining operations for a client company in the Murchison District of Western Australia is being considered. The client organisation has underground mining, above ground mining, drilling and other services carried out by separate contractors (see Facility Five description).

The main raw materials are raw earth materials, fuels, lubricants and explosives. End products are gold-bearing ore and waste rock. Other waste materials include oil, tyres, household waste, plant and equipment.

The process engaged in at Facility Four is the extraction of ore and waste rock from two underground mines. The rock is drilled and fired with an explosive charge. Material is then loaded into haul trucks and transported to allocated locations on the surface. Workshop facilities and personnel are located throughout the operation and their purpose is to carry out repairs to plant and equipment. The general plant consists of multi-boom drills, LHD (load-haul-dump) units, haul trucks, charge-up equipment, mine service equipment and light vehicles. Housing and messing accommodation is provided on-site.

A process description is provided in Figure 21.

\subsubsection{The Workforce}

The workforce numbers 90 full-time employees - split between 63 in production, 20 in maintenance and 7 in the administration and management group. Additionally, there are 20 sub-contractor employees. A rotating 12-hour shift paftern is used, with the fly-in/fly-out workforce onsite for 14 days before having 7 days off. The company has a relatively low labour turnover compared to its peers in the contract mining industry. 


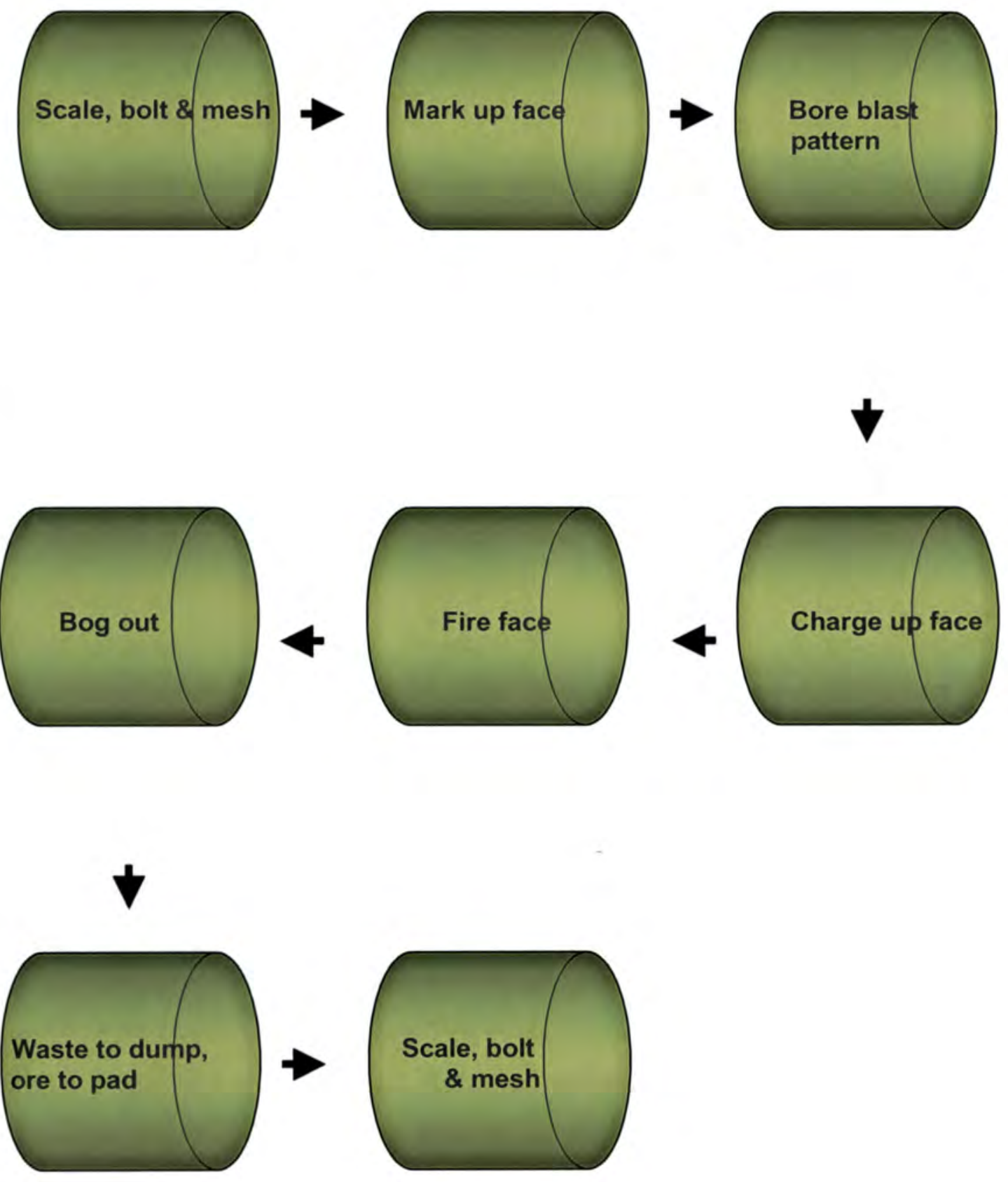

Figure 21: Schematic flowsheet - Extraction of gold-bearing ore (Facility Four)

\subsubsection{Organisation for OHS}

The Company has a safety structure built around a Group Safety Manager, an Area Safety Manager, Site Safety Manager and an OHS Training Professional. 


\subsubsection{Principal OHS Issues and Challenges}

- Physical hazards: Are those associated with the use of mobile and fixed equipment, heat, cold, fire, noise, vibration, electrical hazards, fatigue, ground movement and remote lifestyle living.

- Chemical hazards: Dust, fuels, oils and lubricants, explosives, cement products, cleaning agents, carbon monoxide and oxides of nitrogen.

- Biological hazards: Human waste, food products, personal hygiene and airborne viruses.

- Ergonomic hazards: Lifting, pushing, pulling, twisting, awkward postures, repetitive motion and similar - associated with use of office and workshop equipment, operation of mining equipment and vehicles, portable power tools and equipment repair underground.

\subsubsection{Other Relevant Information}

In 2002, Facility Four experienced a Lost Time Injury Frequency Rate of 3.2 per 1,000,000 hours worked. The dominant sources of injury are occurrences described as "struck by", "caught between" and "contact with", along with falls and over-exertion type injuries. Chemical-related injury is a minor component of the incident experience at the location.

\subsubsection{Organisational Effectiveness}

The questions posed in Booklet 2, under the heading "general OHS", are designed to provide a preliminary gauge of organisational effectiveness. The location representative responded in the affirmative to all nine questions. Thus, the facility has in place:

- A clearly expressed OHS policy.

- A philosophy and commitment to exceed minimum standards.

- Integration of OHS into the key business functions of planning, organising, leading, controlling and evaluating.

- Clearly defined OHS performance expectations and measures for all levels in the organisation.

- Injury management plans.

- Clear evidence of management involvement and commitment to OHS. 
- Clearly assigned OHS responsibilities for managers and supervisors, expressed in terms of their normal duties.

- Commitment and involvement of employees, with formal and informal structures to encourage their participation.

- A generally held view within management that Corporate investment in OHS is good business practice.

\subsubsection{Benchmarking, Best Practice and Technology Transfer}

The location representative indicated that previous efforts with respect to external OHS benchmarking have been of a very limited nature - of a type characterised by informal discussions with peers on items of common interest.

\subsubsection{Self-Ranking in OHS}

The location representative thought that his Company's operations at the site would rate in the top twenty five percent of sites within the Mining Industry of Western Australia. He based this on his knowledge of systems and procedures in place at his site, together with the incidence of injury at the facility compared to equivalent Industry sectors.

\subsubsection{Potential Benchmarking Partners}

The location representative indicated that the annual MiNEX awards, referred to earlier, are designed to identify and acknowledge elements of best practice within the Australian Mining Industry and that this would suggest a potential source of benchmarking partners.

\subsubsection{Chemical Safety Preliminary Questionnaire}

In relation to the chemical safety questionnaire presented in Part $B$ of Booklet 2, the location representative responded to all elements in the affirmative, although he noted that there were exceptions in some instances. In broad terms, the facility is characterised by the following:

- A published policy on the management of hazardous materials.

- Roles and responsibilities have been clearly assigned.

- A current hazardous materials inventory is available. 
- Material Safety Data Sheets (MSDSs) are available for every chemical entering or leaving the site.

- An MSDS will norrially accompany the first supply of a hazardous material to the location.

- There is interaction between the Purchasing and Safety/Health groups to screen new materials requested for purchase.

- There is a system to prevent non-approved chemicals coming on-site and for ensuring that those chemicals that are on-site have been approved.

- There is a system to track where and how much chemical usage occurs on-site.

- The site complies with all relevant legal requirements for the safe storage, transport, issue, use and disposal of hazardous materials employed or generated by the on-site operations.

- Labels and warning signs are posted for hazardous materials used onsite or sent elsewhere from the site.

- Sub-contractors are expected to meet similar requirements and standards with respect to the hazardous materials they bring onto the site.

\subsubsection{Flow Chart}

The purchasing arrangements at Facility Four are relatively simple. The range of chemicals used is modest and the Project Manager signs every purchase requisition.

Figure 22 represents a simplified version of the steps used at Facility Four to vet and control access of new chemicals to the site. 
Step 1

Purchase Requisition raised for a new chemical or material.

Step 2

Project Manager approves the item, subject to provision of an MSDS on first receipt of the chemical.

Step 3

Item received at the site.

Adjustments made to Chemical

Register and Chem Alert database.

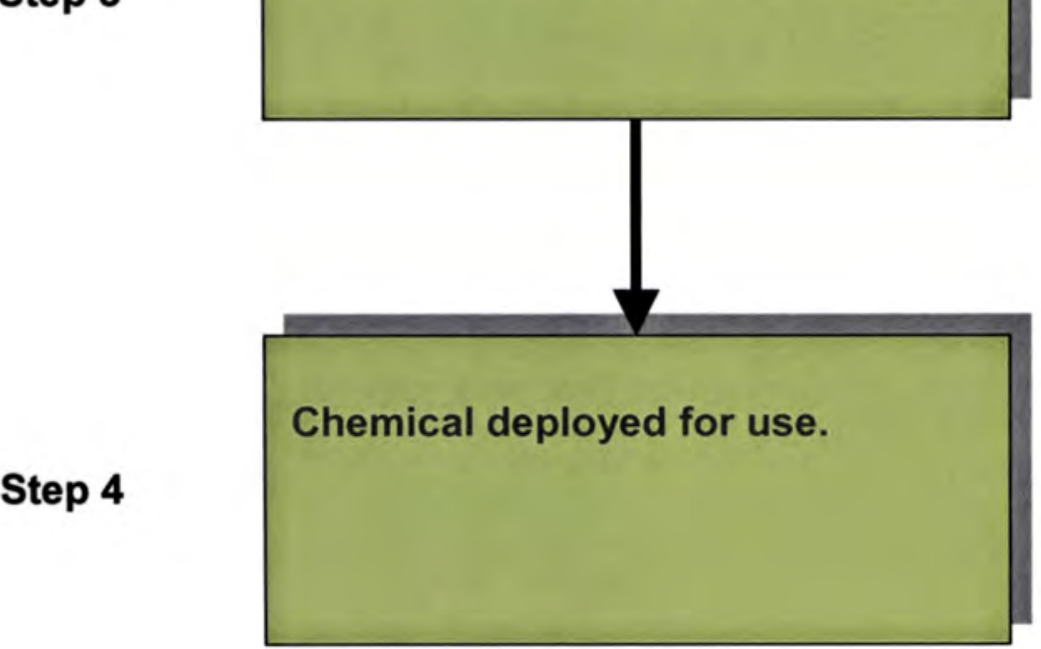

Figure 22: Acquisition of new chemicals - Facility Four 


\subsubsection{Critical Success Factors}

The site representative was asked to nominate the four most critical success factors in relation to chemical safety at the location. He nominated:

- Training modules developed for the storage, use, handling and transportation of explosives.

- Bulk supply of chemicals such as fuels and explosives (to minimise manual handling problems).

- Use of a chemical database (Chem Alert).

- Working with a minimal number of suppliers, well versed in the Company's requirements.

\subsubsection{Performance Measurement}

The traditional lagging indicators of OHS performance are quite prominent at the facility. There is a close focus on Lost Time Injury Frequency Rate (LTIFR) - defined as injury resulting in at least one complete lost shift. Medical Treatment injuries (MT/s) are tracked - these relate to injuries requiring treatment by a doctor but not resulting in a lost shift. $A$ third measure is referred to as Minor Injury (MI). This covers injuries that require first aid treatment only. In addition, severity (duration) of injuries is tracked, along with compensation costs. Incident reports, hazard logs, and equipment damage reports are other sources of information.

Leading indicators of performance have been applied in several areas of OHS. These take the form of workplace inspections, task observations, pre-start checks, workplace checklists, Job Safety Analyses (JSAs), induction and refresher training records, together with records from preshift meetings and safety meetings.

\subsubsection{Innovations in Chemical Safety}

Site operations were able to demonstrate several initiatives and innovations that could be of potential interest to other organisations. In brief, these cover:

- Portable, mobile bulk underground re-fuelling facility.

- Provision of bulk fuel, oil and grease facilities within the workshop. 
- Supply of explosives in $1000 \mathrm{~kg}$ bulk bags (to avoid use of $25 \mathrm{~kg}$ bags with attendant manual handling risks).

- Supply of non-toxic and environmentally safe degreasing products.

In a more general, OHS sense:

- Strategic planning, with very structured Key Performance Indicators (KPls).

- Deployment of the electronic B-Safe Safety Management System.

- Safety initiatives to effect cultural change and behavioural change.

- Robust structural systems that are in place to support safety efforts.

- Application of the four main daily/monthly risk management activities monthly inspection reports, daily workplace inspections, task observations and pre-start checks.

\subsubsection{Compliance}

This element was examined with the aid of Booklet 3 (Appendix 7) and the Western Australian Mines Safety and Inspection Regulations 1995.

- Reg. 7.21: The Material Safety Data Sheets (MSDSs) held on site are in the Worksafe Australia format and are current (by definition, less than 5 years old). These are sourced from an external provider, Chem Alert, and a contract is in place to give effect to this service. There is no archiving of MSDSs that relate to superseded materials or earlier formulations.

- Reg. 7.22: Containers are generally of suitable design and manufacture.

- Reg. 7.23: Many of the more substantial containers that are used on site are returned to the supplier and are then recycled.

- Reg. 7.24: Materials coming onto the site appear to be labelled in accordance with the Australian Dangerous Goods Code. However, there appears to be some variability in practices attached to the use of labels when transferring chemicals from supplier-provided containers to smaller, site-issued containers.

- Reg. 7.25: MSDS registers exist and information is available to employees. However, some deficiencies exist in relation to details of 
risk assessments carried out under Regulation 7.27 and mechanisms for updating MSDSs in the register.

- Reg. 7.26: Some deficiencies exist in relation to work procedures, rules and training that are applied to ensure that persons working in enclosed spaces are aware of the potential risks attached to the use of hazardous substances in those spaces.

- Reg. 7.27: Some deficiencies exist in relation to the conduct of formal risk assessments and formal documentation to go with this.

- Reg. 7.28: The hierarchy of controls is understood and deployed on the site to a limited extent. In general, suitable procedures and arrangements apply to the application of engineering, administrative and work practice controls, and personal protective equipment controls.

- Reg. 7.29: Atmospheric monitoring programs are undertaken, with emphasis on blast fume and respirable dust levels. Most air contaminant levels are below those identified as acceptable in Worksafe Australia's Exposure Standards.

- Reg. 7.30: Health surveillance programs are limited to those specified in the Government's Mines Medical requirements.

- Reg. 4.24-4.29: The site appears to meet regulatory requirements relating to provision of appropriate equipment, facilities and services for first aid.

- Reg. 4.13: The site appears to meet most regulatory requirements for training of employees and the maintenance of training records. Some opportunities exist in relation to training employees in the use of an MSDS.

\subsubsection{Organisation of Work}

This element was examined with the aid of Booklet 4 (Appendix 7). Twenty-two lines of inquiry were pursued under the principal headings of leadership, commitment, planning, organisation, training, communication and measurement. Table 20 below provides a summary of the response from Facility Four to the questions raised in the booklet. Self-ratings fall into four categories - Poor, Fair, Good or Excellent. The interviewee rated 
fifteen of the twenty-two elements as "Good", thereby activating follow-up questions about key initiatives and success factors. Elements allocated a "Fair" rating in the table that follow are usually associated with programs or efforts that are still at an early stage of development or are carried out sporadically. "Poor" or "Not used" was applied as a rating in the sense that the element was non-existent at the location. There may be valid reasons for that. It may not have been needed, it may not be appropriate, or an alternative approach may have been used to achieve the same end point. 


\section{No. Element Self- Key OHS Initiatives and Success Factors Rating}

1. Management visibility

2. Policy enunciation

3. Tracking performance

4. Financial resources

5. Internal resources

6. External resources

7. Employee involvement

8. Strategic planning

9. Target-setting
Good There is good leadership visibility within the mine and effective communications at pre-shift meetings.

Fair

Good Performance to client requirements is closely monitored. This is an important factor in attracting further business

Exc. Budgets carry provision for local OHS initiatives eg. training, equipment, materials).

Exc. Supervisors conduct safety meetings, pre-shift meetings (safety a component) and complete Job Hazard Analyses (JHAs) on hazardous tasks.

Good There is ready access to the resources of the Department of Industry and Resources, including Workmen's Inspectors of Mines, support from product suppliers and from consultants performing CONTAM sampling and similar.

Poor

Good The Company conducts an annual crosssite safety conference for this purpose.

Good Industry performance trends are tracked, plans are formulated and there is budgetary support for this. 
Table 20

Organisation of Work - Facility Four (Continued)

$\begin{array}{ccc}\text { No. Element } & \begin{array}{c}\text { Self- } \\ \text { Rating }\end{array} & \text { Key OHS Initiatives and Success } \\ & \text { Factors }\end{array}$

10. Contractor plans

Fair

11. Organisational structure

Fair

12. OHS integration

Good OHS is embedded in the overall management system. The Managing

Director briefs sites on safety performance and client safety expectations are quite high.

13. Departmental integration

Fair

14. Induction training

Good This comes in three parts. There is a MARCSTA general induction, followed by a site induction and then a jobspecific induction.

15. Refresher training

Good A strong task observation program is used as follow-up training tool. Postincident investigations may lead to certain forms of refresher training.

16. Safety rep training

Good Training is built around statutory requirements (introductory training), together with opportunities attached to incident investigation and workplace inspections.

17. Effectiveness of training

Good There is a practical assessment by training personnel, together with supervisor feedback.

18. Hazard communication

Good Hazards identified are raised at preshift meetings. Communications also occur via safety notice boards. 


No. Element $\quad \begin{gathered}\text { Self- Key OHS Initiatives and Success Factors } \\ \text { Rating }\end{gathered}$
Ret

19. Communication of Poor targets

20. Delivery of feedback Poor

21. Use of lagging

Good Several measures are applied eg. Lost Time indicators Injuries, Medical Treatment Injuries, Minor Injuries, incident reports, duration days, compensation costs and equipment damage costs.

22. Use of leading

Good These take the form of workplace inspection indicators outcomes, task observations, pre-start checks, workplace checklists, JSAs, induction and training records, pre-shift meetings and safety meetings.

\subsubsection{Hazard Recognition}

This element was examined with the aid of Booklet 5 (Appendix 7). Three lines of inquiry were pursued via questions on walk-through surveys (an established technique for identifying hazards, evaluating work practices and checking the efficacy of existing controls), job dictionaries (that itemise jobs and tasks, as a preliminary to assignment of exposure information and the assessment of risk) and chemical inventories. Table 21 below provides a summary of the response from Facility Four to the questions raised in the booklet. Self-rating categories are as described earlier. The interviewee rated one of the sub-elements as "Good", thereby activating follow-up questions about key initiatives and success factors for that element. 


\begin{tabular}{ccc}
\hline No. & Self- & Key OHS Initiatives and Success \\
Nont & Rating & Factors \\
\hline
\end{tabular}

1. Walk-through surveys Good Inspection activities are built around workplace checks at each new worksite, monthly workplace inspections and six-monthly BSAFE audits.

2. Job dictionaries Not

used

3. Chemical inventories Poor

\subsubsection{Risk Assessment}

This element was also examined with the aid of Booklet 5 (Appendix 7). Six lines of inquiry were pursued via questions on the existence and effectiveness of sampling plans and schedules, qualitative assessment programs, quantitative assessment programs, statistical treatment of sampling results, exposure baselines and record-keeping. Table 22 below provides a summary of the response from Facility Four to the questions raised in the booklet. Self-rating categories are as described earlier. The interviewee rated one of the sub-elements as "Good", thereby activating follow-up questions about key initiatives and success factors for that element. 
Table 22

Risk Assessment - Facility Four

\begin{tabular}{ccc} 
No. Element & $\begin{array}{c}\text { Self- } \\
\text { Rating }\end{array}$ & Key OHS Initiatives and Success \\
& Factors \\
\hline
\end{tabular}

1. Sampling plans

Good Quarterly sampling is carried out according to the Government's CONTAM requirements.

2. Qualitative assessments

3. Quantitative assessments

.4. Statistical treatment

5. Exposure baselines

6 Record-keeping
Poor

Fair

Fair

Fair

Fair

\subsubsection{Risk Control}

This element was also examined with the aid of Booklet 5 (Appendix 7). Six lines of inquiry were pursued via questions on processes for feasibility assessment and priority setting (for OHS controls), together with the effectiveness of substitution/elimination activity, procurement controls, engineering controls, administrative/work practice controls and personal protective equipment controls. Table 23 below provides a summary of the response from Facility Four to the questions raised in the booklet. Selfrating categories are as described earlier. The interviewee rated three of the sub-elements as "Good", thereby activating follow-up questions about key initiatives and success factors for those elements. 


\begin{tabular}{cc} 
Nelf- & $\begin{array}{c}\text { Sel } \\
\text { Rating } \quad \text { Key OHS Initiatives and Success } \\
\text { Factors }\end{array}$ \\
\hline
\end{tabular}

1. Feasibility assessments Poor

2. Elimination/substitution Poor

3. Procurement controls Poor

4. Engineering controls Good The suppliers have a role in this activity and examples are available of changes they have introduced.

5. Work practice controls Good Competency training is applied where chemicals are part of the task.

Chemical use is catered for in Job Safety Analyses.

6. PPE controls

Good These are supported by supervisory practices, together with training and awareness packages. There is ready availability and deployment of PPE conforming to Australian Standards, and meeting comfort and practicability criteria.

\subsubsection{Industrial Hygiene Program Development} This element was also examined with the aid of Booklet 5 (Appendix 7). Eleven characteristics accompany a Stage I (beginning) program, thirteen are used to describe a Stage II (improving) program and twenty are used to describe a Stage III (advanced) program.

Based on the criteria used in Booklet 5, Facility Four has a "beginning" industrial hygiene program. 


\subsubsection{People Initiatives}

This element was examined with the aid of Booklet 6 (Appendix 7). Eight lines of inquiry were pursued via questions on the existence and effectiveness of behavioural safety programs, task observation programs (a component within a broader behavioural safety program), motivation and recognition programs, attitude surveys, morale surveys, safety (management) contact programs, compliance surveys relating to the use of personal protective equipment and site promotional efforts for OHS. Table 24 below provides a summary of the response from Facility Four to the questions raised in the booklet. Self-rating categories are as described earlier. The interviewee rated one of the eight sub-elements as "Good", thereby activating follow-up questions about key initiatives and success factors attached to that element.

\section{Table 24}

People Initiatives - Facility Four

\begin{tabular}{ccc} 
No. Element & $\begin{array}{c}\text { Self- } \\
\text { Rating }\end{array}$ & Key OHS Initiatives and Success \\
& Factors \\
\hline
\end{tabular}

1. Behavioural safety

2. Task observation

3. Motivation/recognition

4. Attitude surveys

5. Morale surveys

6. Management contact

7. PPE compliance

8. Promotional efforts
Not used

Good Supervisor-led task observations are supported by comprehensive guidance material.

Fair

Not used

Fair

Not used

Poor

Poor 


\subsubsection{Summary}

The results for Facility Four provided above may be represented as the following scores:

- The overall score for the compliance element was $73 \%$.

- The overall score for the organisation of work (or management enabler) element was $68 \%$.

- The overall score for the workplace environment (or technical initiatives) element was $33 \%$.

- The overall score for the people initiatives element was $13 \%$.

Industrial Hygiene program development was characterised as "beginning" and assigned a score of $51 \%$ - based on the descriptors provided in Booklet 5.

Twenty-one potential benchmarking opportunities emerged from the process applied to Facility Four in this study. Fifteen of these relate to organisation of work (management enablers), five to the workplace environment (technical initiatives) and the remaining one to people initiatives.

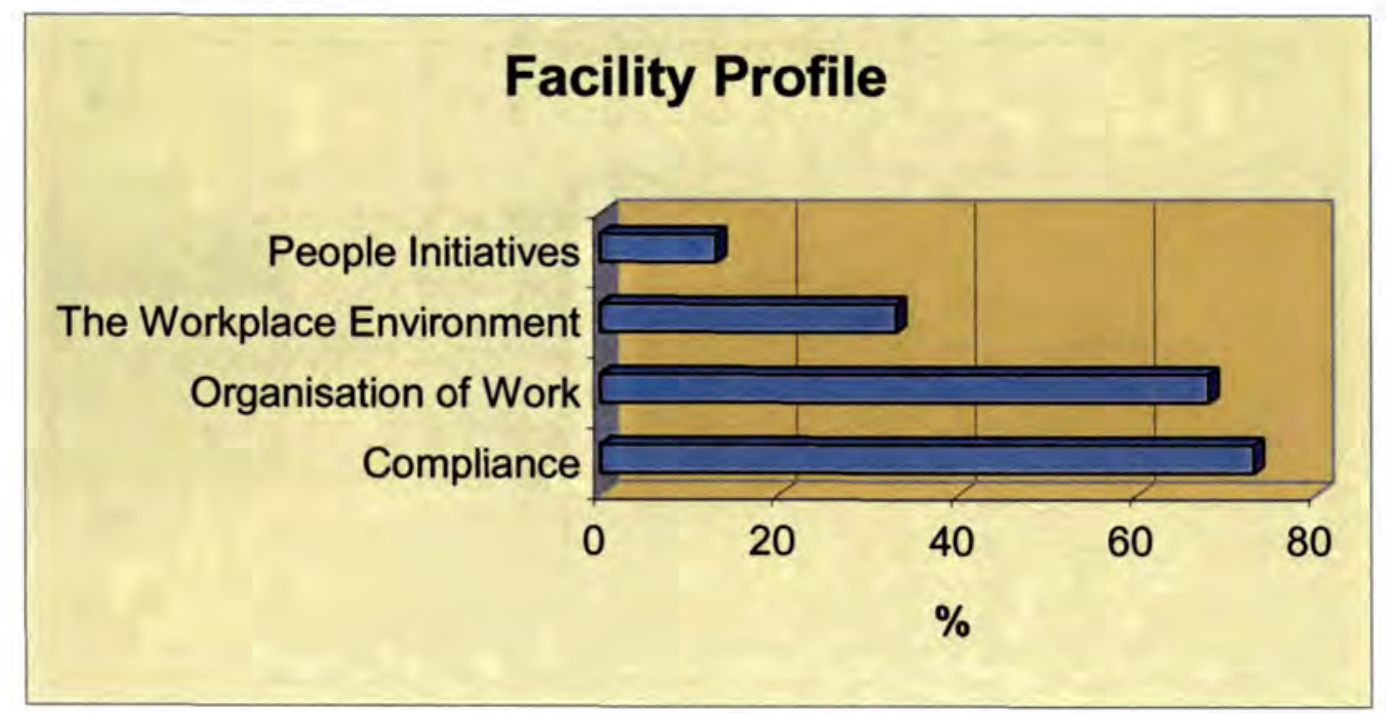

Figure 23: Facility profile - Facility Four 


\subsection{Facility 5}

\subsubsection{The Operation}

Facility Five was a gold mining, milling and extraction operation in the Murchison District of Western Australia, some $560 \mathrm{~km}$ north-east of Perth. Mining of ore is carried out by mining contractors and encompasses above- and below-ground operations. The Company carries out gold extraction via a Carbon-in-Leach (CIL) Process Plant. It generates its own power. The main raw materials for the facility are sodium cyanide, caustic soda, hydrochloric acid, lime, LPG, liquid oxygen and diesel fuel. The end product is gold bullion.

Material left over after the extraction of gold is disposed of to a tailings area. There is some re-use of old tailings deposits and about $25 \%$ of the materials processed by the mill are from this source. The gold content is high enough to make extraction from waste an economically feasible exercise.

A simplified description of the CIL Process is provided in Figure 24. 


\section{Ore from Mine}

\section{\& Tailings Deposit}

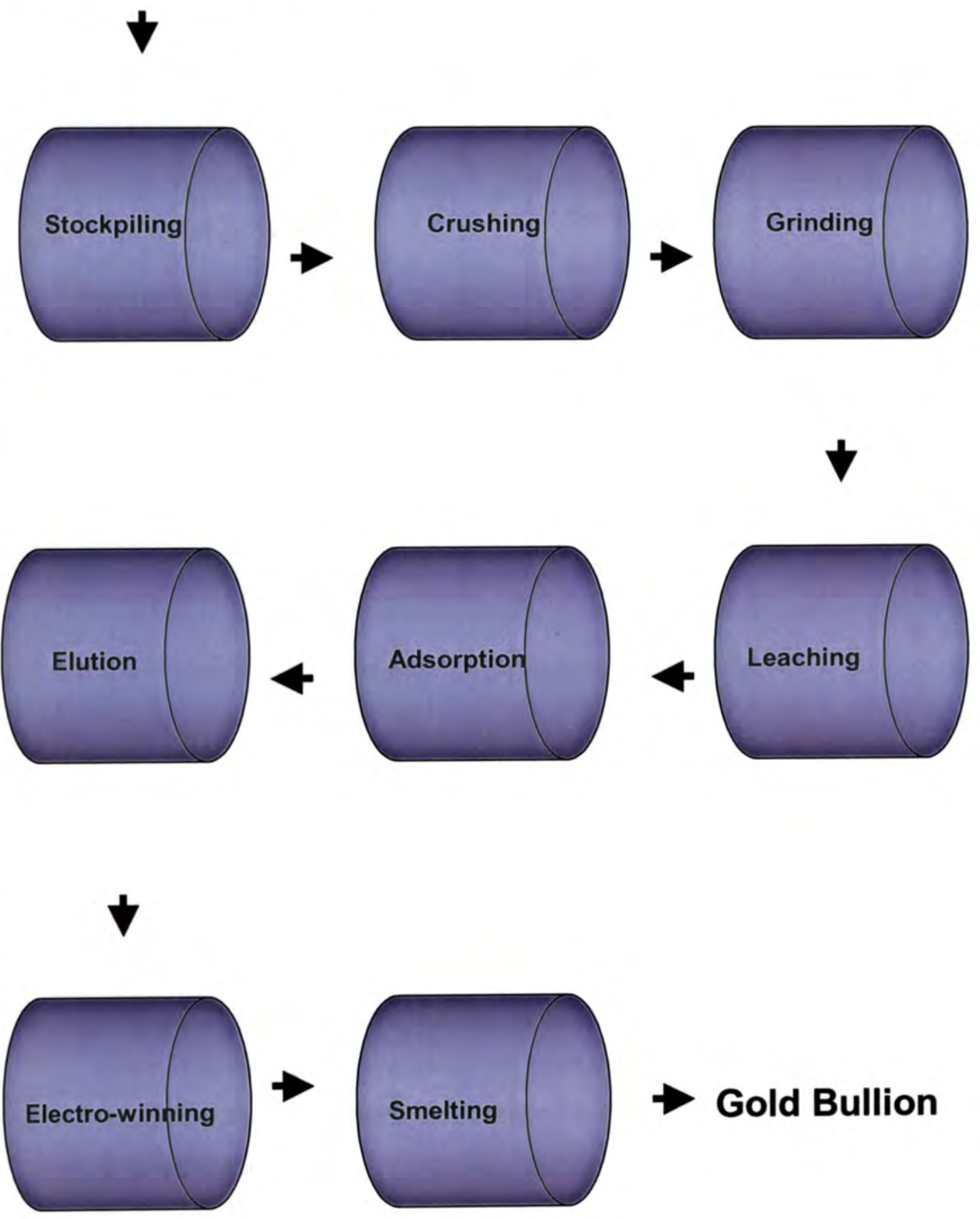

Figure 24: Schematic flowsheet - ore to gold bullion (Facility Five) 


\subsubsection{The Workforce}

There are 83 full-time, permanent Company employees at the site - 13 in administration, 22 in mining, 31 in metallurgy, 10 in geology and 7 in exploration. There are three principal contracting organisations on site and these are engaged in underground mining, surface mining and diamond drilling operations. In total, there are 304 people at the site. The mine is run on a continuous shift basis. Arrangements are variable, although most employees work a nine-day fortnight or a two-weeks on, one week off pattern.

\subsubsection{Organisation for $\mathrm{OHS}$}

The location has a loss control coordinator and a loss control administrator. The coordinator reports functionally to a loss control manager who has broader corporate responsibilities. There is an emphasis on first-aid training and emergency response capability at the site.

\subsubsection{Principal OHS Issues and Challenges}

- Physical hazards: Noise, vibration, ionising radiation, and thermal stress.

- Chemical hazards: Gold-bearing ore dust, tallings dust, alkaline materials, acid materials, heavy metals, welding fume, combustion gases and industrial chemicals (garnet-based abrasive blasting materials, LPG, oxygen and other compressed gases, cleaning agents, oils and greases, surfactants, adhesives, sealants, solvents, spray paint, insecticides, herbicides and similar).

- Biological hazards: None apparent.

- Ergonomic hazards: Lifting, pushing, puiling, twisting, awkward postures, repetitive motion and similar have potential to produce ergonomic injury.

\subsubsection{Other Relevant Information}

The facility changed hands last year and now belongs to an international mining operation. It benefits from the OHS knowledge and experience within the wider organisation. The site also draws from the procedures 
and systems that were available to it under previous ownership arrangements.

The Department of Minerals and Petroleum Resources of Western Australia conducted an audit of management systems at Facility Five in December 2001. The site obtained a score of $94 \%$ - based on the structure described by DMPR (2002) and referred to earlier. Figures provided by the State Mining Engineer (M. Knee, personal communication, 2003) indicate that 128 mine sites and mineral processing operations in Western Australia have been audited in this way over the past five years. Scores ranged from $35 \%$ to $99 \%$, with a mean of $76 \%$. Thus, the site is in the top bracket of performers, as measured by the Department's audit system.

In 2002, the site experienced a Lost Time injury Frequency Rate of 2.4 per $1,000,000$ hours for all employees (including contractors) and a zero rate for Company employees only.

\subsubsection{Organisational Effectiveness}

The questions posed in Booklet 2, under the heading "general $\mathrm{OHS}^{\text {", are }}$ designed to provide a preliminary gauge of organisational effectiveness. The location representative responded in the affirmative to all nine questions. Thus, the facility has in place:

- A clearly expressed OHS policy.

- A philosophy and commitment to exceed minimum standards.

- Integration of OHS into the key business functions of planning, organising, leading, controlling and evaluating.

- Clearly defined OHS performance expectations and measures for all levels in the organisation.

- Injury management plans.

- Clear evidence of management involvement and commitment to OHS.

- Clearly assigned OHS responsibilities for managers and supervisors, expressed in terms of their normal duties. 
- Cormmitment and involvement of employees, with formal and informal structures to encourage their participation.

- A generally held view within management that Corporate investment in OHS is good business practice.

\subsubsection{Benchmarking, Best.Practice and Technology Transfer}

The location representative indicated that previous efforts with respect to external OHS benchmarking have been of a very limited nature - of a type characterised by site visits and unstructured discussions on a few items of interest.

\subsubsection{Self-Ranking in OHS}

The location representative thought that her site would rate in the top ten percent of sites within the Mining Industry of Westem Australia. She based this on her knowledge of systems and procedures in place at her site, together with the low incidence of chemical-related injury and nearmiss experience at the facility. She also had some anecdotal information from her external professional network and from suppliers and others who move from site to site.

\subsubsection{Potential Benchmarking Partners}

The location representative nominated two organisations in South Africa as most admired in terms of performance and reputation for excellence in OHS. These were South African Breweries and the South African Paper and Pulp Industry (SAPPI). She acknowledged that the Western Mining Corporation, a previous owner of the facility, had contributed a lot to the OHS knowledge and systems within her sile.

\subsubsection{Chemical Safety Preliminary Questionnaire}

In relation to the chemical safety questionnaire presented in Part $B$ of Booklet 2, the location representative responded to all elements in the affirmative. Thus, the facility is characterised by the following:

- A published policy on the management of hazardous materials.

- Roles and responsibilities have been clearly assigned. 
- A current hazardous materials inventory is available.

- Material Safety Data Sheets (MSDSs) are available for every chemical entering or leaving the site.

- An MSDS will normally accompany the first supply of a hazardous material to the location.

- There is interaction between the Purchasing and Safety/Health groups to screen new materials requested for purchase.

- There is a system to prevent non-approved chemicals coming on-site and for ensuring that those chemicals that are on-site have been approved.

- There is a system to track where and how much chemical usage occurs on-site.

- The site complies with all relevant legal requirements for the safe storage, transport, issue, use and disposal of hazardous materials employed or generated by the on-site operations.

- Labels and warning signs are posted for every hazardous material used on-site or sent elsewhere from the site.

- Contractors are expected to meet similar requirements and standards with respect to the hazardous materials they bring onto the site.

\subsubsection{Flow Chart}

The purchasing arrangements at Facility Five are relatively simple. The range of chemicals used is modest and the Loss Control Coordinator signs every purchase requisition that relates to hazardous materials.

Figure 25 represents a simplified version of the steps used at Facility Five to vet and control access of new chemicals to the site. 


\section{Step 1}

Purchase Requisition raised for a new chemical

Step 2

The Site Loss Control Coordinator approves the item, subject to provision of an MSDS on first receipt of the chemical.

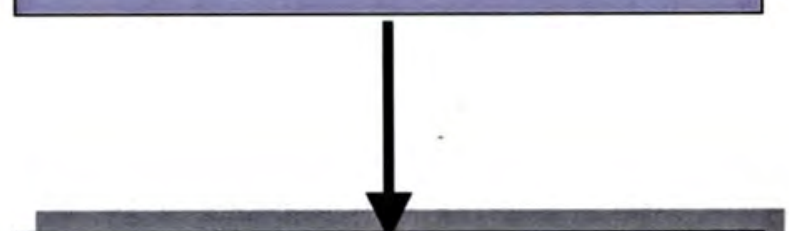

Item received at the site. Adjustments made to the Chemical Register and Chem Alert database.

Step 3

Step 4

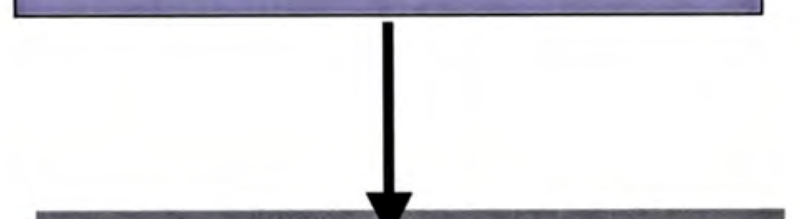

Chemical deployed for use.

Figure 25: Acquisition of new chemicals - Facility Five 


\subsubsection{Critical Success Factors}

The site representative was asked to nominate the four most critical success factors in relation to chemical safety at the location. She nominated:

- Use of the Chem Alert materials information service.

- Adherence to procedures.

- Use of a system to address ordering, vetting, supply and distribution of hazardous materials.

- Deployment of personal protective equipment systems and procedures.

\subsubsection{Performance Measurement}

The traditional lagging indicators of OHS performance are quite prominent at the facility. There is a close focus on Lost Time Injury Frequency Rate (LTIFR) - defined as injury resulting in at least one complete lost shift. Medical Treatment Injuries (MTis) are tracked - these relate to injuries requiring treatment by a doctor but not resulting in a lost shift. A third measure is referred to as Minor Injury (Ml). This covers injuries that require first aid treatment only.

Leading indicators of performance take the form of workplace inspection outcomes, task observations, pre-start checks, workplace checklists, job safety analyses, induction and on-going training records, pre-shift meetings and safety meetings.

\subsubsection{Innovations in Chemical Safety}

The location was able to demonstrate several strengths that could be of potential interest to other organisations. Most relate to aspects of OHS that are broader than chemical safety.

Details are provided in the sections that follow but, in brief, these cover:

- The Safety Management System

- The "Whole of Mining Risk Assessment".

- Reporting protocols.

- Site induction training programs based on use of CD-Rom tools. 
- Accident investigation.

\subsubsection{Compliance}

This element was examined with the aid of Booklet 3 (Appendix 7) and the Western Australian Mines Safety and Inspection Regulations 1995.

- Reg. 7.21: The Material Safety Data Sheets (MSDSs) held on site are in the Worksafe Australia format and are current (by definition, less than 5 years old). In the main, an extemal party that specialises in this area provides these. Hard copy versions are kept in crisis management lockers.

- Reg. 7.22: Containers are generally of suitable design and manufacture. There was some variability around the site in terms of the practices suirounding the transfer of bulk supplies to more suitable smaller containers.

- Reg. 7.23: Many of the more substantial containers that are used on site are returned to the supplier and are then recycled. Those that remain are disposed of to a local landfill site.

- Reg. 7.24: Materials coming onto the site appear to be labelled in accordance with the Australian Dangerous Goods Code. However, there appears to be some variability in practices attached to the use of labels when transferring chemicals from supplier-provided containers to smaller, site-issued containers.

- Reg. 7.25: MSDS information is freely accessible to employees and the MSDS register contains details of risk assessments carried out in accordance with the regulations.

- Reg. 7.26: Work procedures, rules and training are applied to ensure that persons working in enclosed spaces are aware of the potential risks attached to the use of hazardous substances in those spaces.

- Reg. 7.27: Qualitative and quantitative risk assessments have been carried out, as appropriate, and are formally documented.

- Reg. 7.28: The hierarchy of controls is understood and deployed on the site. Suitable procedures and arrangements apply to the 
application of engineering, administrative and work practice controls, and personal protective equipment controls.

- Reg. 7.29: Atmospheric monitoring programs are carried out by an external organisation, "Baseline". Most, but not all, exposure levels are consistently below those identified in Worksafe Australia's Exposure Standards.

- Reg. 7.30: Health surveiliance programs are also highly developed. These are under the direction of a part-time Occupational Physician. Health assessments are carried out at initial employment and periodically thereafter.

- Reg. 4.24-4.29: The site appears to meet regulatory requirements relating to provision of appropriate equipment, facilities and services for first aid.

- Reg. 4.13: The site appears to meet all regulatory requirements for training of employees and the maintenance of training records.

\subsubsection{Organisation of Work}

This element was examined with the aid of Booklet 4 (Appendix 7). Twenty-two lines of inquiry were pursued under the principal headings of leadership, commitment, planning, organisation, training, communication and measurement. Table 25 below provides a summary of the response from Facility Five to the questions raised in the booklet. Self-ratings fall into four categories - Poor, Fair, Good or Excellent. The interviewee rated 20 of the 22 elements as "Good" or "Excellent", thereby activating followup questions about key initiatives and success factors. Elements allocated a "Fair" rating in the table that follow are usually associated with programs or efforis that are still at an early stage of development or are carried out sporadically. 
Table 25

Organisation of Work - Facility Five

No. Element Key OHS Initiatives and Success Factors

1. Management visibility

2. Policy enunciation

3. Tracking performance

4. Financial resources

5. Internal resources

6. External resources

7. Employee involvement

8. Strategic planning

9. Target-setting

10. Contractor plans
Good Senior managers lead by example and are prominent at safety meetings.

Good Wall-mounted policy statements found in many well-accessed plant locations.

Policy statements feature in many of the location's publications.

Good Key performance indicators for safety are tracked at management meetings and safety meetings.

Good Departmental budgets carry provision for local OHS initiatives (eg. training, equipment, engineering and capital works).

Good The site appears to be adequately resourced with safety, health and emergency services personnel.

Good The facility contracts specialist groups to perform tasks such as chemical audits and air monitoring.

Good There is a visible employee involvement in departmental and crew level safety activities.

Good A high level of attention is applied to strategic planning for OHS.

Good Targets are set on both short term and annual bases.

Fair 


No. Element Self- Key OHS Initiatives and Success Factors
Rating

11. Organisational structure Good The organisational structure encourages interaction of OHS professionals with line management and shop floor personnel.

12. OHS integration

13. Departmental integration

14. Induction training

15. Refresher training

16. Safety rep training

17. Effectiveness of training

18. Hazard communication

19. Communication of targets
Good Site and departmental OHS plans are integrated with broader Corporate plans.

Good Departmental OHS efforts are developed from broader site plans and commitments.

Exc. Induction is structured around a one-day MARCSTA program (generic), followed by a local/departmental program.

Exc. Follow-up training is delivered via safety meetings, toolbox sessions and similar.

Good The introductory course is followed by further skills training and awarenessbuilding.

Fair

Good Many approaches are used in this area. These include accident/incident report forms, hazard logs, safety alerts and notice board items, the Chem Alert database, awareness training and similar.

Good Expectations and targets are communicated via the line management system and a variety of hard copy and electronic systems. 


No. Element $\begin{gathered}\text { Self- Key OHS Initiatives and Success Factors } \\ \text { Rating }\end{gathered}$

20. Delivery of feedback Good There are many channels for feedback - from "toolbox" meetings at the shop floor level through to electronic and hard copy reports, newsletters and other presentations.

21. Use of lagging

Good The system is centred on the reporting of lost indicators time injuries, medical treatment injuries and minor injuries. This is well deployed and understood.

22. Use of leading indicators

Good These take the form of workplace inspection outcomes, task observations, pre-start checks, workplace checklists, job safety analyses, induction and training records, preshift meetings and safety meetings.

\subsubsection{Hazard Recognition}

This element was examined with the aid of Booklet 5 (Appendix 7). Three lines of inquiry were pursued via questions on walk-through surveys (an established technique for identifying hazards, evaluating work practices and checking the efficacy of existing controls), job dictionaries (that itemise jobs and tasks, as a preliminary to assignment of exposure information and the assessment of risk) and chemical inventories. Table 26 below provides a summary of the response from Facility Five to the questions raised in the booklet. Self-rating categories are as described earlier. The interviewee rated all three of the sub-elements as "Good", thereby activating follow-up questions about key initiatives and success factors. 


\begin{tabular}{lll}
\hline No. Element & $\begin{array}{c}\text { Self- } \\
\text { Rating }\end{array}$ & $\begin{array}{c}\text { Key OHS Initiatives and Success } \\
\text { Factors }\end{array}$ \\
\hline 1. Walk-through surveys & Good & $\begin{array}{l}\text { The Loss Control Coordinator } \\
\text { conducts these. Departmental } \\
\text { inventories are checked against } \\
\text { chemicals found. }\end{array}$ \\
2. Job dictionaries & Good & $\begin{array}{l}\text { Each job is defined in relation to } \\
\text { potential exposures. } \\
\text { Departmental inventories are in place } \\
\text { and used in training, awareness- } \\
\text { building and auditing applications. }\end{array}$ \\
3. Chemical inventories & Good
\end{tabular}

\subsubsection{Risk Assessment}

This element was also examined with the aid of Booklet 5 (Appendix 7). Six lines of inquiry were pursued via questions on the existence and effectiveness of sampling plans and schedules, qualitative assessment programs, quantitative assessment programs, statistical treatment of sampling results, exposure baselines and record-keeping. Table 27 below provides a summary of the response from Facility Five to the questions raised in the booklet. Self-rating categories are as described earlier. The interviewee rated five of the six sub-elements as "Good" or "Excellent", thereby activating follow-up questions about key initiatives and success factors. 
Table 27

Risk Assessment - Facility Five

\begin{tabular}{lcc}
\hline No. Element & $\begin{array}{c}\text { Self- } \\
\text { Rating }\end{array}$ & Key OHS Initiatives and Success \\
& Factors \\
\hline
\end{tabular}

1. Sampling plans

2. Qualitative assessments

3. Quantitative assessments

\section{Statistical treatment \\ 5. Exposure baselines}

6 Record-keeping
Good These are established on an annual basis. Essentially, these respond to CONTAM requirements.

Good The Loss Control Coordinator carries out these assessments on a regular basis.

Good Personal exposure measurements are conducted with appropriate members of the workforce.

Fair

Good Baselines have been established for all significant exposures at the site.

Exc. This is built around hard copy and electronic versions of key records.

\subsubsection{Risk Control}

This element was also examined with the aid of Booklet 5 (Appendix 7). Six lines of inquiry were pursued via questions on processes for feasibility assessment and priority setting (for OHS controls), together with the effectiveness of substitution/elimination activity, procurement controls, engineering controls, administrative/work practice controls and personal protective equipment controls. Table 28 below provides a summary of the response from Facility Five to the questions raised in the booklet. Selfrating categories are as described earlier. The interviewee rated all six of the sub-elements as "Good", thereby activating follow-up questions about key initiatives and success factors. 
Table 28

Risk Control - Facility Five

Self-

No. Element Rating Key OHS Initiatives and Success Factors

1. Feasibility assessments Good A formal process is deployed to rank projects in terms of their importance and in terms of the feasibility of achieving a successful outcome.

2. Elimination/substitution Good There are several current examples of successful elimination and substitution effort.

The hierarchy of controls is well understood and is pursued where feasible.

3. Procurement controls Good The Loss Control Coordinator interacts with Purchasing and Stores functions when dealing with new chemicals proposed for use on the site.

4. Engineering controls

Good Examples were given of several successful engineering projects that sought to address OHS concerns at the site.

5. Work practice controls Good Procedures and administrative arrangements are supported by supervisory practices, together with training and awareness packages.

6. PPE controls

Good There is ready availability and deployment of PPE conforming to Australian Standards, and meeting comfort and practicability criteria. 


\subsubsection{Industrial Hygiene Program Development}

This element was also examined with the aid of Booklet 5 (Appendix 7). Eleven characteristics accompany a Stage I (beginning) program, thirteen are used to describe a Stage II (improving) program and twenty are used to describe a Stage III (advanced) program.

Based on the criteria used in Booklet 5, Facility Five has an "improving" industrial hygiene program, with some elements of "advanced".

\subsubsection{People initiatives}

This element was examined with the aid of Booklet 6 (Appendix 7). Eight lines of inquiry were pursued via questions on the existence and effectiveness of behavioural safety programs, task observation programs (a component within a broader behavioural safety program), motivation and recognition programs, attitude surveys, morale surveys, formal safety (management) contact programs, compliance surveys relating to the use of personal protective equipment and site promotional efforts for OHS. Table 29 below provides a summary of the response from Facility Five to the questions raised in the booklet. Self-rating categories are as described earlier. The interviewee rated one of the eight sub-elements as "Good", thereby activating follow-up questions about associated initiatives and success factors. 
Table 29

People Initiatives - Facility Five

$\begin{array}{ccc}\text { No. Element } & \begin{array}{c}\text { Self- } \\ \text { Rating }\end{array} & \text { Key OHS Initiatives and Success } \\ & \text { Factors }\end{array}$
1. Behavioural safety
Not used
2. Task observation
Not used
3. Motivation/recognition
Fair
4. Attitude surveys
Not used
5. Morale surveys
Not used
6. Formal management
Fair contact programs

7. PPE compliance

Not used

8. Promotional efforts

Good Signage and notice board initiatives are prominent.

\subsubsection{Summary}

The results for Facility Five provided above may be represented as the following scores:

- The overall score for the compliance element was $95 \%$.

- The overall score for the organisation of work (or management enabler) element was $91 \%$.

- The overall score for the workplace environment (or technical initiatives) element was $93 \%$.

- The overall score for the people initiatives element was $13 \%$.

Industrial Hygiene program development was characterised as "improving", with some elements of "advanced". It was assigned a score of $79 \%$, based on which of the program elements identified in Booklet 5 appear to be in place. 
35 potential benchmarking opportunities emerged from the process applied to Facility Five in this study. 20 of these relate to organisation of work (management enablers), 14 to the workplace environment (technical initiatives) and the remaining one to people initiatives.

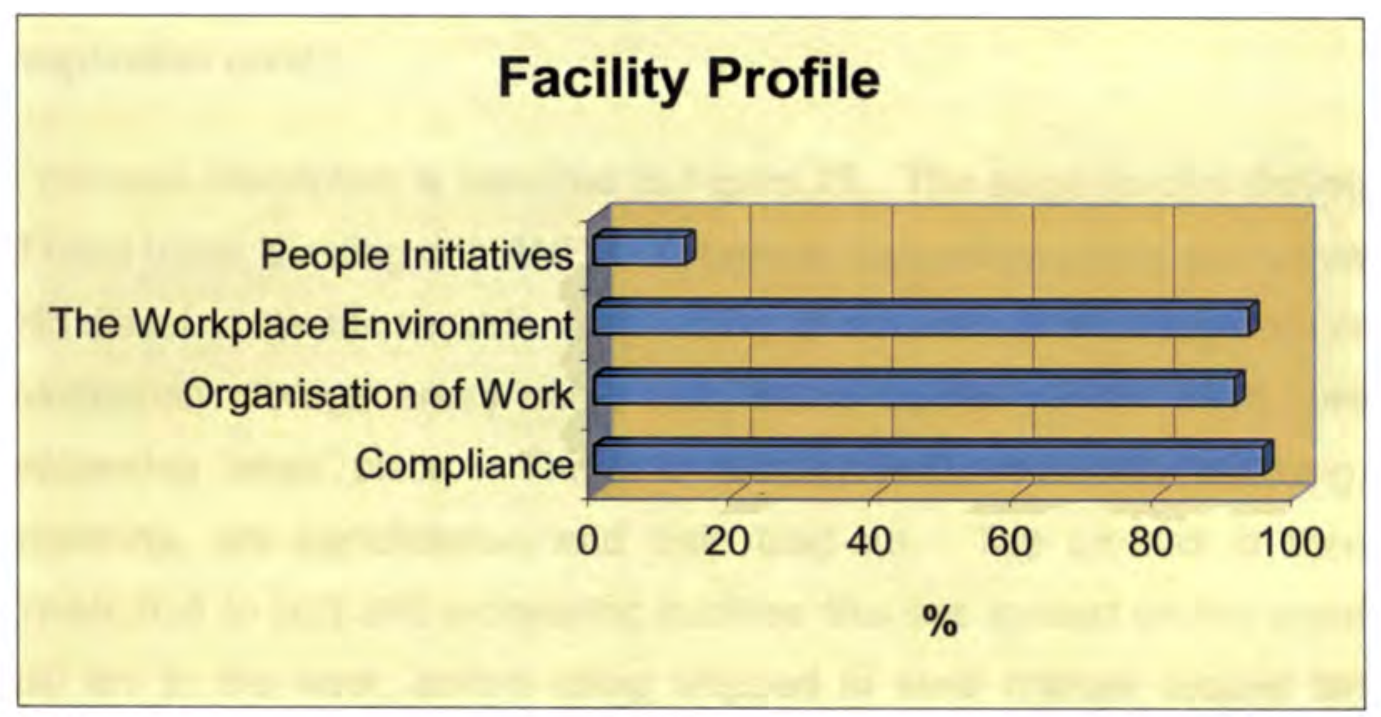

Figure 26: Facility profile - Facility Five 


\subsection{Facility 6}

\subsubsection{The Operation}

Facility Six was an open cut, iron ore mining operation in the Pilbara Region of Western Australia, 1200 kilometres from Perth. The principal mine is $5 \mathrm{~km}$ long and $2 \mathrm{~km}$ wide and is the largest single-pit iron ore mine in the world. Production at the main mine is supplemented by output from a number of smaller, adjacent mines, known as satellite ore bodies.

The principal waste material is mine rock waste and net acid-generating rock (pyritic shale). Unwanted fine materials are transferred to evaporation ponds.

A process description is provided in Figure 27. The steps involve driling of blast holes, blasting with ANFO explosives, excavation of ore and waste with diesel or electric shovels, and hauling of materials to crushing plant or overburden storage areas using rear dump trucks. From there, ore processing takes place. There is primary and secondary crushing, screening, ore beneficiation and train load-out. The product is then transported to port and processing facilities that are located on the coast $420 \mathrm{~km}$ to the west, before being shipped to steel makers around the world. 

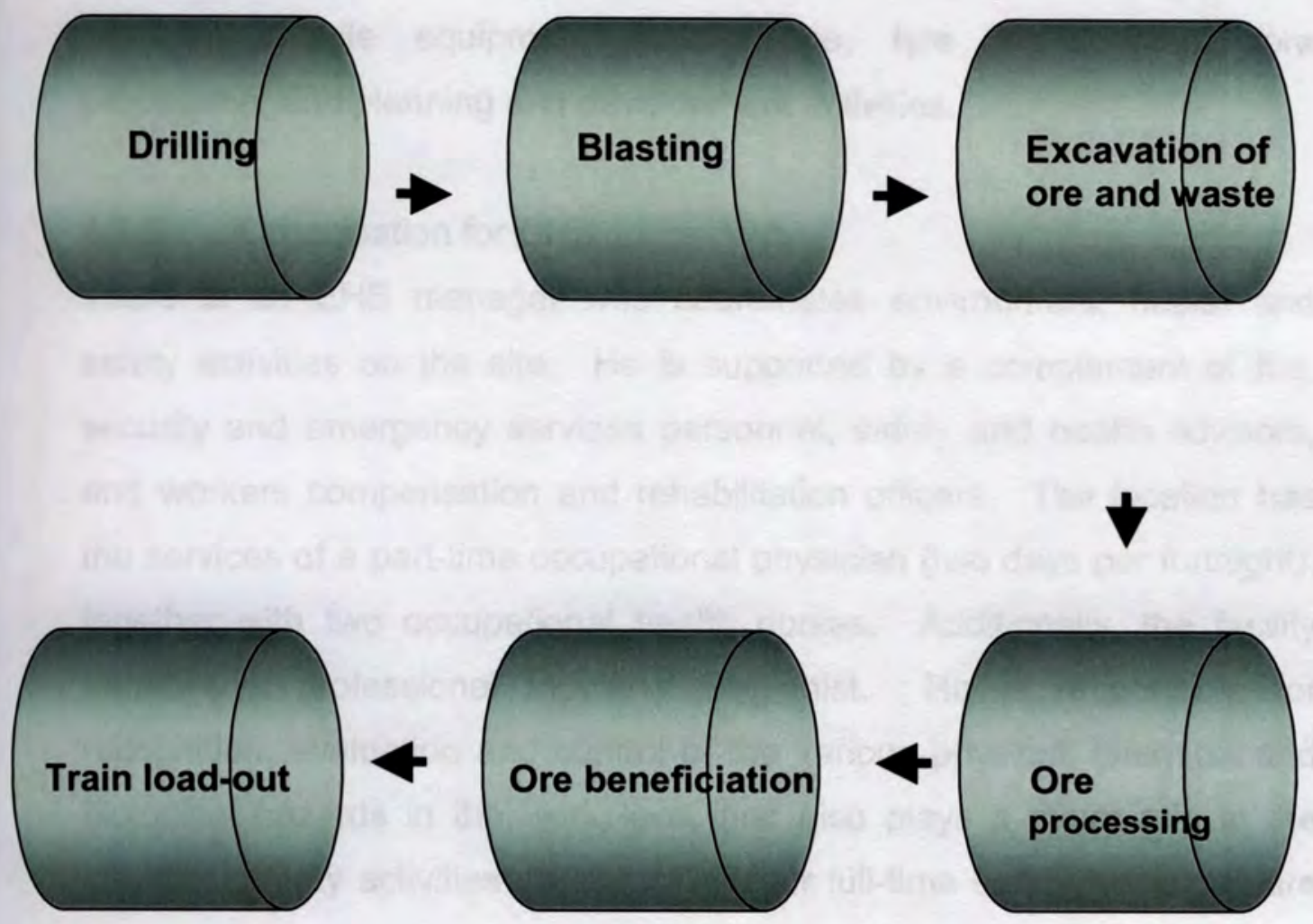

\section{Transport to port facility}

Figure 27: Schematic flowsheet - Open cut mining of hematite ore (Facility Six)

\subsubsection{The Workforce}

The workforce numbers 622 full-time, permanent employees. Company employees are divided between 211 in the administration and management group, and 411 wages employees in production and maintenance jobs (271 and 140 respectively). Most of the workforce is on a rotating shift pattern (12 hour shifts or 12.5 hour shifts), with a four-day 
on, four-day off rotation. There are 12 contracting organisations on-site. Collectively, these employ 133 people - mostly engaged in blasting services, mobile equipment maintenance, tyre management, ore processing, and planning and development activities.

\subsubsection{Organisation for OHS}

There is an EHS manager who coordinates environment, health and safety activities on the site. He is supported by a complement of fire, security and emergency services personnel, safety and health advisors, and workers compensation and rehabilitation officers. The location has the services of a part-time occupational physician (two days per fortnight), together with two occupational health nurses. Additionally, the facility employs a professional industrial Hygienist. $\mathrm{He}$ is responsible for recognition, evaluation and control of the various physical, chemical and biological hazards in the workplace, and also plays a major role in the chemical safety activities on site. The four full-iime safety resources are allocated to the various departments. They are supported in their efforts by a strong safety representative system (about 25 on site) - drawn from the ranks of each natural work group.

\subsubsection{Principal OHS Issues and Chalienges}

- Physical hazards: Fires, vehicle-vehicle collisions, vehicle-pedestrian collisions, struck-by (falling object) accidents, pit wall failure, electrocution, working at heights, blasting, structural failure, noise, vibration and thermal stress.

- Chemical hazards: Iron ore dust, welding fumes, sulphur dioxide (from pyritic shales), chlorine gas, asbestos fibres (from building materials), cleaning agents containing acids and alkalies, solvent-based cements, cleaners and paints, battery acid, corrosion inhibitor, epoxy resins, rubber cleaning and vulcanising compounds and biocides. There are approximately 450 chemicals on site (and in the database).

- Biological hazards: Potable water quality and legionelia.

- Ergonomic hazards: Key issues are associated with operation of hand tools such as rattle-guns in mobile equipment workshops. Office 
ergonomics is another area. The seat change-out policy for heavy mobile equipment has overcome past problems with seat-related musculo-skeletal injury.

\subsubsection{Other Relevant Information}

The facility belongs to an international metal business that covers different segments, from mining of iron ore through to manufacture of steel. As such, it obtains leverage and benefits from the OHS knowledge, systems, procedures, tools and resources that reside within the wider organisation.

Performance expectations cascade down from the divisional level to the site and, ultimately, through to the individual. A "Road Map to Success" publication defines the process. Some responsibilities have a statutory basis. The roles of Registered Mine Manager and Ventilation Officer would be examples of this.

The Department of Minerals and Petroleum Resources conducted an audit of management systems at Facility Six in September 2001. The site obtained a score of $89.0 \%$ - based on the structure described by DMPR (2002) and allided to earlier. Figures provided by the State Mining Engineer (M. Knee, personal communication, 2003) indicate that 128 mine sites and mineral processing operations in Western Australia have been audited in this way over the past five years. Scores ranged from $35 \%$ to $99 \%$, with a mean of $76 \%$.

In 2002, the site experienced a Lost Time Injury Frequency Rate of 0.50 per $1,000,000$ hours worked.

\subsubsection{Organisational Effectiveness}

The questions posed in Booklet 2, under the heading "general OHS", are designed to provide a preliminary gauge of organisational effectiveness. The location representative responded in the affirmative to all nine questions. Thus, the facility has in place:

- A clearly expressed OHS policy.

- A philosophy and commitment to exceed minimum standards. 
- Integration of OHS into the key business functions of planning, organising, leading, controlling and evaluating.

- Clearly defined OHS performance expectations and measures for all levels in the organisation.

- Injury management plans.

- Clear evidence of management involvement and commitment to OHS.

- Clearly assigned OHS responsibilities for managers and supervisors, expressed in terms of their normal duties. These are found in Position Descriptions and in Key Performance Indicators.

- Commitment and involvement of employees, with formal and informal structures to encourage their participation.

- A generally held view within management that Corporate investment in OHS is good business practice.

\subsubsection{Benchmarking, Best Practice and Technology Transfer}

The location OHS Superintendent indicated that previous efforts with respect to external OHS benchmarking have been of a very limited nature. He mentioned an activity that took place with a provider of behavioural safety training. The provider compared site $f$ igrams with what was being offered by its organisation and made some suggestions for change to the site efforts. However, this doesn't fit the benchmarking criteria and attributes that have been described in this study.

The site participates in a Corporate OHS audit program that is based on the wider organisation's 15-part HSE standards. There is also a self-audit (critical element) effort that is applied regularly at the site.

Apparently, the facility has not been involved in structured technology transfer activities for OHS.

\subsubsection{Self-Ranking in OHS}

The location OHS representative thought that his site would rate in the top five percent of sites within the Mining Industry of Western Australia. He based this on his knowledge of systems and procedures in place at his site, together with the low incidence of chemical-related injury and near- 
miss experience at the facility. He also had some anecdotal information from his external professional network and from suppliers and others who move from site to site.

\subsubsection{Potential Benchmarking Partners}

The Osboume Metals and Century Zinc organisations in Queensland were put forward as the companies most admired in terms of performance and reputation for excellence in OHS. Both have figured prominently in recent rounds of the MINEX awards, as presented by the Minerals Council of Australia, and described earlier.

\subsubsection{Chemical Safety Preliminary Questionnaire}

In relation to the cnemical safety questionnaire presented in Part $B$ of Booklet 2, the location representative responded to most elements in the affirmative. Thus, the facility is characterised by the following:

- A published policy on the management of hazardous materials.

- Roles and responsibilities have been clearly assigned.

- A current hazardous materiais inventory is available.

- Material Safety Data Sheets (MSDSs) are available for every chemical entering or leaving the site.

- An MSDS will normaliy precede the first supply of a hazardous material to the location.

- There is interaction between the Purchasing and Safety/Health groups to screen new materials requested for purchase. Approval is based on a desk-top review and/or an assessment in the workplace.

- There is a system to prevent non-approved chemicals coming on-site and for ensuring that those chemicals that are on-site have been approved.

- There is no formal system to track where and how much chemical usage occurs on-site.

- The site complies with all relevant legal requirements for the safe storage, transport, issue, use and disposal of hazardous materials employed or generated by the on-site operations. 
- Labels and warning signs are posted for the more important hazardous materials used on-site or sent elsewhere from the site.

- Contractors are expected to meet similar requirements and standards with respect to the hazardous materials they bring onto the site. They are required to provide a list of chemicals that they wish to use at the location and need to address this aspect in the contractor safety plan.

\subsubsection{Flow Chart}

There are several controls built into the process for procurement of hazardous substances. These cover access through the Stores requisition system, by purchase requisition and by direct ordering through a Supply catalogue of approved hazardous materials. Purchases by petty cash or credit card, or use of free samples are, in theory, not available without authorisation by the Supply Department or the Site Industrial Hygienist. This is a grey area. The annual chemical audit program is one of the measures used to identify any unauthorised chemicals that might have found their way onto the site.

Figure 28 represents a simplified version of the steps used at Facility Six to vet and control access of new chemicals to the site. 


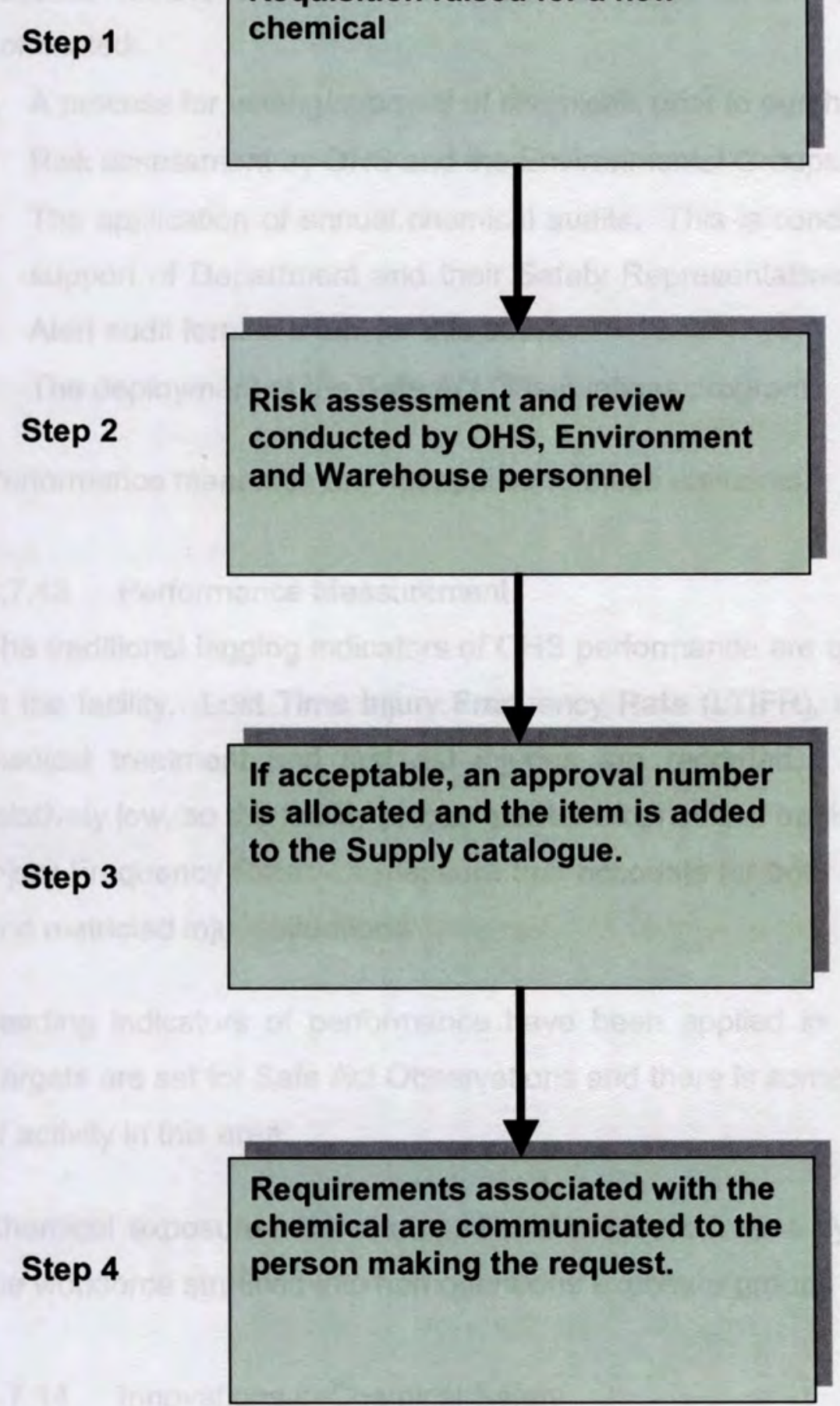

Figure 28: Acquisition of new chemicals - Facility Six 


\subsubsection{Critical Success Factors}

The site representative was asked to nominate the four most critical success factors in relation to chemical safety at the location. He nominated:

- A process for vetting/approval of chemicals prior to purchase or use.

- Risk assessment by OHS and the Environmental Groups.

- The application of annual chemical audits. This is conducted with the support of Department and their Safety Representatives. The Chem Alert audit form is a tool for this activity.

- The deployment of the Safe Act Observations program.

Performance measures are not applied to these elements.

\subsubsection{Performance Measurement}

The traditional lagging indicators of OHS performance are quite prominent at the facility. Lost Time Injury Frequency Rate (LTIFR), restricted duty, medical treatment and first aid injuries are recorded. The LTIFR is relatively low, so the facility places greater emphasis in tracking "Classified Injury Frequency Rate ${ }^{n}$ - a measure that accounts for both lost work days and restricted injury situations.

Leading indicators of performance have been applied in a limited way. Targets are set for Safe Act Observations and there is some measurement of activity in this area.

Chemical exposures are managed and measured via a system that has the workforce stratified into homogeneous exposure groups (HEGs).

\subsubsection{Innovations in Chemical Safety}

The location was able to demonstrate several innovations and practices that could be of potential interest to other organisations.

Details are provided in the sections that follow but, in brief, these cover:

- Checks that are applied as to the quality of MSDSs from suppliers and distributors. A chemical will not be approved for use on site unless it 
has a MSDS that complies with the National Code of Practice for the Preparation of Material Safety Data Sheets.

- Use of Homogeneous Exposure Groups (HEGs) to stratify exposures at the site.

- Risk mitigation initiatives.

- Substitution initiatives - such as replacement of hydrocarbon-based degreasing solvents with environmentally-friendly alternatives.

- Employee involvement and communication efforts.

- Medical surveillance.

\subsubsection{Compliance}

This element was examined with the aid of Booklet 3 (Appendix 7) and the Western Australian Mines Safety and Inspection Regulations 1995.

- Reg. 7.21: The Material Safety Data Sheets (MSDSs) held on site are in the Worksafe Australia format and are current (by definition, less than 5 years old). The sheets are provided by the organisation Chem Alert, an external party that specialises in this area. They are made available to employees in both hard copy and electronic forms. Additionally, held on site is a compilation of original, hard copy MSDSs from suppliers - to meet statutory requirements.

- Reg. 7.22: Containers are generally of suitable design and manufacture. One of the items on the inspection checklist addresses the issue of correct labelling of containers that hold decanted materials.

- Reg. 7.23: Many of the more substantial containers that are used on site are returned to the supplier and are then recycled. Containers for lubricants and detergents were cited as examples. Those that remain are disposed of to a secure landfill site.

- Reg. 7.24: Materials coming onto the site appear to be labelled in accordance with the Australian Dangerous Goods Code. The Chem Alert system enables labels to be printed out for use when chemicals at the site are transferred into small containers.

- Reg. 7.25: MSDS registers are well developed and maintained. MSDS information is freely accessible to employees and the register 
contains details of risk assessments carried out in accordance with the reguiations.

- Reg. 7.26: Work procedures, rules and training are applied to ensure that persons working in enclosed spaces, such as chutes, drums and sumps, are aware of the potential risks attached to the use of hazardous substances in those spaces.

- Reg. 7.27: Qualitative and quantitative risk assessments have been carried out, as appropriate, and are formally documented. This is part of the approval process.

- Reg. 7.28: The hierarchy of controls is well understood and deployed on the site. Suitable procedures and arrangements apply to the application of engineering, administrative and work practice (systems of work) controls, and personal protective equipment controls. Respiratory protective equipment is subject to fit testing procedures.

- Reg. 7.29: Atmospheric monitoring programs are under the direction of a professional Industrial Hygienist and are well developed. Most, but not all, exposure levels are below those identified in Worksafe Australia's Exposure Standards. The facility applies the DOIR Guideline on Extended Workshifts to ensure that measured exposure levels are adjusted to account for the additional period that people are at work (beyond eight hours).

- Reg. 7.30: Two site nurses carry out the health surveillance programs at the site. A part-time occupational physician acts in support. Health assessments are carried out at initial employment and every two years thereafter.

- Reg. 4.24-4.29: The site appears to meet regulatory requirements relating to provision of appropriate equipment, facilities and services for first aid. The occupational health nurses, emergency services officers and other individuals with first aid training provide a good 24-hour coverage in this area. Safety showers are strategically located around the site.

- Reg. 4.13: The site appears to meet all regulatory requirements for training of employees and the maintenance of training records. 


\subsubsection{Organisation of Work}

This element was examined with the aid of Booklet 4 (Appendix 7). Twenty-two lines of inquiry were pursued under the principal headings of leadership, commitment, planning, organisation, training, communication and measurement. Table 30 below provides a summary of the response from Facility Six to the questions raised in the booklet. Self-ratings fall into four categories - Poor $(P)$, Fair $(F)$, Good $(G)$ or Excellent $(E)$. The interviewee rated eighteen of the twenty-two elements as "Good" or "Excellent", thereby activating follow-up questions about key initiatives and success factors. Elements allocated a "Fair" rating in the table that follow are usually associated with programs or efforts that are still at an early stage of development or are carried out sporadically. 
Table 30

Organisation of Work - Facility Six

No. Element $\quad \begin{gathered}\text { Self- } \\ \text { Rating }\end{gathered} \quad$ Key OHS Initiatives and Success Factors

1. Management visibility

2. Policy enunciation

3. Tracking performance

4. Financial resources

5. Internal resources

6. External resources
Exc. Senior management leadership is evident at safety meetings. Visibility is achieved via demonstrated leadership, through personal example, by promoting initiatives and by frequent site inspections and reviews.

Good Wall-mounted policy statements are found in many well-accessed plant locations.

Policy statements feature in many of the location's publications. OHS

Representatives are used to enunciate and deliver key initiatives.

Good Specific, measurable OHS activities are included in performance plans and appraisal systems for all employees. Expectations are outlined in position descriptions and key performance indicators (KPIs).

Exc. Departmental budgets carry provision for local OHS initiatives, such as training, equipment, engineering and capital works, and the facility has a sound track record in terms of expenditure.

Exc. There is strong leadership and support from the Corporate Office. This drives a lot of change at the site level. Line management and OHS Representatives are important resources for the OHS effort.

Fair 


No. Element $\quad \begin{gathered}\text { Self- Key OHS Initiatives and Success Factors } \\ \text { Rating }\end{gathered}$

7. Employee involvement

8. Strategic planning

9. Target-setting

10. Contractor plans

11. Organisational structure

12. OHS integration

13. Departmental integration

14. Induction training

15. Refresher training
Good There is strong employee involvement in departmental and crew level safety activities

Fair

Exc. Corporate goals, targets and performance indicators are set, documented, communicated, monitored and reviewed annually.

Good Contractors submit a Safety Management Plan for approval. These are reviewed as part of pre-qualification checks.

Exc. The organisational structure encourages interaction of $\mathrm{OHS}$ professionals with line management and shop floor personnel.

Exc. Site and departmental OHS plans are integrated with broader Corporate plans.

Good Safety consultants and health professionals are assigned to support particular Departments.

Exc. Induction is structured around a half-day site program and a half-day departmental program.

Good The "Driving in Mining" program is applied widely. There is a theory and practical component to this annual exercise. 


No. Element $\begin{gathered}\text { Self- } \\ \text { Rating }\end{gathered} \quad$ Key OHS Initiatives and Success Factors

16. Safety rep training Exc. Safety Representatives receive a standard, accredited 5-day introductory course. Supervisors receive a three-day version of this. SGIO is the training provider.

17. Effectiveness of Fair training

18. Hazard communication

Good Many approaches are used in this area - such as tool-box talks, delivery at crew safety meetings and safety briefs (hard-copy and electronic versions).

19. Communication of

Good Workplace agreements at the site include a targets performance expectations element. There is linkage between broad corporate business plans through to individual performance plans.

20. Delivery of feedback Good There are many channels for feedback - from "toolbox" meetings at the shop floor level through to electronic and hard copy reports, newsletters, six-monthly "State of the Nation" presentations, and similar.

21. Use of lagging indicators

Good The system is centred on the reporting of lost time injuries, restricted injuries, medical treatments and first aid injuries. Classified Injury Frequency Rate is the principal measure. This is well deployed and understood.

22. Use of leading indicators 


\subsubsection{Hazard Recognition}

This element was examined with the aid of Booklet 5 (Appendix 7). Three lines of inquiry were pursued via questions on walk-through surveys (an established technique for identifying hazards, evaluating work practices and checking the efficacy of existing controls), job dictionaries (that itemise jobs and tasks, as a preliminary to assignment of exposure information and the assessment of risk) and chemical inventories. Table 31 below provides a summary of the response from Facility Six to the questions raised in the booklet. Self-rating categories are as described earlier. The interviewee rated one of the sub-elements as "Good", thereby activating follow-up questions about key initiatives and success factors for that item.

Table 31

Hazard Recognition - Facility Six

Self- Key OHS Initiatives and Success

Rating

Factors

1. Walk-through surveys Good The Site Hygienist conducts these on a regular basis. Departmental inventories are checked against chemicals found. The workforce is stratified into Homogeneous Exposure Groups (HEGs) for Industrial Hygiene purposes.

2. Job dictionaries

Not

used

3. Chemical inventories

Fair

\subsubsection{Risk Assessment}

This element was also examined with the aid of Booklet 5 (Appendix 7). Six lines of inquiry were pursued via questions on the existence and 
effectiveness of sampling plans and schedules, qualitative assessment programs, quantitative assessment programs, statistical treatment of sampling results, exposure baselines and record-keeping. Table 32 below provides a summary of the response from Facility Six to the questions raised in the booklet. Self-rating categories are as described earlier. The interviewee rated four of the sub-elements as "Good", thereby activating follow-up questions about key initiatives and success factors.

\section{Table 32}

Risk Assessment - Facility Six

\begin{tabular}{ccc} 
No. Element & $\begin{array}{c}\text { Self- } \\
\text { Rating }\end{array}$ & Key OHS Initiatives and Success \\
& Factors \\
\hline
\end{tabular}

1. Sampling plans

2. Qualitative assessments

3. Quantitative assessments

4. Statistical treatment

5. Exposure baselines

6 Record-keeping
Fair

Fair

Good Personal exposure assessments are directed at a workforce that has been stratified into Homogeneous Exposure Groups (HEGs).

Good "LogNorm2" analysis is applied to sample results. The site has found this to be helpful in identifying key areas for attention.

Good Exposure baselines have been established for particulate contaminants.

Good An MS Access Database is used to store sampling data.

\subsubsection{Risk Control}

This element was also examined with the aid of Booklet 5 (Appendix 7). Six lines of inquiry were pursued via questions on processes for feasibility 
assessment and priority setting (for OHS controls), together with the effectiveness of substitution/elimination activity, procurement controls, engineering controls, administrative/work practice controls and personal protective equipment controls. Table 33 below provides a summary of the response from Facility Six to the questions raised in the booklet. Selfrating categories are as described earlier. The interviewee rated four of the sub-elements as "Good", thereby activating follow-up questions about key initiatives and success factors.

\subsubsection{Industrial Hygiene Program Development}

This element was also examined with the aid of Booklet 5 (Appendix 7). Eleven characteristics accompany a Stage 1 (beginning) program, thirteen are used to describe a Stage II (improving) program and twenty are used to describe a Stage III (advanced) program.

Based on the criteria used in Booklet 5, Facility Five has an "improving" industrial hygiene program, with some elements of "advanced".

\subsubsection{People Initiatives}

This element was examined with the aid of Booklet 6 (Appendix 7). Eight lines of inquiry were pursued via questions on the existence and effectiveness of behavioural safety programs, task observation programs (a component within a broader behavioural safety program), motivation and recognition programs, attitude surveys, morale surveys, safety (management) contact programs, compliance surveys relating to the use of personal protective equipment and site promotional efforts for OHS. Table 34 below provides a summary of the response from Facility Six to the questions raised in the booklet. Self-rating categories are as described earlier. The interviewee rated five of the eight sub-elements as "Good", thereby activating follow-up questions about key initiatives and" success factors attached to these. 
Table 33

Risk Control - Facility Six

\section{Self-}

No.

Rating Key OHS Initiatives and Success Factors

1. Feasibility assessments

Fair

2. Elimination/substitution

Good There are several current examples of successful elimination and substitution effort - for example, use of environmentally-friendly degreasing agents, elimination of $A C$ sheeting and similar.

3. Procurement controls Good IH and Supply personnel interact formally when vetting new chemicals proposed for use on the site.

4. Engineering controls

Fair

5. Work practice controls

Good

Systems of work have been developed for key hazardous materials, such as chlorine, liquid nitrogen, dry ice and similar.

6. PPE controls

Good These are supported by supervisory practices, together with training and awareness packages.

There is ready availability and deployment of PPE conforming to Australian Standards, and meeting comfort and practicability criteria. A PPE Committee assists with arrangements for selection, use, fitting and storage. 


\begin{tabular}{lcc}
\hline No. Element & $\begin{array}{c}\text { Self- } \\
\text { Rating }\end{array}$ & Key OHS Initiatives and Success \\
& Factors \\
\hline
\end{tabular}

1. Behavioural safety

Good The Safe Act Observation (SAO) Program is the centrepiece for this work. This involves Management and the workforce and is supported by well-defined processes and materials.

2. Task observation

3. Motivation/recognition

4. Attitude surveys

5. Morale surveys

6. Management contact

7. PPE compliance

8. Promotional efforts
Good As above.

Fair

Fair

Fair

Good

The Safe Act Observation Program includes a management contact element. The Vice President (Mining) and his reporting staff are active in this effort.

Good Compliance with personal protective equipment requirements is one of the items examined as part of the Safe Act Observation Program.

Good Promotional efforts are based on verbal and non-verbal messages (such as that provided by senior management involvement in the SAO Program). Signage and notice board initiatives are also prominent. 


\subsubsection{Summary}

The results for Facility Six provided above may be represented as the following scores:

- The overall score for the compliance element was $95 \%$.

- The overall score for the organisation of work (or management enabler) element was $82 \%$.

- The overall score for the workplace environment (or technical initiatives) element was $67 \%$.

- The overall score for the people initiatives element was $62 \%$.

Industrial Hygiene program development was characterised as "improving" and assigned a score of $85 \%$. The latter was based on working through program elements identified in Booklet 5 .

33 potential benchmarking opportunities emerged from the process applied to Facility Six in this study. 18 of these relate to organisation of work (management enablers), 10 to the workplace environment (technical initiatives) and the remaining five to people initiatives.

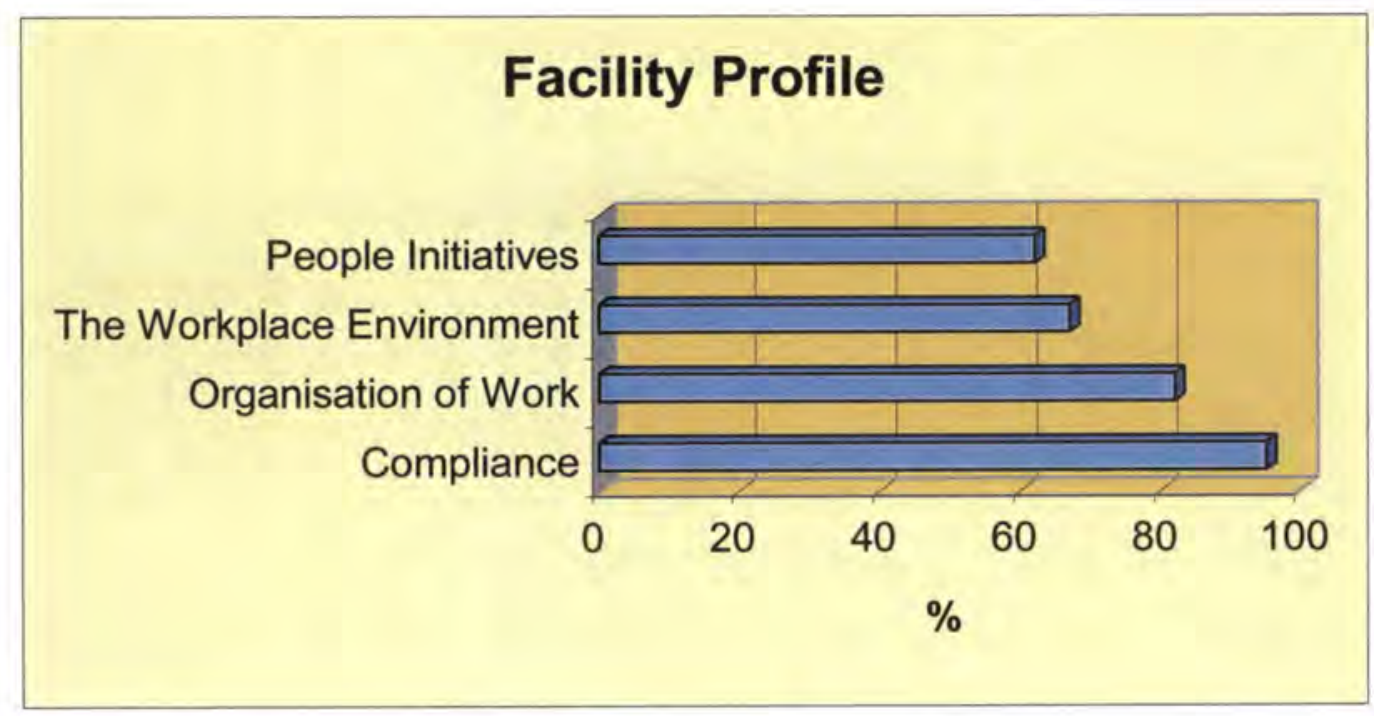

Figure 29: Facility profile - Facility Six 


\subsection{Facility Seven}

\subsubsection{The Operation}

Facility Seven was a gold mining, milling and extraction operation in the Kalgoorlie District of Western Australia, some $600 \mathrm{~km}$ east of Perth and approximately $30 \mathrm{~km}$ south of Kalgoorlie. The facility is owned by the same organisation that operates Facility Five, described earlier. Thus, it would be expected that many of the management systems and approaches would be similar. The complex has three open cut pits, one underground operation and two gold processing plants. Underground mining, above ground mining, drilling and other services are carried out by contractors. However, the client organisation carries out its own gold extraction via two similar Carbon-in-Leach (CIL) processing plants that are about $7 \mathrm{~km}$ apart. The main raw materials for the gold plants are sodium cyanide, caustic soda, hydrochloric acid, lime, LPG and liquid oxygen. The end product is gold bullion.

There are no housing or messing facilities on-site and most employees commute from the towns of Kalgoorlie and Kambalda.

A simplified description of the CIL Process is provided in Figure 30. 


\section{Ore from Mine}

\section{\& Tailings Deposit}
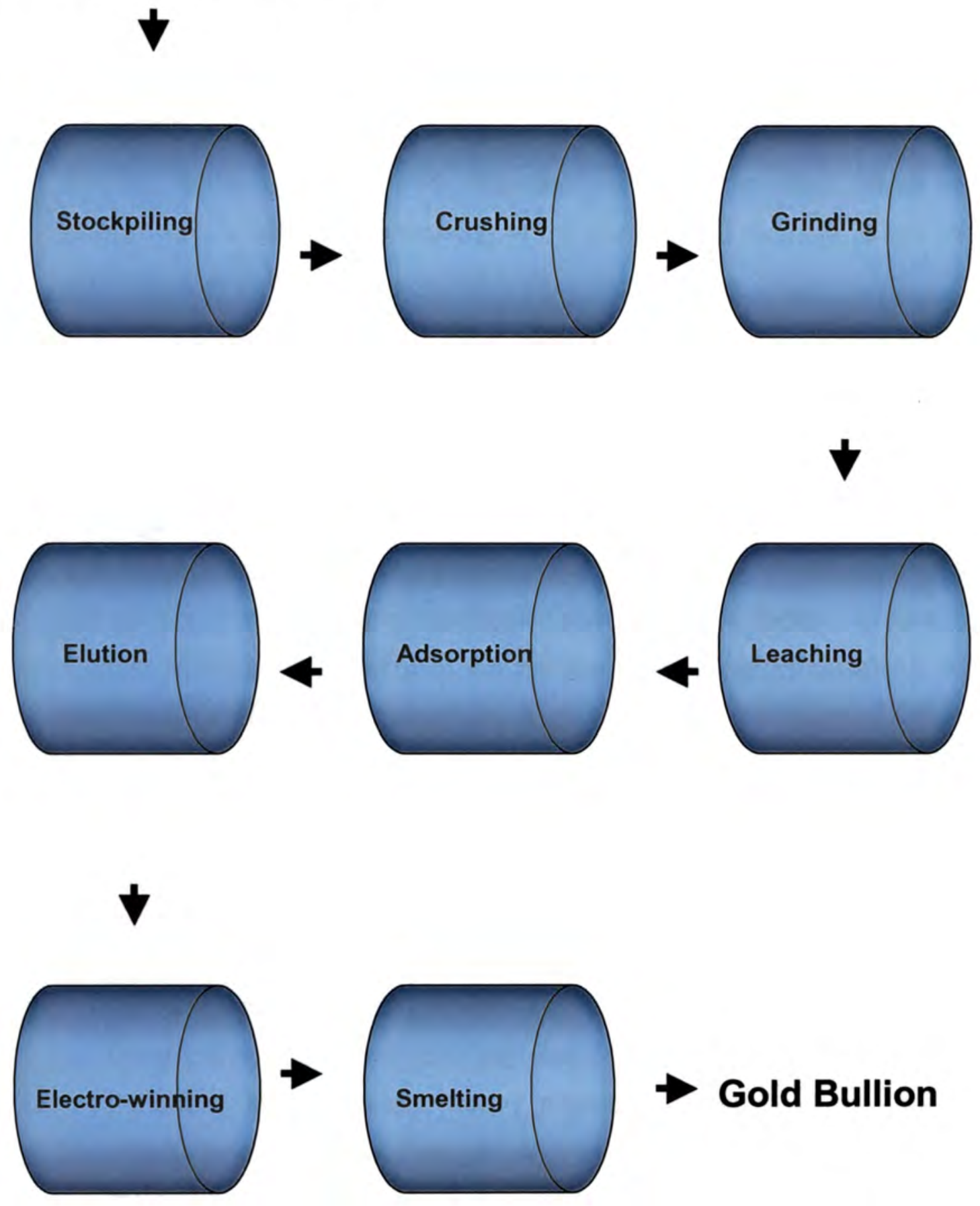

$\rightarrow$ Gold Bullion

Figure 30: Schematic flowsheet - ore to gold bullion (Facility Seven) 


\subsubsection{The Workforce}

There are 98 full-time, permanent Company employees at the site - 11 in administrative and mines rescue-type roles, 8 in exploration, 19 in surface mining roles and 60 engaged in various tasks at the two gold treatment plants. There are four main contracting organisations on site and dozens of smaller ones. These are engaged in operations such as underground mining, surface mining and diamond drilling. In total, there are approximately 350 people working at the complex. The mine is run on a continuous shift basis and several rosters are in use.

\subsubsection{Organisation for OHS}

The location has a Loss Control Manager and a Loss Control Administrator. The former reports functionally to a Group Loss Control Manager who has broader corporate responsibilities. As with the sister site in the Murchison District (Facility Five), there is an emphasis on firstaid training and emergency response capability at the facility. The Loss Control Manager interacts with the various contracting organisations that carry out work for her company.

\subsubsection{Principal OHS Issues and Challenges}

- Physical hazards: Noise, vibration, ionising radiation, and thermal stress were examples in the health-related area. Also, there are hazards attached to the vehicle/pedestrian interface, work at heights, falling objects and electrical work.

- Chemical hazards: Gold-bearing ore dust, tailings dust, alkaline materials, acid materials, heavy metals, welding fume, combustion gases and industrial chemicals (garnet-based abrasive blasting materials, LPG, oxygen and other compressed gases, cleaning agents, oils and greases, surfactants, adhesives, sealants, solvents, spray paint, insecticides, herbicides and similar). A recent risk assessment report identified two major chemical-related sources of risk. These were the entering of leach or adsorption tanks without use of proper tank entry procedures and potential exposure to hazardous 
substances, such as hydrogen cyanide, during delivery operations and maintenance of storage facilities.

- Biological hazards: None apparent.

- Ergonomic hazards: Lifting, pushing, pulling, twisting, awkward postures, repetitive motion and similar have potential to produce ergonomic injury.

\subsubsection{Other Relevant Information}

The facility changed hands last year and now belongs to an international mining operation. It benefits from the OHS knowledge and experience within the wider organisation. The site also draws from the procedures and systems that were available to it under previous ownership arrangements.

Two years ago, the Department of Minerals and Petroleum Resources of Western Australia foreshadowed its intention to conduct an audit of management systems at the site. Preparations were made, but the visit did not eventuate. Thus, this source of external validation of study materials is not availabie for this site.

In 2002, the site experienced a Lost Time Injury Frequency Rate of 7.9 per $1,000,000$ hours for all employees (including contractors) and a 6.3 rate for Company employees only.

\subsubsection{Organisational Effectiveness}

The questions posed in Booklet 2, under the heading "general OHS", are designed to provide a preliminary gauge of organisational effectiveness. The location representative responded in the affirmative to all nine questions. Thus, the facility has in place:

- A clearly expressed OHS policy.

- A philosophy and commitment to exceed minimum standards.

- Integration of OHS into the key business functions of planning. organising, leading, controlling and evaluating. 
- Clearly defined OHS performance expectations and measures for all levels in the organisation.

- Injury management plans.

- Clear evidence of management involvement and commitment to OHS.

- Clearly assigned OHS responsibilities for managers and supervisors, expressed in terms of their normal duties.

- Commitment and involvement of employees, with formal and informal structures to encourage their participation.

- A generally held view within management that Corporate investment in OHS is good business practice.

\subsubsection{Benchmarking, Best Practice and Technology Transfer}

The location representative indicated that previous efforts with respect to external OHS benchmarking have been of a very limited nature - of a type characterised by site visits and unstructured discussions on a few items of interest. This was in keeping with findings from the other sites in the study. Technology transfer does occur, but usually does not involve shop floor personnel.

\subsubsection{Self-Ranking in OHS}

The location representative drew a distinction between OHS management systems as developed and their deployment. With regard to the former, she thought that her sice would rate in the top $10 \%$ of sites within the Mining indusiry of Western Australia. However, s:ie noted that, while management systems were comprehensive, deployment was not strong and allocated this to the category "top $75 \%$, but not top half". She based the rating on her knowledge of systems and procedures in place at her site and within the wider organisation.

\subsubsection{Potential Benchmarking Partners}

The location representative nominated the DuPont organisation as most admired in terms of performance and reputation for excellence in OHS. She said that her present role did not give her much exposure to other operations. 


\subsubsection{Chemica! Safety Preliminary Questionnaire}

In relation to the chemical safety questionnaire presented in Part B of Booklet 2, the location representative responded to ali but one of the elements in the affirmative. The first item, dealing with policy for chemical safety, is covered in broad terms under the OHS policy statement and is not dealt with separately. Thus, the facility is characterised by the following:

- Roles and responsibilities have been clearly assigned. The Loss Contro: Manager is the "Chemical Controller" for the site.

- A current hazardous materials inventory is available.

- Material Safety Data Sneets (MSDSs) are available for every chemical entering or leaving the site.

- An MSDS will normally accompany the first supply of a hazardous material to the location.

- There is interaction between the Purchasing and Safety/Health groups to screen new materials requested for purchase.

- There is a system to prevent non-approved chemicals coming on-site and for ensuring that those chemicals that are on-site have been approved.

- There is a system to track where and how much chemical usage occurs on-site.

- The site complies with all relevant legal requirements for the safe storage, transport, issue, use and disposal of hazardous materials employed or generated by the on-site operations.

- Labels and warning signs are posted for every hazardous material used on-site or sent elsewhere from the site.

- Contractors are expected to meet similar requirements and standards with respect to the hazardous materials they bring onto the site.

\subsubsection{Flow Chart}

The purchasing arrangements at Facility Seven are relatively simple. The range of chernicals used is modest and the Loss Control Manager reviews and approves all requests for new chemicals. 
Figure 31 represents a simplified version of the steps used at Facility Seven to vet and control access of new chemicals to the site.

Step 2

Purchase Requisition raised,

Step 1 together with a" request to trial/use" form (for all new chemicals)

The Site Loss Control Manager reviews chemicals and usages on site, and then approves the item.

Step 3

\section{Adjustments made to the Chemical} Register and Chem Alert database.

Step 4

Item received at the site. The Stores Officer advises the Loss Control Manager of this. The chemical is deployed for use

Figure 31: Acquisition of new chemicals - Facility Seven 


\subsubsection{Critical Success Factors}

The site representative was asked to nominate the four most critical success factors in relation to chemical safety at the location. She nominated:

- Use of the Chem Alert materials information service.

- Adherence to rules and procedures.

- Use of a system to address ordering, vetting, supply and distribution of hazardous materials.

- Effective deployment of personal protective equipment.

\subsubsection{Performance Measurement}

The traditional lagging indicators of OHS performance are quite prominent at the facility. There is a close focus on Lost Time Injury Frequency Rate (LTIFR) - defined as injury resulting in at least one complete lost shift. Medical Treatment injuries (MTIs) are tracked - these relate to injuries requiring treatment by a doctor but not resulting in a lost shift. A third measure is referred to as Minor Injury (MI). This covers injuries that require first aid treatment only.

Leading indicators of performance take the form of workplace inspection outcomes, task observations, pre-start checks, workplace checklists, job safely analyses (although mostly limited to shut-down situations), induction and on-going training records, pre-shift meetings and safety meetings.

\subsubsection{Innovations in Chemical Safety}

The location was able to demonstrate several strengths that could be of potential interest to other organisations. Most relate to aspects of OHS that are broader than chemical safety.

Details are provided in the sections that follow but, in brief, these cover:

- The Safety Management System

- The "Whole of Mining Risk Assessrient".

- Emergency response.

- Site induction training programs besed on use of CD-Rom tools. 
- Accident investigation.

\subsubsection{Compliance}

This element was examined with the aid of Booklet 3 (Appendix 7) and the Western Australian Mines Safety and Inspection Regulations 1995.

- Reg. 7.21: The Material Safety Data Sheets (MSDSs) held on site are in the Worksafe Australia format and are current (by definition, less than 5 years old). In the main, an external party that specialises in this area provides these (Chem Alert). Hard copy versions are kept in crisis management lockers, in the mill control room, and in office and workshop areas.

- Reg. 7.22: Containers are generally of suitable design and manufacture. There was some variability around the site in terms of the practices surrounding the transfer of bulk supplies to more suitable smaller containers.

- Reg. 7.23: Many of the more substantial containers that are used on site are returned to the supplier and are then recycled. Those that remain are disposed of to a local landfill site. Stores personnel manage this activity.

- Reg. 7.24: Materials coming onto the site appear to be labelled in accordance with the Australian Dangerous Goods Code. However, there appears to be some variability in practices attached to the use of labels when transferring chemicals from supplier-provided containers to smaller, site-issued containers.

- Reg. 7.25: MSDS information is freely accessible to employees. There was some dubiety about content of the MSDS register as it relates to the inclusion of risk assessments and details of duties that may give rise to exposure. There is an annual audit of chemicals on site and the register is updated if any anomalies are found.

- Reg. 7.26: Work procedures, permits, rules and training are applied to ensure that persons working in enclosed spaces are aware of the potential risks attached to the use of hazaidous substances in those spaces. 
- Reg. 7.27: Qualitative and quantitative risk assessments have been carried out, as appropriate, and are formally documented.

- Reg. 7.28: The hierarchy of controls is suitably understood at a management level but is more problematic at a shop floor level. There are some opportunities for improvement in relation to the application of engineering, administrative and work practice controls, and personal protective equipment controls.

- Reg. 7.29: Atmospheric monitoring programs are carried out by an external organisation. Most, but not all, exposure levels are consistently below those identified in Worksafe Australia's Exposure Standards.

- Reg. 7.30: Health assessments are carried out for pre-employment purposes. Thereafter, efforts are mainly directed at meeting the requirements of the Govemment's Mine Health program. Biological monitoring (for blood lead levels) is carried out on a six-monthly basis with workers in the gold room and laboratory.

- Reg. 4.24-4.29: The site appears to meet regulatory requirements relating to provision of appropriate equipment, facilities and services for first aid.

- Reg. 4.13: The site appears to meet all regulatory requirements for training of employees and the maintenance of training records.

\subsubsection{Organisation of Work}

This element was examined with the aid of Booklet 4 (Appendix 7). Twenty-two lines of inquiry were pursued under the principal headings of leadership, commitment, planning, organisation, training, communication and measurement. Table 35 below provides a summary of the response from Facility Seven to the questions raised in the booklet. Self-ratings fall into four categories - Poor, Fair, Good or Excellent. The interviewee rated six of the twerity-two elements as "Good" or "Excellent", thereby activating follow-up questions about key initiatives and success factors. Elements allocated a "Fair" rating in the table that follow are usually associated with programs or efforts that are still at an early stage of development or are carried out sporadically. 


No. Element $\begin{gathered}\text { Self- Key OHS Initiatives and Success Factors } \\ \text { Rating }\end{gathered}$
1. Management
Fair
visibility

2. Policy enunciation Fair

3. Tracking Fair performance

4. Financial resources Poor

5. Internal resources Poor

6. External resources Good The facility contracts specialist groups to perform tasks such as chemical audits and air monitoring. Supplier organisations, such as CSBP, provide training and information packages.

\section{Employee}

Fair involvement

8. Strategic planning

9. Target-setting

10. Contractor plans

11. Organisational structure

12. OHS integration

Fair

13. Departmental integration

14. Induction training

15. Refresher training

16. Safety rep training
Poor

Good Induction is structured around a one-day MARCSTA program (generic), followed by a local/departmental program.

Fair

Good The introductory course is followed by further skills training and awarenessbuilding. 


No. Element $\quad \begin{aligned} & \text { Self- Key OHS Initiatives and Success Factors } \\ & \text { Rating }\end{aligned}$

17. Effectiveness of training Fair

18. Hazard communication Good Many approaches are used in this area. These include accident/incident report forms, hazard logs, safety alerts and notice board items, the Chem Alert database, awareness training and similar.

19. Communication of Fair targets

20. Delivery of feedback Fair

21. Use of lagging indicators Good

The system is centred on the reporting of lost time injuries, medical treatment injuries and minor injuries. This is well deployed and understood.

22. Use of leading indicators Poor

\subsubsection{Hazard Recognition}

This element was examined with the aid of Booklet 5 (Appendix 7). Three lines of inquiry were pursued via questions on walk-through surveys (an established technique for identifying hazards, evaluating work practices and checking the efficacy of existing controls), job dictionaries (that itemise jobs and tasks, as a preliminary to assignment of exposure information and the assessment of risk) and chemical inventories. Table 36 below provides a summary of the response from Facility Seven to the questions raised in the booklet. Self-rating categories are as described earlier. The interviewee rated one of the sub-elements as "Good", thereby 
activating follow-up questions about key initiatives and success factors for that element.

Table 36

Hazard Recognition - Facility Seven

Self- Key OHS Initiatives and Success

No.

Element

Rating

Good The Loss Control Manager conducts

1. Walk-through surveys these. Departmental inventories are checked against chemicals found. The underground operations tend to be the local benchmark for this activity.

2. Job dictionaries

Poor

3. Chemical inventories

\subsubsection{Risk Assessment}

This element was also examined with the aid of Booklet 5 (Appendix 7). Six lines of inquiry were pursued via questions on the existence and effectiveness of sampling plans and schedules, qualitative assessment programs, quantitative assessment programs, statistical treatment of sampling results, exposure baselines and record-keeping. Table 37 below provides a summary of the response from Facility Seven to the questions raised in the booklet. Self-rating categories are as described earlier. The interviewee rated three of the six sub-elements as "Good" or "Excellent", thereby activating follow-up questions about key initiatives and success factors. 


\begin{tabular}{ccc} 
No. Element & $\begin{array}{c}\text { Self- } \\
\text { Rating }\end{array}$ & Key OHS Initiatives and Success \\
& Factors \\
\hline
\end{tabular}

1. Sampling plans

Good These are established on an annual basis. Essentially, these respond to CONTAM requirements.

2. Qualitative assessments

Poor

3. Quantitative assessments

Good

Some useful assessment approaches are being applied to work in confined spaces.

4. Statistical treatment

Poor

5. Exposure baselines

Poor

6 Record-keeping
Exc. This is built around the "Site Safe" recording and tracking systems.

\subsubsection{Risk Control}

This element was also examined with the aid of Booklet 5 (Appendix 7). Six lines of inquiry were pursued via questions on processes for feasibility assessment and priority setting (for OHS controls), together with the effectiveness of substitution/elimination activity, procurement controls, engineering controls, administrative/work practice controls and personal protective equipment controls. Table 38 below provides a summary of the response from Facility Seven to the questions raised in the booklet. Selfrating categories are as described earlier. The interviewee rated three of the sub-elements as "Good" or "Excellent", thereby activating follow-up questions about key initiatives and success factors. 


\section{Self-}

No. Element Rating Key OHS Initiatives and Success Factors

1. Feasibility assessments Good A formal process is deployed to rank projects in terms of their importance and in terms of the feasibility of achieving a successful outcome.

2. Elimination/substitution Fair

3. Procurement controls Exc. The Loss Control Manager interacts with Purchasing and Stores functions when dealing with new chemicals and personal protective equipment controls proposed for use on the site.

4. Engineering controls Fair

5. Work practice controls Fair

6. PPE controls Good There is ready availability and deployment of PPE conforming to Australian Standards, and meeting comfort and practicability criteria.

\subsubsection{Industrial Hygiene Program Development}

This element was also examined with the aid of Booklet 5 (Appendix 7). Eleven characteristics accompany a Stage I (beginning) program, thirteen are used to describe a Stage II (improving) program and twenty are used to describe a Stage III (advanced) program.

Based on the criteria used in Booklet 5, Facility Seven has a "beginning" industrial hygiene program, with some elements of "improving". 


\subsubsection{People Initiatives}

This element was examined with the aid of Booklet 6 (Appendix 7). Eight lines of inquiry were pursued via questions on the existence and effectiveness of behavioural safety programs, task observation programs (a component within a broader behavioural safety program), motivation and recognition programs, attitude surveys, morale surveys, formal safety (management) contact programs, compliance surveys relating to the use of personal protective equipment and site promotional efforts for $\mathrm{OHS}$. Table 39 below provides a summary of the response from Facility Seven to the questions raised in the booklet. Self-rating categories are as described earlier. None of the elements attracted a "Good" or "Excellent" rating from the interviewee.

Table 39

People Initiatives - Facility Seven

\begin{tabular}{cc} 
No. Element & $\begin{array}{c}\text { Self- } \\
\text { Rating }\end{array}$ \\
\hline
\end{tabular}

1. Behavioural safety Not used

2. Task observation Not used

3. Motivation/recognition Poor

4. Attitude surveys Not used

5. Morale surveys Not used

6. Formal management Not used contact programs

7. PPE compliance Not used

8. Promotional efforts Poor

\subsubsection{Summary}

The results for Facility Seven are represented in Figure 32 as the following scores:

- The overall score for the compliance element was $94 \%$. 
- The overall score for the organisation of work (or management enabler) element was $28 \%$.

- The overall score for the workplace environment (or technical initiatives) element was $45 \%$.

- The overall score for the people initiatives element was $0 \%$.

Industrial Hygiene program development was characterised as "beginning", with some elements of "improving". It was assigned a score of $68 \%$, based on which of the program elements identified in Booklet 5 appear to be in place.

Thirteen potential benchmarking opportunities emerged from the process applied to Facility Seven in this study. Six of these relate to organisation of work (management enablers) and seven to the workplace environment (technical initiatives). Most relate to systems and procedures.

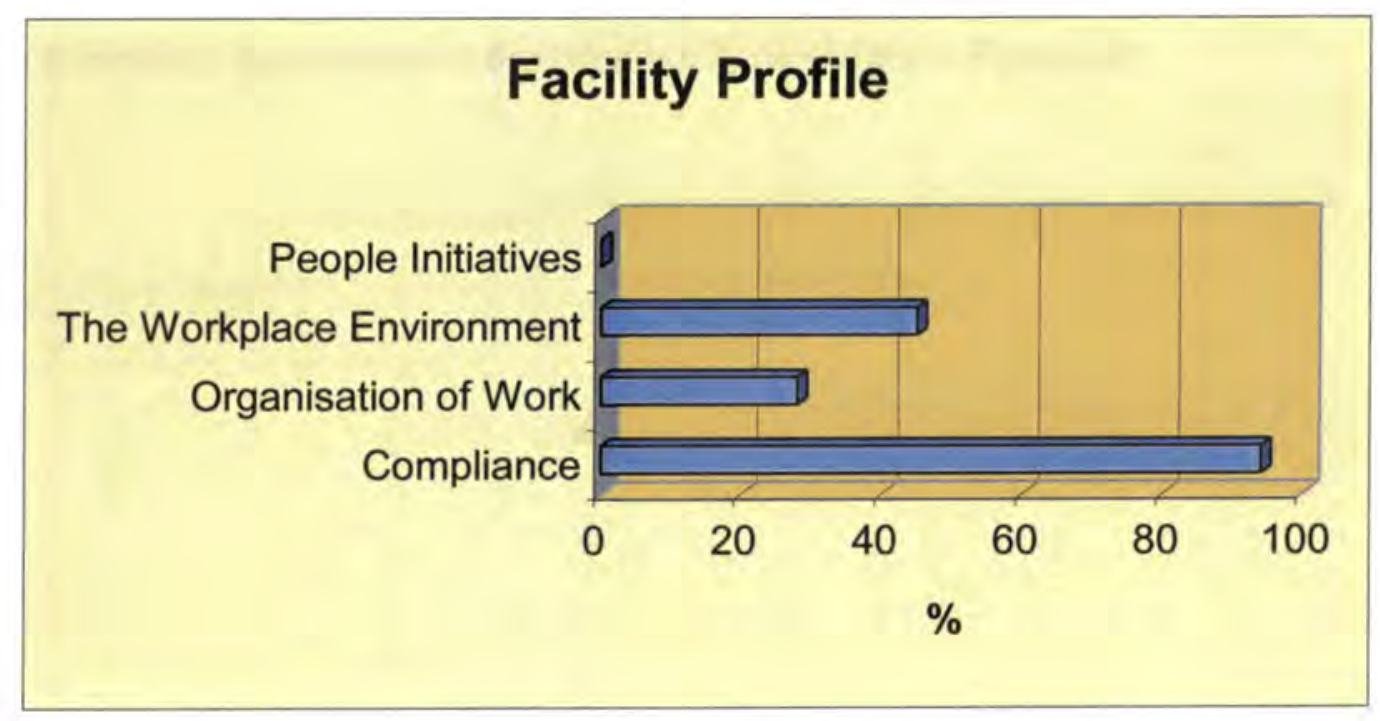

Figure 32: Facility profile - Facility Seven 


\subsection{Facility Elght}

\subsubsection{The Operation}

Facility Eight was a nickel refinery located in the Kwinana industrial zone. Refining is the last stage in the nickel mining and beneficiation process. It follows the mining, concentrating and smelting stages, all of which occur at other Company facilities in Western Australia. The main raw materials for the facility are nickel matte, ammonia, steam, hydrogen, hydrogen suphide and sulphuric acid. The main end products from the facility are nickel briquettes and nickel powder (both containing $99.8 \%$ nickel). Overall, about $60 \%$ of the Company's nickel production is fully processed through to metal. The upstream concentrating and smelting activities that are conducted elsewhere produce nickel concentrate (containing about $20 \%$ nickel) and nickel matte (containing about $72 \%$ nickel and 5\% copper). The refinery also produces a range of intermediate, saleable products chiefly, copper sulphide, mixed sulphides (of nickel and cobalt) and ammonium sulphate.

The principal waste material is a residue containing iron oxide.

A simplified description of the process is provided in Figure 33. 
Nickel matte

from Smelter
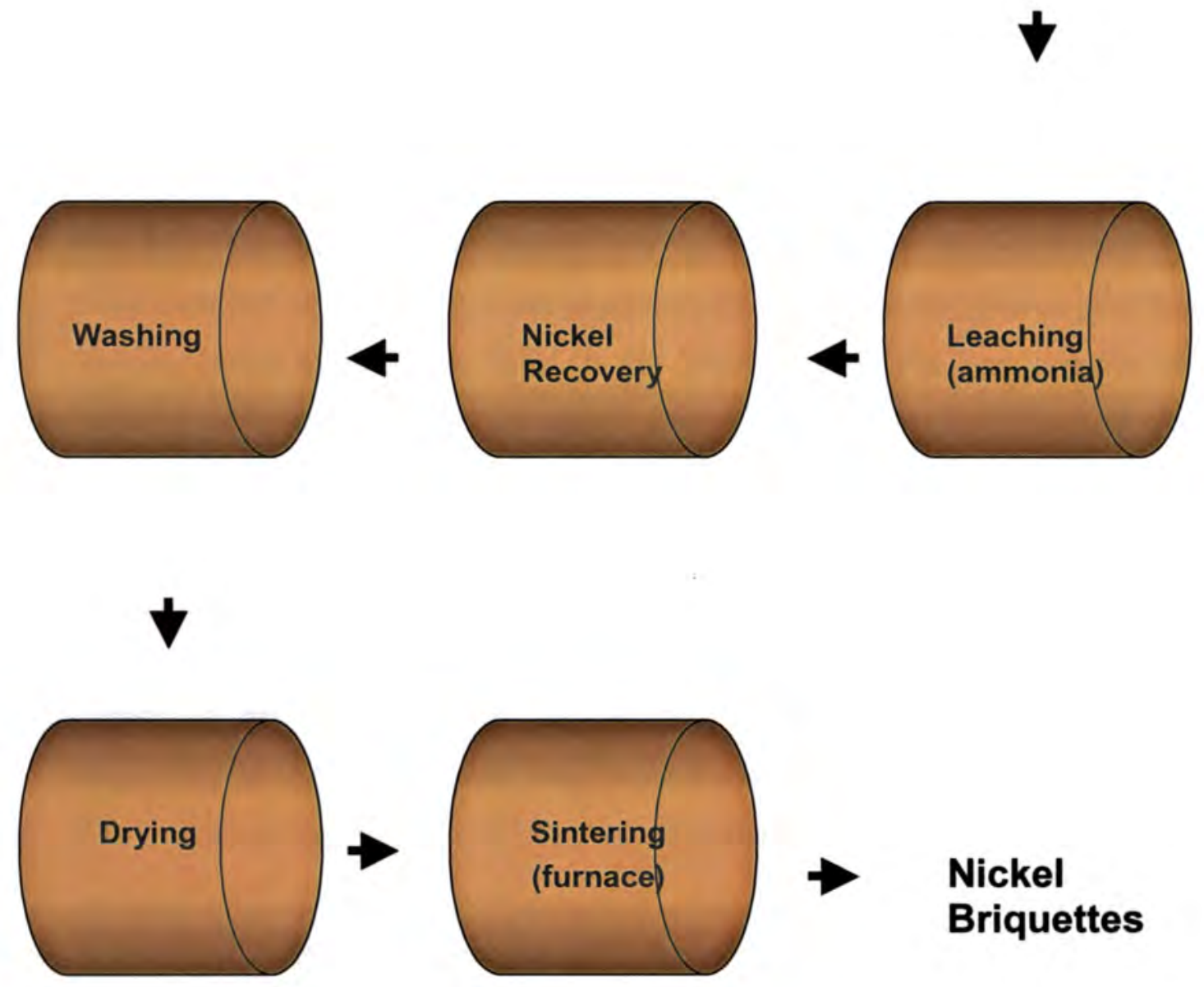

$\rightarrow \quad$ Nickel

\section{Briquettes}

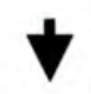

\section{Nickel Powder}

Figure 33: Schematic flowsheet - nickel refining operations (Facility Eight)

\subsubsection{The Workforce}

The workforce numbers 218 full-time, permanent employees. Company employees are split between 88 in the administration and management group, and 130 operation-based employees in production and 
maintenance jobs. Most of the workforce is on a rotating shift pattern (12 hour shifts). There are seven principal contracting organisations on-site, with a combined workforce of about 74. These people are engaged mostly in maintenance activities involving scaffolding, welding, painting and insulation work. The nickel-based slurries at the site are highly corrosive and abrasive, so there is a lot of attention to application of protective surface coatings.

The location has a very low rate of labour turnover $(2 \%$ in 2002$)$ and there are many employees with more than 10 years of service at the site.

\subsubsection{Organisation for OHS}

The location employs a part-time occupational physician and a part-time nurse. These individuals report to the Senior Safety Advisor, who in tum reports to the General Manager. The Environmental Coordinator is responsible for environmental and industrial hygiene activities at the site and he is supported by two people who carry out most of the hygienerelated tasks at the site. The Coordinator reports to the Business Development Manager, who in turn reports to the General Manager. There is an emphasis on first-aid training and emergency response capability at the site. Some shop floor individuals have been selected as "safety pillar representatives" and act in support of site safety initiatives.

\subsubsection{Principal OHS Issues and Challenges}

- Physical hazards: Noise, vibration, thermal stress, electrical hazards, work at heights, and hazards associated with prevalence of high pressure, high temperature slurries.

- Chemical hazards: There are about 450 chemicals on site. Some of the more important are hydrogen, ammonia, carbon dioxide, nitrogen, hydrogen sulphide, sulphuric acid, sodium hydroxide, welding gases, water treatment chemicals, isocyanates, oils and greases, solvents, surface coatings, sealants, grouts, curing agents, adhesives, nickel slurries and arsenic trioxide. Many of the tanks at the facility are used for mixing operations. 
- Biological hazards: Bio-aerosols associated with cooling water operations.

- Ergonomic hazards: Lifting, pushing, pulling, twisting, awkward postures, repetitive motion and similar have potential to produce ergonomic injury.

\subsubsection{Other Relevant Information}

The facility belongs to a major mining house and, as such, obtains leverage and benefits from the OHS knowledge, systems, procedures, tools and resources that reside within the wider organisation.

The Department of Minerals and Petroleum Resources sonducted an audit of management systems at Facility Eight in Febriary 1998. The site obtained a score of $66 \%$ - based on the structure described by DMPR (2002) and alluded to earlier. Figures provided by the State Mining Engineer (M. Knee, personal communication, 2003) indicate that 128 mine sites and mineral processing operations in Western Australia have been audited in this way over the past five years. Scores ranged from $35 \%$ to $99 \%$, with a mean of $76 \%$.

In 2002, the site experienced a Lost Time Injury Frequency Rate of 6.1 per $1,000,000$ hours worked.

\subsubsection{Organisational Effectiveness}

The questions posed in Booklet 2, under the heading "general $\mathrm{OHS}^{n}$, are designed to provide a preliminary gauge of organisational effectiveness. The location representative responded in the affirmative to all nine questions. Thus, the facility has in place:

- A clearly expressed OHS policy.

- A philosophy and commitment to exceed minimum standards.

- Integration of OHS into the key business functions of planning, organising, leading, controlling and evaluating.

- Clearly defined OHS performance expectations and measures for all levels in the organisation. 
- Injury management plans.

- Clear evidence of management involvement and commitment to OHS.

- Clearly assigned OHS responsibilities for managers and supervisors, expressed in terms of their normal duties.

- Commitment and involvement of employees, with formal and informal structures to encourage their participation (although variable across the site).

- A generally held view within management that Corporate investment in OHS is good business practice.

\subsubsection{Benchmarking, Best Practice and Technology Transfer}

The location representative indicated that the broader organisation has been involved in several types of technical exchange in the past. These have covered safety, health and environmental matters. He cited examples of interaction under the auspices of the wider nickel industry and some reciprocal visits to other sites within the Western Australian Mining Industry.

The facility is seen as a benchmark within the broader organisation to which it belongs. For example, its Management of Change system has become a corporate standard. The site takes part in formal OHS audit efforts, with these involving both internal and external validation.

\subsubsection{Self-Ranking in OHS}

The location representative thought that his site would rate in the top five percent of sites within the Mining Industry of Western Australia. He based this on his knowledge of systems and procedures in place at his site, together with the low incidence of chemical-related injury and near-miss experience at the facility. He also had some anecdotal information from his external professional network and from suppliers and others who move from site to site.

\subsubsection{Potential Benchmarking Partners}

The DuPont organisation was put forward as the company most admired in terms of performance and reputation for excellence in OHS. Within 
Western Australia, the Alcoa mining and refinery operations were seen as a benchmark.

\subsubsection{Chemical Safety Preliminary Questionnaire}

In relation to the chemical safety questionnaire presented in Part B of Booklet 2, the location representative responded to all but one of the elements in the affirmative. The first item, dealing with policy for chemical safety, is covered in broad terms under the OHS policy statement and is not dealt with separately. Thus, the facility is characterised by the following:

- Roles and responsibilities have been clearly assigned.

- A current hazardous materials inventory is available.

- Material Safety Data Sheets (MSDSs) are available for every chemical entering or leaving the site.

- An MSDS will normally accompany the first supply of a hazardous material to the location.

- There is interaction between the Purchasing and Safety/Health groups to screen new materials requested for purchase.

- There is a system to prevent non-approved chemicals coming ori-site and for ensuring that those chemicals that are on-site have bein approved.

- There is a system to track where and how much chemical usage occuis on-site (larger items only).

- The site complies with all relevant legal requirements for the safe storage, transport, issue, use and disposal of hazardous materials employed or generated by the on-site operations.

- Labels and warning signs are posted for hazardous material used onsite or sent elsewhere from the site (more so for the latter).

- Contractors are expected to meet similar requirements and standards with respect to the hazardous materials they bring onto the site. 


\subsubsection{Flow Chart}

There are several controls built into the process for procurement of hazardous substances. The requisition writer is required to complete a hazardous material risk assessment form when use of a new chemical is being proposed. The Environmental Coordinator is the appointed hazardous substances coordinator and he plays the key role in assessing whether the material may be used on site. There is little opportunity for purchases to occur via use of petty cash or credit cards, and access of free samples does not occur without authorisation by the Environmental Coordinator. Figure 34 represents a simplified version of the steps used at Facility Eight to vet and control access of new chemicals to the site. 


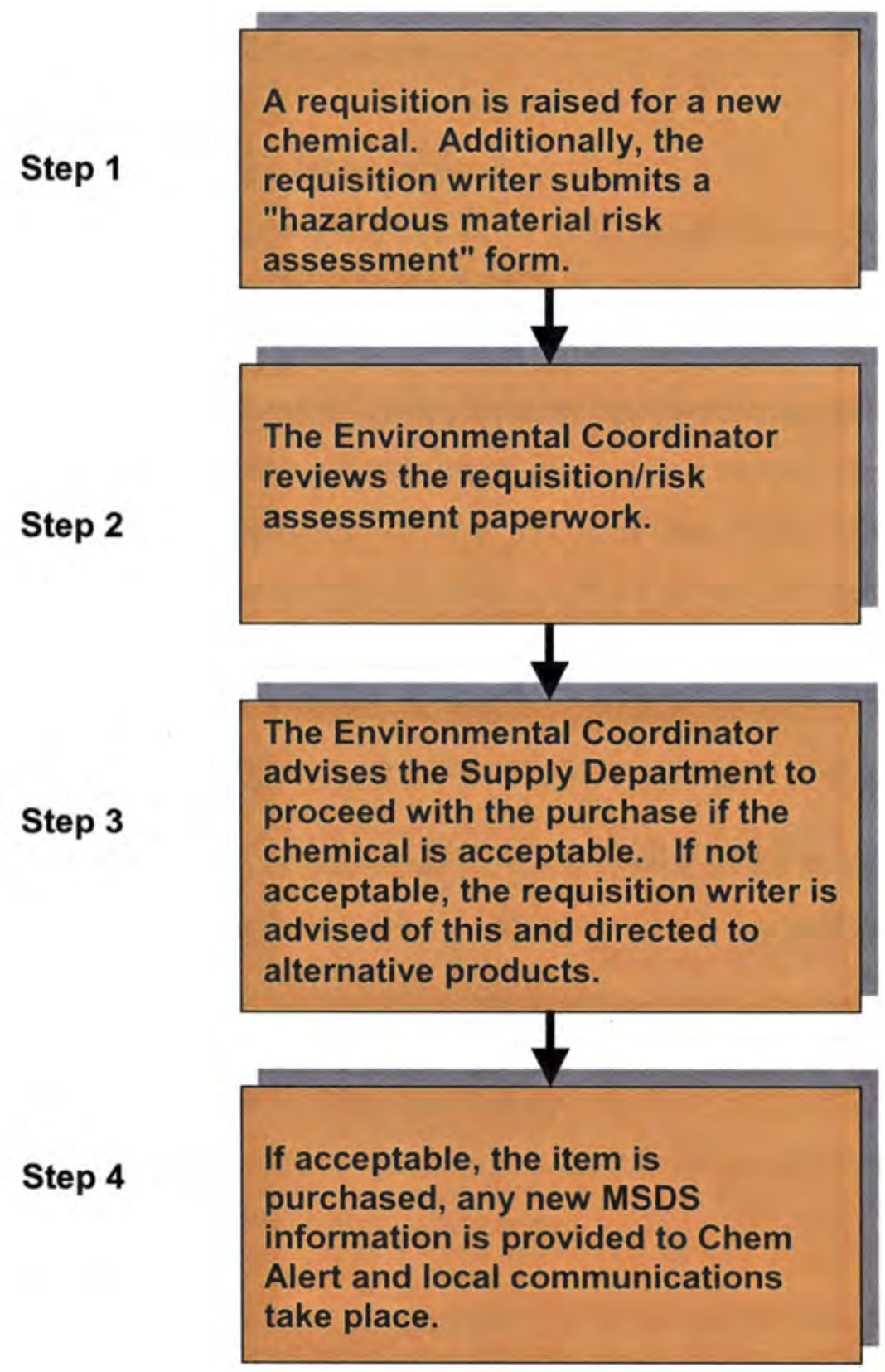

Figure 34: Acquisition of new chemicals - Facility Eight

\subsubsection{Critical Success Factors}

The site representative was asked to nominate the four most critical success factors in relation to chemical safety at the location. He nominated:

- Inventory control.

- Emergency response and waste management.

- Storage and handling arrangements and procedures. 
- Training and communication.

\subsubsection{Performance Measurement}

The traditional lagging indicators of OHS performance are prominent at the facility. There is a close focus on a rate that combines Lost Time Injury Frequency Rate (LTIFR) and Medical Treatment Injuries (MTIs). This combined measure covers injıries that require some form of medical attention beyond first-aid treatment, injuries that restrict the individual from performing all elements of the job and lost time injuries. First aid treatments are tracked separately. A third measure used on the site is called Serious Potential Injury (SPI) - to cover those incidents that have potential to cause a fatality or result in serious disability. When an SPI is raised, this triggers a series of investigation, analysis and reporting steps.

Leading indicators of performance have been applied to some extent. The STOP audit program is applied vigorously to identify problem areas and to drive change.

\subsubsection{Innovations in Chemical Safety}

The location was able to demonstrate several innovations that could be of potential interest to other organisations.

Details are provided in the sections that follow but, in brief, these cover:

- Strategic planning processes.

- Well-deployed procedures for vetting new chemicals. This inciudes use of Hazardous Material Risk Assessment forms.

- Management of change procedures.

- Substitution/elimination initiatives.

\subsubsection{Compliance}

This element was examined with the aid of Booklet 3 (Appendix 7) and the Western Australian Mines Safety and Inspection Regulations 1995.

- Reg. 7.21: The Material Safety Data Sheets (MSDSs) held on site are in the Worksafe Australia format and are current (by definition, less 
than 5 years old). As with other study sites, it is the Chem Alert organisation that provides support to the facility to meet its MSDS obligations. There is reliance on electronic versions of the MSCS and all employees have access to a computer to facilitate this. The currency of hard copy versions may be an issue.

- Reg. 7.22: Containers are generally of suitable design aid manufacture. There was some variability around the site in terms of the practices surrounding the transfer of bulk supplies to more suitable smaller containers.

- Reg. 7.23: Many of the more substantial containers that are used on site are returned to the supplier and are then recycled. Those that remain are held temporarily beforc being unisposed of by a contractor to a secure landfill site. Alternative use of empty containers by employees is discouraged.

- Reg. 7.24: Materials coming onto the site appear to be labelled in accordance with the Australian Dangerous Goods Code. However, there appears to be some variability in practices attached to the use of labels when transferring chemicals from supplier-provided containers to smaller, site-issued containers.

- Reg. 7.25: The facility maintains an inventory of chemicals held on site and MSDS information is freely accessible to employees. This is not strictly in the form of a register and does not provide details of duties that may give rise to exposure or details of any risk assessments carried out under the Regulations.

- Reg. 7.26: Work procedures, permit to work systems and training are applied to ensure that persons working in enclosed spaces are aware of the potential risks attached to the use of hazardous substances in those spaces.

- Reg. 7.27: Qualitative and quantitative risk assessments have been applied to the more significant occupational health challenges on site. For smaller items, risk assessment is applied as part of discussion and vetting arrangements at the location.

- Reg. 7.28: The hierarchy of controls is familiar to many at the site, particularly at the supervisor level. However, emphasis is applied to 
personal protective equipment controls more so than engineering or work practice controls. Very strict controls are in place for vessel entry and work in oxygen-deficient atmospheres.

- Reg. 7.29: Atmospheric monitoring programs are under the direction of the Environmental Coordinator. Most, but not all, exposure levels are consistently below those identified in Worksafe Australia's Exposure Standards.

- Reg. 7.30: Health surveillance programs are under the direction of a part-time Occupational Physician. Health assessments are carried out at initial employment and periodically thereafter at five-yearly intervals as part of the State Government's Mine Health requirements. A comprehensive, one-off health survey of the workforce is currently underway.

- Reg. 4.24-4.29: The site appears to meet regulatory requirements relating to provision of appropriate equipment, facilities and services for first aid. There are trained Emergency Response Officers on-site, as well as 2-3 people on each shift capable of administering first aid.

- Reg. 4.13: The site appears to meet regulatory requirements for training of employees and the maintenance of training records. Notwithstanding this, some opportunities exist to apply more attention to the chernical safety component of induction and refresher training.

\subsubsection{Organisation of Work}

This element was examined with the aid of Booklet 4 (Appendix 7). Twenty-two lines of inquiry were pursued under the principal headings of leadership, commitment, planning, organisation, training, communication and measurement. Table 40 below provides a summary of the response from Facility Eight to the questions raised in the booklet. Self-ratings fall into four categories - Poor $(P)$, Fair $(F)$, Good $(G)$ or Excellent $(E)$. The interviewee rated ten of the twenty-two elements as "Good", thereby activating follow-up questions about key initiatives and success factors. Elements allocated a "Fair" rating in the table that follow are usually associated with programs or efforts that are still at an early stage of development or are carried out sporadically. 


No. Element $\quad \begin{gathered}\text { Self- } \\ \text { Rating }\end{gathered}$
Key OH Initiatives and Success Factors

1. Management visibility

2. Policy enunciation

3. Tracking performance

4. Financial resources

5. Internal resources

6. External resources

7. Employee involvement

8. Strategic planning

9. Target-setting
Good Senior site management achieves high visibility via DuPont-style STOP audits and twice-weekly area inspections.

Good The business planning process is robust. Policies, goals and values cascade down through the organisation to the departmental level. Wall-mounted policy statements are found in several well-accessed plant locations.

Good The Business Plan sets out the organisation's performance expectations and targets. These are tracked on a monthly basis.

Good A high proportion of the current capital spending at the site is directed at EHS projects.

Fair

Fair

Fair

Good

A high level of attention is applied to strategic planning for OHS. The Company's Business Plan provides clear guidance to all levels in the organisation

Good Stretch targets are developed as part of the business planning process. 
Table 40

Organisation of Work - Facility Eight (Continued)

\begin{tabular}{l} 
No. Element $\quad \begin{array}{l}\text { Self- Key OHS Initiatives and Success Factors } \\
\text { Rating }\end{array}$ \\
\hline
\end{tabular}

10. Contractor plans

11. Organisational structure

12. OHS integration

13. Departmental integration

Fair

14. Induction training

15. Refresher training

16. Safety rep training

17. Effectiveness of training

18. Hazard communication

19. Communication of targets

20. Delivery of feedback

21. Use of lagging indicators

22. Use of leading indicators

Poor

Poor

Poor

Poor

Fair
Fair

Fair

Fair
Good Contractor plans are reviewed as part of pre-qualification checks and these are integrated with those of the site. A "buddy" or mentoring system is in place with longer-term contractors.

Good The organisational structure, together with briefing sessions and meeting arrangements, encourages interaction of OHS professionals with line management and shop floor personnel.

Good Site and departmental OHS plans are integrated with broader organisation's plans. OHS is the first item on the agenda for most meetings.

Good Target setting is part of the business planning process. There are many channels for feedback - from "toolbox" meetings at the shop floor level through to electronic and hard copy reports, newsletters and other presentations. 


\subsubsection{Hazard Recognition}

This subject was examined with the aid of Booklet 5 (Appendix 7). Three lines of inquiry were pursued via questions on walk-through surveys (an established technique for identifying hazards, evaluating work practices and checking the efficacy of existing controls), job dictionaries (that itemise jobs and tasks, as a preliminary to assignment of exposure information and the assessment of risk) and chemical inventories. Table 41 below provides a summary of the response from Facility Eight to the questions raised in the booklet. None of the elements attracted a rating of "Good" or "Excellent".

\section{Table 41}

Hazard Recognition - Facility Eight

\begin{tabular}{|c|c|c|c|}
\hline & & Self- & Key OHS Initiatives and Success \\
\hline No. & Element & Rating & Factors \\
\hline
\end{tabular}

1. Walk-through surveys Fair

2. Job dictionaries Poor

3. Chemical inventories Fair

\subsubsection{Risk Assessment}

This element was also examined with the aid of Booklet 5 (Appendix 7). Six lines of inquiry were pursued via questions on the existence and effectiveness of sampling plans and schedules, qualitative assessment programs, quantitative assessment programs, statistical treatment of sampling results, exposure baselines and record-keeping. Table 42 below provides a summary of the response from Facility Eight to the questions raised in the booklet. Self-rating categories are as described earlier. The interviewee rated four of the sub-elements as "Good" or "Excellent", thereby activating follow-up questions about key initiatives and success factors. 
Table 42

Risk Assessment - Facility Eight

\begin{tabular}{lcc}
\hline No. Element & $\begin{array}{c}\text { Self- } \\
\text { Rating }\end{array}$ & Key OHS Initiatives and Success \\
& Factors \\
\hline
\end{tabular}

1. Sampling plans

Exc. An extensive baseline-monitoring program is underway (projected 1600 samples over an 18-month period). This is focused on personal sampling of employee exposure and includes a random sampling feature.

2. Qualitative assessments

Good In-house resources conduct these assessments.

3. Quantitative assessments

Good In-house resources carry out the sampling component, while an external laboratory conducts analytical work.

4. Statistical treatment

Fair

5. Exposure baselines

Fair

6 Record-keeping

Good

A data management system is being developed for this purpose. It will accommodate long-term storage of electronic records and timely retrieval of information when this is required.

\subsubsection{Risk Control}

This element was also examined with the aid of Booklet 5 (Appendix 7). Six lines of inquiry were pursued via questions on processes for feasibility assessment and priority setting (for OHS controls), together with the effectiveness of substitution/elimination activity, procurement controls, engineering controls, administrative/work practice controls and personal 
protective equipment controls. Table 43 below provides a summary of the response from Facility Eight to the questions raised in the booklet. Selfrating categories are as described earlier. The interviewee rated three of the sub-elements as "Good", thereby activating follow-up questions about key initiatives and success factors.

Table 43

Risk Control - Facility Eight

Self-

No. Element Rating Key OHS Initiatives and Success Factors

1. Feasibility assessments Good A Six Sigma process is deployed to rank projects in terms of their importance and in terms of the feasibility of achieving a successful outcome. HAZOP studies are carried out when necessary.

2. Elimination/substitution Good There are several current examples of efforts to apply elimination and substitution strategies. Some of the more hazardous substances are accessed on demand - to avoid storage issues. Alternatives are being sought for hydrogen sulphide and arsenic-based reagents.

3. Procurement controls Good The purchasing system flags potential issues in the chemical management area. There is effective interaction between the Environmental Coordinator and Supply personnel when introduction of a new chemical is being contemplated for use on the site.

4. Engineering controls Fair

5. Work practice controls Fair

6. PPE controls Fair 


\subsubsection{Industrial Hygiene Program Development}

This element was also examined with the aid of Booklet 5 (Appendix 7). Eleven characteristics accompany a Stage I (beginning) program, thirteen are used to describe a Stage II (improving) program and twenty are used to describe a Stage III (advanced) program.

Based on the criteria used in Booklet 5, Facility Eight has an industrial hygiene program that could be described as "improving, with some elements of advanced".

\subsubsection{People Initiatives}

This element was examined with the aid of Booklet 6 (Appendix 7). Eight lines of inquiry were pursued via questions on the existence and effectiveness of behavioural safety programs, task observation programs (a component within a broader behavioural safety program), motivation and recognition programs, attitude surveys, morale surveys, safety (management) contact programs, compliance surveys relating to the use of personal protective equipment and site promotional efforts for OHS. Table 44 below provides a summary of the response from Facility Eight to the questions raised in the booklet. Self-rating categories are as described earlier. The interviewee rated three of the eight sub-elements as "Good" or "Excellent", thereby activating follow-up questions about key initiatives and success factors attached to these. 


\begin{tabular}{lcc}
\hline No. Element & $\begin{array}{c}\text { Self- } \\
\text { Rating }\end{array}$ & Key OHS Initiatives and Success \\
& Factors \\
\hline
\end{tabular}

1. Behavioural safety

2. Task observation

3. Motivation/recognition

4. Attitude surveys

5. Morale surveys

\section{Good}

A recent attitude survey sought employee views on the importance of OHS and its delivery on site.

Morale aspects have been

addressed in formal job satisfaction surveys conducted in 2001 and 2003.

6. Management contact Not used (formal and documented)

7. PPE compliance

Fair

8. Promotional efforts

Exc. Initiatives include front gate displays, posters, notice board items, OHS screensavers, magazine articles and Lead Team activities. The site attaches importance to the symbolism of safety.

\subsubsection{Summary}

The results for Facility Eight provided above are represented in Figure 35 as the following scores:

- The overall score for the compliance element was $85 \%$.

- The overall score for the organisation of work (or management enabler) element was $45 \%$. 
- The overall score for the workplace environment (or technical initiatives) element was $47 \%$.

- The overall score for the people initiatives element was $38 \%$.

Industrial Hygiene program development was characterised as "improving, with some elements of advanced" and assigned a score of $85 \%$. The latter was based on those program elements identified in Booklet 5 that appear to be in place.

Twenty potential benchmarking opportunities emerged from the process applied to Facility Eight in this study. Ten of these relate to organisation of work (management enablers), seven to the workplace environment (technical initiatives) and the remaining three to people initiatives.

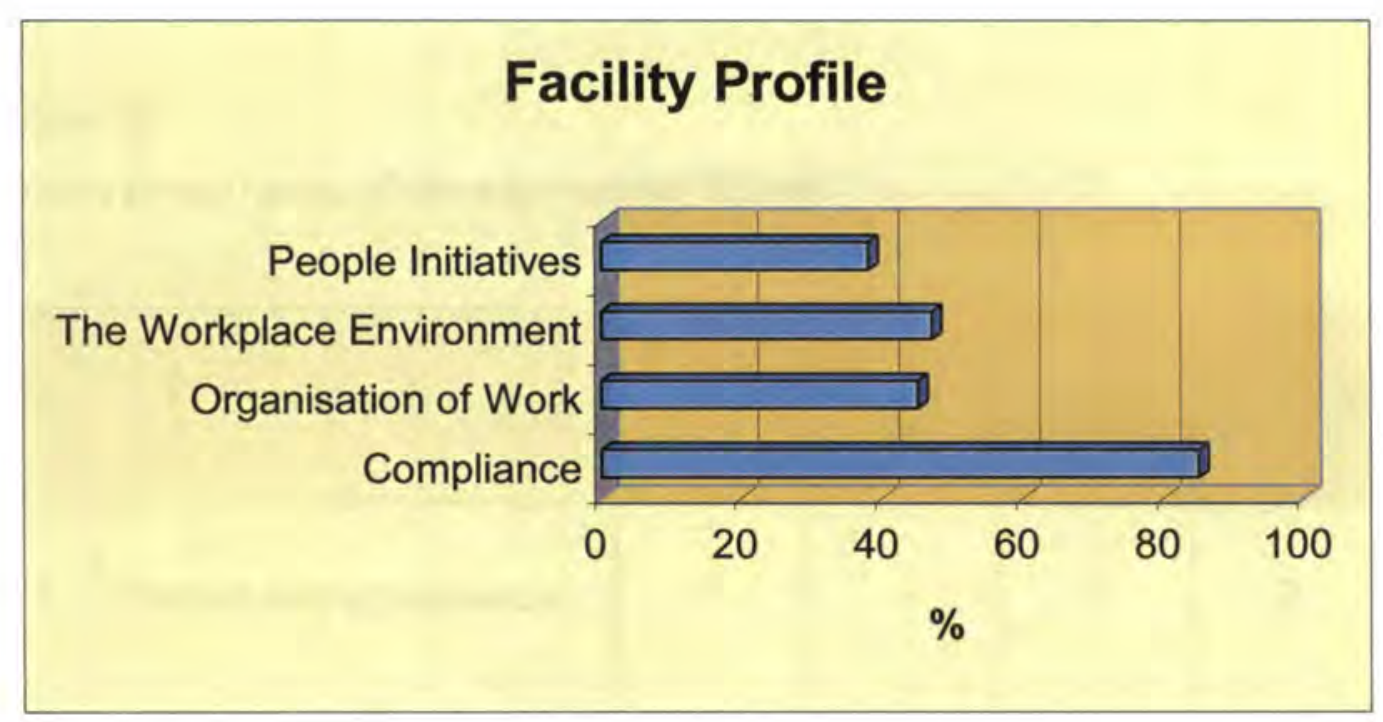

Figure 35: Facility profile - Facility Eight 
As indicated earlier, an element of the study was to assess the transferability of the study format and approach to an OHS element other than chemical safety. The booklet provided in Appendix 9 was created for this purpose. A panel of four professional physiotherapists/ergonomists and an occupational physician was then approached to rate the booklet in terms of format and appearance, clarity, ease of use and potential value as a tool for future benchmarking activity. When the same four-level scale that has been used throughout this study was applied, the booklet was rated "Good" or "Excellent" for all attributes (Table 45). The strongest element was the potential for the booklet to be applied as a tool for ergonomic exchange (four "Excellent" ratings and one "Good"). The weakest area was in relation to ease of use - suggesting some further development would be warranted for this aspect.

Table 50

Focus group rating of the ergonomics booklet

\begin{tabular}{|l|l|c|c|c|c|}
\hline No. & \multicolumn{1}{|c|}{ Criterion } & Poor & Fair & Good & Excellent \\
1. & Format and appearance & - & - & 3 & 2 \\
2. & Clarity & - & - & 2 & 3 \\
4. & $\begin{array}{l}\text { Ease of use } \\
\text { Potential as a tool for }\end{array}$ & - & - & 1 & 4 \\
\hline
\end{tabular}


One panel member commented that she thought the materials could be readily adapted to accommodate self-assessment applications for ergonomics (J. Marias, personal communication, March 23, 2003).

\subsection{Summary}

Study processes and tools have been applied in nine mining, mineral processing and related-industry sites in Western Australia. The booklet series and associated verification processes were designed to elicit and check information on how location programs and approaches fit with those advocated in the mainstream safety literature. In particular, attention was applied to identification of potential benchmarking opportunities as may exist at the facility in relation to organisation of work, the workplace environment and people initiatives. All of the study locations had strong management systems for OHS and were able to identify aspects of their operations that would be of use in a two-way benchmarking exchange.

What follows now is a discussion on the approach that was taken, the tools that were used, the outcomes from each site and various comparisons and conclusions that may be drawn from this. 


\section{CHAPTER 5 DISCUSSION}

\subsection{Overview}

This chapter deals with the logic behind the choice of various elements of management theory and the safety literature that were consulted in the preparation of the processes and tools for this study and how these elements fit together in a coherent way. Discussion is also directed at the design, delivery and evaluation of the research methodology and tools. The chapter is organised so that the results from each facility are considered in turn before any inter-site comparisons are carried out. This corresponds with the way the study was conducted. Finally, it addresses how study outcomes respond to the matters raised in the original research questions.

\subsection{Research methodology and tools}

\subsubsection{Influencing factors in the literature}

The literature review presented in Chapter Two covers many of the topics that are prominent in modem management theory and in contemporary OHS publications. The broad headings are represented in Figure 11 as an input model. What follows is an account of the rationale behind the selection of these topics and their relevance to the present study.

\section{- Quality management and continuous improvement}

Benchmarking in one form or another has existed since human beings first began exchanging ideas. It has a recognised and prominent place in modern management practice and so a logical first step in this research was to examine current management doctrines that deal with benchmarking as a business imperative. Quality management, in its various forms, lies at the heart of business practice in many parts of the world and provides strong recognition of the role of benchmaiking. As Watson (1992, p. 19) puts it: "Once benchmarking becomes integrated into your continuous improvement process, you will have the opportunity 
to realise increased performance on a regular basis". Thus, the views of Deming, Juran, Crosby and other Quality Management pioneers, together with those of their successors, provide a broad context for considering the role of benchmarking in the achievement of best practice in OHS.

\section{- Organisational effectiveness}

It was mentioned earlier that organisational effectiveness is a critical lever in the achievement of excellent performance across a range of key result areas that have been defined for the enterprise, and that OHS performance is usually one such area. When properly executed, OHS programs should prove highly effective for an organisation (Tookey, 1987, p. 235; Oxenburgh, 1991, p. 14). Moreover, Toohey (1987, p. 237) asserts that workplace mishaps are a reflection of organisational effectiveness, while Winder (1995, p. 213) claims that performance in chemical safety is a reflection of the way an organisation functions. Several authors note the characteristics of organisations that are effective in their OHS efforts (Pardy, 1991, p. 16; Toohey, 1987, p. 238; Pope, 1992, p. 4; Pardy, 1991, p. 12) and terms such as "policy", "commitment", "measurement", "assignment of responsibilities" and "involvement" keep surfacing. Thus, the evidence from the literature is that organisational effectiveness and OHS performance appear to be closely linked.

\section{- Change management and management systems}

Change is an inevitable aspect of human life and successful organisations recognise that it needs to be managed. Changes thai might affect $\mathrm{OHS}$ include changes in staffing, proposals for new product, plant, processes or services, changes in work procedures, process modifications and software modifications (British Standards Institute, 1996, p. 13). Brandt (1997, p. $25)$ and Kotter (1999, p. 21) propose several elements that are associated with successful change initiatives. Lewis Lehr's comment (cited in Aune, 1991, p. 35) that Quality is the top priority and umbrella for managing change again shows the intertwining nature of the elements presently being considered. 
All enterprises have management systems to deal with inputs and outputs (Blewett \& Shaw, 1996c, p. 484) and many have developed formal systems for managing OHS (Redinger, 1997, p. 32). Again, there are strong links with quality management systems (Stephan, 2001, p. 247). The failure of management systems has been a consistent conclusion in many incident inquiry reports (Health and Safety Executive, 1992, p. 11).

\section{- Behavioural safety}

Enterprises that display excellence in their safety and health efforts usually have a behavioural safety component in their programs (Krause, 2000, $p$. 475). Some authors have pointed out the links between behaviour-based safety management, quality improvement and central organisational issues (Krause, Hidley \& Hodson, 1991, p. 50; Krause, 1997, p. 21). Behavioural processes become the means to continuously improve facilities, equipment, design and management systems (Krause, 2000, p. 478). The human element is widely recognised as a key factor in safety performance and is represented, along with management systems and the physical environment, as a component of the conceptual framework for this study (Figure 1).

\section{- Risk management}

Risk management consists of a set of well-defined steps that support better decision-making by providing greater insight into risks and their impacts (Standards Australia, 1999, p. iii). The steps shown in Figure 4 are an elaboration of those used for decades within the discipline of Industrial Hygiene, namely recognition, evaluation and control of workplace hazards (Harvey, 1980). Industrial Hygiene has a strong association with chemical safety and its techniques have been deployed extensively in this study. Today, risk management approaches are being applied well beyond OHS - to areas such as supply, information technology, engineering projects, maintenance, production, environmental management and community relations ( $P$. Janus, personal communication, December 13, 2002). Thus, risk management approaches are relevant to the efficient operation of organisations, as well as providing a framework to deal with workplace hazards. 
- Performance measurement, reporting and review

Performance measurement is widely regarded as a key management function or enabler. In the words of Strobach (1990, p. 42), "the heart of management control is measuring performance in quantitative, objective terms." In Paul Drucker's words (cited in Amis \& Booth, 1992, p. 45), "What gets measured gets done." Both leading and lagging indicators are available for use in OHS applications and it was thought appropriate to review what is being said in the literature before assessing what is being used within the facilities that have taken part in this study. Reporting systems vary from facility to facility, so this provided another variable to examine.

- Compliance, standards and auditing

Every organisation is covered by regulatory controls. Beiore any consideration can be applied to best practice elements, it is important to establish that the minimum standards expressed in regulations are being met. Hence, Booklet Three in the series deals with compliance. Regulations, standards, codes of practice and guidance notes were valuable sources of content for the booklets that were developed for the immediate study and will be useful for subsequent activities with various industry groups.

Auditing practices and procedures were dealt with in the Literature Review because it was likely that benchmarking approaches would bear some similarity to these. The processes and tools developed for this study were influenced by the literature and also by the researcher's experience in OHS auditing.

\section{- Benchmarking and technology transfer}

Clearly, a study on benchmarking requires a review of what has been done elsewhere, how it was carried out and what results or experiences were generated. Technology transfer, or more broadly, knowiedge transfer, is the logical outcome of a successful benchmarking exercise. 


\subsubsection{Design aspects}

A lot of attention was directed to the design of the booklets used for data gathering. This is consistent with Webster's observation that extreme care must be applied to make sure that the design and format aspects demonstrate professionalism, quality and attractiveness - as this will impact on the level of response to the material (Webster, 2000, p. 5). The challenge was to develop a product that would present in a simple, easyto-use format while applying a set of in-depth questions that would challenge the thought processes and elicit key information on what is being done differently, and well, on the site.

Earlier, mention was made of Chadwick, Bahr and Albrecht's outline of the strengths and weaknesses of qualitative inquiry (cited in Sarantakos, 1993 , p. 52). They note the time-consuming nature of this type of study and the potential for problems with objectivity and detachment. The study design sought to address the subjectivity issue by building in several measures to strengthen reliability and validity. These were described in the previous chapter.

\subsubsection{Research processes}

Giving the benchmarking partner a copy of the booklet materials and supplementary information well in advance of the first site visit made the interviews more productive and efficient, and made the whole exercise more transparent. This provided the host location with an opportunity to better understand its internal processes and initiatives in the area of interest, to prepare responses and to gather together supporting documentation in a timely way. In many respects, this paralleled the way that modern audits are conducted, wherein the recipient location is well aware of what subject areas will be covered, what questions will be asked and what further verification may be required by the auditor.

Equally important were:

- That the communications taking place in advance of the site visit were sufficient to ensure that the site didn't see the exercise as too daunting, too time consuming or of limited value. 
- That time spent on site was well structured and meaningful for all concerned.

- That the summary of findings and recommendations, per the sample format presented in Appendix 8, was developed and communicated to the host location in a timely way.

Initially, the approach was to use two of the booklets for the gathering of preliminary information. This seemed to work well. However, it soon became apparent that it was advantageous to have the facility, where possible, give some thought and early response to the content in Booklets 4-6. Data generated from this early effort was used to prepare for site visits and to make the time spent at the location more productive than it might otherwise have been.

The facility visit process followed the broad outline provided in Appendix 6 and went smoothly in all instances. Participating sites were very helpful with logistical support and were generous in the provision of resources to complete the exercise. In all cases, site management representatives were prepared to sign the informed consent form (Appendix 5) and thereby authorize use and publication of data generated in this study. Seven managers gave a broad approval - to cover use in any subsequent research that might arise (the first option on the consent form). The remaining manager indicated that his site's data should be applied to the present research only (the second option).

Facility representatives seemed to be open in their communications and willing to discuss both initiatives and opportunities for improvement at the site. Each facility visit included a walk-through survey element, as described by Harvey (1980). This enabled the researcher to use the senses of vision, hearing and smell to better understand the issues and challenges at the site and to verify some of the technical input received eariier from the OHS professional.

None of the walk-though exercises revealed situations at the site that were immediately dangerous to life or health. 


\subsubsection{Research tools}

A principal resouice for the study was the abundance of standards, regulations, codes of practice and other sources of direction or guidance that apply to an undertaking in Western Australia. These were utilised in the development of topics and sub-topics for the booklet series that was constructed to flush out "pockets of excellence" or potential best practice efforts. Best practice in a particular area can take many forms. It may be a product, service, tool, resource, system, concept or an approach. It may result from ideas that are genuinely original or may be a successful adaptation of something that has been implemented elsewhere. Examples of most of these emerged during the information-gathering phase of the work.

Booklets 4 - 6 provided the foundation for discussions that occurred onsite. Again, they provided a sound structure for the interviews and were generally well received.

The "Poor", "Fair", "Good" and "Excellent" ratings scale and attached criteria appeared to be easily understood and applied by facility representatives. A nomination of "Good" or "Excellent" led readily to questions from the researcher dealing with innovation and best practice at the site. The descriptor "Poor" had some limitations, in that to some degree it implies inadequate, or worse, negligent, performance. In some situations, the facility had not progressed down a particular path because it preferred instead to pursue other ways of achieving the same end result. Thus, the term "Poor" could be a harsh way of describing performance in some areas. The term "Not used" was more appropriate in some instances.

\subsubsection{Assumptions}

Eartier, reference was made to three assumptions that underpin the design of study processes and tools, namely that:

- The senior manager and the OHS professional at the location will be aware of innovation and exceptional practice that may be in evidence at the site. 
- Proceeding through the structured sequence of booklet questions will flush out pockets of excellence that may have potential to be used in a two-way exchange between benchmarking partners.

- Almost all sites have something to offer.

The first assumption was well tested at each facility visit. With mediumsized operations, the OHS professional was usually the only full-time OHS resource at the location and was thereby involved in ali significant safetyrelated activities. For larger facilities, there was normally a team of OHS professionals on-site and so study questionnaires were directed at the most senior professional, the person who had responsibilities that were plant-wide, rather than being limited to a particular function or departmental area. It is likely that such people would have carriage of any knowledge transfer with other facilities, so their ability to identify innovative efforts and to articulate what is different and attractive about such activity is an important element in setting up a successful exchange. For the facility manager, OHS is a key area of interest, but it is one of several functional areas to be addressed. Delegation is important, but the manager must set direction, apply resources and monitor performance against objectives. In the course of the present study the managers were usually able to demonstrate a broad knowledge of the functional area of OHS, the principal systems and tools being applied on-site and how well the site was performing against plan.

As mentioned earlier, facility representatives were supportive of the process and the use of the booklet series to work through important OHS elements and to identify pockets of excellence in a systematic way. One participant commented with some surprise that, for him, the answers were revealing in that they highlighted how his site was strong on people-related controls, such as rules, procedures, protective equipment and similar, but relatively weak in aspects that have higher standing in the hierarchy of controls.

With regard to the third assumption, all sites nominated items that they considered to be critical success factors for chemical safety. Without 
exception, these were areas that they believed were being actively pursued at the facility. Booklets Four, Five and Six contain 45 ratings questions, where a "Good" or "Excellent" response leads to identification of potential benchmarking opportunities. The number of Good or Excellent responses varied from thirteen at Facility Seven to forty at Facility Two. Thus, all sites believed that they had some approaches or innovations that compared well with what was available elsewhere.

\subsection{Intra-site observations and comparisons}

\subsubsection{Facility One}

As indicated earlier, Facility One was a large alumina refinery that processes bauxite from the Darling Ranges. The Bayer Process has a wide range of attendant physical, chemical and ergonomic hazards, and the facility responds to this with strong OHS programs. These are adequately led, with a high level of management visibility, and are resourced with a substantial contingent of OHS professionals. The facility seeks to engage the workforce in its safety initiatives and has structures in support of this. Overall, the approach to occupational health and safety at the site is consistent with that which is advocated in the safety literature and which has been described in some depth in Chapter Two.

There are effective systems in place with regard to ordering, vetting, supply and distribution of hazardous materials. Chemical safety systems at the site are strong. There is a strong emphasis on engineering controls, safe systems of work, procedures, training and communication.

Facility One appears to have a high level of compliance with the chemical safety provisions of the Mines Safoty and Inspection Regulations 1995. The facility has in place an inventory of materials on site, a register of MSDSs is maintained and is available to the workforce, personal protective equipment is appropriately managed, the hierarchy of controls appears to be well understood on site, and regulatory requirements appear to be met, and exceeded, in regard to the conduct of risk assessments, labelling, first aid, health surveillance and monitoring of workroom air. There are 
adequate arrangements in place for archiving of MSDSs relating to superseded materials or earfier formulations. However, there were several opportunities for improvement. These were centred on practices associated with decanting and further use of chemical substances, secondary labelling of containers and control of all atmospheric contaminants to levels below those specified in the current Worksafe Australia Exposure Standards publication.

The facility scored well with regard to the management enablers of leadership, commitment, planning, organisation, training, communication and measurement. Management visibility at the site is enhanced by initiatives such as the management safety contact program, described earlier. OHS targets are set, funds are allocated and progress is monitored. The site has excellent systems for tracking and reporting OHS performance, with several benchmarking opportunities in this area. The strength of the management systems at the site has been verified via the Department of Industry and Resources' audit program and the relatively high score $(89.7 \%)$ that was allocated to the location. Opportunities for improvement exist in relation to formal evaluation of the effectiveness of training, in some aspects of the provision of refresher training and in making better use of extemal resources to support the OHS effort.

Technical initiatives at the site cover atmospheric monitoring programs, formalised workplace inspections, job safety analyses and personal protective equipment controls. There is a highly developed Industrial Hygiene program in evidence at the location and all of the program elements mentioned in Booklet 5 appear to be in place. Exposure baselines have been established, job dictionaries are in use and chemical inventories are applied in training, awareness-building and auditing applications. Qualitative and quantitative exposure assessments are carried out with the support of a purpose-built, corporate software package. Formal processes are applied to feasibility assessments and to subsequent engineering, procurement, work practice or personal protective equipment controls. 
With regard to the people aspect of OHS, the site is relatively new to the behavioural safety area and, in particular, the element that deais with task observations. However, substantial progress has been made o rer the last 18 months. In 2002, Operating Centres at the location carried out a combined total of 20,000 task observations and management contact initiatives. There is strong engagement of the workforce in promotional efforts. Additionally, motivation and recognition efforts are well deployed at the site. Attitude and morale surveys have been used sporadically in the past to gauge likely employee support or reaction to proposals for change.

\subsubsection{Facility Two}

As described earlier, Facility Two was a mid-sized bauxite mining operation located in the Darling Ranges, to the south of Perth. It belongs to the same organisation as Facility One. As such, it would be expected to use similar tools and processes in dealing with OHS. While this is so, the site did demonstrate several locally developed innovations and had a culture and certain practices built around its location, smaller size (compared to an alumina refinery) and type of operation.

The management of hazardous materials at the location is given effect by systems that cover ordering, vetting, supply and distribution aspects. Overall, the OHS effort at the site is significant. There is emphasis on leadership, target-setting, training, employee involvement and performance meásurement, reporting and review.

There was a high level of compliance with the chemical safety provisions of the Mines Safety and Inspection Regulations 1995. The facility has in place an inventory of materials held on site, a register of MSDSs is maintained and is available to the workforce, personal protective equipment is appropriately managed, the hierarchy of controls appears to be well understood on site, and regulatory requirements appear to be met in regard to the conduct of risk assessments, labelling, first aid, health surveillance and assessment of workroom air quality. There appeared to be several opportunities for improvement. These were centred on maintenance of non-disposable respirators, practices associated with 
decanting and further use of chemical substances, secondary labelling after transfer of hazardous substances to smaller, on-site containers and control of all atmospheric contaminants to levels below those specified in the current Worksafe Australia Exposure Standards publication.

The mine scored well with regard to the management enablers of leadership, commitment, planning, organisation, training, communication and measurement. There is good management visibility, OHS targets are set, funds are allocated and progress is monitored. The strength of the management systems at the site has been confirmed via the Department of Industry and Resources' audit program and the above average score $(86 \%)$ that was allocated to the location. Opportunities for improvement exist in relation to formal evaluation of the effectiveness of training, access to, and utilisation of, internal resources, and in the general area of behaviour, including compliance with rules and procedures.

Technical efforts at the site include deployment of monitoring programs for a limited number of air contaminants, formalised workplace inspections, job safety analyses and personal protective equipment controls. There is a well-developed Industrial Hygiene program in evidence at the location, using professional resources from the refinery. All of the program elements mentioned in Booklet 5 appear to be in place. Exposure baselines have been established, job dictionaries are in use and chemical inventories are in place and used in training, awareness-building and auditing applications. Qualitative and quantitative exposure assessments are carried out with the support of a purpose-built, corporate software package. Formal processes are applied to feasibility assessments and to subsequent engineering, procurement, work practice or personal protective equipment controls.

In the people area, the site engages in a behavioural safety program via its MATE program. The latter includes a "golf card" innovation to assist task observation efforts. There is a weekly, formalised management contact program and such activity is measured and reviewed. Additionally, there is strong engagement of the workforce in team recognition events. Attitude 
and morale surveys have been used sporadically in the past to gauge likely employee support or reaction to proposals for change, so this represents a potential improvement opportunity.

\subsubsection{Facility Three}

Facility Three was different to the other locations represented in the study in that it is a chemicals manufacturing operation, with tank terminal and transport services. It is a major supplier of chemicals to the mining industry in Western Australia and this linkage made it an attractive candidate for inclusion in the study.

There are effective systems in place with regard to ordering, vetting, supply and distribution of hazardous materials. Site programs seek to integrate environmental, safety, health and quality aspects of the operations. There is emphasis at the facility on implementation of the organisation's risk management system, compliance auditing, OHS and first-aid training, effective communication and emergency response capability. The location has well established policies, procedures and reporting protocols.

There is a strong commitment at the site to ensuring that statutory requirements for dangerous goods are being met. As mentioned earlier, the site is audited every 12 months against the 21 elements of its risk management system. The mean sarre from the most recent (2002) audit was $92 \%$. The location is under the jurisdiction of Worksafe Western Australia for OHS matters, while being under the control of the Department of Industry and Resources of Western Australia for matters relating to dangerous goods. The facility has in place an inventory of materials held on site, a register of MSDSs is maintained and is available to the workforce, personal proiective equipment is appropriately managed, the hierarchy of controls appears to be reasonably well understood on site, and regulatory requirements appear to be met in regard to the conduct of risk assessments, labelling, first aid, health surveillance and monitoring of workroom air. There were a few opportunities for improvement. These were in areas such as the archiving of MSDSs relating to superseded materials or earlier formulations and control of all atmospheric 
contaminants to levels below thove specified in the current Worksafe Australia Exposure Standards publication.

In the "management of work" element, the facility demonstrated its strengths with regard to the management enablers of leadership, commitment, planning, organisation, training, communication and measurement. There is good management visibility, OHS targets are set, funds are allocated and progress is monitored. Opportunities for improvement exist in relation to target-setting, integration of contractor plans with those of the facility, utilisation of internal resources, use of leading or positive performance indicators, and in the area of communication and deployment of performance measures. The site representative identiffed training as a critical success factor. However, refresher training, safety representative training and formal evaluation of the effectiveness of training were all self-rated as "Fair".

Its workplace environment initiatives cover atmospheric monitoring programs, formalised workplace inspections and qualitative assessments, together with engineering, work practice and personal protective equipment controls. Its air sampling programs would be stronger if job dictionaries, sampling plans and exposure baseline were put in place, and if statistical treatments were applied.

In the people area, the site engages in various promotional efforts with OHS and there is a recognition system that is triggered when key safety performance milestones are achieved. There is no formal behavioural safety or peer-based task observation program in place and this may be another opportunity for improvement. The organisation might benefit by promoting and supporting the role of elected safety representatives, via additional training, recognition and clear expression of roles, responsibilities and performance expectations. Attitude surveys and morale surveys have been used with success elsewhere (Lindsay, Manning \& Petrick, 1991, p. 100) and these provide a mechanism for identifying barriers to good safety performance, as perceived by the workforce, and for gauging likely employee support for $\mathrm{OHS}$ initiatives. 


\subsubsection{Facility Four}

Facility Four was a large contract gold mining organisation operating on behalf of a client at a site in the Murchison district of Western Australia. The client company has both above- and below-ground mining services being carried out by contractors, while it pursues gold extraction with its own resources (see Facility Five description).

The facility manages its hazardous materials stocks via systems that deal with ordering, vetting, supply and distribution aspects. Safety has a high profile in the organisation. There is emphasis at the site on deployment of OHS rules and procedures, first-aid training and emergency response capability.

Certain sections of the Mines Safety and Inspection Regulations 1995 desrribe the minimum requirements with respect to chemical safety on mine sites. The facility addresses these requirements with a list of materials used on site, a register of MSDSs that is maintained and made available to the workforce, and personal protective equipment that is appropriately managed. In addition, the hierarchy of controls appears to be reasonably well understood, and regulatory requirements appear to be met in regard to the conduct of risk assessments, labelling, first aid, health surveillance and monitoring of workroom air. There were several opportunities for improvement. These were centred on strengthening the chemical inventory arrangements, archiving of MSDSs relating to superseded materials or earlier formulations, secondary labelling of containers, documentation of formal risk assessments, training employees in the use of an MSDS and systems of work attached to use of hazardous materials in enclosed spaces.

The site was able to demonstrate a set of initiatives in the organisation of work area, notably with the management enablers of leadership, commitment, planning, organisation, training, communication and measurement. There is good management presence around the site, OHS targets are set, funds are allocated and progress is monitored. The Company has not been subject to a management systems audit of the kind 
conducted by the Department of Industry and Resources' audit program and described earlier. Auditing attention tends to be directed to the occupier's operations rather than to the contractor who provides a service to the client. For Facility Four, opportunities for improvement exist in relation to formal evaluation of the effectiveness of training, linkage of OHS plans with those of the client and with sub-contractors, increasing the level of employee participation and ownership of safety initiatives, and in the area of communication and deployment of performance measures.

The facility deploys formalised workplace inspections, job safety analyses and personal protective equipment controls. Its air sampling programs are limited and would be stronger if an exposure baseline was put in place. In addition, programs would be improved if statistical treatments were applied and certain additional measures were taken in relation to communication of results.

In the people area, the contractor engages in a strong, supervisor-led task observation program. This is supported by comprehensive guidance material. Safety programs at the site appear to have a strong top-down character and outcomes are likely to improve if employee involvement and ownership levels can be improved. There has been no use of attitude or morale surveys as a mechanism for identifying barriers to good safety performance, as perceived by the workforce, and for gauging likely employee support for OHS initiatives. Similarly, there is no behavioural safety or peer-based task observation program in place and this may be another opportunity for improvement.

\subsubsection{Facility Five}

Facility Five was a medium-sized gold mining, milling and extraction operation in the Murchison district of Western Australia. The Company has contractors engaged to carry out both above- and below-ground mining operations on its behalf, while it operates a Carbon-in-Leach plant and carries out the gold extraction element itself. 
Suitable measures are in place with regard to ordering, vetting, supply and distribution of hazardous materials, and broader management systems for OHS are strong. There is emphasis on induction training, accident investigation, reporting protocois and emergency response capability at the site.

There appears to be satisfactory compliance with the chemical safety provisions of the Mines Safety and inspection Regulations 1995. The facility has in place an inventory of materials held on site, a register of MSDSs is maintained and is available to the workforce, personal protective equipment is appropriately managed, the hierarchy of controls appears to be reasonably well understood on site, and regulatory requirements appear to be met in regard to the conduct of risk assessments, labelling, first aid, health surveillance and monitoring of workroom air. There were several opportunities for improvement. These were associated with archiving of MSDSs relating to superseded materials or earlier formulations, secondary labelling of containers after transfer of hazardous substances to smaller, on-site containers, documentation of formal risk assessments, training employees in the use of an MSDS and systems of work attached to use of hazardous materials in enclosed spaces.

The facility was active with the management enablers of leadership, commitment, planning, organisation, training, communication and measurement. There is good management visibility, OHS targets are set, funds are allocated and progress is monitored. The Department of Industry and Resources has endorsed the strength of the management systems at the site through its audit program. A relatively high score of $94 \%$ was obtained by the location. Opportunities for improvement exist in relation to formal evaluation of the effectiveness of training, increasing the level of employee participation and ownership of safety initiatives, and in the area of communication and deployment of additional leading indicators of performance.

Technical initiatives at the site include atmospheric monitoring programs, although these do not extend very far beyond the statutory requirements 
expressed in the CONTAM system. There is emphasis on formalised workplace inspections, job safety analyses and personal protective equipment controls. Air sampling programs would be stronger if an exposure baseline was put in place, if statistical treatments were applied and certain additional measures were taken in relation to communication of results. There is a need for the site to do more with regard to investigating exposure to tailings dust, controlling the work practices that make exposure to this dust possible and communication of results and counter-measures.

In the people area, the location undertakes a variety of promotional efforts in the OHS area and there is a recognition system that is activated when key safety performance milestones are reached. Safety programs at the site appear to have a strong top-down character and outcomes are likely to improve if employee involvement and ownership levels can be improved. There is no behavioural safety or peer-based task observation program in place at the site and this may be another opportunity for improvement. The organisation might benefit by promoting and supporting the role of elected safety representatives, via additional training, recognition and clear expression of roles, responsibilities and performance expectations. As mentioned earlier, attitude surveys and morale surveys have been used with success elsewhere (Lindsay, Manning \& Petrick, 1991, p. 100) and these provide a mechanism for identifying barriers to good safety performance, as perceived by the workforce, and for gauging likely employee support for OHS initiatives.

\subsubsection{Facility Six}

Facility Six was an open-cut, iron ore mine in the Pilbara Region of Western Australia. It is linked to several, smaller satellite ore bodies. The site has a solid complement of safety, health/hygiene, fire, security and emergency services personnel at its disposal.

There are effective systems in use with regard to ordering, vetting, supply and distribution of hazardous materials. Overall, OHS systems are well developed. There is emphasis at the location on risk assessments, audits, 
behavioural safety and structures to deliver induction, refresher and Safety Representative training.

There is a high level of compliance with the chemical safety provisions of the Mines Safety and Inspection Regulations 1995. The facility has in place an inventory of materials on site, a register of MSDSs is maintained and is available to the workforce, personal protective equipment is appropriately managed, the hierarchy of controls appears to be reasonably well understood, and regulatory requirements appear to be met in regard to the conduct of risk assessments, labelling, first aid, health surveillance and monitoring of workroom air. One of the items on the inspection checklist addresses an issue that is a weakness for many facilities, namely correct labelling of containers that hold decanted materials. There were several opportunities for improvement. These were centred on compliance to rules and procedures, and ensuring control of all atmospheric contaminants to levels below those specified in the current Worksafe Australia Exposure Standards publication.

The facility scored well with regard to the management enablers of leadership, commitment, planning, organisation, training, communication and measurement. There is excelient management visibility and policy enunciation, OHS targets are set, funds are allocated and progress is monitored. The strength of the management systems at the site has been verified via the Department of Industry and Resources' audit program and the relatively high score $(89 \%)$ that was assigned to the location. Opportunities for improvement exist in relation to formal evaluation of the effectiveness of training, strategic planning, use of leading indicators of performance and in making better use of external resources to support the OHS effort.

The Site Industrial Hygienist uses walk-though survey techniques to check departmental inventories of chemicals and to evaluate the measures being taken in regard to storage, handling, application of controls, and similar. Although job dictionaries are not used, the workforce is stratified into groups with similar exposures. Exposure baselines have been established 
and statistical treatments are applied to measurement results. Feasibility (for control) assessments are not applied in a consistent way. However, procurement controls, work practice controls and personal protective equipment controls are well developed.

In the people area, the facility has made progress with its behavioural safety efforts. This is delivered via the wider organisation's Safe Act Observation (SAO) Program. This initiative involves both management and the workforce, and is supported by well-defined processes and materials. Safety promotion, compliance with rules, including the wearing of personal protective equipment, and management contacts on OHS are all components of the SAO Program. Motivation and recognition efforts, together with attitude and morale surveys are delivered in sporadic fashion, so this may be an opportunity for improvement.

\subsubsection{Facility Seven}

Facility Seven was a gold mining, milling and extraction operation located to the south of Kalgoorlie. The complex consists of three open cut pits, one underground operation and two gold processing plants. The operation is owned by the same organisation that is responsible for Facility Five, described earlier. Again, the above-ground and below ground mining components are carried out by contractors that specialise in this work.

The facility manages its hazardous materials stocks via systems that deal with ordering, vetting, supply and distribution aspects. The Loss Control Manager plays a central role in this. There is emphasis at the site on deployment of the broader organisation's "Whole of Mining Risk Assessment" protocols, together with OHS rules and procedures, first-aid training, accident investigation processes and emergency response capability.

Certain sections of the Mines Safety and Inspection Regulations 1995 describe the minimum requirements with respect to chemical safety on mine sites. The facility addresses these requirements with a list of materials used on site, a register of MSDSs that is maintained and made 
accessible to the workforce, and personal protective equipment that is made available where needed. In addition, the hierarchy of controls appears to be reasonably well understood, at least at a management level, and regulatory requirements appear to be met in regard to the conduct of risk assessments, labelling, first aid, health surveillance and monitoring of workroom air. There were several opportunities for improvement. These were centred on strengthening the chemical inventory arrangements, archiving of MSDSs relating to superseded materials or earlier formulations, secondary labelling of containers and documentation of formal risk assessments and duties that may give rise to exposure.

The site was active in the organisation of work area, notably with the management enablers of strategic planning, training (at induction and with that provided to Safety Representatives), access to external resources, hazard communication and use of lagging indicators. The facility has not been subject to a management systems audit of the kind conducted by the Department of Industry and Resources' audit program and described eariier. For Facility Seven, opportunities for improvement exist in relation to the development and application of leading indicators of performance, target setting, application of resources, formal evaluation of the effectiveness of training, linkage of OHS plans with those of the contracting organisations, and in increasing the level of employee participation and ownership of safety initiztives.

The facility deploys formalised workplace inspections, job safety analyses, procurement controls for chemicals and personal protective equipment controls. Its air sampling programs are limited and would be stronger if an exposure baseline was put in place. In addition, programs would be improved if statistical treatments were applied and certain additional measures were taken in relation to communication of results.

There are several improvement opportunities available in the people area. Safety programs at the site appear to have a strong top-down character and outcomes are likely to improve if employee involvement and ownership levels can be improved. There is no substantive, formalised 
motivation/recognition program to encourage participation in OHS, nor has use been made of attitude or morale surveys as a mechanism for identifying barriers to good safety performance, as perceived by the workforce, and for gauging likely employee support for OHS initiatives. Similarly, there is no behavioural safety or peer-based task observation program in place and this may be another opportunity for improvement.

\subsubsection{Facility Eight}

Facility Eight was a nickel refinery located in the Kwinana industrial zone. it produces nickel briquettes and nickel powder.

There are effective systems in place with regard to ordering, vetting, supply and distribution of hazardous materials. The Environmental Coordinator plays a key role in this activity. Overall, the facility and the broader organisation to which it belongs are strong in the business planning area and several initiatives spring from this. There is emphasis at the site on inventory control, storage and handling arrangements and procedures, first-aid training and emergency response.

Certain sections of the Mines Safety and Inspection Regulations 1995 set out the minimum requirements with regard to chemical safety on mine sites and mineral processing piants. The facility has in place an inventory of materials on site, a register of MSDSs is maintained and is available to the workforce, personal protective equipment is approprlately managed, the hierarchy of controls appears to be reasonably well understood on site, and regulatory requirements appear to be met in regard to the conduct of risk assessments, procedures for working in enclosed spaces, disposal of containers, labelling, first aid, health surveillance and monitoring of workroom air. There were several opportunities for improvement. These were centred on archiving of MSDSs relating to superseded materials or earlier formulations, secondary labelling of containers, documentation of formal risk assessments and duties that might give rise to exposure, training employees in the use of an MSDS and record-keeping in relation to training in chemical safety. 
The facility scored well with regard to the management enablers of leadership, commitment, planning and organisation, but less well in the areas of training, communication and measurement. There is good management visibility, management of change procedures are well developed, OHS targets are set, funds are allocated and progress is monitored. The strength of the management systems at the site has been verified via the Department of Industry and Resources' audit program and the relatively high score that was allocated to the location $(94 \%)$. Opportunities for improvement exist in relation to deployment of management systems, roll out of training, particularly as it relates to chemical safety, formal evaluation of the effectiveness of training, increasing the level of employee participation and ownership of safety initiatives and in the area of communication and deployment of performance measures. In regard to the latter, it would be useful to establish measures that could be applied to the elements identified by the site as critical success factors for chemical safety.

The facility has well established policies, procedures and reporting protocols. Its technical initiatives cover atmospheric monitoring programs, the development of a more extensive exposure database, formalised workplace inspections, job safety analyses, procedural controls, particularly in relation to work in enclosed spaces, and personal protective equipment controls. Its air sampling programs would be stronger if statistical treatments were applied and certain additional measures were taken in relation to communication of results.

In the people area, the site engages in various promotional efforts with safety and has surveyed its workforce to determine attitudes to safety and its delivery. Safety programs at the site appear to have a strong top-down character and outcomes are likely to improve if employee involvement and ownership levels can be improved. There is no behavioural safety or task observation program in place, nor is thore any substantive, formalised motivation/recognition program to encourage participation in OHS. These may be other opportunities for improvement. 


\subsection{Inter-site observations and comparisons}

\subsubsection{Diversity}

There was considerable diversity in the characteristics of the sites participating in this study. They varied from small, relatively simple mining operations to larger, more complex processing operations, with a wider range of attendant OHS risks. Some facilities were engaged in below ground mining operations while others were surface operations. One site was a principal supplier of chemicals to the mining industry in Western Australia, while another "facility" was a contract-mining operation, providing an ore extraction service to a client mining company. Some sites were close to Perth, while others were in remote locations, hundreds of kilometres from the capital city. Some sites had a very stable, local workforce while others had a high turnover labour situation, with people operating on a "fly-in/fly-out" basis.

Diversity of this kind was considered to be an advantage in that study processes and tools would be put to the test in situations that reflect some of the key variables that prevail in Western Australian Industry.

\subsubsection{Booklet One materials}

Booklet One was designed to gather some initial facts about the facility its function, contact details, circumstances relating to the deployment of its workforce, together with OHS challenges and arrangements for addressing these.

\section{- Organisation for OHS}

Smaller facilities usually had the bulk of OHS respc sibilities vested in one individual, with that person reporting directly to the site manager. On the other hand, larger sites typically employed a team of OHS professionals, with some people having specialist backgrounds. Thus, they usually had access to a broader and deeper knowledge base. The usage of elected Safety Representatives was quite vartable. Where the management style at the facility was of a top-down nature, there was little emphasis on 
workforce involvement and so Safety Representatives were either not used or not valued.

\section{- Principal OHS issues and challenges}

The mining and mineral processing facilities included in this study share many common OHS issues and challenges. Operation and maintenance of moblie equipment and fixed plant would be an example of this. Physical agents such as noise, vibration, radiation and thermal stress are commonly encountered. In the area of chemical agents, dust (from ore and waste sources), acids, alkalis, compressed gases, fuels, lubricants, cleaning agents, sprays, adhesives, welding gases and fumes, and workshop solvents and other chernicals are in clear evidence.

- Other relevant information

All sites were part of a wider organisation and were able to draw on knowledge and experience from elsewhere. $X x$ of the study sites had been subject to a Department of Industry and Resources audit of management systems at some point during the past five years. Audit scores indicated that the locations were well placed compared to their peer group within the mining and mineral processing industry. In all cases, their scores were above the mean for the Industry. Similarly, Lost Time Injury Frequency Rates for $\mathbf{2 0 0 2}$ ranged from zero to 6.3 per million hours worked. This demonstrates a safety performance that is, with two exceptions, better than the mean figure of 4.3 per million hours worked for metalliferous component of the Western Australian Mining industry, as published by the Department of Industry and Resources (2003, p. 14). This would suggest that organisations that show interest in benchmarking activity are likely to be superior performers in OHS and to believe that they have something to offer in a benchmarking exchange.

\subsubsection{Booklet Two materials}

Bocklet Two was part of the initial package of materials sent to the participating location and was designed to elicit preliminary information on OHS, in general, and chemical safety, in particular. 
- The Yes/No questionnaire (general OHS)

All of the locations responded in the affirmative to the questions posed in Part A1 of Booklet Two. Since the nine "yes/no" questions relate to the characteristics of organisational effectiveness in OHS, as described by Toohey (1987, p. 238), then the foundation is there for a strong program and there should be some benchmarking potential within each site.

- Benchmarking, best practice, and technology transfer

All participating facilities indicated that previous efforts with respect to benchmarking in OHS had been of a very limited and informai nature. This provided support for the premise that this study is novel and has the potential to pioneer a more formal and effective approach to benchmarking between like-minded organisations in Western Australia.

- Measurement - Lagging indicators

The most widely used lagging indicator within the participating sites was Lost Time Injury Frequency Rate (LTIFR). This measure has a number of limitations, as described in Chapter Two. Nevertheless, it is well understood, widely accepted and routinely reported to Government agencies and other stakeholders. Thus, it was a data element that was readily available and was a useful item for inter-site comparisons.

- Measurement - leading indicators

All facilities had some measures that fell under this heading. Those with established behavioural safety programs utilised activity-based measures such as the number of task observations or the number of management safety contacts carried out during the month. Other popular activity-based indicators were the number of training sessions held, the number of hazards identified and logged, the number of safety meetings held and similar. Some sites opted for measures that are more outcome oriented such as the number of corrective actions carried out, self-audit scores, compliance audit results and similar. The purpose of the question was to explore the extent of use of leading indicators. It was thought that facilities that made effective use of such measures would be likely to be innovative in other areas. This was generally the case. While the wide range of 
indicators in use worked against cross-site comparisons of scores, performance measurement is a key management enabler and so this subject represents an attractive area for future benchmarking activity

\section{- Self-ranking in OHS}

With one exception, the facilities that took part in the study ranked their OHS performance and strategies in the top quartile relative to the local Mining Industry. This seems somewhat unrealistic given the range of facilities encompassed by the study. In part, it could be explained in the sense that all sites taking part were volunteers that welcomed the opportunity to participate and felt they had something to offer. In other words, a certain amount of self-selection may have occurred, with the project having appeal to better-performing, more confident organisations. However, some program elements that the researcher encountered appeared to be quite rudimentary, while being considered to be "Good" or "Excellent" by the facility representative. This may reflect human nature and the desire to be seen to excel or it may reflect a lack of knowledge of what constitutes excellence for a particular area of activity. The latter explanation fits with the observation by Wiarda and Luria (1997, p. 2), alluded to earlier, who have pointed out that benchmarking is invaluable for its shock value and that managers sometimes act with disbelief at gaps that may exist between performance at their site and practice at other sites. According to the same authors, facilities almost universally overrate their performance. They found, in a survey of manufacturing plants, that $81 \%$ of respondents thought that they ranked in the top quartile of their industry (Wiarda \& Luria, 1997, p. 1). Activities that may be seen as inhouse strengths may be modest in relation to comparable activities at other sites. Such is the value of benchmarking.

\section{- Potential benchmarking partners}

Facility representatives nominated a wide variety of organisations as "most admired" or potential benchmarking partners. Some of these were high profile international organisations without a manufacturing presence in Western Australia, such as DuPont and Dow Chemicals. Some were companies that operate locally and enjoy a strong reputation for OHS, 
st:ch as Woodside Petroleum and Alcoa of Australia. A few interviewees saw the MINEX award system, with its peer scrutiny features, as a ready source of potential benchmarking partners. Other nominations were more obscure, with operations outside the chemical manufacturing and mineral processing areas, such as the South A.frican Pulp and Paper Industry.

\section{- The Yes/No questionnaire (chemical safety)}

Locations responded in the affirmative to most of the questions posed in Part B1 of Booklet Two. Since the eleven "yes/no" questions relate to the essentials of chemical safety, as described by Chesson (1990, p. 406) and the Department of Minerals and Energy (1995a, 1997a), then the foundation is there for a solidly-based program and there should be some benchmarking potential within each facility.

\section{- Critical success factors}

Site representatives were asked to nominate four critical success factors in relation to chemical safety at their location. Responses were varied, although core competencies such as leadership, organisation, training and commitment were prominent in the nominations, together with vetting arrangements, maintaining an up-to-date chemical inventory and access to chemical information via commercial systems such as Chem Alert. The latter clearly has good market penetration in Western Australia.

\section{- Performance measurement}

All facilities used traditional lagging indicators, such as LTIFR, to track OHS performance. Leading indicators were less well entrenched as a tool for monitoring performance. However, some sites use activity measures to good effect. These include the number of training sessions delivered, the number of task observations carried out, the number of management safety contacts made, and similar. Remarkably though, few sites had performance measures that corresponded to the items nominated as critical succuss factors for chemical safety.

\section{- Innovations}

As indicated earlier, innovations can take many forms. They may be related to a product, service, tool, resource, system, concept or an 
approach. They may result from ideas that are genuinely original or may be a successful adaptation of something that has been implemented elsewhere. Examples of each of these were seen during the exercise.

\subsubsection{Booklet Three materials}

Booklet Three was used to assess the level of compliance with Part 7, Division 3 of the Mines Safety and inspection Regulations 1995. The regulations address topics such as MSDSs, container design and disposal, labelling, registers, enclosed spaces, risk assessment, risk reduction, atmospheric monitoring, health surveillance, first aid and miscellaneous matters attached to training.

Compliance levels were generally quite high. All sites made use of commercially available systems, such as Chem Alert, to support their obligations with regard to suitable MSDSs, training and communication of hazardous materials information.

There were a few areas that stood out as offering opportunities for improvement. These were in relation to site arrangements for transfer of materials into smaller containers, application of secondary labelling and disposal of used containers.

\subsubsection{Booklet Four materials}

Booklet Four was designed to identify innovation and best practice associated with key management enablers for OHS. These are leadership, commitment, planning, organisation, training, communication and measurement, all of which are well recognised in the literature described earlier. All facilities that took part in the study had strong management systems. This became apparent during the interview process and subsequent verification activities. Further confirmation came from the scores obtained from an independent audit of management systems, as performed by officers of the Department of Industry and Resources (formerly the Department of Minerals and Petroleum Resources of Western Australia). 
- Leadership

As Juran (1991, p. 7) observed, leadership is a key to getting results in OHS. All sites participating in this study were able to demonstrate the importance they attached to visible and active leadership. Some initiatives were formalised, tracked and used in performance appraisals. The management safety contact effort, as deployed by Facilities One and Two, was an example of this. But leadership can exist at many levels. Some sites empowered their employees to the extent that elected Safety and Health Representatives were leading much of the safety effort within their natural work groups.

\section{- Commitment}

Most interviewees saw commitment as being expressed in terms of the allocation of people and equipment resources to address the issue at hand, together with making provision in the location or departmentat budget to fund important $\mathrm{OHS}$ projects.

\section{- Planning}

There was evidence at all sites that OHS was integrated with broader organisational plans. Targets are set for both short-term and long-term activities and progress is monitored. Contractors play a significant production or maintenance role at most of the facilities. Their OHS plans were often reviewed as part of pre-qualification checks. However, there was some variability in regard to the extent to which their plans are integrated with those of the site.

\section{- Organisation}

Key requirements in this area are that OHS rescurces are sufficient, that the people involved have ready access to senior site management and that the organisational structure encourages interaction of OHS professionals with line management and shop floor personnel. This was generally the case at the study locations.

\section{- Training}

The MARCSTA induction package is now an entrenched and significant part of OHS training within the Western Australian Mining Industry ( $P$. 
Gilroy, personal communication, March 3, 2001). Study sites used the one-day MARCSTA course to cover general OHS subjects before delivering more specific, site-related training. Most sites had a safety representative system and elected individuals received the five-day introductory training course, per statutory requirements. Follow-up training, specifically aimed at safety representatives, was limited for most sites. All facilities provided refresher-style training, with delivery via safety meetings, safety summits, off-site workshops, tool-box sessions, selfpaced CD-ROM sessions, and similar.

\section{- Communication}

Hazard communication initiatives at the study sites took many forms. There were safety alert systems, feedback from air sampling exercises, use of database information on hazardous materials, awareness training, safety meetings and similar. Safety targets were also conveyed in a variety of ways and feedback on performance was channelled through toolbox meetings, electronic and hard copy reports, notices, newsietters and other presentations.

\section{- Measurement}

Lagging indicators, such as Lost Time Injury Frequency Rates (LTIFR), Medical Treatment Injuries (MTI) and Minor (first-aid) Injuries (MI), were popular at participating sites. There are statutory obligations attached to reporting of the more serious forms of injury to the regulatory agency. Leading indicators of performance were not as well developed, but there was evidence of initiatives such as the tracking of the number of safety meetings held, the number of training sessions held, the number of inspections or audits carried out, and similar.

\subsubsection{Booklet Five materials}

Booklet Five is centred on technical elements within a risk management framework, namely hazard identification, risk assessment and risk control. It also contains a section that may be used to assess the strength of the Industrial Hygiene program at the location. 


\section{- Hazard Identification}

Hazard recognition approaches were quite varied. Some sites nominated their hazard log reporting systems and workplace inspection programs as central to their efforts under this heading. Others saw the MSDS as a key source of information on hazards associated with site. A few facilities used departmental inventories of chemicals and checklists as the basis for training, awareness-building and auditing applications.

\section{- Risk Assessment}

All of the sites engaged in formal risk assessment activities to some degree. All sought to meet regulatory requirements in terms of monitoring for atmospheric contaminants, that is, they were set up to meet the State Government's CONTAM requirements as a minimum. A few went beyond this and used sophisticated software to develop exposure baselines, sampling schedules and to apply statistical treatment to results.

\section{- Risk Control}

Most sites were strong in the procedural area and in terms of use of personal protective equipment, but weaker in their pursuit of substitution/elimination alternatives and other elements that reside in the upper part of the hierarchy of controls.

\section{- Industrial Hygiene Program Development}

For most sites, Industrial Hygiene programs were quite limited. Where the discipline was well established with in-house professional resources in support (Facilities One, Two and Six), programs were robust, comprehensive and well conducted. Only the largest of the participating sites employed Industrial Hygienists, although smaller sites had some access to such professionals through contract services.

\subsubsection{Booklet Six materials}

Booklet Six was designed to seek out exceptional practice or innovations with respect to people factors. Ratings questions were framed around eight sub-topics. 
Three facilities had behavioural safety programs that were reasonably well developed, while another site was at an early stage in instituting such a program.

- Motivation and Recognition Initiatives

Motivation and recognition initiatives were prominent at sites that placed high value on employee involvement in $\mathrm{OHS}$ and had structures in place to facilitate this.

\section{- Attitude and Morale Surveys}

These tended to be deployed in sporadic fashion. There were no examples of such surveys being used in a routine way to shape the facility's approach on OHS issues or to guide decision-making. Lindsay, Manning and Petrick (1991, p. 106) have argued persulasively that morale is linked to safe behaviour and to the financial performance of the organisation. Thus, systematic deployment of such tools represents an area of opportunity for most sites.

\section{- Safety (Management) Contact Programs}

Formal programs of the kind used by the DuPont organisation were limited to Facilities One and Two. Here, expectations (of the manager and supervisor) were established and contact activity was recorded, tracked and applied in performance appraisals. Other sites had less formal, more traditional forms of contact between line management and shop floor personnel.

\section{- PPE Compliance}

Participating sites deployed PPE compliance surveys on a sporadic basis. Those with formal audit programs usually had an element dealing with the extent of use of PPE, along with selection, storage and maintenance aspects. This is an area of opportunity for most sites.

\section{- Promotional Efforts for OHS}

Most sites were able to cite examples of promotional efforts that had been carried out in the past. Some sites had a program of activities for the year, with a different theme every month. Others had more intermittent efforts. 
Some sites used a "top down" approach, while others used more participative strategies to promote and pursue their safety objectives.

\subsubsection{Scoring and relationships between variables}

Earlier, reference was made to the high level of diversity that exists within the study group. This applies in relation to remoteness from major population centres, labour turnover, size and complexity of the operation, number of chemicals on site, access to professional resources, sophistication of OHS programs, management style, culture, and similar.

For the present purpose the variables of interest are the number of potential benchmarking opportunities and the related "innovation score" for each facility, the Lost Time Injury Frequency Rate, and the management systems audit score as developed by the Department of Industry and Resources of Western Australia.

As mentioned earlier, the sequence and style of questions presented in the study booklets were designed to flush out potential areas of innovation. In the previous chapter, a "facility profile" was given for each participating site. This represented the elements of compliance, organisation of work, the workplace environment and people initiatives, and was derived from information collected with the aid of Booklets 3-6 and subsequent verification activities. When this information is subject to simple (unweighted) aggregation, as shown in Figure 36 , site strengths become more apparent. It can be seen that compliance scores fall in the range $73 \%-95 \%$ (mean $90 \%$ ). Organisation of work scores are in the range $28 \%$ - $91 \%$ (mean $69 \%$ ), while workplace environment scores are in the range $33 \%-100 \%$ (mean $66 \%$ ) and people initiatives are in the range $0 \%-62 \%$ (mean $31 \%$ ). The results are consistent with the observation of Wiarda and Luria (1997, p. 7) that a search for an "all-round best practice" partner is unlikely to bear fruit. No single facility is good at everything.

When compliance is put to one side, the other three elements may be used to develop an "innovation" score for each facility. This was established from the number of "Good" or "Excelient" ratings offered by 
the site representative in response to questions in Booklets 4,5 and 6. It would be reasonable to expect that, if the right questions are being asked and reliable responses are being received, then sites with many "Good" or "Excellent" ratings would have more robust and innovative programs than sites that volunteer fewer of these ratings.

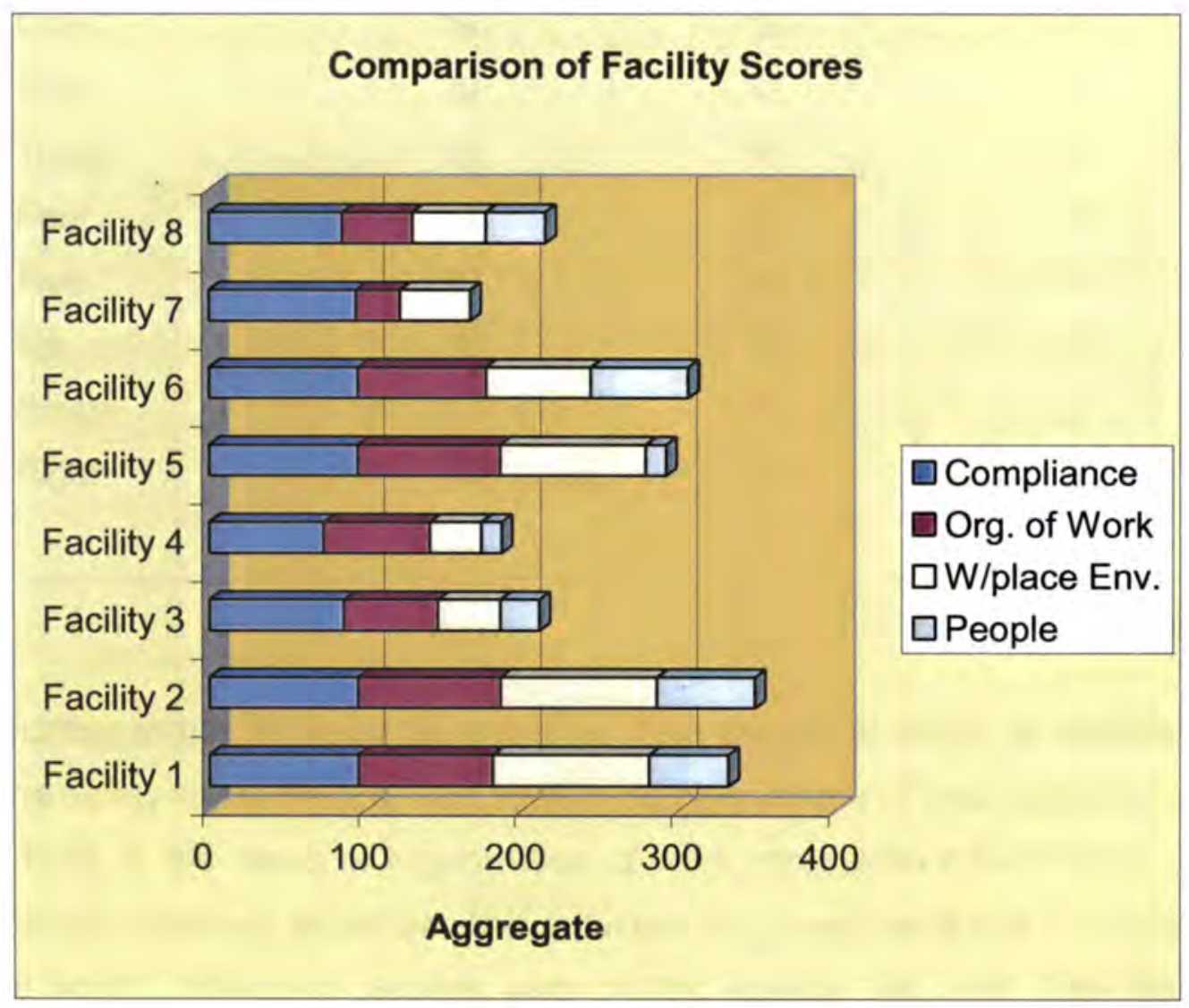

Figure 36: Make-up of Facility Scores

Table 46 presents the number of potential benchmarking opportunities for each location, as derived from the responses of interviewees to the questions raised in Booklets 4-6. Facility Two was the most prolific in this respect, with 40 nominated items out of a possible 45 .

When the scores are summed and then expressed as a percentage of the maximum possible score (45), a set of innovation scores may be developed. These are expressed in bar chart form in Figure 37. Scores ranged from $29 \%$ for Facility Seven to $89 \%$ for Facility Two. The mean score for the eight facilities was $61 \%$. 
Table 46

Number of Identified Benchmarking Opportunities

\begin{tabular}{|l|c|c|c|}
\hline \multicolumn{1}{|c|}{ Facility } & $\begin{array}{c}\text { Organisation of } \\
\text { work }\end{array}$ & $\begin{array}{c}\text { The workplace } \\
\text { environment }\end{array}$ & $\begin{array}{c}\text { People } \\
\text { initiatives }\end{array}$ \\
\hline One & 19 & 15 & 4 \\
Two & 20 & 15 & 5 \\
Three & 13 & 6 & 2 \\
Four & 15 & 5 & 1 \\
Five & 20 & 14 & 1 \\
Six & 18 & 10 & 5 \\
Seven & 6 & 7 & 0 \\
Eight & 10 & 7 & 3 \\
& & & \\
\hline
\end{tabular}

Further to this, it would be expected from the broad thrust of material in the safety literature that sites scoring highly in terms of their creativity and efforts in the areas of organisation of work, workplace environment and people initiatives would see this reflected in a lower incidence of mishaps at work. Innovation scores were plotted against the Lost Time Injury Frequency Rate (LTIFR) experienced by participating sites in 2002 to test this expectation. Figure 38 shows an inverse relationship between the two variables.

The results are suggestive of a trend (that higher levels of innovation are associated with a lower incidence of injury). However, it is difficult to draw conclusions from such a limited data set. Any relationships that may exist between the two variables would have been better explored if there had been a few poorly performing facilities within the study group. The Coal Sector of the Mining Industry of Western Australia experienced an LTIFR of 17.4 during 2001/2 (Department of Industry and Resources, 2003, p. 14 ) and may offer some opportunities in this regard. 


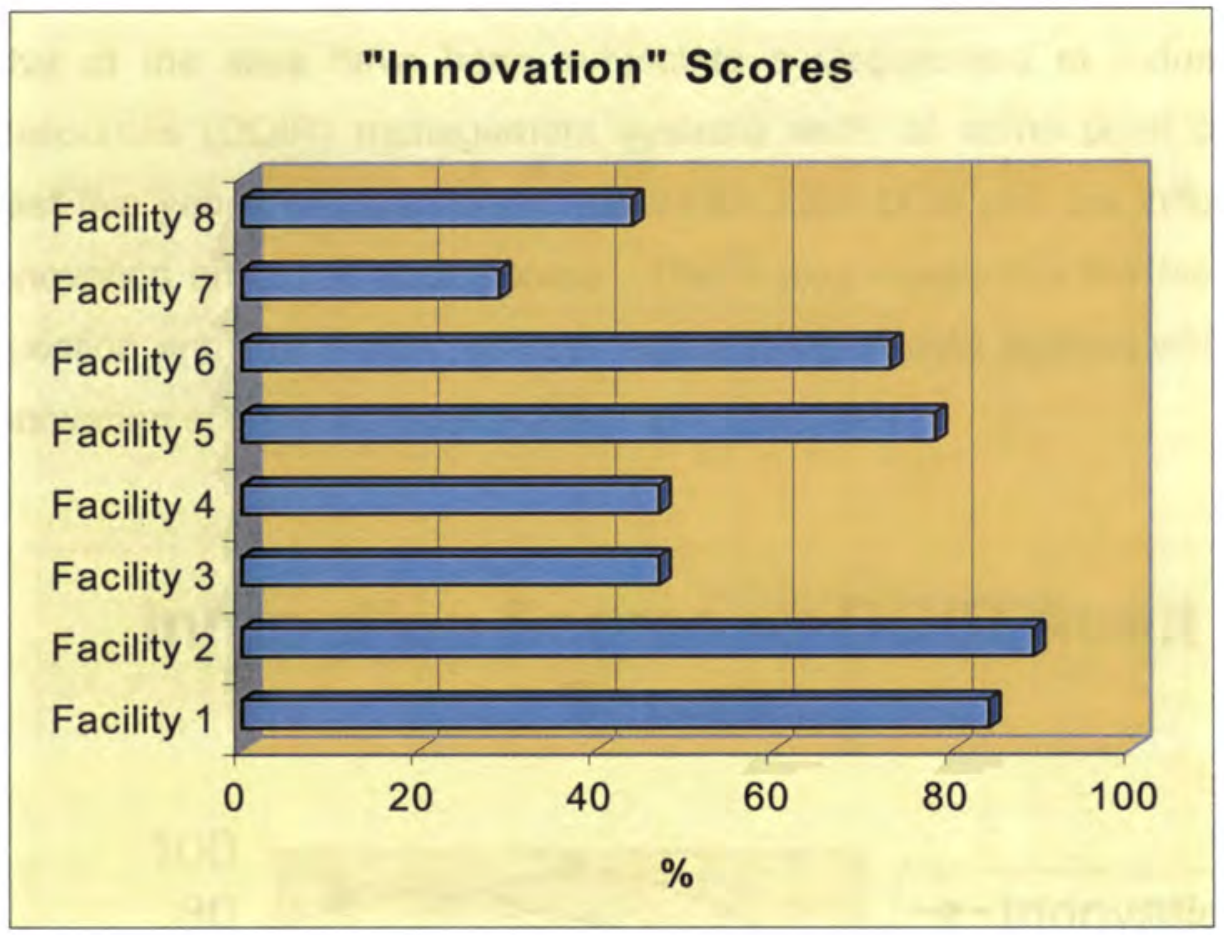

Figure 37: Innovation scores

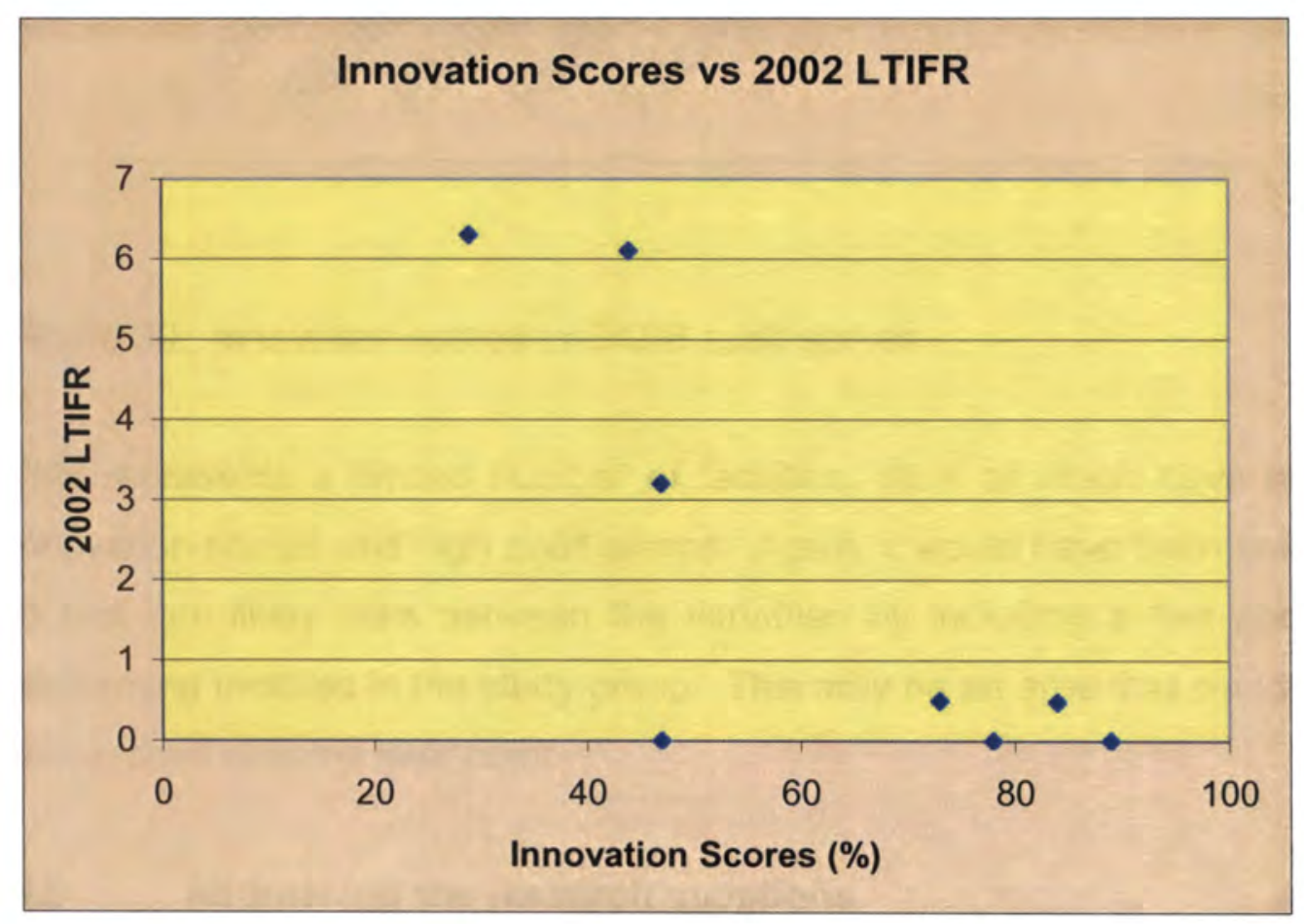

Figure 38: Innovation scores vs 2002 Lost Time Injury Frequency Rates 
Five of the sites have been subject to a Department of Industry and Resources (DOIR) management systems audit at some point over the past five years. Figure 39 represents an attempt to plot the influence of innovation on DOIR audit scores. The $X$ axis represents the facilities in question and the $Y$ axis provides a percentage scale against which both innovation scores and audit scores can be applied.

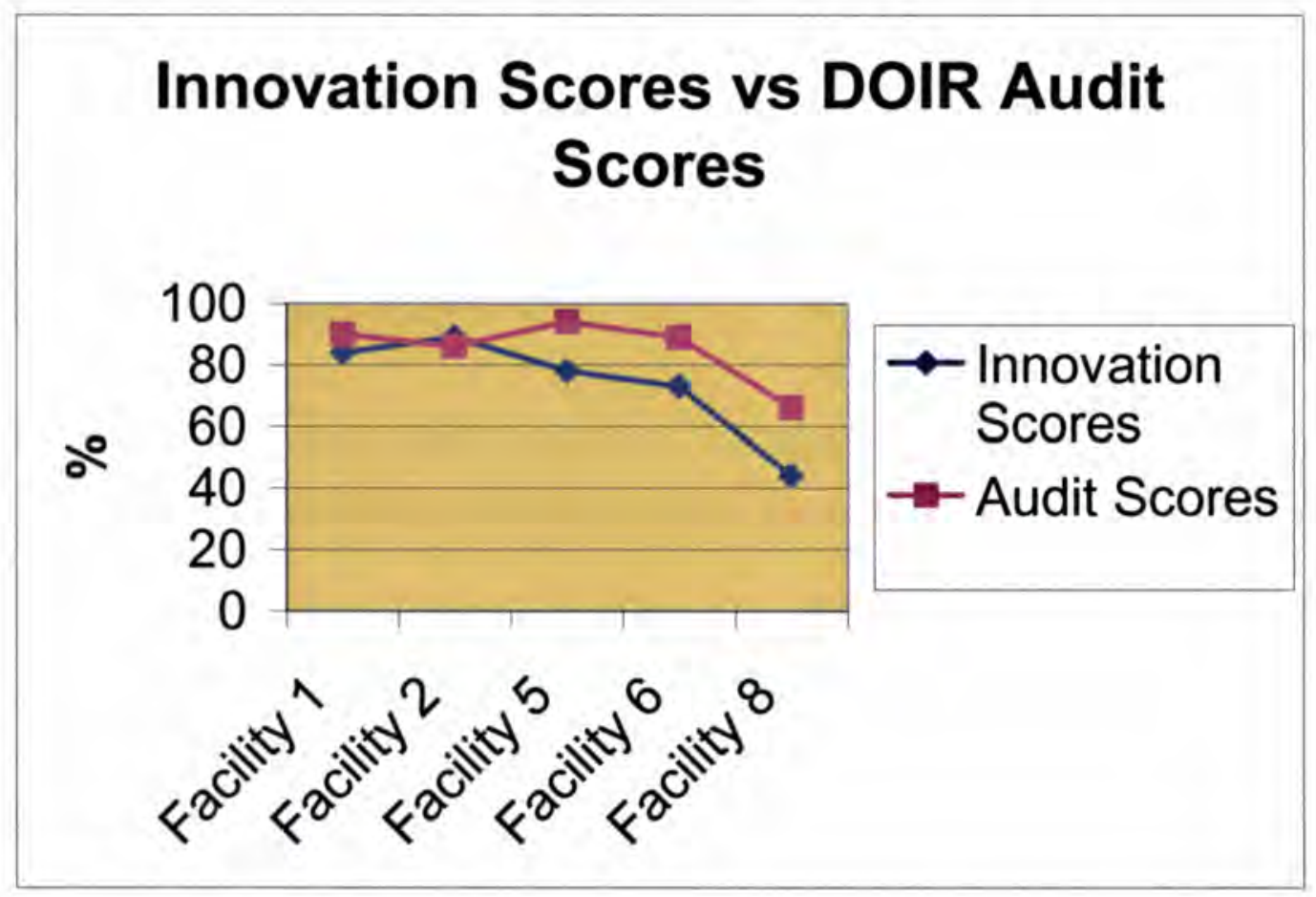

Figure 39: Innovation scores vs DOIR audit scores

This represents a limited number of facilities, most of which have high innovation scores and high audit scores. Again, it would have been useful to test any likely links between the variables by including a few poorly performing facilities in the study group. This may be an area that could be researched at some later point.

\subsection{Addressing the research questions}

Five research questions are central to this study. The questions and some comments and answers relating to each of them are provided as follows: 
- Is there a suite of benchmarking tools and meth ndologies capable of accounting for the organisation of work factor, the workplace environment factor and the people factor - as they relate to the management of hazardous materials?

The conceptual framework for this study was based on these .ee elements. There is a substantial body of literature dealing with "'? influence of each on mishaps and other OHS outcomes, and on theis $: e$ in the deployment of a strong OHS program. The booklets used to gather data at each facility dealt with each element in turn. Furthermore, the study processes were built around efforts to deploy the booklets. Thus, if the tools and processes have been effective then the research question can be answered as "yes".

- Is there a suite of benchmarking tools and methodologies capable of application to both medium and lange organisations?

It was indicated earlier that, for the purposes of this study, a "mediumsized" facility would be defined as one having 50-200 full-time employees, while a "large" facility would have in excess of 200 employees. Using these criteria, the study group consisted of four medium-sized operations (Facilities Three, Four, Five and Seven) and four large operations (Facilities One, Two, Six and Eight), thereby offering a reasonable opportunity tos tert the second research question. Evidence emerged during the study that the benchmarking tools and methodologies could be applied equally well in both medium and large facilities.

- Is there a suite of benchmarking tools and methodologies capable of application to other elements under the OHS umbrella, that is, to areas other than chemical safety?

The third research question is concemed with the transferability of the tools and processes to other elements under the OHS umbrella. This was tested in the engonomics area. The 15-page booklet, "Ergonomics - In Pursuit of Best Practice", provided in Appendix 9, proved to be relatively 
simple to produce and apply. This was a likely outcome, given that its content was modelled on the format and style of questioning that had worked well when applied with the main group of booklets. The focus group of four physiotherapists/ergonomists and an occupational physician provided further endorsement of the attributes and efficacy of this type of approach. Their response is summarised in Table 45. All four criteria were rated "Good" or "Excellent" by the practitioner panel.

- Is there a suite of benchmarking tools and methodologies capable of identifying pockets of excellence?

The fourth research question lies at the heart of the project. The principal benchmarking tools were the booklets that were used to gather preliminary information on OHS at the site (Booklets $1 \& 2$ ) and that used to work systematically through regulatory matters (Booklet 3 ), together with "beyond compliance" efforts relating to organisation of work, the workplace environment and people factors (Booklets 4-6). As highlighted earlier, these were effective in identifying "pockets of excellence".

The methodologies or processes used in this research included self-study steps and initial approaches to prospective benchmarking partners. These preliminaries were followed by a field component based broadly on the steps used in modem auditing, and described earlier. Thus, siructured interviews were used, with both formal and informai questioning being applied. Subsequently, physical examination of the workplace took place, together with document review and other types of verification. Finally, steps were taken to assess the information, to develop an accurate picture of performance at the facility and to provide feedback on strengths and opportunities for improvement.

- Is there a suite of benchmarking tools and methodologies capable of facilitating the transfer of best practice in the management of hazardous materials?

The fifth research question is different from the fourth question in that it deals with the next step in the sequence after potential benchmarks are 
identified, namely the ease with which best practice ideas or approaches may be transferred to another facility. It was possible to test only the first part of this element, by getting the subjective views of experienced OHS practitioners on the efficacy of knowledge transfer in areas of demonstrated innovation. It will be some time before the tools and processes are fully deployed, exchanges have occurred and success can be evaluated in a more comprehensive way.

\section{$5.6 \quad$ Summary}

This study has sought to develop and test new tools and processes to make benchmarking activity and the transfer of technology, ideas and approaches more efficient and meaningful. It has drawn heavily from state-of-the-art management theory and has sought to establish the linkage between the organisation of work factor (management enablers), the workplace environment factor (technical initiatives) and the people factor, as they contribute to workplace health and safety performance. It has used qualitative inquiry methodologies and an approach based on personal contact and insight, as expressed by Patton (1990, p. 46), to generate data. Information was generated by in-depth, open-ended interviews with key personnel at the facility, direct observation and review of written documents.

Some broad trends emerged from the study sites and for reasons expressed earlier are probably representative of the situation in the wider Mining Industry of Western Australia. These were:

- Most locations had good management systems and structures, including procedures and standards, although deployment was sometimes an issue.

- Most were much stronger and innovative in planning, organisation, commitment and in the other management enablers than they were in pursuing certain people-related initiatives (such as behavioural safety, management safety contacts, attitude surveys and similar) that are strongly advocated in the safety literature. 
- Most made good use of lagging indicators of performance, but were weaker in the development and application of leading indicators.

- Common opportunities for improvement were in regard to secondary labelling of containers, documentation of risk assessments, evaluation of the effectiveness of training and employee participation and ownership of the safely effort.

- Finally, while each site responded readily to the invitation to nominate critical success factors for chemical safety there were few objective measures being applied to check performance in these key areas.

The scores that were derived from the responses from the site representative and the verification activities that went with this represent an indirect indicator of innovation, activity and commitment at the site and should carry some predictive potential in terms of OHS outcomes. All of the facilities that participated in the study were reasonably strong performers in OHS, as assessed by study tools and by other independent measures, such as Government audit outcomes and widely-used lagging indicators of safety performance. All demonstrated that they had something to offer in terms of potentiai benchmarking opportunities. The five research questions raised at the beginining of this study are responded to in the affirmative. 


\section{CHAPTER 6 CONCLUSIONS AND RECOMMENDATIONS}

\subsection{Conclusions}

\subsubsection{Study objectives and outcomes}

The study set out to develop effective tools and processes for the benchmarking of hazardous materials management practices at medium and large enterprises. While there exist many sources of guidance and direction in terms of how OHS should be managed at a facility, there are clear shortfalls in terms of the tools and processes needed to identify areas of opportunity and to overcome barriers to the efficient transfer of ideas and techniques. This holds in relation to knowledge transfer between cooperating organisations, and even between locations or departments within the same organisation.

The principal findings from the study were as follows:

- The products developed for this work were tested at a selection of mining, mineral processing and related-industry sites in Western Australia. They were found to be robust and to offer considerable promise as agents of change and as support for attempts by the enterprise to achieve excellence in the management of chemical safety.

- Subject matter fell readily under the three headings represented in the conceptual framework for the study (Figure 1). All relevant material may be assigned to organisation of work, the workplace environment or people initiatives.

- The study generated evidence that the tools and processes are transferable to other critical elements of OHS management, and probably beyond that. The approach taken with the format and delivery of the booklet series seems suited to any business process that operates to standards, guidelines, codes of practice or regulatory controls - these being the principal source of the sub-elements and questions that populate the booklet for the subject being explored. 
- There appears to be a relationship between "innovation scores", as developed by the study and Lost Time injury Frequency Rate (LTIFR), the lagging indicator of OHS performance that is widely used across Australian industry.

- There were marked differences from site-to-site in terms of management style, employee ownership of OHS, program sophistication, access to professional resources, level of use of chemicals and similar factors. Nevertheless, all sites had something to offer in terms of innovations. These took many forms - variously as a product, service, tool, resource, system, concept or an approach.

- None of the sites involved in this study had participated previously in a formal, structured exchange on chemical safety (or $\mathrm{OHS}$ ) - although all sites had engaged in some level of informal exchange, usually via professional networks, meetings, conferences, and similar.

\subsubsection{Novel aspects of the study}

There is little evidence of formalised OHS benchmarking activity in Australia and so it is difficult to draw comparisons with other work. The National Occupational Health and Safety Commission (1996) benchmarking kit provides a few examples of rganisations that have set up teams and have attempted to benchmark subjects that fall under the OHS umbrella, but these appear to be scant in number. It is more likely that there is a degree of less formal contact being made between clusters of organisations from time-to-time and on quite specific items of interest. However, rarely do the outcomes of such contact find expression in the published literature.

This study is novel in that it is the first formalised, OHS-related, multi-site benchmarking effort in Western Australia. It is unique in that it addresses compliance issues, together with "beyond compliance" efforts that fall under the headings of organisation of work, the workplace environment and people factors.

It taps into current thinking and practice with respect to quality management and continuous improvement, organisational effectiveness, 
change management, behavioural safety, risk management, performance measurement, reporting and review, management systems, compliance, standards, auditing, benchmarking and technology transfer. It has sought to fashion a coherent set of tools and processes from these sources.

The study used a set of questionnaire booklets to provide for a structured coverage of the elements that fall under the OHS (general) and chemical safety headings.

Notice was taken of Webster's view, alluded to earlier, that the appearance of the questionnaire will impact greatly on the level of response to the material (Webster, 2000, p. 5). Accordingly, the questionnaires were designed and formatted to meet the criteria she calied for - namely, to demonstrate professionalism, quality and attractiveness. The booklets in Appendix 7 can be readily adapted to other elements that fall under the OHS umbrella, and so represent a resource for those who might choose to follow down this path.

Earlier, there was reference to the view by Walleck, O'Halloran and Leader (1991) that the use of benchmarking as a business improvement tool has been impeded by three obstacles, namely, the supposed superiority of invention over copying, the "we are unique" syndrome, and moral and legal sanctions against "industrial espionage". Successful benchmarking experiences of the kind described in this study should help to overcome these prejudices and encourage a lot more activity within industrial groups of like mind.

\subsubsection{Usefulness of this information}

While some benchmarking opportunities may become readily apparent in "statements of pride" in Company reports, presentations and the like, as asserted tyy Tucker, Zivan and Camp (1987, p. 9), others may only come to light as "pockets of excellence" are identified during more formal proceedings of the kind described in this work.

The information in this study and the tools and processes that have emanated from it should be of interest to: 
- Private organisations that wish to better understand their internal processes and to fast track their improvement efforts in OHS.

- Regulatory agencies that wish to promote the pursuit of best practice in OHS and are seeking some evidence of a commitment to that objective by operations that are within their jurisdiction.

- Employees and employee representatives seeking reassurance that employers and employer organisations are vigorously seeking to improve the workplace environment and reduce the incidence of injury and disease.

Materials and approaches described in this study should encourage organisations to seek out benchmarking partners and to extract the benefits that go with understanding internal business processes, sharing of ideas and fast-tracking of improvements. As OHS benchmarking in Western Australia becomes a more entrenched and recognised business practice the very processes and tools themselves will evolve to a new level of effectiveness. As Watson (1992, p. 119) puts it: "Partnering with other firms on benchmarking studies can help you expand your capability to benchmark by negotiating benchmark protocols and by observing how other companies conduct their benchmarking process.

\subsection{Recommendations}

\subsubsection{New frontiers and prospects for further work}

This study has focused on creating a means to better identify benchmarking opportunities in OHS (general) and in chemical safety. There appears to be considerable scope to further develop the tools and processes that have been described - with a view to application in other areas of OHS, such as electrical safety, machinery safety, confined space work, mobile equipment operation, work at heights and similar.

Some of the papers consulted during the literature review phase refer to issues and developments worthy of follow-up. The researcher was keenly interested in the bar code fechnology being applied at the Jet Propulsion Laboratories in Pasaderia, California and described by Houseman, Behar 
and Le Blanc (2001). This has the potential to greatly enhance the way chemical inventories are managed on site and to reduce the likelihood of chemical-related injury or disease outcomes.

In recent years, hand-held Personal Digital Assistants (PDAs) have proved to be versatile and powerful business aids. Increasingly, they are being used in field situations as tools for applying audit or inspection checklists, for verification activities, for recording of observations and for other forms of data gathering. Data collected in this way may then be readily uploaded to a laptop computer for further analysis and for preparation of reports (C. Cumming, personal communication, February 8,2003 ). There appears to be potential for the PDA technology to be applied to some of the benchmarking elements described in this study - so this represents a potential area for future research and development.

Along similar lines, the forms that have been used in this study would appear to lend themselves to computer-based analysis. This could be developed such that the user could answer the questions on-line, with the program collecting, recording and processing the data instantly ( $G$. Robinson, personal communication, February 21, 2003).

\subsubsection{Next steps}

Given the outcomes that have been described above, it will be important that others are able to access the details and deploy similar approaches, where appropriate, to their business improvement strategies.

For the researcher, the immediate task is to share information with the Mining Industry in Western Australia. This may be accomplished quite readily by making contact with key elements of the industry, namely the OHS Committee of the Chamber of Minerals and Energy, the State Mining Engineer and staff at the Department of Industry and Resources, and the tripartite structures that handle OHS issues for the Industry, such as the Mines Occupational Safety and Health Advisory Board.

Strong expressions of interest in the tools and techniques of this study have been received from member companies of the Kwinana Industries 
Council. The facilities that operate in the Kwinana industrial area have a record of using cooperative and supportive approaches to EHS matters. a willingness to look at new ideas and have the advantages of geographical proximity.

There is a further responsibility to reach the wider OHS community in Australia and elsewhere. This may be accomplished by publishing key findings and outcomes in widely read publications such as the World Safety Journal or the Journal of Occupational Health and Safety - Australia and New Zealand.

There has been some early interest in the tools and processes from contacts outside Australia. For example, study materials are currently being evaluated at a closures plant in Tianjin, China. If trials are successful, the materials will be translated into the Mandarin language and used more widely in that country.

Benchmarking techniques and processes are powerful aids in the pursuit of best practice and in the continuous improvement efforts being applied by many businesses around the world. Hopefully, the research work described in this document will stimulate greater and more meaningful application of benchmarking to health and safety in the workplace.

"Fear not to touch the best"

(Sir Walter Rajeigh, c. 1552 - 1618) 


\section{REFERENCES}

Adams, E.E. (1991, August). The quality revolution - a challenge to safety professionals. Professional Safety, 22-28.

Adams, B., \& Adams, C. (1995). Corporate DNA: The genetic code. Executive Excellence, 12(10), 8-9.

Alcoa. (1990). Excellence through quality - benchmarking. Pittsburgh, Pennsylvania: Author.

Alcoa. (1995). Health and safety management system guide: Training tools. Pittsburgh, Pennsylvania: Author.

Alcoa. (1997). Alcoa's environment, health and safety management system: February 1997 Draft. Pittsburgh, Pennsylvania: Author.

Alcoa. (1999a). 1999 annual review: Environment, health and safety update. Perth, Western Australia: Author.

Alcoa. (1999b). Alcoa audit: Future direction. Pittsburgh, Pennsylvania: Author.

Alcoa. (1999c). Special auditor training. Perth, Western Australia: Author.

Alcoa. (2000). Self assessment news. Pittsburgh, Pennsylvania: Author.

Alcoa. (2001a). Alcoa ergonomics standard. Pittsburgh, Pennsylvania: Author.

Alcoa. (2001b), Alcoa in Australia: 2000 annual review. Perth, Western Australia: Author.

Alcoa. (2002a). Environment, health and safety risk management, draft internal mandated standard, December 2002, Pittsburgh, Pennsyivania: Author. 
Alcoa. (2002b). Balanced scorecard: OHS, December 2002, Powerpoint presentation. Perth, Western Australia: Author.

Allaire, P.A. (1991, March). Quality: where it has been and where it is going. Journal for Quality and Participation, 64-66.

Alpander, G.G., \& Lee, C.R. (1995). Culture, strategy and teamwork. Journal of Management Development, 14(10), 4-18.

American Board of Industrial Hygiene. (1984). Bulletin of the American Board of Industrial Hygiene. Akron, Ohio: Author.

American Psychological Association. (2001). Publication manual of the American Psychological Association (5 $5^{\text {th }}$ ed.). Washington, DC: Author.

Amis, R.H., \& Booth, R.T. (1992, February). Monitoring health and safety management. The Safety \& Health Practitioner, 43-46.

Anderson, J., \& Poole,M. (1998). Assignment and thesis writing. Brisbane, Queensland: John Wiley \& Son.

Anon. (2003). Toxic Substances Control Act (USA) [on-line]. Retrieved January 7, 2003 from http://www.ohs.netnam.vn/html

ANZMEC. (1999). Realising a safe and healthy mining industry Developing a strategic framework for Government's contribution (in draft). Australian and New Zealand Minerals and Energy Ministerial Council Conference, 27-29 October, 1999. Adelaide, South Australia: Author.

Arnold, R.M. (1992, April). Measuring the health of your safety audit system. Professional Safety, 46-49.

Aune, A. (1991). A recipe for success. Total Quality Management, 3(1), 33-37.

Australian Manufacturing Council. (1994). Leading the way - A study of best manufacturing practices in Australia and New Zealand. $2^{\text {nd }}$ 
Edition. Melbourne: Author.

Bailey, C.W., \& Petersen, D. (1989, February). Using perception surveys to assess safety system effectiveness. Professional Safety, 22-26.

Baldridgeplus.com (1999). Balanced scorecards - exhibit. Retrieved June 6, 2000, from http:/www.Baldridgeplus.com/Exhibits.pdf

Bea, R. (1999, November). The next steps: advancing the causes of quality, reliability and safety. Proceedings of the Workshop on Behavioural Change and Safefy. 24 November, 1999. Perth, Western Australia: Woodside Offshore Petroleum Pty. Ltd.

Blake Dawson Waldron. (2001, December). A review of Longford - the OHS prosecution. Safety Matters.

Blewett, V. (1994). Beyond lost time indicators: Positive performance indicators for OHS - summary paper. In Positive performance Indicators: Beyond lost Time Injuries, Part 1 - Issues, Sydney, NSW: Worksafe Australia.

Blewett,V., \& Shaw, A. (1995a). Integrating OHS through self-managed work teams. J. Occup. Health and Safety-Aust. NZ, 11(1), 15-19.

Blewett, V., \& Shaw, A. (1995b). Benchmarking OHS: a tool for best practice. J. Occup. Health and Safety - Aust. NZ, 11(3), 237-242.

Blewett, V., \& Shaw, A. (1996a). The OHS professional: manager of change or changing manager? J. Occup. Health and Safety - Aust. NZ, $12(1), 49-54$.

Blewett, V., \& Shaw, A. (1996b). Telling tales: OHS and organisational culture. J. Occup. Health and Safety - Aust. NZ, 12(2), 185-19\%.

Blewett, V., \& Shaw, A. (1996c). Quality occupational health and safety? J. Occup. Health and Safety - Aust. NZ, 12(4), 481-487.

Blewett, V., \& Shaw, A. (1996d). Strategy for best practice in OHS. J. 
Occup. Health and Safety - Aust. NZ, 12(6), 731.737.

Bottomley, B. (1994). Means of encouraging best practice in occupational health and safety. In Positive Performance Indicators Beyond Lost Time Injuries, Part 2-Practical Approachos, Sydney, NSW: Worksafe Australia.

Brandt, M.T. (1997, March). Managing change in health and safety departments. The Synergist, 25-28.

British Standards Institution. (1996). BS 8800: Guide to Occupational Health and Safety Management Systems. London, UK: Author.

Business Excellence Australia. (2003). Australian business excellence awards. Retrieved January 2, 2003 from http://www.businessexcellenceaustralia.com.au/GROUPS/AWARDS/

Butcher, C., \& Pennicuik, S. (1999). The 1995 ACTU national hazardous chemicals campaign. J. Occup. Health Safety - Aust. NZ, 15(2), 131136.

Calder, A. (1996). Practical application of auditing to enhance health and safety performance. In Papers presented at Minesafe Intemational 1996, Perth, Western Australia: Chamber of Minerals and Energy, 369-378.

Calder, A., \& Davies, M. (1996). What do dashboarơs have to do with measuring safety? In Papers presented at Kinesafe Intemational 1996, Perth, Western Australia: Chamber of Minerals and Energy of WA, 325-331.

Cane, A., (1995, January 20). Getting the best out of people is the buzz, empowerment is the word. The Financial Review, p. 20.

Chamber of Commerce and Industry of WA. (1999, August 31). World congress on occupational safety and health, Bulletin - HR Link. 
Chamber of Minerals and Energy of WA. (1993). Health, Safety and Environment - Self Audit System, Perth, Western Australia: Author.

Chesson, B.J. (1990). Materials information: meeting the needs of the workplace. J. Occup. Heaith and Safety - Aust. NZ, 6(5), 405-411.

Chesson, B.J. (1995). The hazardous substances package - an industry viewpoint. Safety Institute Joumal, 2(4), 18-20.

Chesson, B.J. (1999). The Esso Longford gas plant accident - an opportunity to learn. In Papers presented at the Corporate Risk Management Conference, 20 October, 1999. Perth, Western Australia.

Claridge, G. (1999). Managing for outcomes - impacts and intervention logic. Workshops for public service departments, 22/23 October, 1999. Retrieved January 13, 2003 from http://io.ssc.govt.nz/pathfinder/

Cook, D., \& Blaxter, T. (1991, June). Maintaining momentum. Total Quality Management, 149-151.

Davison, L. (2000, April). Searching for information on the Internet. The Synergist, 15-17.

Dare, P. (1995, April). Auditing for COSHH. Croner Hazardous Substances Briefing, Issue No. 38, Croner Publications, 3-4.

Deacon, A., \& Pearce, J. (1992, July). Managing safety: introducing QSA. Occupational Safety \& Health, 14-15.

Deacon, A. (1994, January). The role of safety in total quality management. The Safety and Health Practitioner, 18-21.

Deming, W.E. (1986). Out of the crisis. Cambridge, Mass: Institute of Technology, Centre for Advanced Engineering.

Department of Consumer and Employment Protection. (2003). State of the work environment statistics 2002. Retrieved February 13, 2003 from http://umw.safetyline.wa.gov.au/pagebin/injrstat0309.pdf 
Department of Industry and Resources. (2003). Safety performance in the Western Australian mineral industry 2001/2002 - Accident and Injury statistics. Perth, Western Australia: Author.

Dial, C.M. (1992, April). Incident-focused managers. Professional Safety, $37-43$.

Department of Minerals and Energy. (1994). Mines Safety and Inspection Act 1994. Perth, Westem Australia: Author.

Department of Minerals and Energy. (1995a, December 8). Mines Safety and Inspection Regulations 1995, Extract from Government Gazette (No. 169). Perth, Western Australia: Author.

Department of Minerals and Energy. (1995b, September). "If you always do what you always did, you'll always get what you always got". Minesafe, 6(3), Perth, Western Australia: Author.

Department of Minerals and Energy. (1996, September). Safety culture: part two. Minesafe 7(3). Perth, Westem Australia: Author.

Department of Minerals and Eneryy. (1997a). Guideline - Management of Hazardous Substances on Minesites. Perth, Western Australia: Author.

Department of Minerals and Energy. (1997b). Management Systems Audit Guideline Document. Perth, Western Australia: Author.

Department of Minerals and Energy. (1998, September). Safety culture (as reflected by behaviour). Minesafe, $9(1)$. Perth, Western Australia: Author.

Department of Minerals and Petroleum Resources. (2002). Safety performance in the Westem Australian Mineral Industry 2000/2001 Accident and Injury Statistics. Perth, Western Australia: Author.

Department of Occupational Health, Safety and Welfare of WA. (1994a). Jobsafe Plan: WA's Assessment of Occupational Health and Safety Management Systems - Proliminary Self Assessment. Perth, Western 
Australia: Author.

Department of Occupational Health and Safety of WA. (1994b). Making the Workplace Safe - Occupational Health and Safety Laws. Perth, Western Australia: Author.

Dyjack, D.T., \& Levine, S.P. (1995). Development of an ISO 9000 compatible occupational health standard: defining the issues. Am. Ind. Hyg. Assoc. J., 56, 599-609.

Eastman Kodak. (1994). Safety performance indexing: Metrics for safety performance improvement projects. Corporate Safety. Rochester: Autior.

Edith Cowan University. (2003). Doctoral and masters by research handbook: Information for candidates and supervisors. The Graduate School. Perth: Author.

Environmental Manager. (1992, August). Surveys can assess safety program effectiveness. Environmental Manager, 9-14.

Erivironmental Manager. (1995, January). A new system for human error management. Environmental Manager, 11-13.

Farnell, G. (1991, March). A behavioural approach to safety management. Journal of Health and Safety, (6), 39-44.

Feigenbaum, A.V. (1991, March). Quality: an international imperative. Journal for Quality and Participation, 16-18.

Fisher, T. (1991). A. "quality" approach to occupational health, safety and rehabilitation. J. Occup. Health and Safety - Aust. NZ, 7(1), 23-28.

Fulwiler, R.D. (1998, January). The application of be raviour-based management systems to industrial hygiene. The Synergist, 26-27.

Gallacher, H. (1991, June). Avoiding the piffalls: Total quality management, 157-160. 
Geller, E.S. (1997a, May). Seven principles of systems thinking. Industrial Safety and Hygiene News Magazine, 1-4.

Geller, E.S. (1997b, October). Key processes for continuous safety improvement. Professional Safety, 40-44.

Geller, E.S. (1998). Working safe - how to help people actively care for health and safety. Florida: CRC Press.

Geller, E.S. (1999a). Three keys to safety success: No. 1 in a series. Safety WA. Perth, Western Australia: Industrial Foundation for Accident Prevention.

Geller, E.S. (1999b). Quality recognition for safety improvement: No. 2 in a series. Safety WA. Perth, Western Australia: Industrial Foundation for Accident Prevention.

Geller, E.S. (1999c). Do you invest in property damage?: No. 3 in a series. Safety WA. Perth, Western Australia: Industrial Foundation for Accident Prevention.

Geller, E.S. (1999d). Which should be changed first - attitude or behaviour?: No. 4 in a series. Safety WA. Perth, Western Australia: Industrial Foundation for Accident Prevention.

Geller, E.S. (1999e). Increasing the impact of signs and slogans: No. 6 in a series. Safety WA. Perth, Western Australia: Industrial Foundation for Accident Prevention.

Geller, E.S. (1999f). Avoid the seven common pitfalls: No. 7 in a series. Safety WA. Perth, Western Australia: Industrial Foundation for Accident Prevention.

Geller, E.S. (1999g). Fuelling the participation factor: No. 8 in a series. Safety WA. Perth, Western Australia: Industrial Foundation for Accident Prevention.

Gerhardsson, G. (1998, January). How to boost occupational health and 
safety's visibility worldwide. AlHA Journal, 59, 6-7.

Glendon, I., \& Booth, R. (1995). Risk management for the 1990s:

measuring management performance in occupational safety and health.

J. Occup. Health and Safety - Aust. NZ, 11(6), 559-565.

Glendon, I. (1995). Risk management for the 1990s: safety auditing. $J$. Occup. Health and Safety - Aust. NZ, 11(6), 569-575.

Gray-Spence, E. (1994). Positive OHS performance indicators. In Papers from Worksafe Workshop on Positive Performance Indicators, 19 May 1994, Sydney, NSW.

Griffiths, D.J. (2000). The promotion of PPls as a means of accelerating the rate of $\mathrm{OH} \& S$ performance improvements. In Papers from the Positive Performance Measures for Workplace OH\&S Conference, 8-9 November 2000, Sydney, NSW.

Hartley,M. (2000, June). Managing chemicals better for occupational health. Chemistry in Australia, 8-10.

Harvey, B. (Ed.) (1980). Handbook of occupational hygiene, Vol. 1, 2 and 3. Kingston-upon-Thames, UK: Kluwer/Croner,

Health and Safety Executive (1992). Successful health \& safety management. London, UK: HMSO.

Health and Safety Executive. (1999). Health and safety benchmarking: improving together. (INDG301 9/99 C1000). Sunbury, UK: HSE Books.

Herbert, D.A. (1994). Measuring safety and identifying trends in safety performance. In Papers presented at the OSH '94 Conference and Trade Show. Toronto, Canada.

Holland, S., \& McEwan, M. (1999). The national hazardous substances regulatory framework: first revisions now available. J. Occup. Health \& Safety - Aust. NZ, 15(2), 127-130. 
Houseman, J., Behar, J. L., \& LeBlanc, R. M. (2001, May). Safety controls on electronic procurement of hazardous chemicals. The Synergist, 26-28.

Howell, J., Spickett, J., \& Hudson, K. (1998). The management of hazardous substances in small business in Western Australia. $J$. Occup. Health and Safety - Aust. NZ, 14(5), 457-463.

Hurst, N.W. (1998). Risk assessment - the human dimension. Cambridge, UK: The Royal Society of Chemistry.

International Labour Office. (1990). Safety in the use of chemicals: Convention No. 170. Geneva, Switzerland: Author.

Irwin, B.M. (1994). Cultural imprints. Melbourne, Victoria: Australian Quality Council.

Isaac, S., \& Michael, W.B. (1981). Handbook in research and evaluation. $\left(2^{\text {nd }}\right.$ ed.). San Diego, USA: EdITS.

Jansz, J. \& Nedved, M. (2002). OHS 6202: Independent project study guide. Churchlands, Western Australia: Edith Cowan University.

Jay, A. (1996). The Oxford dictionany of political quotations. Oxford, UK: Oxford University Press.

Jay, C. (1994, November 2). Benchmarking identifies gaps, but doesn't eliminate them. Financial Review, p. 34.

Johnson, J. (1991, December). The culture clock. Journal for Quality and Participation, 10-13.

Johnson, S. (1988, June). Management accountability for safety performance. Professional Safety, 23-26.

Jongeling, S.B. (Ed.). (2003). Referencing guide (5th rev. ed.). Perth, Westem Australia: Edith Cowan University, Learning and Development Services. 
Juran, J. (1991, March). Made in USA - a quality resurgence. Joumal for Quality and Participation, 6-8.

Keeves, R. (1996). The search for best practice: The best practice program. Australian Federal Government. Retrieved February 12, 2000 from http://www.business.com.au/ibc/bestprac/best.htm.

King, E. (1990). Occupational hygiene aspects of biological monitoring. Ann. Occup. Hygiene, 34(3), 315-322.

Kletz, T.A. (1993). Accident data - the need for a new look at the sort of data that are collected and analysed. Safety Science, 16, 407-415.

Kotter, J.P. (1996). Leading change. Boston: Harvard Business School.

Krause, T.R., \& Hidley, J.H. (1989, October). Behaviorally-based safety management: parallels with the quality improvement process. Professional Safety, 20-25.

Krause, T.R., Hidley, J.H., \& Hodson,S.J. (1991, June). Measuring safety performance: the process approach. Occupational Hazards, 53(6), 4952.

Krause, T.R. (1997, October). Trends and developments in behaviourbased safety. Professional Safety, 20-25.

Krause, T.R. (2000). The role of behaviour-based safety in the workplace. In Papers presented at the Minesafe International 2000 Conference, Perth, September, 2000, Chamber of Minerals and Energy of Western Australia.

Laing R. (2002). Final Report: Review of the Occupational Safety and Health Act 1984. Perth, Western Australia.

Liberty Mutual Insurance Company. (2000a). Performance technology: behavioral safety progress mapping. Retrieved March 3, 2000 from http:/www.liberlymutual.com/business/safety/performance/progress _mapping.htmil 
Liberty Mutual Insurance Company. (2000b). Performance technology: corporate climate review. Retrieved March 3, 2000 from http://www.libertymutual.com/business/safety/performance/corp_climate _review.html

Liberty Mutual Insurance Company. (2000c). Performance technology: Behavioral safety readiness assessment. Retrieved March 3, 2000 from $\mathrm{http} / / \mathrm{www}$.libertymutual.com/business/safety/performance/ readiness.bbs. html

Lindsay, F.D. (1992). Successful health and safety management - the contribution of the management audit. Safety Science, 15, 387-402.

Lindsay, W.M., Manning, G.E., \& Petrick, J.A. (1991, March). Mapping work group morale. Journal for Quality and Participation, 100-107.

Lockwood, A. (1994, November). Getting the best out of benchmarking. Occupational Health Magazine, 3.

Madsen, D. (1992). Successful dissertations and theses. ( $2^{\text {nd }}$ ed.). San Francisco: Josse-Bass.

Malachowski, M.J. (1995). Health effects of toxic substances.

Rockville, Maryland: Government Institutes Inc.

Marcombe, J.T., Krause, T.R., \& Finley, R.M. (1993, April). Behaviourbased safety at Monsanto's Pensacola plant. The Chemical Engineer, $29,15-17$.

McConnell, J. (1989). The seven tools of TQC. ( $3^{\text {rd }}$ ed.). Maryborough: The Book Printer.

McGinley, M.A. (1996). ISO management models: panacea or paradigm? The Synergist, 7(9), 30-31.

Merry, M. (1998). Assessing the safety culture of an organisation. J. Safety and Reliability Society, 18(3), 14-31. 
Meyer, C. (1994, May-June). How the right measures help teams excel. Harvard Business Review, 95-103.

Minerals Council of Australia. (1997). Minex awards 1997 - Awards criteria and application guidelines. Canberra, ACT: Author.

Minerals Council of Australia. (2002). Safety and Health Performance Report: 2001-2002. Canberra, ACT: Author.

Minerva Educational lnstitute. (1991, April). Management's safety and health imperative: eight essential steps to improving the work environment. Am. Ind. Hyg. Assoc. J., 52(4), A218-221.

Mitchell, M. (1993ョ, May). If it ain't broke ... Client Newsletter. Riverstone, NSW: Mitch Mitchell and Associates.

Mitchell, M. (1993b). The TQM Safety System, Riverstone, NSW: Mitch Mitchell and Associates.

Mitchell, M. (1993c). The TQM Approach to Managing Health, Safety and the Environment. Seminar Notes. Riverstone, NSW: Mi:ch Mitchell and Associates.

Mitchell, R. (2000). Measuring OHS performance and developing performance indicators. J. Occup. Health and Safety - Aust. NZ, 16(4): 319-323.

Mines Occupational Safety and Health Advisory Board. (1999). Safety and health risk management guideline. Perth, Western Australia: Department of Minerals and Energy.

Motzko, S.M. (1989, August). Variation, system improvement, and safety management. Professional Safety, 17-20.

Mulhausen, J. R. \& Damiano, J. (1998). A strategy for assessing and managing occupational exposures ( $2^{\text {nd }} \mathrm{ed}$.). Fairfax: AlHA Press.

Munro, R.A. (1991, December). Using quality improvement to improve 
quality training. Journal for Quality and Participation, 74-76.

Mussett, J. (2000). Forward to safe business, good business. In T. Barrett \& D. Cameron (Eds.), In Safe Business, Good Business: A practical guide to occupational safety, health and insurance in Australasia (pp. i-iii). Guildford, Western Australia: Vineyard Publishing.

National Industry Extension Service. (1993). Benchmarking self help manual - Your organisation's guide to achieving best practice.

Department of Industrial Relations and the Australian Manufacturing Council. Canberra: AGPS.

National Occupational Health and Safety Commission. (1990). Storage of chemicals: Guidance note for placarding of stores and guidance note for emergency services manifests. NOHSC: 3009 and 3010 . Canberra: AGPS.

National Occupational Health and Safety Commission. (1991a). National policy statement on the commercial confidentiality of data relating to workplace subsiances. NOHSC: 6004 (1991). Canberra: AGPS.

National Occupational Health and Safety Commission. (1991b). Guidance note for completion of a material safety data sheet. NOHSC: 3001 (1991). Canberra: AGPS.

National Occupational Health and Safety Commission. (1991c). Guidance note for determining and classifying a hazardous substance. NOHSC: 3011 (1991). Canberra: AGPS.

National Occupational Health and Safety Commission. (1994a). National model regulations for the control of workplace hazardous substances. NOHSC: 1005 (1994). Canberra: AGPS.

National Occupational Health and Safety Commission. (1994b). National code of practice for the control of workplace hazardous substances. NOHSC: 2007 (1994). Canberra: AGPS. 
National Occupational Health and Safety Commission. (1994c). National code of practice for the labelling of workplace substances. NOHSC: 2012 (1994). Canberra: AGPS.

National Occupational Health and Safety Commission. (1994d). Guidance note for the assessment of health risks arising from the use of hazardous substances in the workplace. Canberra: AGPS.

National Occupational Health and Safety Commission. (1994e). National code of practice for the preparation of material safety data sheets. Canberra: AGPS.

National Occupational Health and Safety Commission. (1994f). List of designated hazardous substances. NOHSC: 10005 (1994). Canberra: AGPS.

National Occupational Health and Safety Commission. (1994g). Guidance note for the control of workplace hazardous substances in the retail sector. NOHSC: 3018 (1994). Canberra: AGPS.

National Occupational Health and Safety Commission. (1995a). Exposure standards for atmospheric contaminants in the occupational environment. 3rd Edition. Guidance Note NOHSC: 3008 (1995) and National Exposure Standards NOHSC: 1003 (1995). Canberra: AGPS.

National Occupational Health and Safety Commission. (1995b). Control of workplace hazardous substances Part 2 - scheduled carcinogenic substances. National Model Regulations NOHSC: 1011 (1995) and National Code of Practice NOHSC: 2014 (1995). Canberra: AGPS.

National Occupational Health and Safety Commission (1996a).

Benchmarking occupational health and safety. Retrieved May 15, 2000 from http: //www.worksafe.gov.au/fulltext/docs/h3/02697-01.htm

National Occupational Health and Safety Commission. (1996b). Control of major hazard facilities. National Standard NOHSC: 1014 (1996) and National Code of Practice NOHSC: 2016 (1996). Canberra: AGPS. 
National Occupational Health and Safety Commission. (1999). Approved criteria for classifying hazardous substances. Revised Edition. NOHSC: 1008 (1999). Canberra: AGPS.

National Occupational Health and Safety Commission. (2001). Occupational health and safety management systems - A review of their effectiveness in securing healthy and safe workplaces. Sydney: Author.

Nedved, M. (1999a). Safety auditing in the mining and mineral processing industries. Australian National Safety Journal, 6(4), 9-15.

Nedved, M. (1999b). Safety auditing in the mining and mineral processing industries - development of audit protocols. Australian National Safety Joumal, 7(1), 9-14.

Nedved, M. (1999c). Safety auditing in the mining and mineral processing industries - development of self-audit systems. Australian National Safety Joumal, 7(2), 9-13.

Nedved, M. (2000). Safety auditing in the mining and mineral processing industries - development of positive safety performance indicators. Australian National Safety Joumal, 7(3), 8-11.

Neufeldt, V. \& Guralnik, D.B. (Eds) (1988). Webster's New World Dictionary, Third College Edition. New York: Webster's New World.

Ojanen, K., Seppala, A., \& Aaltonen, M. (1988). Measurement methodology for the effecis of accident prevention programs. Scandinavian Joumal of Work Environment and Health, 14, 95-96.

Olishifski, J.B. \& Plog, B. A. (1988). Overview of Industrial Hygiene. In B.A. Plog (Ed.), Fundamentals of Industrial Hygiene ( $3^{\text {rd }}$ ed., pp. 3-28). National Safety Council, USA.

O'Neill, P.H. (1991, May/June). The quality struggle - from two angles. Management Strategy, 51-55. 
Oxenburgh,M. (1991). Increasing productivity and profit through occupational health and safety. Sydney: $\mathrm{CCH}$ International.

Pardy, W. (1991, May). Out with the old. Accident Prevention $n_{: 11-16 .}$

Parker, L. (Ed.) (1998, October). Hazardous chemicals treaty. Croner Hazardous Substances Briefing, Issue No. 80, 1-4.

Parker, L. (Ed.) (1999a, March). Chip 99. Croner Hazarcious Substances Briefing, Issue No. 85, 1-4.

Parker, L. (Ed.). (1999b, April). New COSHH regulations. Croner Hazardous Substances Briefing, Issue No. 86, 1-4.

Patton, M.Q. (1990). Qualitative evaluation and research Methods ( $2^{\text {nd }}$ ed.). California: Sage Publications.

Perdue, S.R. (2000, February). Observations on safety culture. Safety WA, 2-3.

Petersen, D. (1991, August). Safety's paradigm shift. Professional Safety, 47-49.

Petersen, D. (1997, October). Accountability, culture and behaviour. Professional Safety, 45.

Pidgeon, N.F. (1991). Safety culture and risk management in organisations. J. Cross-Cultural Psychology, 22(1), 129-140.

Pope, W.C. (1992). Managing for performance perfection - the changing emphasis. Weaverville: Bonnie Brae Publications.

Pryor, L.S. (1989, November/December). Benchmarking: a selfimprovement strategy. The Journal of Business Strategy, . 28-32.

Quinlan, M. (1999, May). Safety flies in the face of reward schemes. CCH's Australian OHS, 19-21. 
Ragan, P.T. (1997, October). Safety's silver bullet. Professional Safety, 26-31.

Ralph, M. (1999, June). Lessons from the E. Scott Geller seminars. Safety WA. Perth, Western Australia: Industrial Foundation for Accident Prevention.

Reason, J. (2000, March). Beyond the limitations of safety systems. Australian Journal of Mining, 41.

Redinger, C. (1997, February). BS 8800: 1996 - a British occupational health and safety management system. The Synergist, 32-33.

Redinger, C.F., \& Levine, S.P. (1998). Development and evaluation of the Michigan occupational health and safety management system assessment system assessment instrument: a universal OHSMS performance measurement tool. American Industrial Hygiene Association Journal, (59), 572-581.

Reynolds, S. (1998, July). Don't sinift blame in behavioural safety. Safety $W A, 8$.

Robens, A. (1972). Report of the committee on safety and health at work 1970-1972, London, UK: HMSO.

Roberts, P. (1996, March 28). Team playing - it's easier in theory. The Australian Financial Review, p.13.

Robson, M. (1991, March). TQM, the easy sell - the hard reality. Journal for Quality and Participation, 36-40.

Roughton, J. \& West, M. (2000, November). Safety incentives: are they worth the gamble? The Synergist, 27-30.

Sarantakos, S. (1993). Social research. South Melbourne, Victoria: MacMillan Education Australia. 
Sarazen, S.J. (1991, September). Continuous improvement and innovation. Joumal for Quality and Participation, 34-65.

Saunders, I. (1995). Managing quality and risk. J. Occup. Health and Safety - Aust. NZ, 11(6), 579-586.

Savage, E.W. (1991, September). Total quality is total change. Journal of Quality and Participation, 100-104.

Scott, I. (1999, February). DuPont shows how safety can save hurt and money, Safety WA, 8.

Shaw, A. (1994). OHS performance indicators for benchmarking - Report on the literature review conducted as stage 1 of the Worksafe Australia project to develop a benchmarking methodology for occupational health and safety. Sydney, NSW: Worksafe Australia.

Sprouster, J. (1994, November 2). New psyche puts our business in more competitive position. Financial Review, p. 30.

Standards Australia. (1997). Australian/New Zealand standard: Occupational health and safety management systems - General guidelines on principles, systems and supporting techniques. AS/NZS 4804: 1997. Homebush, NSW: Author.

Standards Australia. (1999). Australian standard: Risk management. AS/NZS 4360: 1999. Strathfield, NSW: Author.

Standards Australia. (2000). Australian standard: Occupational health and safely management systems - Specification with guidance for use. AS/NZ 4801: 2000. Strathfield, NSW: Author.

Stephan, S. (2001). Improving the safety culture of the Australian mining industry. J. Occup. Health and Safety - Aust. NZ, 17(3), 237-249.

St George, U., Lingard, P., \& James, W. (1999). A model intervention program for the management of workplace chemical safety. J. Occup. Health Safety - Aust. NZ, 15(2), 137-140. 
Strobach, H. (1990, June). Safety, a way of life. Safety Management, 41-42.

Sweeney, J.C. (1992). Measuring process safety managernent. Plant/Operations Progress, 11(2). 89-98.

Sweeney, S. (1994). Opportunities/strategies and tactics for going beyond lost time injuries. In OHS Positive Performance Indicators Part 1, Sydney, NSW: Worksafe Australia.

Sytsma, S. \& Manley, K. (1999). The quality tools cookbook. Retrieved January 13, 2003 from

http://www.sytsma.com/tqmtools/tqmtoolmenu.html

Telecom (1994). Quality in Australia: The Telecom archetype study, Melbourne, Victoria: Telstra Corporation.

Toca, F.M., \& Woodhull, D. (1996). Management of people and programs in Industrial Hygiene. Fairfax: American Industrial Hygiene Association.

Toohey, J. (1987). Managing health and safety effectively? Or is it all a bit ...technical? J. Occup. Health and Safety - Aust. NZ, 3(3), 235-239.

Topf, M. (1997, October). Beyond behaviour: reaching employees where it matters. Professional Safety, 30-31.

Trethewy, R., Gardner, D., Cross, J. \& Marosszeky, M. (2001).

Behavioural safety and incentive schemes. J. Occup. Health and Safety - Aust. NZ, 17(3), 251-262.

Tucker, F.G., Zivan, S.M., \& Camp, R.C. (1987, January/February). How to measure yourself against the best. Harvard Business Review, 8-10.

Tye, J. \& Brown, V. (1990, June). Insurance vs safety. Safety Management. 24-29.

Vecchio-Sadus, A.M. (2000, June). OHSE on the world wide web. Chemistry in Australia, 14-17. 
Vincoli, J. (1991, June). Total Quality Management and the safety and health professional. Professional Safety, 1991, 27-32.

Wallace, I. (1991, March). Evaluating safety performance. The Health and Safety Practitioner, 16-19.

Walleck, S.A., O'Halioran, J.D., \& Leader,C.A. (1991). Benchmarking world-class performance. The McKinsey Quartenly, Number 1, 324.

Waring, A. (1991, September). Success with safety management systems. The Safety \& Health Practitioner, 20-23.

Waterhouse, P. (1992, August). Safety audits: a company under the microscope. Health and Safety at Work, 15-16.

Watson, G.H. (1992). The benchmarking workbook - adapting best practices for performance improvement. Cambridge: Productivity Press.

Webster, B. (2000, September). Surveying people - designing questionnaires. Research Contract, 12(3), Edith Cowan University.

Wiarda, E.A., \& Luria, D.D. (1997). The "best practice" company and other benchmarking myths: Five lessons from real data. Retrieved July 22, 1998 from http://www.benchnet.com/bestpracticeco.htm

Williams, R. (1993). Measuring and assessing the effectiveness of health and safety programs. In Papers from Minesafe International 1993 (pp. 55-68). Perth, Western Australia: Chamber of Minerals and Energy.

Wilson, H. (1999, September). Extending the influence of occupational hygiene. American Academy of Industrial Hygiene Newsletter, Issue 99-3, 3.

Winder, C. (1995, September). Best practice in workplace hazardous substances management. Quality assurance: Best Practice, Regulation and Law, 4(3), 211-225. 
Winder, C. (1997). Integrating quality, safety and environmental management systems. Quality Assurance, 5, 27-48.

Winder, C. (1999a). The long road to the hazardous substances regulation. J. Occup. Health and Safety-Aust. NZ, 15(2), 99-102.

Winder, C. (1999b). Applying risk management approaches to chemical hazards. J. Occup. Health and Safety - Aust. NZ, 15(2), 161-169.

Winder, C., Gardner, D., \& Trethewy, R. (2001). Occupational health and safety management systems: recent Australian developments, J. Occup. Health and Safety - Aust. NZ, 17(1), 67-77.

Wooden, M., \& Vandenheuvel, A. (1999). Is occupational health and safety good for business? J. Occup. Health and Safety - Aust. NZ, 15(5), 411-416.

Worksafe Australia. (1993). Ergonomics for the control of strains and sprains in mining. Sydney, NSW: Author.

Worksafe Australia. (1995). OHS - good for business. Canberra, ACT: AGPS.

Worksafe Western Australia. (1995). Safety and health representatives handbook. Perth, Western Australia: Author.

Worksafe Western Australia. (1996). Occupational safety and health regulations 1996, part 5 - Hazardous substances - divisions 1 and 2. Retrieved June 29, 1999 from http://www.safetyline.wa.gov.au/pagebin/regohsr0027.asp

Worksafe Western Australia Commission. (2000). Code of practice: Manual handling. Perth: Author.

World Health Organisation. (1998). The international programme of chemical safety: Fact sheet No. 87. Retrieved August 10, 2000 from http://www.who.int/inf-fs/en/fact087.html 
Wyatt, A. (Ed.) (1995). Workplace health and safety manual. ( $2^{\text {nd }}$ rev. ed.). Sydney: $\mathrm{CCH}$ International.

Young, R.B., \& Prud'homme, S.H. (1991, March). Building the foundation for a quality culture. Journal for Quality and Participation, 92-98.

Yousiph, M., \& Winder, C. (1999). Chemicals? But we've used chemicals for years! J. Occup. Health Safety - Aust. NZ, 15(2), 14. 


\section{APPENDIX 1}

THE SCOPE AND FUNCTIONS OF INDUSTRIAL HYGIENE 


\section{THE SCOPE AND FUNCTIONS OF INDUSTRIAL HYGIENE}

The following is a modified statement of the scope and functions of trie profession as described by the American Board of Industrial Hygiene (1984):

\section{Scope of Industrial Hygiene}

Industrial Hygiene primarily involves:

- The recognition of environmental factors and stresses associated with work and work operations, and the understanding of their effects on the individual and his/her well-being in the workplace and in the community;

- The evaluation, through training and experience, and with the aid of quantitative measurement techniques, of the magnitude of these factors and stresses in terms of ability to impair the individual's health and wellbeing; and

- The prescription of methods to control or reduce such factors and stresses when necessary to alleviate their effects.

\section{The Industrial Hygienist}

An Industrial Hygienist is a person having a college or university degree or degrees in engineering, chemistry, physics, medicine or related biological sciences, who by virtue of special studies and training, has acquired competence in Industrial Hygiene.

\section{Functions of the Industrial Hygienist}

Within his/her sphere of responsibility the Industrial Hygienist will:

1. Direct the Industrial Hygiene program.

2. Examine the work environment and environs. 
- Study work operations and processes and obtain full details of the nature of work, materials and equipment used, products and by-products, number and sex of employees, and hours of work.

- Make appropriate measurements to determine the magnitude of exposure or nuisance to ivorkers and the public. In so doing he/she will:

- Select or devise methods and instruments suitable for such measurements;

- Personally, or through others under his/her direct supervision, conduct such measurements; and

- Study and test material associated with the work operations.

- Study and test biological materials, such as blood and urine, by chemical and physical means, when such examinations will aid in determining the extent and nature of exposure.

3. Interpret results of examination of the work environment and environs in terms of the ability to impair health, the nature of any health impairment, employee efficiency and community nuisance and/or damage, and present specific conclusions to appropriate interested parties such as management and health officials.

4. Make specific decisions as to the need for, or effectiveness of, control measures, and when necessary advise as to the procedures which will be suitable and effective for both the workplace and its surroundings.

5. Prepare rules, regulations, standards and procedures for the healthful conduct of work and prevention of nuisance in the community.

6. Present expert testimony before courts of law, hearing boards, worker's compensation commissions, regulatory agencies and legaliyappointed investigative bodies covering all matters pertaining to Industrial Hygiene as described in this document.

7. Prepare appropriate text for labels and precautionary information for materials and products to be used by employees and the public. 
8. Conduct programs for education of employees and the public in the prevention of occupational disease and community nuisance.

9. Conduct epidemiological studies of employee groups and industries to determine to what extent occupational disease is present, and to establish or improve threshold limit values or standards as guides for the maintenance of health and efficiency.

10. Conduct research to advance knowledge concerning the effects of occupation upon health and means of preventing occupational health impairment, community air pollution, noise, nuisance, and related problems.

Although not specifically mentioned in the official statement of the American Industrial Hygiene Association, the American Board of Industrial Hygiene considers that concern for health and well being of the consumers of the products of industry is implied by the words "arising in or from the workplace". 


\section{APPENDIX 2}

\section{LEGISLATIVE FRAMEWORK IN WESTERN}

AUSTRALIA 


\section{LEGISLATIVE FRAMEWORK}

The Mines Safety and Inspection Act 1994 sets objectives to promote and improve OHS standards. The Act sets out broad duties and is supported by more detailed requirements in the Mines Safety and Inspection Regulations 1995. A range of guidance material, including Guidelines, further supports the legislation. The legislative framework is set out in Figure 40 below.

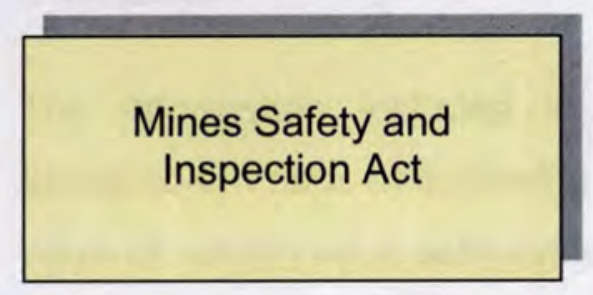

Major Provisions:

- The General Duties

- Management of Mines

- Enforcement of Act and Regulations

- Resolution of issues

- Safety and Heath Representatives

- Safety and Health Committees

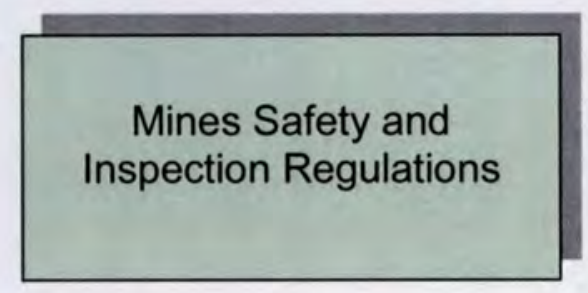

The Mines Safety and Inspection Regulations set minimum requirements for specific hazards and work practices, including reference to National Standards developed by the National Occupational Health and Safety Commission and Australian Standards developed by Standards Australia.

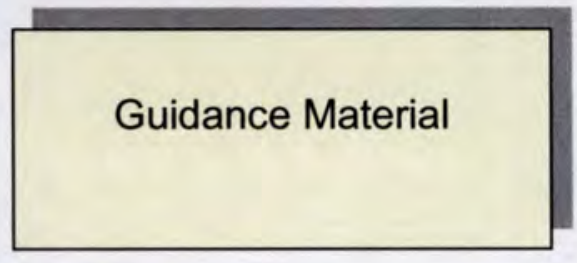

- Codes of Practice approved for WA mines in accordance with Section 93 of the Act.

- Guidelines produced by the Mines Occupational Safety and Health Advisory Board (MOSHAB).

- National Codes of Practice and National Standards developed by the National Occupational Health and Safety Commission.

- Australian Standards developed by Standards Australia

Figure 40: Legislative framework

(Mines Occupational Safety and Health Advisory Board, 1999, p.13) 
Guidance material includes explanatory documents that provide more detailed information on the requirements of the legislation and include codes of practice and guidelines.

Guidelines contain practical information on how to comply with legislative requirements. They describe safe work practices that can be used to reduce the risk of work-related injury and disease and may also contain explanatory information.

The information included in a Guideline may not represent the only acceptable means of achieving the standard referred to. There may be other ways of setting up a safe system of work and, providing the risk of injury or disease is reduced as far as practicable, the alternatives should be acceptable. 


\section{APPENDIX 3}

\section{PERFORMANCE REPORTING AND REVIEW}

- The OHS Dashboard

- The Safety Performance Index

- The Balanced Scorecard 


\section{PERFORMANCE REPORTING AND REVIEW}

\section{- The OHS Dashboard}

The "dashboard" covers several critical aspects of OHS performance. The example given below describes progress at individual sites, together with the broader performance of Alcoa's Operations in Western Australia. One element of the dashboard, that dealing with behavioural safety programs, is represented below. This provides data on activity levels attached to task observations and to management safety contacts, as described earlier.

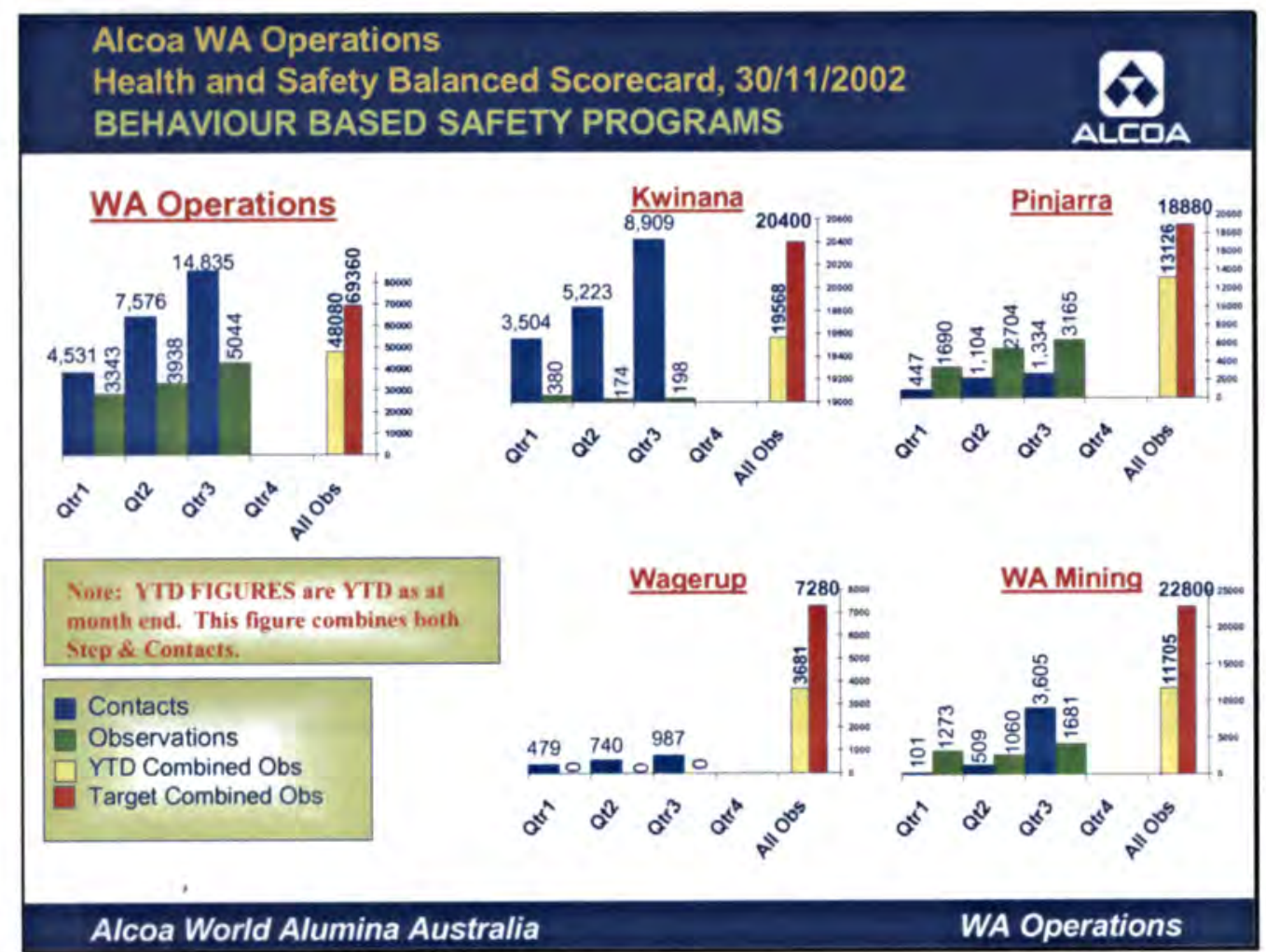

(Alcoa, 2002b, p. 12) 


\section{PERFORMANCE REPORTING AND REVIEW}

\section{- The Safety Performance Index}

The Index applies a rating and a score to selected elements of the Company's health and safety program. A bottom line figure is used to represent overall performance for the month.

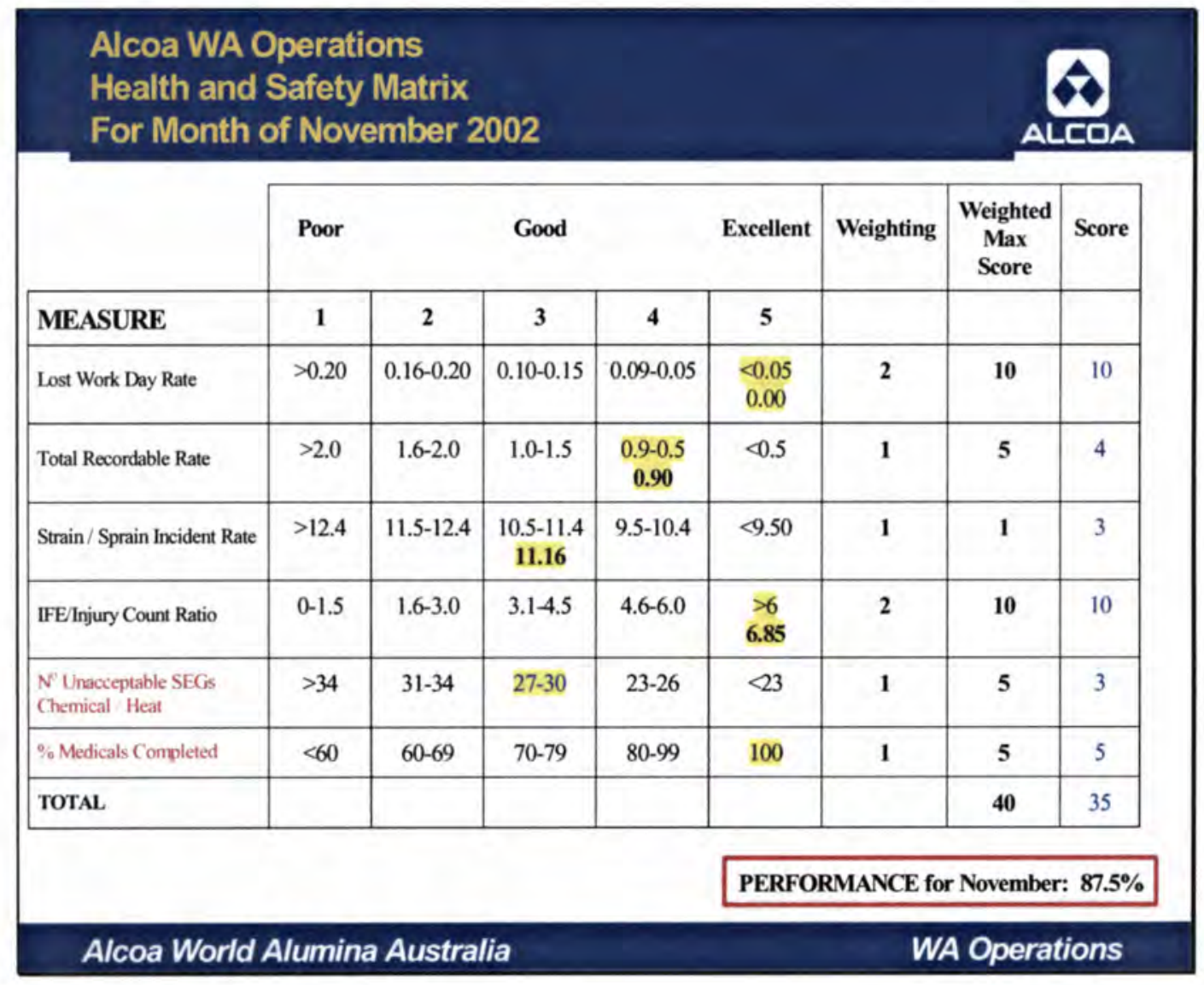

(Alcoa, 2002b, p. 15) 


\section{PERFORMANCE REPORTING AND REVIEW}

\section{- The Balanced Scorecard}

The Balanced Scorecard is applied to various key elements of facility management. A typical "A3" has a section dealing with the business case (for action), followed by statements dealing with the present condition, the future (desired) condition and an action plan to achieve the outcomes that are wanted. In the following example, the names of individuals nominated for Single Point Accountability (SPA) purposes have been deleted.

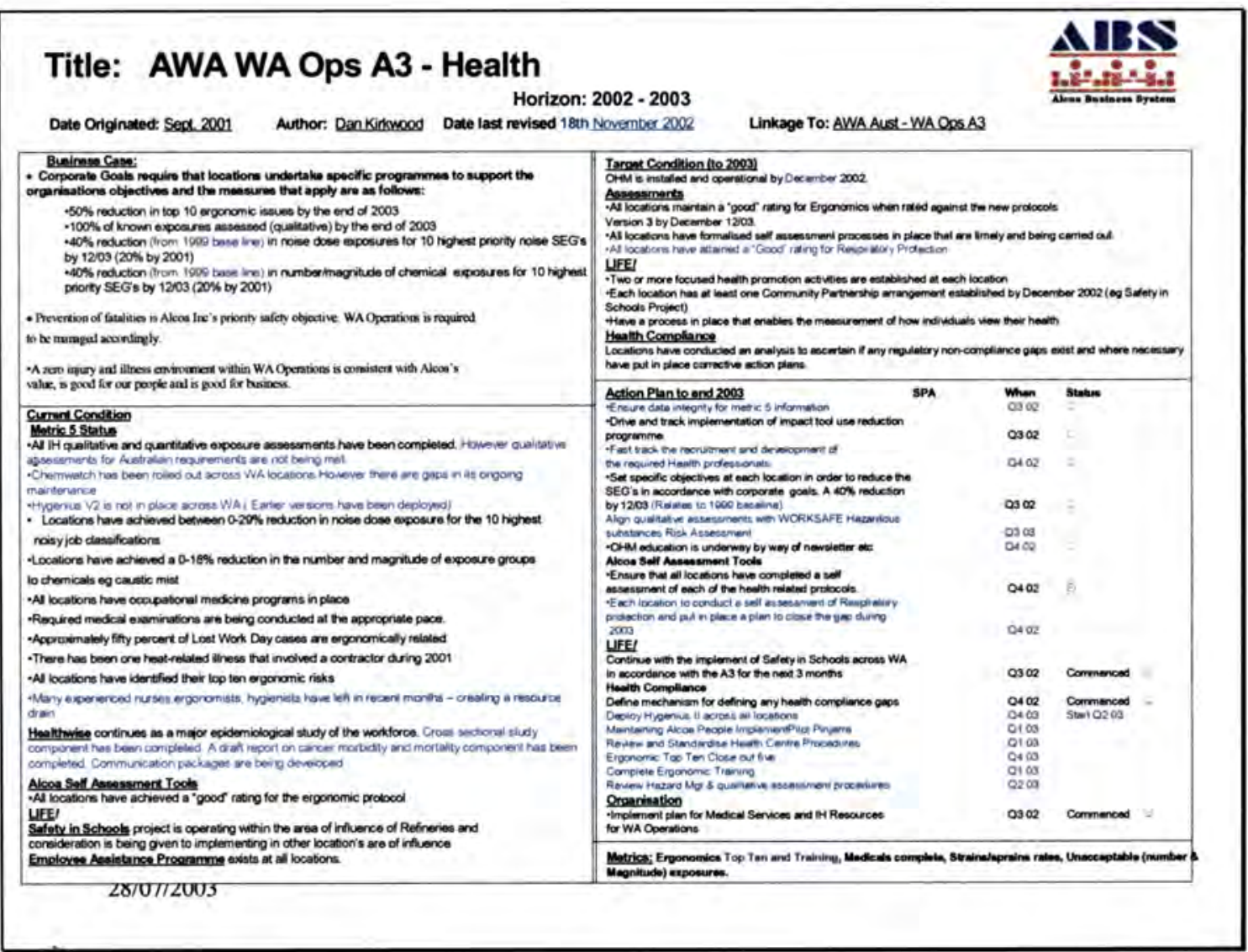

(Alcoa, 2002b, p. 18) 


\section{APPENDIX 4}

\section{POWERPOINT PRESENTATION PACKAGE \\ - STUDY PROPOSAL}




\section{Benchmarking Study : Chemical \\ Safety}

In Pursuit of Best Practice: Benchmarking Tools and Processes for the Management of

Hazardous Substances in the Workplace

\section{Benchmarking}

"Fools you are to say you learn by your experience ... I prefer to profit by others ' mistakes and avoid the price of my own."

\section{Benchmarking - A Few Views}

- "Benchmarking involves continually researching for new ideas and methods, processes and practices, and either adopting or adapting the best features of these.

- "It is important to not only identify what is best, but also to understand how best is achieved."

- "Benchmarking is about building up relationships and communicating ideas between enterprises. "

- "Benchmarking is an important process for fast tracking improvement activities. 


\section{Purpose of the Study}

- To develop effective tools and processes for benchmarking hazardous materials management practices.

- To gauge the effectiveness of these tools and processes as agents of change.

- To assess the transferability of the tools and processes to other critical elements of OHS management.

\section{Significance of the Study}

- Hazardous materials are ubiquitous, often poorly understood and feature in many workplace accidents

- The chemical safety area is very complex,with many regulations, codes and guidance materials.

- Benchmarking practices around the world tend to be either of a simple, unstructured kind, or very complex and formal - requiring extensive commitment of time and resources. There is little in-between.

- There is a paucity of simple, flexible and effective benchmarking tools.

\section{Significance of the Study (cont.)}

- There is potential for the materials and processes developed in this study to be used widely in the transfer of best practice. The materials account for the organisation of work factor, the workplace environment factor and the people factor.

- Study materials will assist with the selection of benchmarking partners and will help to identify "pockets of excellence" for focused attention. 


\section{Conceptual Framework}

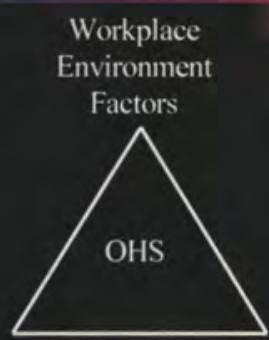

\section{Sources of Information}

Literature dealing with:

- Quality management \& continuous improvement

- Management systems, organisational effectiveness \& change management

- Behavioural safety

- Risk management

- Performance measurement, reporting \& review

- Compliance, standards \& auditing

- Benchmarking, best practice \& technology transfer

\section{Scope of the Study}

- The study is dealing with hazardous materials generally ie. it is not addressing specific substances or groups of substances.

- The study has an occupational focus ie. it does not seek to address environmental or community issues or impacts.

- The study does not deal with the explosives and dangerous goods area. 


\section{Research Questions}

Is there a suite of practical benchmarking tools and methodologies capable of:

- Accounting for the people factor, the workplace environment factor and the organisation of work factor - as they relate to chemical safety?

- Application to both medium and large organisations?

- Application to other elements under the OHS umbrella ie. to areas other than chemical safety?

- Identifying pockets of excellence?

- Facilitating the transfer of best practice in chemical safety?

\section{Study Design}

- The study is focused on eight facilities within the WA Mining Industry. These are a mix of medium and large sites - some are mine sites, others are mineral processing sites.

- Sites are broadly representative of the Industry ie. drawn from the bauxite, alumina, gold, nickel and iron ore sectors.

\section{Study Design (cont.)}

\section{The study is built on three assumptions:}

- That the site manager and OHS professional will be aware of any innovations or exceptional practice at the site.

- That the structured sequence of booklets will flush out "pockets of excellence" for use in a two-way exchange between benchmarking partners.

- That almost all sites will have something to offer. 


\section{Data Gathering, Review \&}

\section{Reporting}

This element will pursued via a set of eight booklets, viz:

- Book 1: Site Profile

- Book 2: Preliminary Questions

- Book 3: Compliance

- Book 4: Organisation of Work

- Book 5: The Workplace Environment

- Book 6: People Initiatives

- Book 7: Results - site strengths and opportunities

- Book 8: Scoresheets, comparisons \& benchmarking opportunities

\section{Some Areas Covered}

Preliminary (Booklets 1 \& 2)

- Organisational details

- Yes/no questionnaires on OHS (general) - on elements such as policy, planning, roles \& responsibilities, involvement, measurement, technology transfer, self ranking, etc.

- Yes/no questionnaires on chemical safety - on elements such as inventories, MSDS deployment, labelling, screening of new chemicals, use, compliance, contractor materials, critical success factors, etc.

\section{Some Areas Covered (Cont.)}

Main (Booklets 3, 4, 5 \& 6)

- Yes/no questionnaires on compliance.

- Rating questions on organisation of work (leadership, commitment, planning, organisation, training, communication and measurement).

- Rating questions on the workplace environment (hazard identification, risk assessment \& risk control).

- Rating questions on people initiatives (behavioural safety, motivation, morale, promotional efforts, etc). 


\section{A Typical Rating Question)}

Please rate the use of leading indicators of OHS performance on-site.

\begin{tabular}{|l|l|l|l|}
\hline Poor & Fair & Good & Excellent \\
\hline
\end{tabular}

If "Good" or "Excellent", what are the key initiatives or success factors in your approach?

-

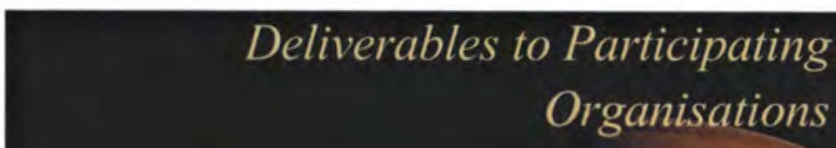

- A detailed review of management systems and procedures in relation to chemical safety.

- An appraisal of the site's level of compliance with statutory requirements for chemical safety.

- Reports on status of programs, improvement opportunities, pockets of excellence and performance scores in regard to organisation of work, the workplace environment and people factors.

- Summary information on state-of-the-art thinking with respect to effective management of hazardous materials.

- Access to a suite of benchmarking tools and processes generated by the study. 


\section{APPENDIX 5}

\section{APPROVAL REQUEST LETTERS - SITE VISITS}

(SAMPLE) 
Mr John Smith,

OHS Superintendent,

Sparkles Mine,

Leonora,

WA

Date

Dear John,

Re: Development of Benchmarkiıg Toois and Processes - Hazardous Materials Management

Further to our recent telephone conversation, I would like to confirm arrangements for me to malie a preliminary visit to your site next month.

I have attached an abstract of the PhD study proposal, as submitted to Edith Cowan University. This overviews the improvement opportunity, the methods of data collection, expected study outcomes and the broad benefits attached to an advance with benchmarking tools and processes as they apply to the management of hazardous materials.

More specifically, for participating locations such as yours, there will be a number of benefits. Your site will receive a detailed review of the status of its management systems and procedures in relation to hazardous materials. You will receive an appraisal of the site's level of compliance with statutory requirements and a series of reports that identify the status of programs, improvement opportunities, pockets of excellence and performance scores in regard to management enablers, technical initiatives and people initiatives. Additionally, you will receive summary information on state-of-the-art thinking with respect to effective management of hazardous materials (and OHS). Furthermore, your organisation will have access to a suite of benchmarking tools and processes generated by the study. Hopefully, these will assist you to select future benchmarking partners and to identify "pockets of excellence" for focused attention.

All data gathered at your site will be shared with you as it is generated, as will aggregated material and broad study outcomes. Site data will be subject to strict safeguards to protect privacy and confidentiality. Study results will be presented in a way that ensures anonymity for participating locations and data will be secure in a locked filing cabinet in my office at Kwinana. There will be no third party access to the information.

Please confirm that these proposals are satisfactory to you. As discussed previously, I will forward a package of materials for you to complete in advance of the visit. This will make our time together more productive. 
Please call me on (08) 94103301 if you have any queries or concerns in the meantime. Thank you again for you support of this project. I look forward to meeting with you next month.

Best wishes,

\section{Barry Chesson}

Manager - Occupational Hygiene

Alcoa World Alumina Australia 
Mr Bill Brown,

Registered Mine Manager,

Sparkles Mine,

Leonora,

WA

Date

Dear Bill,

Re: Development of Benchmarking Tools and Processes Hazardous Materials Management

Further to our recent telephone conversation, I would like to confirm our arrangements for my visit to your site next month.

I have attached an abstract of the PhD study proposal, as submitted to Edith Cowan University. This overviews the improvement opportunity, the methods of data collection, expected study outcomes and the broad benefits attached to an advance with benchmarking tools and processes as they apply to the management of hazardous materials.

More specifically, for participating locations such as yours, there will be a number of benefits and these will be made available on a complimentary basis. Your site will receive a detailed review of the status of its management systems and procedures in relation to chemical safety. You will receive an appraisal of the site's level of compliance with statutory requirements and a series of reports that identify the status of programs, improvement opportunities, pockets of excellence and performance scores in regard to organisation of work (management enablers), the workplace environment (technical initiatives) and people initiatives. Additionally, you will receive summary information from an extensive literature review on state-of-the-art thinking with respect to effective management of hazardous materials (and OHS). Furthermore, your organisation will have access to a suite of benchmarking tools and processes generated by the study. Hopefully, these will assist you to select future benchmarking partners and to identify "pockets of excellence" for focused attention.

All data gathered at your site will be shared with you as it is generated, as will aggregated material and broad study outcomes. Site data will be subject to strict safeguards to protect privacy and corifidentiality. Study results will be presented in a way that ensures anonymity for participating locations and data will be secure in a locked filing cabinet in my office at Kwinana. There will be no third party access to the information. The exercise involves 8 locations within the WA Mining and Mineral Processing Industry. Some comparisons will be made in order 
to identify drivers and characteristics that impact in a significant way on OHS outcomes. Again, every effort will be made to ensure that a reader of the thesis or any subsequent papers would not be able to identify particular sites.

The University requires that the interests of organisations and individuals participating in research are protected at all times. Accordingly, would you please indicate via the attached sheet if the above proposals are satisfactory to your organisation. Also, please indicate if you would be comfortable with data generated at your site being used in this and any further research or if you would prefer it to be used for the present study only. As discussed previously, I will forward a package of materials for you to complete in advance of the visit. This will make our time together more productive.

Please call me on (08) 94103301 if you have any queries or concerns in the meantime. Thank you again for you support of this project. I look forward to meeting with you next month.

Best wishes,

Barry Chesson

Manager - Occupational Hygiene

Alcoa World Alumina Australia 


\section{Consent Form}

Re: PhD Project (Edith Cowan University) "Development of Benchmarking Tools and Processes " Chemical Safety"

I am familiar with the proposals attached to the above captioned study and explained by Barry Chesson, and confirm that consent is given for the conduct of research activities at this site.

With regard to materials collected or developed as a result of field-work at this site:

Approval is given for data generated in this study to be used for the present research and for any subsequent research that might arise.

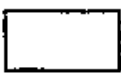

Or

Approval is given for data generated in this study to be used for the present research only.

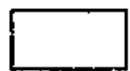

(Please tick appropriate box)

Signed:

Position:

Facility:

Date: 
APPENDIX 6

SAMPLE PROGRAM FOR SITE VISITS 
SAMPLE PROGRAM FOR SITE VISITS

\begin{tabular}{|c|c|c|}
\hline Element & Activity & $\begin{array}{l}\text { Researcher } \\
\text { interaction with: }\end{array}$ \\
\hline 1. & $\begin{array}{l}\text { Initial discussions with Site } \\
\text { Management: } \\
\text { - Outline of aims of the study and } \\
\text { deliverables for participating } \\
\text { sites (to reinforce earlier } \\
\text { communications). } \\
\text { - Review of Management's } \\
\text { philosophy and approach to } \\
\text { OHS on the site } \\
\text { - Review of planned activities at } \\
\text { the site. }\end{array}$ & $\begin{array}{l}\text { Site Manager and } \\
\text { OHS Professional }\end{array}$ \\
\hline 2. & $\begin{array}{l}\text { Review of material supplied by the site } \\
\text { professional contact (via completion of } \\
\text { Booklets } 1 \& 2 \text { ). }\end{array}$ & $\begin{array}{l}\text { Site OHS } \\
\text { Professional }\end{array}$ \\
\hline 3. & $\begin{array}{l}\text { Detailed discussions on OHS and } \\
\text { chemical safety approaches, tools and } \\
\text { initiatives at the site - facilitated by } \\
\text { working through Booklets 3-6. }\end{array}$ & $\begin{array}{l}\text { Site OHS } \\
\text { Professional }\end{array}$ \\
\hline 4. & $\begin{array}{l}\text { Verification activities: } \\
\text { - Site tour } \\
\text { - Review of documentation (hard } \\
\text { copy and electronic). }\end{array}$ & $\begin{array}{l}\text { Site OHS } \\
\text { Professional }\end{array}$ \\
\hline 5. & $\begin{array}{l}\text { Wrap-up session. Feedback on: } \\
\text { - Activities completed thus far. } \\
\text { - Initial impressions about OHS } \\
\text { and chemical safety on-site. } \\
\text { - Next steps. }\end{array}$ & $\begin{array}{l}\text { Site Manager and } \\
\text { OHS Professional }\end{array}$ \\
\hline
\end{tabular}




\section{APPENDIX 7}

\section{DATA COLLECTION, ANALYSIS AND COMMUNICATION}

- Booklet 1: Site Profile

- Booklet 2: Preliminary Questions

- Booklet 3: Compliance

- Booklet 4: Organisation of Work

- Booklet 5: The Workplace Environment

- Booklet 6: People Initiatives

- Booklet 7: Results - Site Strengths and Opportunities for Improvement

- Booklet 8: Scoresheets, Inter-site Comparisons and Benchmarking Opportunities 

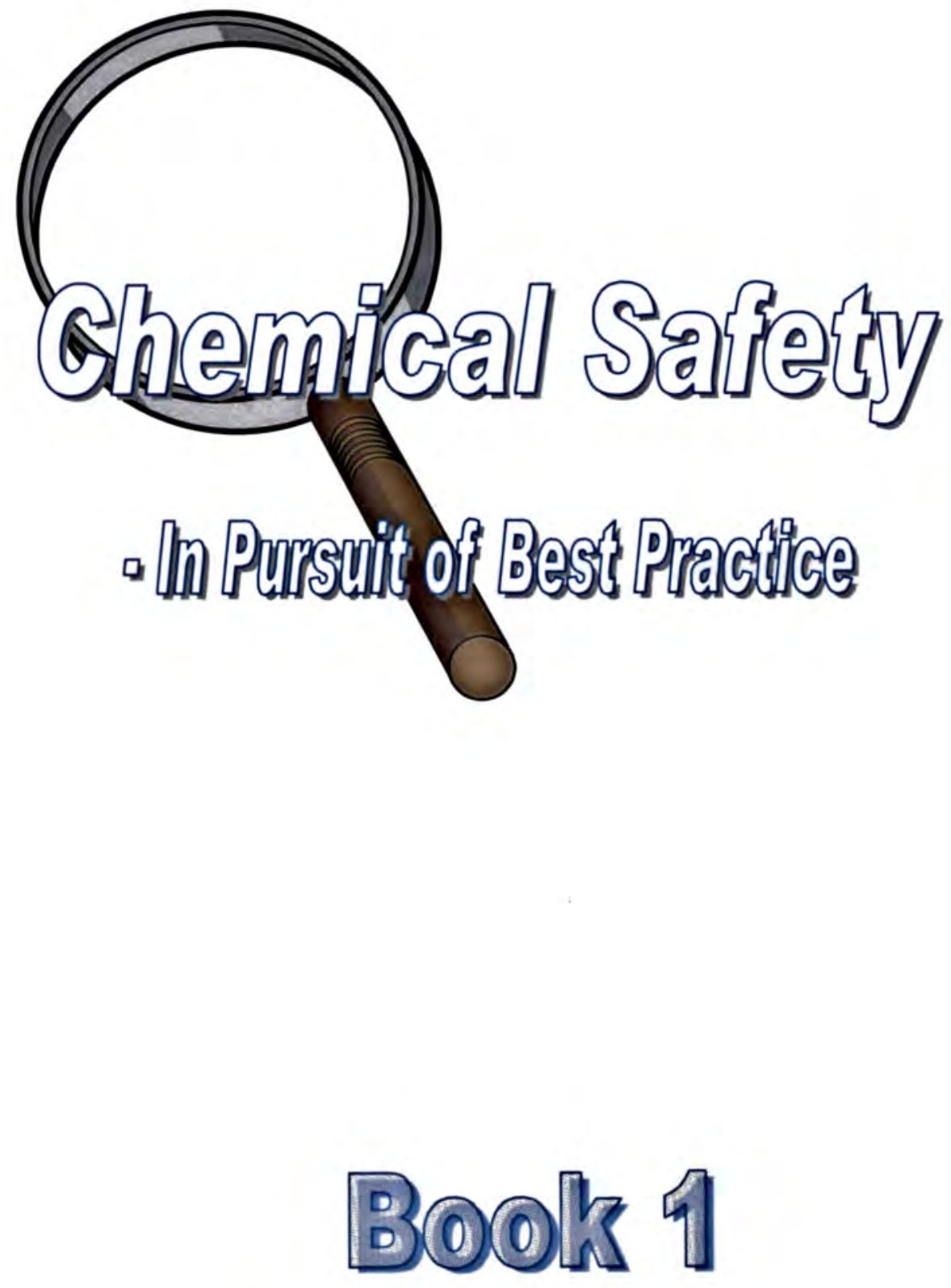

- Silte Profille -

Barry Chesson

September 2002 
Book 1 is designed to gather basic information on the nature of work at the facility, arrangements for dealing with $\mathrm{OHS}$ and principal OHS issues and challenges. Preliminary data gathered in this way will be studied by the Researcher and utilised to focus the inquiry and make site visits as productive as possible.

SITE:

CONTACT PERSON:

1. THE ENTERPRISE

Name of Organisation:

Division/Plant:

Address:

Function of Plant:

Contact Person:

Job Title:

Phone No:

E-mail:

\section{THE OPERATION}

Main raw materials:

Main end product:

Main waste materials:

General description of process, plant, equipment and activities: 
3. THE WORKFORCE

Numbers:

Employees (total): Management/admin:

Production: Maintenance:

Shift Fattern:

Contractor arrangements and numbers:

4. ORGANISATION FOR OHS

5. PRINCIPAL OHS ISSUES \& CHALLENGES

Physical hazards:

Chemical hazards:

Biological hazards:

Ergonomic hazards:

6. OTHER RELEVANT INFORMATION

Attachments: Please attach plant map(s), organisation charts, process descriptions, further sheets as may be needed to respond to the above, or other items relevant to the site visit. 
* This research forms part of PhD studies being undertaken by Barry Chesson, Manager - Occupational Hygiene, Alcoa World Alumina Australia. The studies are being pursued though the Faculty of Communications, Health and Science at Edith Cowan University (Joondalup Campus).

* The full study title is "In Pursuit of Best Practice: Benchmarking Tools and Processes for the Management of Hazardous Substances in the Workplace."

* This study is seeking to develop and test new tools and processes to make benchmarking activity and the transfer of technology, ideas and approaches more efficient and meaningful. it will focus on the management of chemicals within the Western Australian Mining Industry, but outcomes should be applicable within the wider framework of OHS and to other industry sectors.

* It will draw heavily from state-of-the-art safety management theory and will seek to establish the linkage between the organisation of work factor, the workplace environment factor and the people factor as they contribute to workplace health and safety performance.

* Six booklets will be used to assist with the gathering of data for this project. A further two booklets will be used for summarising results and providing feedback to participating sites. The booklets are:

Bock 1 Site Profile

Book 2 Preliminary Questions

Book 3 Compliance

Book 4 Organisation of Work

Book 5 The Workplace Environment

Book 6 People

Book 7 Results - Site strengths and opportunities for improvement

Book 8 Scoresheets, inter-site comparisons and benchmarking opportunities

Study outcomes will assist with the selection of benchmarking partners and will help to identify "pockets of excellence" for focused attention. This will encourage and assist organisations to take steps tcwards identifying and implementing Industry best practice in the OHS element of interest.

- For further information please contact the researcher, Barry Chesson, on (08) $94103301(W)$ or $\quad$ or on e-mail at barry.chesson@alcoa:com.au.

(C) 


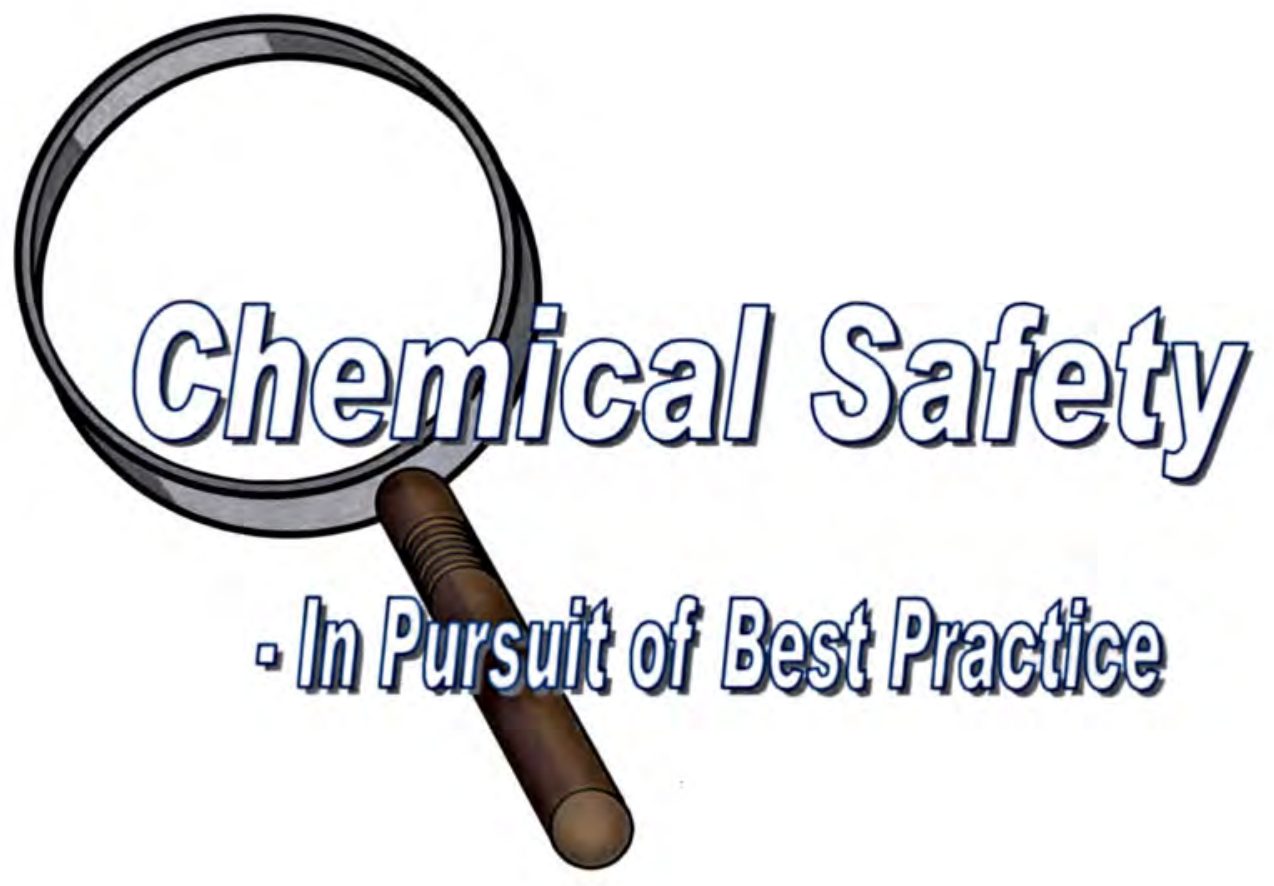

Book?

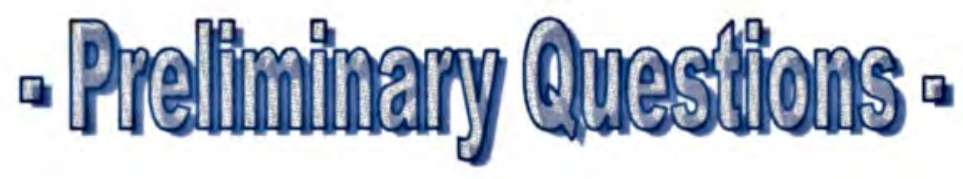

Barry Chesson

September 2002 
Book 2 is designed to gather initial information on the way $\mathrm{OHS}$, and chemical safety in particular, is managed on the site. Preliminary data gathered in this way will be studied by the Researcher and utilised to focus the inquiry and make site visits as productive as possible.

SITE:

CONTACT PERSON:

PART A: GENERAL OHS

\section{A1 Yes/No Questionnaire}

Does your facility have:

Yes No

1. A clearly expressed OHS policy?

2. A philosophy and commitment to exceed minimum standards?

3. Integration of OHS into the key business functions of planning, organising, leading, controlling and evaluating?

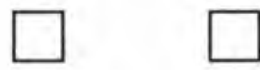

4. Clearly defined OHS performance expectations and measures for all levels in the organisation?

5. Injury management plans?

6. Clear evidence of management involvement and commitment to OHS?

7. Clearly assigned OHS responsibilities for managers and supervisors, expressed in terms of their normal duties?

8. Commitment and involvement of employees, with formal and informal structures 
to encourage their participation?

9. A generally held view within Management that

Corporate investment in OHS is good business practice?

A2 Benchmarking, Best Practice and Technology Transfer

1. Has your site been involved in a formal OHS benchmarking exercise?

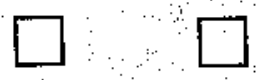

2. Has your site been inyolved in structured activities aimed at identifying best practice with respect to OHS?

3. Does your site take part in a formal OHS audit program?

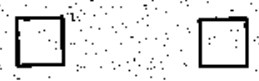

4. Has your site been involved in structured technology transfer activities for OHS?

A3 Measurement

1. What forms of performance measurement are applied to OHS at your site? Please give examples.

Lagging indicators?

Leading indicators? 


\section{A4 Self Ranking in OHS}

Please indicate in which category you believe your site belongs, relative to the Mining Industry of WA:

Top 5 percent

Top 10 percent, but not top 5 percent

Top 25 percent, but not top 10 percent

Top half, but not top 25 percent

Top 75 percent, but not top half

Bottom 25 percent

\section{A5 Potential Benchmarking Partners}

What other organisations do you admire in terms of their performance or reputation in terms of management of OHS? 
B1 Yes/No Questionnare

1. Does your location have a published policy on the management of hazardous materials?

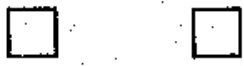

2. Have roles and responsibilities been clearly assigned in relation to the management of hazardous materials at the site?

3. Is there a current hazardous materials inventory for your location?

4. Do you have a material safety data sheet (MSDS), or equivalent, on-site for every hazardous material entering or leaving the site?

5. Does an MSDS normally accompany the first supply of a hazardous material to your location?
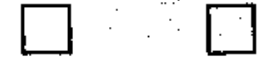

6. Do the purchasing and safety/health groups interact to screen new materials requested for purchase?

7. Is there a system to prevent non-approved chemicals from coming on-site and to ensure that those chemicals that are on-site have been approved?

8. Is there a system to track where and how much chemical usage occurs on-site?

9. Does your site comply with all relevant legal requirements for the safe storage, transport, issue, use and disposal of hazardous materials employed or generated by its operations? 
10. Do you label or post warning signs for every hazardous material used on-site or sent elsewhere from the site?

11. Are contractors expected to meet similar requirements/standards with respect to the hazardous materials they bring onto site?

\section{B2 Flow Chart}

Please provide a flow chart that describes how chemicals are managed at the site.

\section{B3 Critical Success Factors}

What do you consider to be the four most critical success factors in relation to chemical safety at your site?

1.

2.

3.

4. 
B4 Measurement

How do you measure performance in relation to these critical success factors?

B5 Innovations

What innovations (in the chemical safety area) have been introduced to your site? In other words, what do you do in this area that is different (and better) than other sites you have seen or heard about? 
* This research forms part of PhD studies being undertaken by Barry Chesson, Manager - Occupational Hygiene, Alcoa World Alumina Australia. The studies are being pursued though the Faculty of Communications, Health and Science at Edith Cowan University (Joondalup Campus).

* The full study titie is "In Pursuit of Best Practice: Benchmarking Tools and Processes for the Management of Hazardous Subsłances in the Workplace."

* This study is seeking to develop and test new tools and processes to make benchmarking activity and the transfer of technology, ideas and approaches more efficient and meaningful. It will focus on the management of chemicals within the Western Australian Mining Industry, but outcomes should be applicable within the wider framework of OHS and to other industry sectors.

* It will draw heavily from state-of-the-art safety management theory and will seek to establish the linkage between the organisation of work factor, the workplace environment factor and the people factor as they contribute to workplace heaith and safety performance.

* Six booklets will be used to assist with the gathering of data for this project. A further two booklets will be used for summarising results and providing feedback to participating sites. The booklets are as follows:

Book 1 Site Profile

Book 2 Preliminary Questions

Book 3 Compliance

Book 4 Organisation of Work

Book 5 The Workplace Environment

Book 6 People

Book 7 Results - Site strengths and opportunities for improvement

Book 8 Scoresheets, inter-site comparisons and benchmarking opportunities

Study outcomes will assist with the selection of benchmarking partners and will help to identify "pockets of excellence" for focused attention. This will encourage and assist organisations to take steps towards identifying and implementing Industry best practice in the OHS element of interest.

* For further information please contact the researcher, Barry Chesson, on (08) 94103301 (W) or $\quad$ or on e-mail at barry.chesson@alcoa.com.au.

(c) 


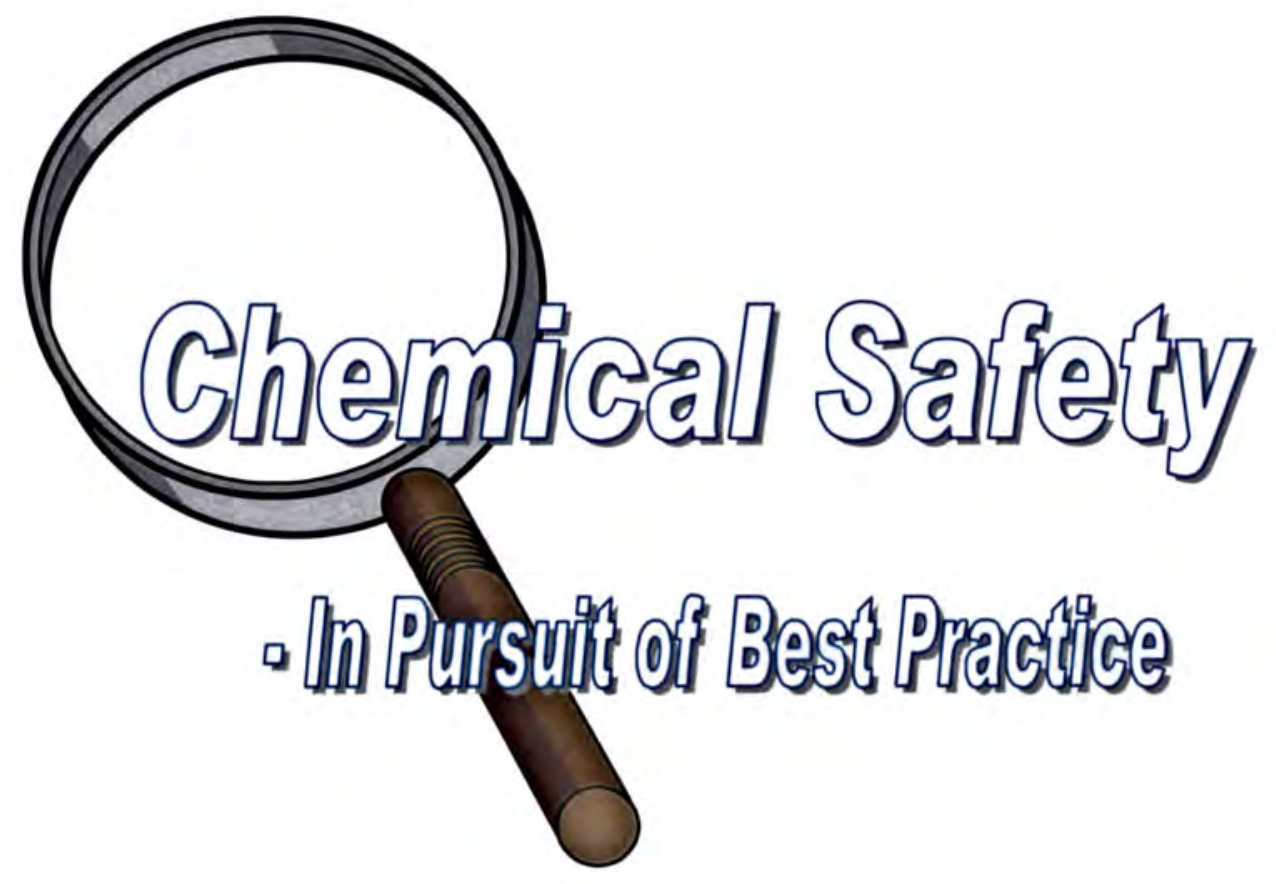

Bools 3

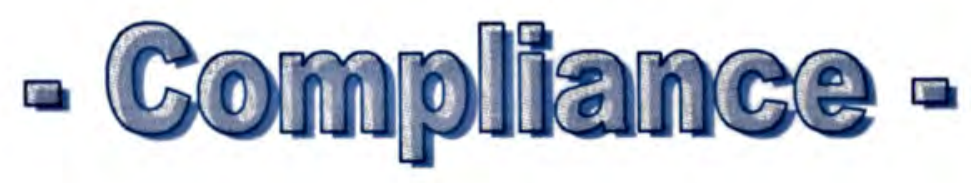

Barry Chesson

September 2002 
Book 3 is designed to stimulate discussion and gather information on how the site seeks to meet the chemical safety requirements expressed in the Mines Safety and Inspection Regulations 1995. The purpose is to identify innovative, cost effective and practical approaches to meeting the minimum requirements expressed in law

SITE:

CONTACT PERSON:

COMPLIANCE WITH THE MINES SAFETY \& INSPECTION REGULATIONS 1995

A. Material Safety Data Sheets (MSDSs)

(Provision of, where practicable)

Reg. 7.21

Y N N N $/ \mathbf{A}$

1. MSDSs suitable for the purpose

- Worksafe format?

- Current?

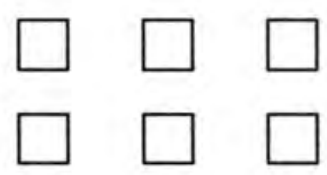

2. MSDSs available for contractor-supplied chemicals?

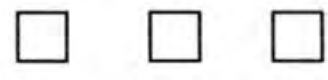

B. Containers

(Suitable design and manufacture)

Reg. 7.22

1. Chemical containers of suitable design and manufacture?

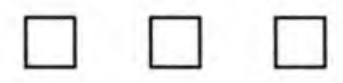

2. Transfer of bulk supplies to suitable, smaller containers? 
C. Disposal of Containers

(Safe means of disposal)

Reg. 7.23

1. Used containers returned to supplier?

2. Used containers cleaned and disposed of appropriately?

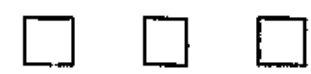

D. Labels

(Provision and care of labels)

1. Packaging labelled in accordance with ADG Code?

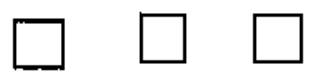

2. Labels applied when hazardous substances are transferred to small containers?

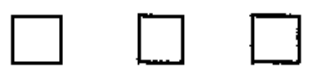

E. Registers

(Maintenance of register, inclusion of

Reg. 7.25

MSDSs, assessments and reports, accessibility)

1. MSDS register exists?

2. MSDS for each substance in the register? 
3. MSDS register freely accessible to employees?

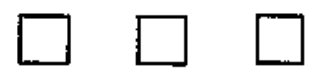

4. MSDS register details the duties that may give rise to exposure?

5. MSDS register contains details of any risk

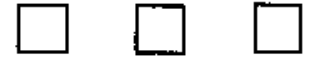

\title{
assessment made under Reg. 7.27?
}

\author{
(a)
} .

6. A mechanism is in place to regularly update MSDSs In the register?

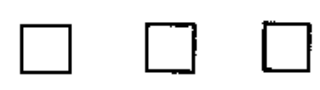


means by which risks can be reduced?

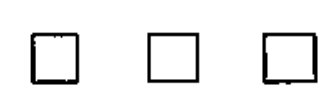

H. Risk reduction

(Using hierarchy of controls)

Reg. 7.28

1. Hierarchy of controls widely understood on-site?

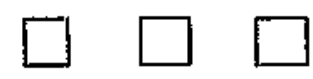

2. Hierarchy of controls well deployed?

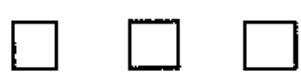

3. Procedures documented?

4. Suitable arrangements in place for PPE:

- selection in accordance with Aust. Stds?

- use in oxygen-deficient atmospheres?

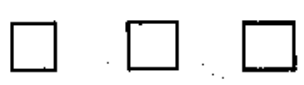

- where appropriate?

- availability?

- maintenance?

- training?

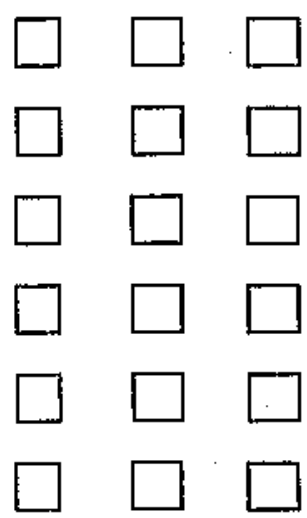

I. Atmospheric Monitoring

Reg. 7.29

(Sampling, recording and reporting in accordance with Part 9)

1. Sampling methodologies are in accordance With Australian Standards and Worksafe Australia Exposure Standards?

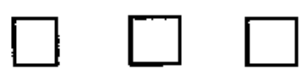


2. Employee exposure levels are below Worksafe Australia Exposure Standards?

J. Health Surveillance

(Provision of heaith surveillance

Reg. 7.30 where appropriate)

1. Health assessments in initial employment?

2. Health assessments on periodic basis?
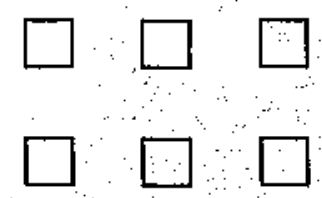

3. Health assessments based on specific occupational exposure or risk?

4. Biological monitoring carried out:

- on a routine basis?

- on an as-needed basis?

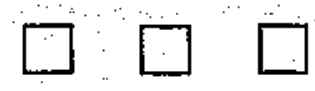

K. First Aid

(Provision of appropriate equipment,

Reg. 4.24 - 4.29 facilities and services)

1. Ready availability of resuscitation equipment?

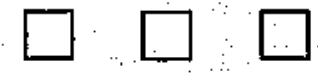

2. Availability of qualified first ald personnel when mine is working?

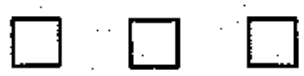


3. Above includes at least one person who is trained in use of resuscitation equipment?

4. First aid procedures are fully in place for:

- cyanide?

- corrosive substances?

- other very toxic materials?

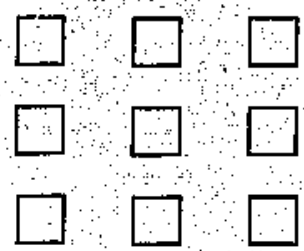

5. Safety showers appropriate in terms of:

- location?

- accessibility?

- signposting?

- maintenance?

- supply of cool, clean water?

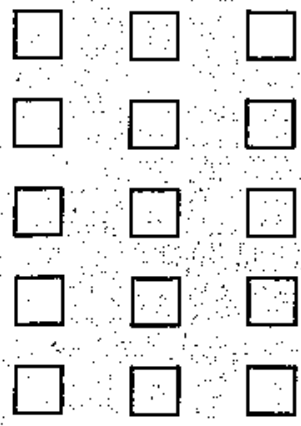

L. Other Matters

1. Records kept of induction and refresher training programs relating to chemical safety?

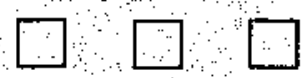

2. Adequate training in safety procedures and safe systems of work?

3. Employees trained in use of the MSDS?
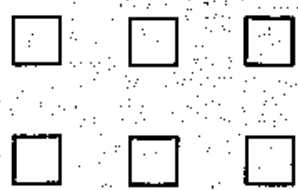
* This research forms part of PhD studies being undertaken by Barry Chesson, Manager - Occupational Hygiene, Alcoa World Alumina Australia. The studies are being pursued though the Faculty of Communications, Health and Science at Edith Cowan University (Joondalup Campus).

- The full study title is "In Pursuit of Best Practice: Benchmarking Tools and Processes for the Management of Hazardous Substances in the Workplace."

* This study is seeking to develop and test new tools and processes to make benchmarking activity and the transfer of technology, ideas and approaches more efficient and meaningful. It will focus on the management of chemicals within the Western Australian Mining Industry, but outcomes should be applicable within the wider framework of OHS and to other industry sectors.

* It will draw heavily from state-of-the-art management theory and will seek to establish the linkage between the people factor, the workplace environment factor and the organisation of work factor as they contribute to workplace health and safety performance.

* Six booklets will be used to assist with the gathering of data for this project. A further two booklets will be used for summarising results and providing feedback to participating sites. The booklets are as follows:
Book 1 Site Profile
Book 2 Preliminary Questions
Book 3 Compliance
Book 4 Organisation of Work
Book 5 The Workplace Environment
Book 6 People
Book 7 Results - Site strengths and opportunities for improvement
Book 8 Scoresheets, inter-site comparisons and benchmarking opportunities

* Study outcomes will assist with the selection of benchmarking partners and will help to identify "pockets of excellence" for focused attention. This will encourage and assist organisations to take steps towards identifying and implementing Industry best practice in the OHS element of interest.

* For further information please contact the researcher, Barry Chesson, on (08) $94103301(W)$ or $\quad$ or on e-mail at barry.chesson@alcoa.com.au.

() 


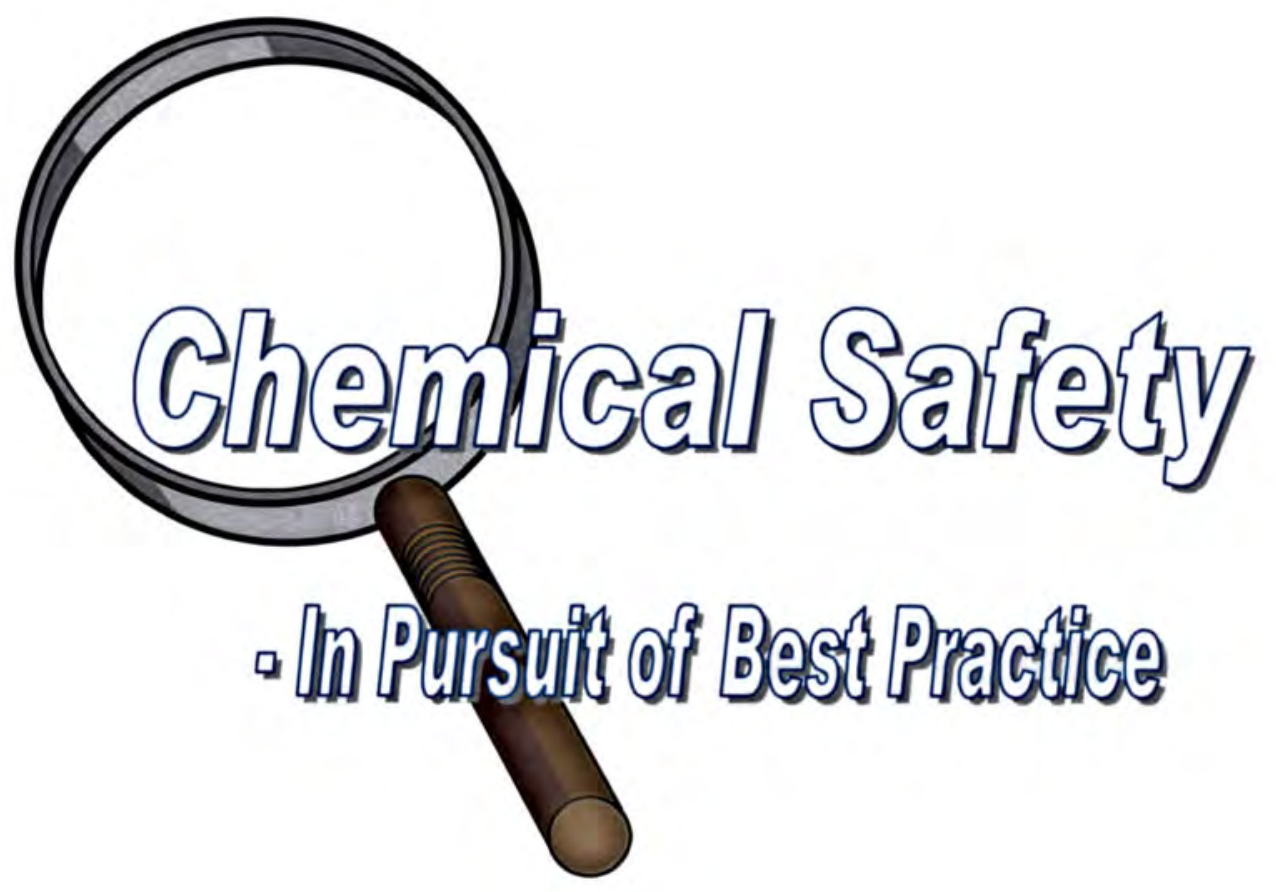

Books 4

- Organilsation of Worth. 
Book 4 is designed to stimulate discussion and gather information on the organisational measures that the site applies to the management of chemical safety. The purpose is to identify innovative, cost effective and practical approaches to chemical safety that go beyond requirements expressed in law.

SITE:

CONTACT PERSON:

\section{A. Leadership}

1. Please rate management visibility and activity in OHS.

\begin{tabular}{|l|l|l|l|}
\hline Poor & Fair & Good & Excellent \\
\hline
\end{tabular}

If "Good" or "Excellent", what are the key initiatives or success factors in your approach?

2. Please rate development, enunciation and deployment of OHS policies, goals and values.

\begin{tabular}{|l|l|l|l|} 
Poor & Fair & Good & Excellent \\
\hline
\end{tabular}

If "Good" or "Excellent", what are the key initiatives or success factors in your approach?

3. Please rate leadership initiatives in monitoring OHS performance.

\begin{tabular}{|l|l|l|l|}
\hline Poor & Fair & Good & Excellent \\
\hline
\end{tabular}

If "Good" or "Excellent", what are the key initiatives or success factors in your approach? 


\section{B. Commitment}

1. Please rate commitment of financial resources to OHS initiatives.

\begin{tabular}{|l|l|l|l|}
\hline Poor & Fair & Good & Excellent \\
\hline
\end{tabular}

If "Good" or "Excellent", what are the key initiatives or success factors in your approach?

2. Please rate deployment of internal resources to support OHS efforts and initiatives.

\begin{tabular}{|l|l|l|l|}
\hline Poor & Fair & Good & Excellent \\
\hline
\end{tabular}

If "Good" or "Excellent", what are the key initiatives or success factors in your approach?

3. Please rate access to external resources for OHS.

\begin{tabular}{|l|l|l|l|}
\hline Poor & Fair & Good & Excellent \\
\hline
\end{tabular}

If "Good" or "Excellent", what are the key initiatives or success factors in your approach?

4. Please rate employee commitment, as derived from involvement and empowerment initiatives.

\begin{tabular}{|c|c|c|c|}
\hline Poor & Fair & Good & Excellent \\
\hline
\end{tabular}

If "Good" or "Excellent", what are the key initiatives or success factors in your approach? 


\section{Planning}

1. Please rate strategic planning for OHS.

\begin{tabular}{|l|l|l|l|}
\hline Poor & Fair & Good & Excellent \\
\hline
\end{tabular}

If "Good" or "Excellent", what are the key initiatives or success factors in your approach?

2. Please rate OHS target-setting efforts and budgetary support for this.

\begin{tabular}{|c|c|c|c|}
\hline Poor & Fair & Good & Excellent \\
\hline
\end{tabular}

If "Good" or "Excellent", what are the key initiatives or success factors in your approach?

3. Please rate alignment of contractor safety plans with Company plans.

\begin{tabular}{|c|c|c|c|}
\hline Poor & Fair & Good & Excellent \\
\hline
\end{tabular}

If "Good" or "Excellent", what are the key initiatives or success factors in your approach?

\section{Organisation}

1. Please rate the organisational structure for OHS delivery on-site (eg. reporting arrangements).

\begin{tabular}{|c|c|c|c|}
\hline Poor & Fair & Good & Excellent \\
\hline
\end{tabular}

If "Good" or "Excellent", what are the key initiatives or success factors in your approach? 
2. Please rate the extent to which OHS is integrated into the overall management system.

\begin{tabular}{|l|l|l|l|}
\hline Poor & Fair & Good & Excellent \\
\hline
\end{tabular}

If "Good" or "Excellent", what are the key initiatives or success factors in your approach?

3. Please rate integration of the efforts of the various departments that play a role in the management of hazardous materials.

\begin{tabular}{|c|c|c|c|}
\hline Poor & Fair & Good & Excellent \\
\hline
\end{tabular}

If "Good" or "Excellent", what are the key initiatives or success factors in your approach?

\section{E. Training}

1. Please rate the arrangements and delivery of induction training programs, as they relate to OHS.

\begin{tabular}{|l|l|l|l|}
\hline Poor & Fair & Good & Excellent \\
\hline
\end{tabular}

If "Good" or "Excellent", what are the key initiatives or success factors in your approach?

2. Please rate the arrangements and delivery of refresher training programs, as they relate to OHS.

\begin{tabular}{|l|l|l|l|}
\hline Poor & Fair & Good & Excellent \\
\hline
\end{tabular}

If "Good" or "Excellent", what are the key initiatives or success factors in your approach? 
3. Please rate the arrangements and delivery of safety representative training programs.

\begin{tabular}{|l|l|l|l|}
\hline Poor & Fair & Good & Excellent \\
\hline
\end{tabular}

If "Good" or "Excellent", what are the key initiatives or success factors in your approach?

4. Plcase rate the arrangements for evaluation of the effectiveness of OHS training delivered on-site.

\begin{tabular}{|l|l|l|l|}
\hline Poor & Fair & Good & Excellent \\
\hline
\end{tabular}

If "Good" or "Excellent", what are the key initiatives or success factors in your approach?

\section{F. Communication}

1. Please rate arrangements and effectiveness of hazard communication efforts onsite.

\begin{tabular}{|c|c|c|}
\hline \multicolumn{2}{|l|}{ Poor } & \\
\hline
\end{tabular}

If "Good" or "Excellent", what are the key initiatives or success factors in your approach?

2. Please rate arrangements and delivery in the area of communication of OHS performance expectations and targets.

\begin{tabular}{|c|c|c|c|}
\hline Poor & Fair & Good & Excellent \\
\hline
\end{tabular}


If "Good" or "Excellent", what are the key initiatives or success factors in your approach?

3. Please rate arrangements and delivery of feedback in relation to performance (against OHS targets).

\begin{tabular}{|l|l|l|l|}
\hline Poor & Fair & Good & Excellent \\
\hline
\end{tabular}

If "Good" or "Excellent", what are the key initiatives or success factors in your approach?

\section{G. Measurement}

1. Please rate the use of "lagging" indicators of OHS performance on-site.

\begin{tabular}{|l|l|l|l|}
\hline Poor & Fair & Good & Excellent \\
\hline
\end{tabular}

If "Good" or "Excellent", what are the key initiatives or success factors in your approach?

2. Please rate the use of "leading" indicators of OHS performance on-site.

\begin{tabular}{|l|l|l|l|}
\hline Poor & Fair & Good & Excellent \\
\hline
\end{tabular}

If "Good" or "Excellent", what are the key initiatives or success factors in your approach? 
* This research forms part of PhD studies being undertaken by Barry Chesson, Manager - Occupational Hygiene, Alcoa World Alumina Australia. The studies are being pursued though the Faculty of Communications, Health and Science at Edith Cowan University (Joondalup Campus).

- The full study title is "In Pursuit of Best Practice: Benchmarking Tools and Processes for the Management of Hazardous Substances in the Workplace."

* This study is seeking to develop and test new tools and processes to make benchmarking activity and the transfer of technology, ideas and approaches more efficient and meaningful. It will focus on the management of chemicals within the Western Australian Mining Industry, but outcomes should be applicable within the wider framework of OHS and to other industry sectors.

* It will draw heavily from state-of-the-art management theory and will seek to establish the linkage between the people factor, the workplace environment factor and the organisation of work factor as they contribute to workplace health and safety performance.

* Six booklets will be used to assist with the gathering of data for this project. A further two booklets will be used for summarising results and providing feedback to participating sites. The booklets are as follows:
Book 1 Site Profile
Book 2 Preliminary Questions
Book 3 Compliance
Book 4 Organisation of Work
Book 5 The Workplace Environment
Book 6 People Initiatives
Book 7 Results - Site strengths and opportunities for improvement
Book 8 Scoresheets, inter-site comparisons and benchmarking opportunities

- Study outcomes will assist with the selection of benchmarking partners and will help to identify "pockets of excellence" for focused attention. This will encourage and assist organisations to take steps towards identifying and implementing Industry best practice in the OHS element of interest.

* For further information please contact the researcher, Barry Chesson, on (08) 94103301 (W) or $\quad$ or on emmail at barry.chesson@alcoa.com.au.

(c) 


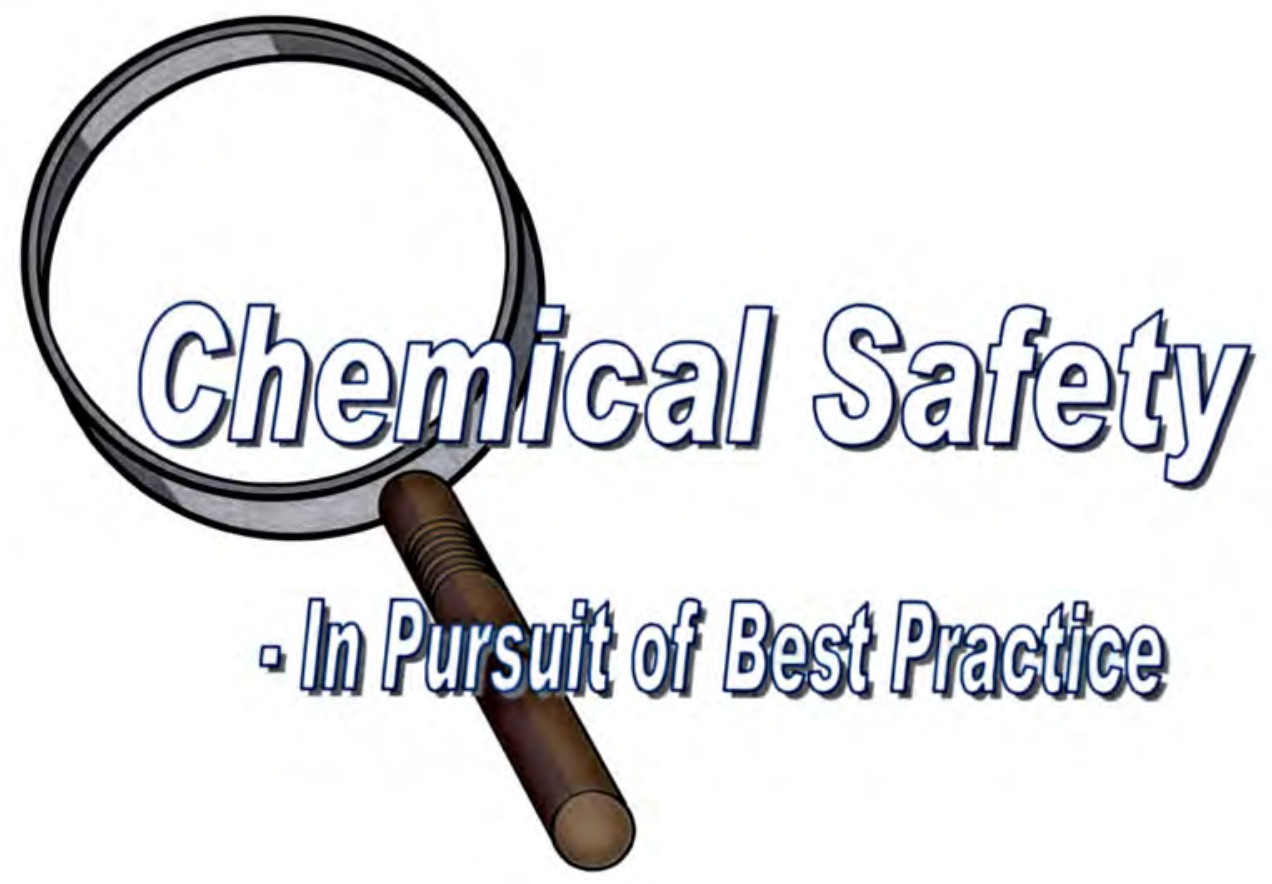

Books

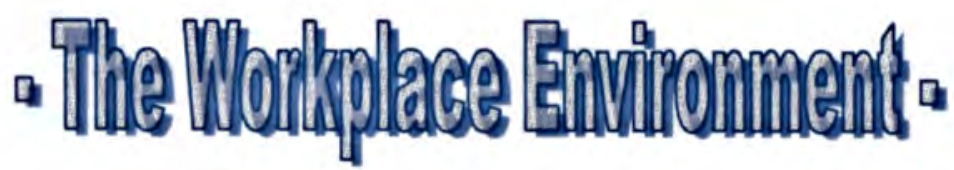

Barry Chesson

September 2002 
Book 5 is designed to stimulate discussion and gather information on how the site addresses chemical safety issues that relate to the workplace environment factor. The purpose is to identify innovative, cost effective and practical approaches that go beyond requirements expressed in law.

SITE: CONTACT PERSON:

\section{A. HAZARD IDENTIFICATION}

1. If a walk-through survey program exists, please rate the effectiveness of this.

\begin{tabular}{|c|c|c|c|}
\hline Poor & Fair & Good & Excellent \\
\hline
\end{tabular}

If "Good" or "Excellent", what are the key initiatives or success factors in your approach?

2. If job dictionaries exist, please rate the effectiveness of these.

\begin{tabular}{|c|c|c|c|}
\hline Poor & Fair & Good & Excellent \\
\hline
\end{tabular}

If "Good" or "Excellent", what are the key initiatives or success factors in your approach?

3. If chemical inventories have been developed for the site, please rate the effectiveness of these.

\begin{tabular}{|c|c|c|c|}
\hline Poor & Fair & Good & Excellent \\
\hline
\end{tabular}

If "Good" or "Excellent", what are the key initiatives or success factors in your approach? 


\section{B. RISK ASSESSMENT}

1. If sampling plans and schedules exist, please rate the effectiveness of these.

\begin{tabular}{|l|l|l|l|}
\hline Poor & Fair & Good & Excellent \\
\hline
\end{tabular}

If "Good" or "Excellent", what are the key initiatives or success factors in your approach?

2. If a qualitative exposure assessment program exists, please rate the effectiveness of this.

\begin{tabular}{|l|l|l|l|}
\hline Poor & Fair & Good & Excellent \\
\hline
\end{tabular}

If "Good" or "Excellent", what are the key initiatives or success factors in your approach?

3. If a quantitative exposure assessment program exists, please rate the effectiveness of this.

\begin{tabular}{|c|c|c|c|}
\hline Poor & Fair & Good & Excellent \\
\hline
\end{tabular}

If "Good" or "Excellent", what are the key initiatives or success factors in your approach?

4. If sampling results are subject to statistical treatment or analysis, please rate the effectiveness of this.

\begin{tabular}{|c|c|c|c|}
\hline Poor & Fair & Good & Excellent \\
\hline
\end{tabular}

If "Good" or "Excellent", what are the key initiatives or success factors in your approach? 
5. If an exposure baseline has been established, please rate the effectiveness of this.

\begin{tabular}{|c|c|c|c|}
\hline Poor & Fair & Good & Excellent \\
\hline
\end{tabular}

If "Good" or "Excellent", what are the key initiatives or success factors in your approach?

6. If a record-keeping system is in place, please rate the effectiveness of this.

\begin{tabular}{|c|c|c|c|}
\hline Poor & Fair & Good & Excellent \\
\hline
\end{tabular}

If "Good" or "Excellent", what are the key initiatives or success factors in your approach?

\section{RISK CONTROL}

1. Please rate processes for feasibility assessment and priority setting (for OHS controls).

\begin{tabular}{|c|c|c|c|}
\hline Poor & Fair & Good & Excellent \\
\hline
\end{tabular}

If "Good" or "Excellent", what are the key initiatives or success factors in your approach?

2. Please rate the effectiveness of substitution/elimination activity.

\begin{tabular}{|c|c|c|c|}
\hline Poor & Fair & Good & Excellent \\
\hline
\end{tabular}

If "Good" or "Excellent", what are the key initiatives or success factors in your approach? 
3. Please rate the effectiveness of procurement controls.

\begin{tabular}{|c|c|c|c|}
\hline Poor & Fair & Good & Excellent \\
\hline
\end{tabular}

If "Good" or "Excellent", what are the key initiatives or success factors in your approach?

4. Please rate the effectiveness of engineering controls.

\begin{tabular}{|c|c|c|c|}
\hline Poor & Fair & Good & Excellent \\
\hline
\end{tabular}

If "Good" or "Excellent", what are the key initiatives or success factors in your approach?

5. Please rate the effectiveness of administrative/work practice controls.

\begin{tabular}{|l|l|l|l|}
\hline Poor & Fair & Good & Excellent \\
\hline
\end{tabular}

If "Good" or "Excellent", what are the key initiatives or success factors in your approach?

6. Please rate the effectiveness of PPE controls.

\begin{tabular}{|c|c|c|c|}
\hline Poor & Fair & Good & Excellent \\
\hline
\end{tabular}

If "Good" or "Excellent", what are the key initiatives or success factors in your approach? 


\begin{tabular}{|c|c|}
\hline $\begin{array}{l}\text { IH Program } \\
\text { Development } \\
\text { Stage III } \\
\text { (advanced) }\end{array}$ & 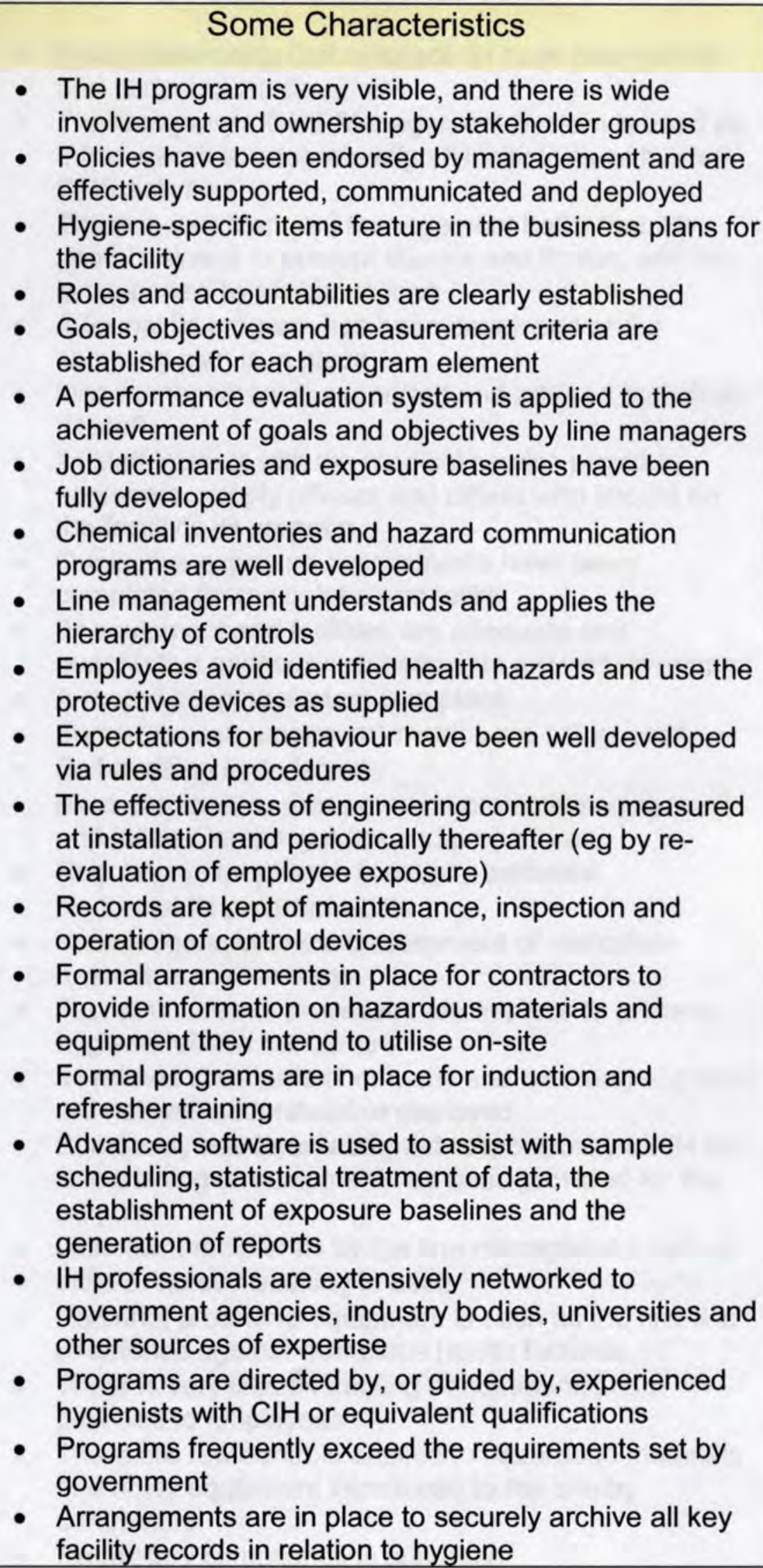 \\
\hline
\end{tabular}




\begin{tabular}{|c|c|}
\hline $\begin{array}{l}\text { IH Program } \\
\text { Development } \\
\text { Stage II } \\
\text { (improving) }\end{array}$ & 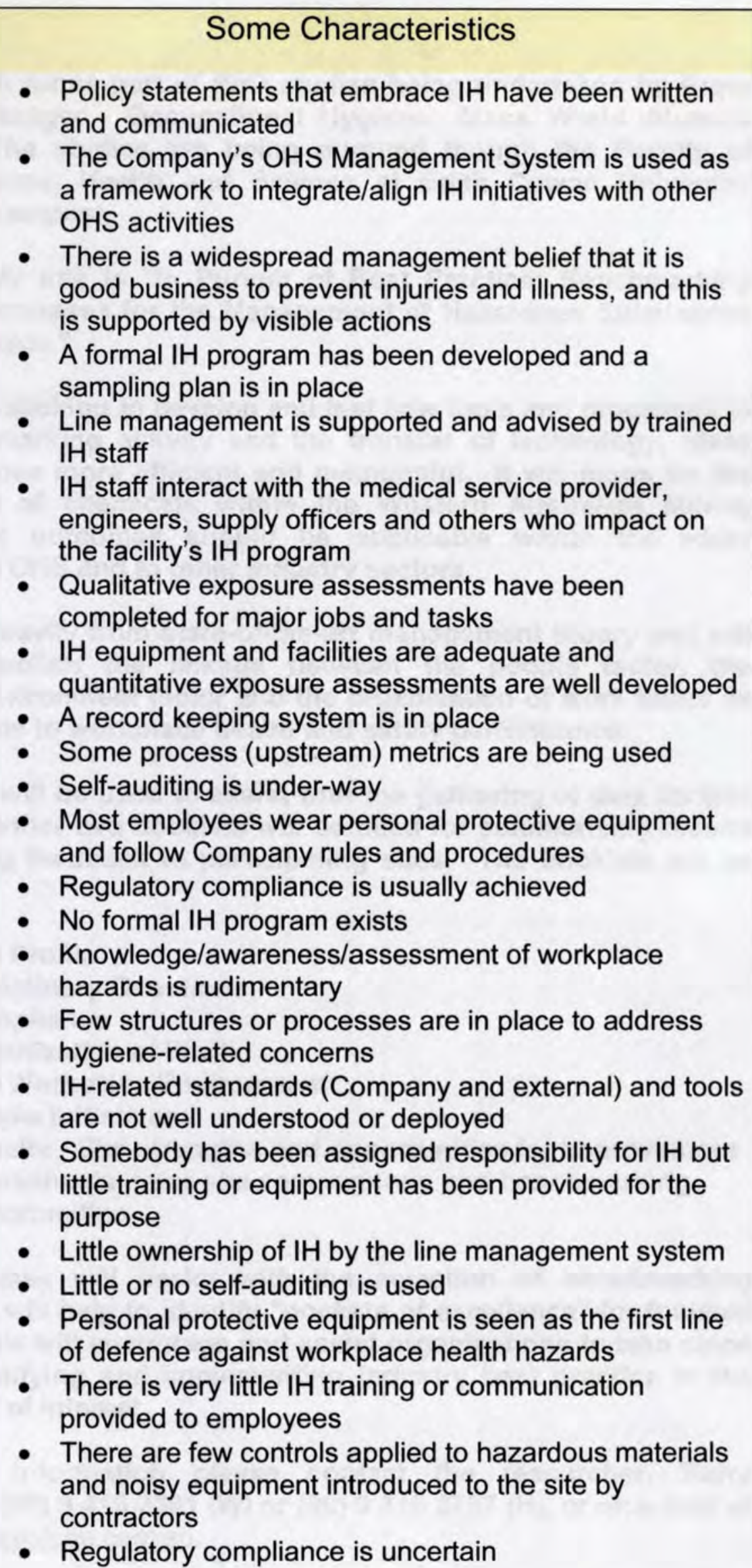 \\
\hline
\end{tabular}


This research forms part of PhD studies being undertaken by Barry Chesson, Manager - Occupational Hygiene, Alcoa World Alumina Australia. The studies are being pursued though the Faculty of Communications, Health and Science at Edith Cowan University (Joondalup Campus).

* The full study title is "In Pursuit of Best Practice: Benchmarking Tools and Processes for the Management of Hazardous Substances in the Workplace."

* This study is seeking to develop and test new tools and processes to make benchmarking activity and the transfer of technology, ideas and approaches more efficient and meaningful. It will focus on the management of chemicals within the Western Australian Mining Industry, but outcomes should be applicable within the wider framework of OHS and to other industry sectors.

* It will draw heavily from state-of-the-art management theory and will seek to establish the linkage between the people factor, the workplace environment factor and the organisation of work factor as they contribute to workplace health and safety performance.

- Six booklets will be used to assist with the gathering of data for this project. A further two booklets will be used for summarising results and providing feedback to participating sites. The booklets are as follows:

Book 1 Site Profile

Book 2 Preliminary Questions

Book 3 Compliance

Book 4 Organisation of Work

Book 5 The Workplace Environment

Book 6 People Initiatives

Book 7 Results - Site strengths and opportunities for improvement

Book 8 Scoresheets, inter-site comparisons and benchmarking opportunities

* Study outcomes will assist with the selection of benchmarking partners and will help to identify "pockets of excellence" for focused attention. This will encourage and assist organisations to take steps towards identifying and implementing Industry best practice in the OHS element of interest.

* For further information please contact the researcher, Barry Chesson, on (08) 94103301 (W) or $\quad$ or on e-mail at barry.chesson@alcoa.com.au.

(C) 


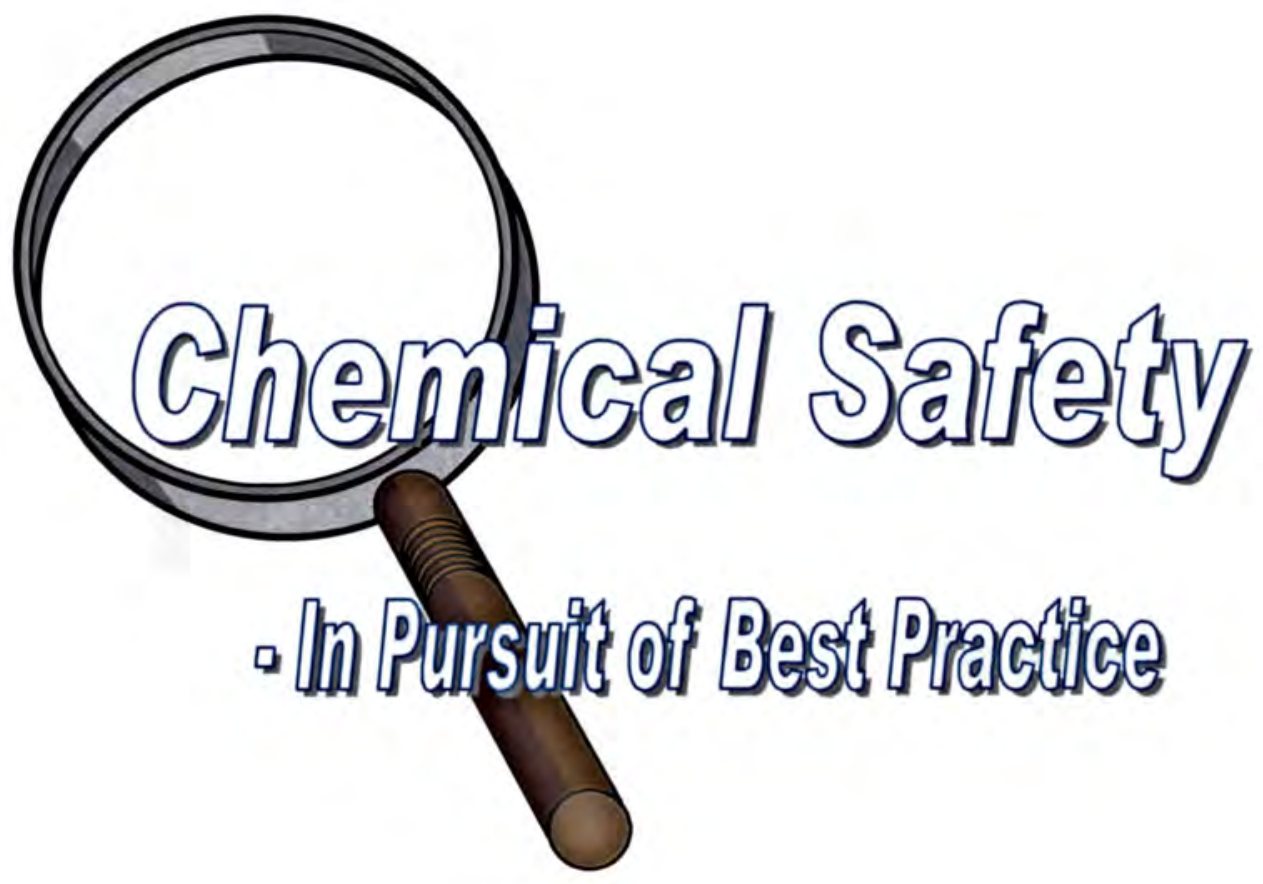

\section{Book 6}

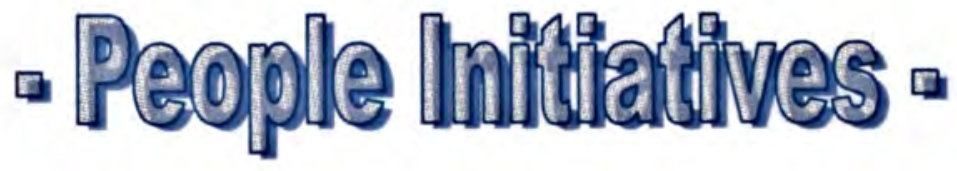


Book 6 is designed to stimulate discussion and gather information on how the site addresses issues that relate to the people factor. The purpose is to identify innovative, cost effective and practical approaches that impact on chemical safety and go beyond the requirements expressed in law.

\section{SITE:}

\section{CONTACT PERSON:}

1. If a behavioural safety program exists, please rate this.

\begin{tabular}{|l|l|l|l|}
\hline Poor & Fair & Good & Excellent \\
\hline
\end{tabular}

If "Good" or "Excellent", what are the key initiatives or success factors in your approach?

2. If a task observation program exists (within a behavioural safety program), please rate this.

\begin{tabular}{|c|c|c|c|}
\hline Poor & Fair & Good & Excellent \\
\hline
\end{tabular}

If "Good" or "Excellent", what are the key initiatives or success factors in your approach?

3. If motivation and recognition efforts exist in a formal way, please rate these in terms of usefulness.

\begin{tabular}{|c|c|c|c|}
\hline Poor & Fair & Good & Excellent \\
\hline
\end{tabular}

If "Good" or "Excellent", what are the key initiatives or success factors in your approach?

4. If attitude surveys have been conducted, please rate these in terms of usefulness.

\begin{tabular}{|c|c|c|c|}
\hline Poor & Fair & Good & Excellent \\
\hline
\end{tabular}


If "Good" or "Excellent", what are the key initiatives or success factors in your approach?

5. If morale surveys have been conducted, please rate these in terms of usefulness.

\begin{tabular}{|l|l|l|l|}
\hline Poor & Fair & Good & Excellent \\
\hline
\end{tabular}

If "Good" or "Excellent", what are the key initiatives or success factors in your approach?

6. If a safety (management) contact program exists, please rate this in terms of usefulness.

\begin{tabular}{|l|l|l|l|}
\hline Poor & Fair & Good & Excellent \\
\hline
\end{tabular}

If "Good" or "Excellent", what are the key initiatives or success factors in your approach?

7. If PPE compliance surveys are undertaken, please rate these in terms of usefulness.

\begin{tabular}{|l|l|l|l|}
\hline Poor & Fair & Good & Excellent \\
\hline
\end{tabular}

If "Good" or "Excellent", what are the key initiatives or success factors in your approach?

8. Please rate sitc promotional efforts for OHS.

\begin{tabular}{|c|c|c|c|}
\hline Poor & Fair & Good & Excellent \\
\hline
\end{tabular}

If "Good" or "Excellent", what are the key initiatives or success factors in your approach? 
* This research forms part of PhD studies being undertaken by Barry Chesson, Manager - Occupational Hygiene, Alcoa World Alumina A.ustralia. The studies are being pursued though the Faculty of Communications, Health and Science at Edith Cowan University (Joondalup Campus).

* The full study vitle is "In Pursuit of Best Practice: Benchmarking Tools and Processes for the Management of Hazardous Substances in the Workplace."

* This study is seeking to develop and test new tools and processes to make benchmarking activity and the transfer of technology, ideas and approachos more efficient and meaningful. It will focus on the management of chemicals within the Western Australian Mining Industry, but outcomes should be applicable within the wider framework of OHS and to other industry sectors.

* It will draw heavily from state-of-the-art management theory and will seek to establish the linkage between the people factor, the workplace environment factor and the organisation of work factor as they contribute to workplace health and safety performance.

* Six booklets will be used to assist with the gathering of data for this project. A further two booklets will be used for summarising results and providing feedback to participating sites. The booklets are as follows:
Book 1 Site Profile
Book 2 Preliminary Questions
Book 3 Compliance
Book 4 Organisation of Work
Book 5 The Workplace Environment
Book 6 People Initiatives
Book 7 Results - Site strengths and opportunities for improvement
Book 8 Scoresheets, inter-site comparisons and benchmarking opportunities

* Study outcomes will assist with the selection of benchmarking partners and will help to identify "pockets of excellence" for focused attention. This will encourage and assist organisations to take steps towards identifying and implementing Industry best practice in the OHS element of interest.

* For further information please contact the researcher, Barry Chesson, on (08) 94103301 (W) or ), or on e-mail at barry.chesson@alcoa.com.au.

(c) 

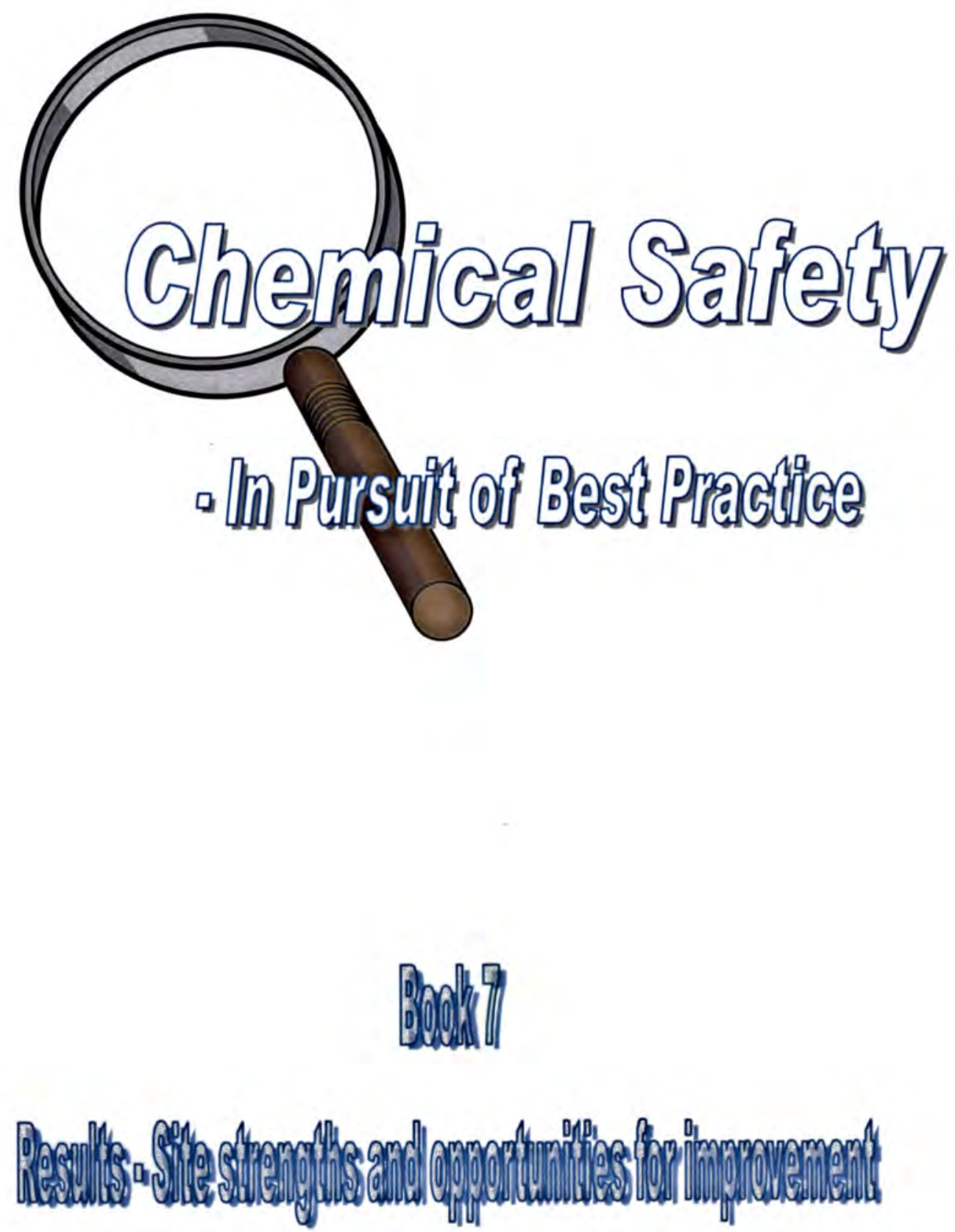
Book 7 is designed to provide the location with an analysis of data gathered during the preliminary contact phase and during subsequent site visit(s). It presents an appraisal of programs and processes relating to $\mathrm{OHS}$ in general, and chemical safety, in particular. Feedback is provided in terms of both strengths and opportunities for improvement.

SITE:

CONTACT PERSON:

1. OHS - AN OVERVIEW

2. CHEMICAL SAFETY - AN OVERVIEW 
3. COMPLIANCE

$3.1 \quad$ Strengths

3.2 Opportunities for Improvement 
4. ORGANISATION OF WORK

(Beyond Compliance - Management Enablers)

4.1 Strengths

4.2 Opportunities for Improvement 
5. THE WORKPLACE ENVIRONMENT

(Beyond Compliance - Technical Initiatives)

\subsection{Strengths}

5.2 Opportunities for Improvement 
6. PEOPLE

(Beyond Compliance - People Initiatives)

6.1 Strengths

6.2 Opportunities for Improvement 
7. SUMMARY AND RECOMMENDATIONS

\section{Facility Profile}

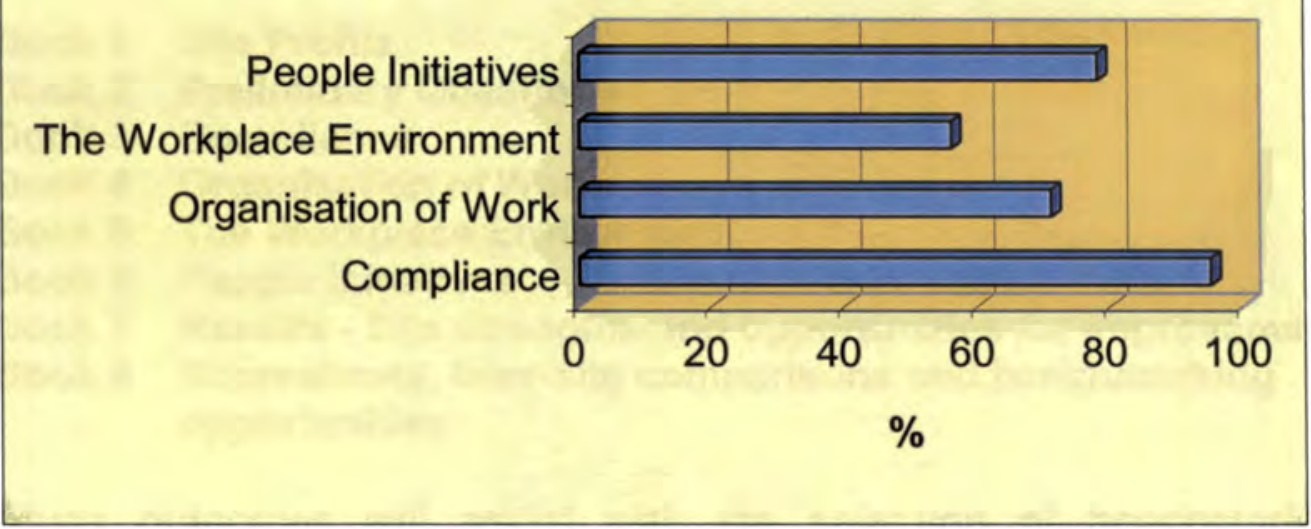


* This research forms part of PhD studies being undertaken by Barry Chesson, Manager - Occupational Hygiene, Alcoa World Alumina Australia. The studies are being pursued though the Faculty of Communications, Health and Science at Edith Cowan University (Joondalup Campus).

* The full study title is "In Pursuit of Best Practice: Benchmarking Tools and Processes for the Management of Hazardous Substances in the Workplace."

* This study is seeking to develop and test new tools and processes to make benchmarking activity and the transfer of technology, ideas and approaches more efficient and meaningful. It will focus on the management of chemicals within the Western Australian Mining Industry, but outcomes should be applicable within the wider framework of OHS and to other industry sectors.

* It will draw heavily from state-of-the-art safety management theory and will seek to establish the linkage between the organisation of work factor, the workplace environment factor and the people factor as they contribute to workplace health and safety performance.

* Six booklets will be used to assist with the gathering of data for this project. A further two booklets will be used for summarising results and providing feedback to participating sites. The booklets are as follows:

\section{Book 1 Site Profile}

Book 2 Preliminary Questions

Book 3 Compliance

Book 4 Organisation of Work

Book 5 The Workplace Environment

Book 6 People Initiatives

Book 7 Results - Site strengths and opportunities for improvement

Book 8 Scoresheets, inter-site comparisons and benchmarking opportunities

* Study outcomes will assist with the selection of benchmarking partners and will help to identify "pockets of excellence" for focused attention. This will encourage and assist organisations to take steps towards identifying and implementing Industry best practice in the OHS element of interest.

* For further information please contact the researcher, Barry Chesson, on (08) 94103301 (W) or $\quad$ or on e-mail at (ㄷ) barry.chesson@alcoa.com.au. 

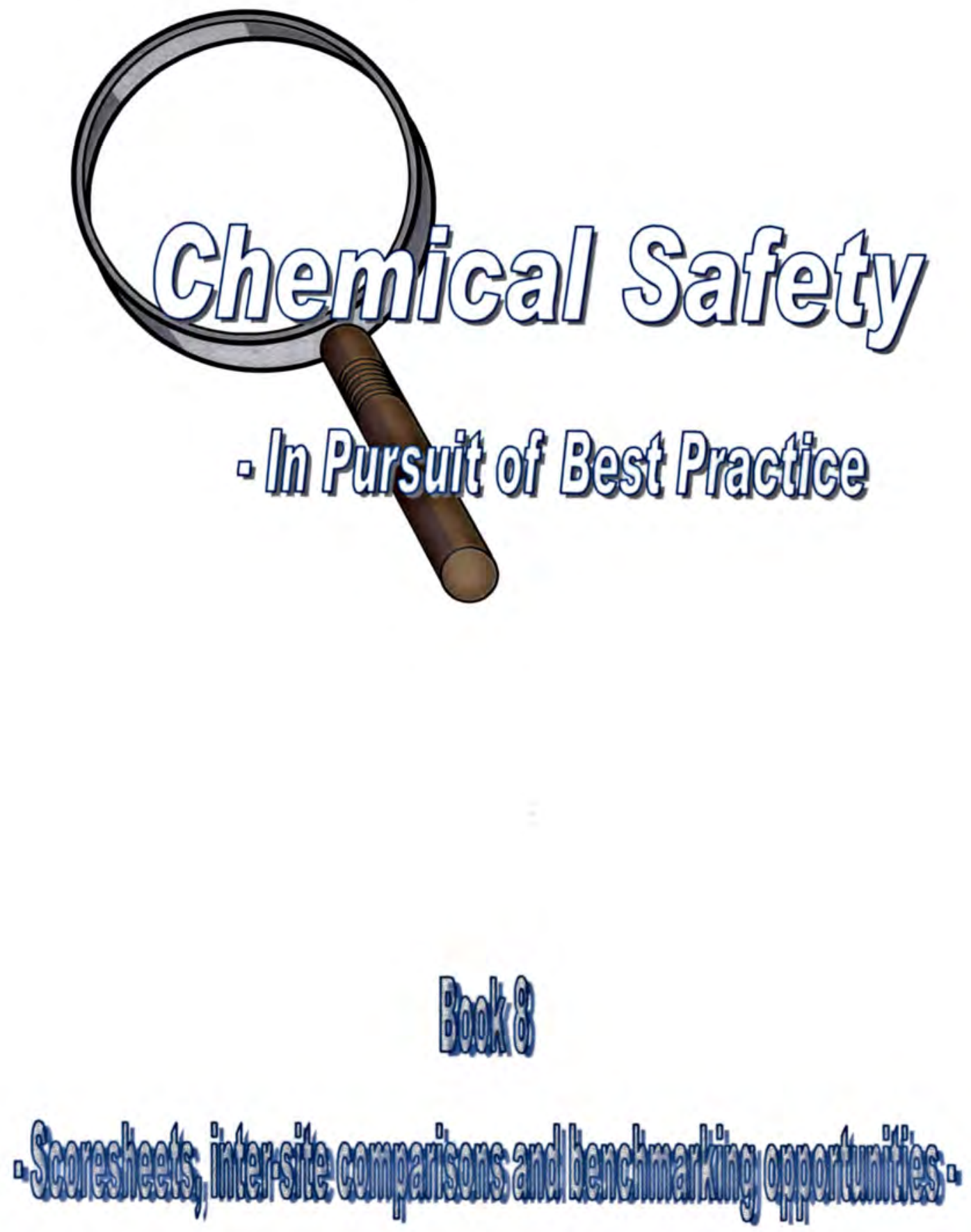

Barry Chesson September 2002 
Book 8 is the final booklet in the series. It is designed to provide scoresheets and graphical representations of the status of OHS and chemical safety programs and processes at the facility. In addition, intersite comparisons are provided and benchmarking opportunities are identified. Book 8 will be made available to participating sites after a full analysis of data has been completed.

\section{SITE:}

CONTACT PERSON:

\section{A. COMPARISONS WITH OTHER PARTICIPATING SITES}

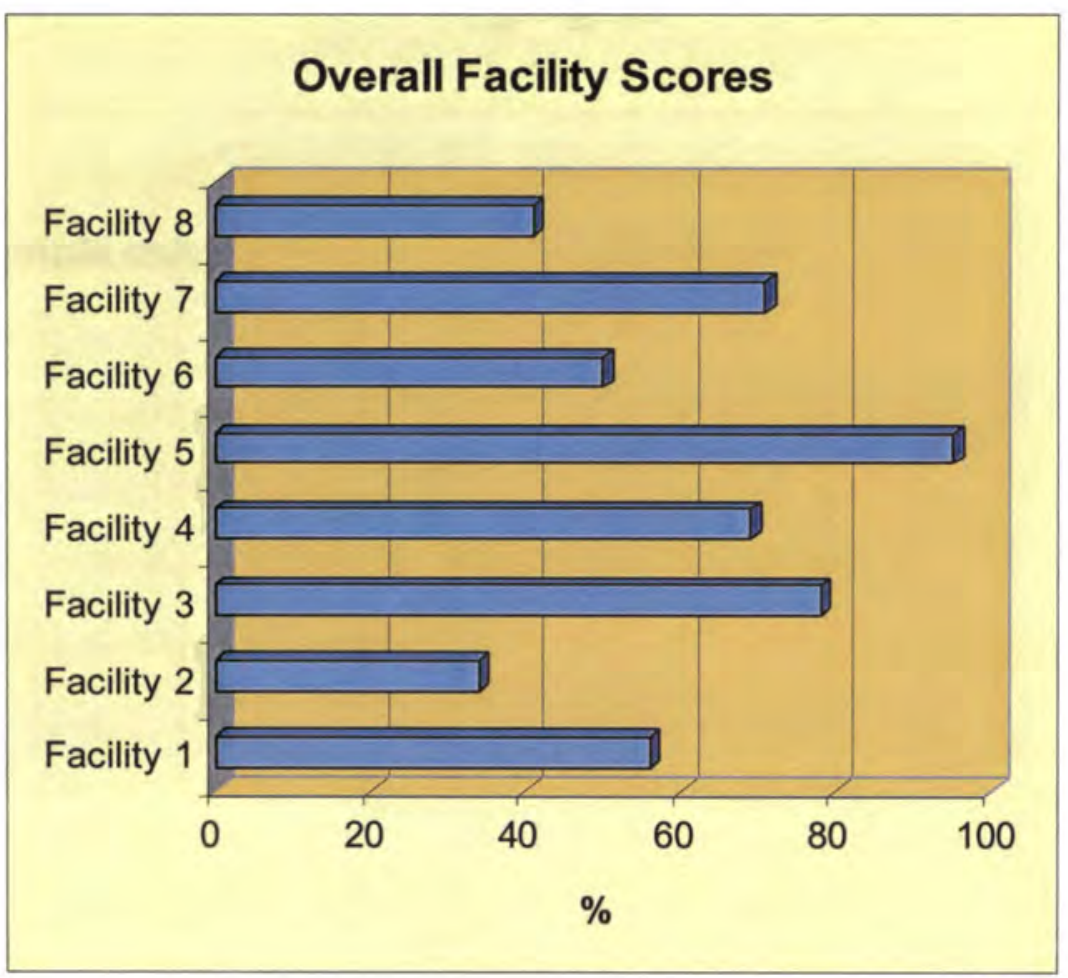

(sample only) 


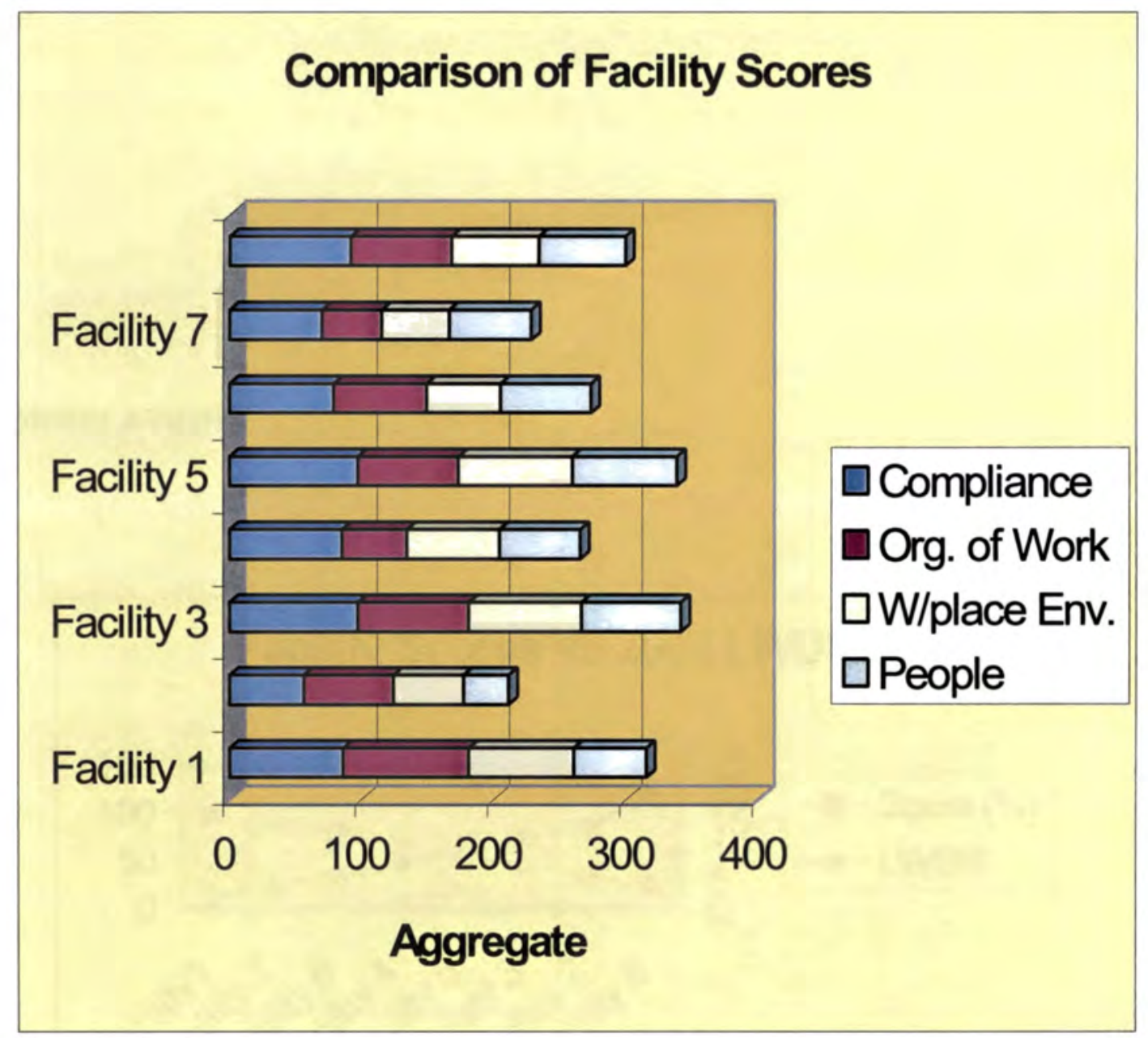

(sample only) 
B. CORRELATIONS

\section{Facility Scores vs 2002 LWDRs}

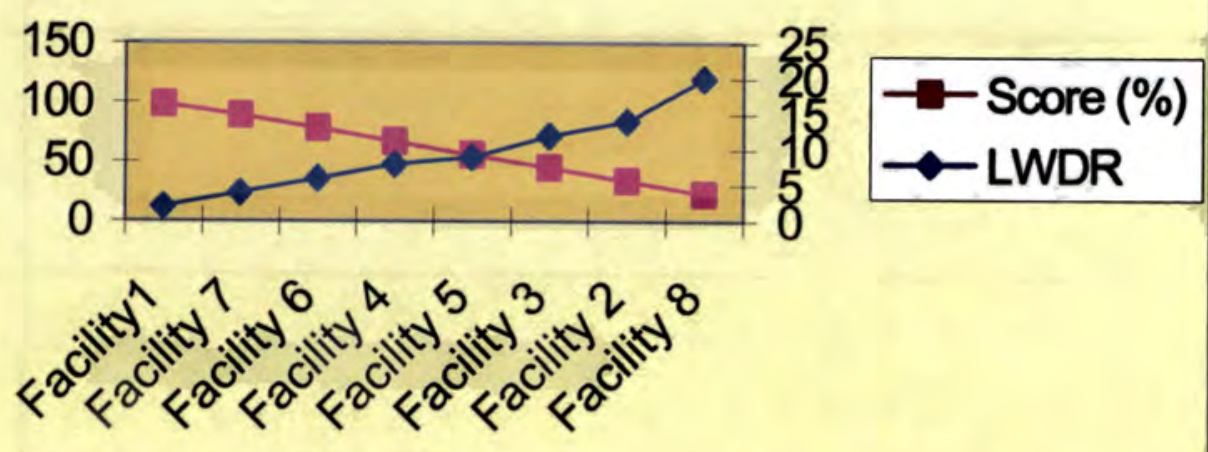

(sample only) 
C. BENCHMARKING OPPORTUNITIES - OHS GENERAL

\begin{tabular}{|c|c|}
\hline Facility & Benchmarking Opportunities \\
\hline 1 & \\
\hline 2 & \\
\hline 3 & \\
\hline 4 & \\
\hline 5 & \\
\hline 6 & \\
\hline
\end{tabular}


D. BENCHMARKING OPPORTUNITIES - CHEMICAL SAFETY

\begin{tabular}{|c|c|}
\hline Facility & Benchmarking Opportunities \\
\hline 1 & \\
\hline 2 & \\
\hline 3 & \\
\hline 4 & \\
\hline 5 & \\
\hline 6 & \\
\hline
\end{tabular}


E. SUMMARY \& RECOMMENDATIONS 
* This research forms part of PhD studies being undertaken by Barry Chesson, Manager - Occupational Hygiene, Alcoa World Alumina Australia. The studies are being pursued though the Faculty of Communications, Health and Science at Edith Cowan University (Joondalup Campus).

* The full study title is "In Pursuit of Best Practice: Benchmarking Tools and Processes for the Management of Hazardous Substances in the Workplace."

* This study is seeking to develop and test new tools and processes to make benchmarking activity and the transfer of technology, ideas and approaches more efficient and meaningful. It will focus on the management of chemicals within the Western Australian Mining Industry, but outcomes should be applicable within the wider framewcrk of $\mathrm{OHS}$ and to other industry sectors.

* It will draw heavily from state-of-the-art safety management theory and will seek to establish the linkage between the organisation of work factor, the workplace environment factor and the people factor as they contribute to workplace health and safety performance.

* Six booklets will be used to assist with the gathering of data for this project. A further two booklets will be used for summarising results and providing feedback to participating sites. The booklets are as follows:

\section{Book 1 Site Profile}

Book 2 Preliminary Questions

Book 3 Compliance

Book 4 Organisation of Work

Book 5 The Workplace Environment

Book 6 People Initiatives

Book 7 Results - Site strengths and opportunities for improvement

Book 8 Scoresheets, inter-site comparisons and benchmarking opportunities

Study outcomes will assist with the selection of benchmarking partners and will help to identify "pockets of excellence" for focused attention. This will encourage and assist organisations to take steps towards identifying and implementing Industry best practice in the OHS element of interest.

* For further information please contact the researcher, Barry Chesson, on (08) $94103301(W)$ or $\quad$ or on e-mail at barry.chesson@alcoa.com.au.

(c) 
APPENDIX 8

SAMPLE REPORT TO SITE MANAGEMENT 

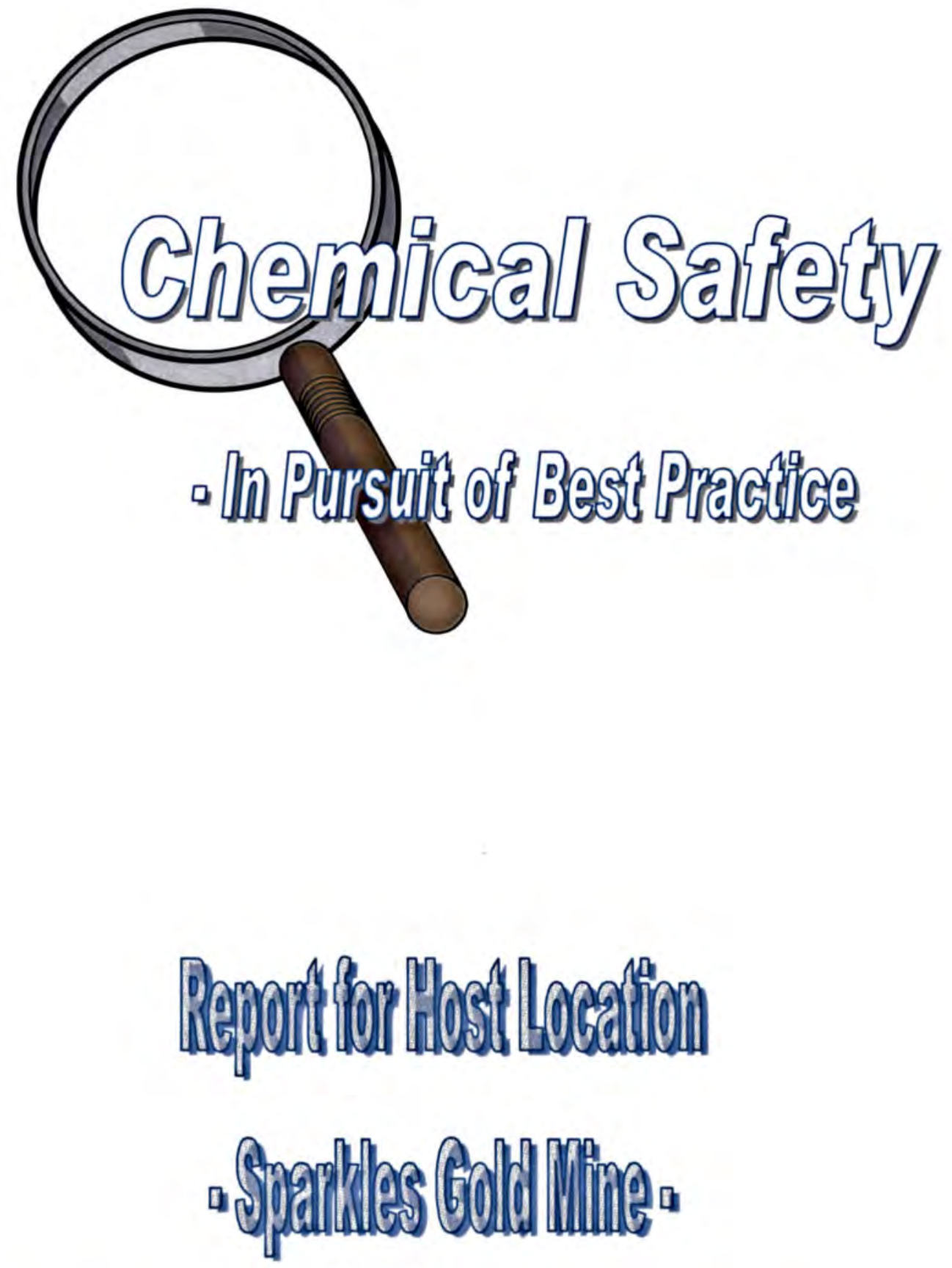

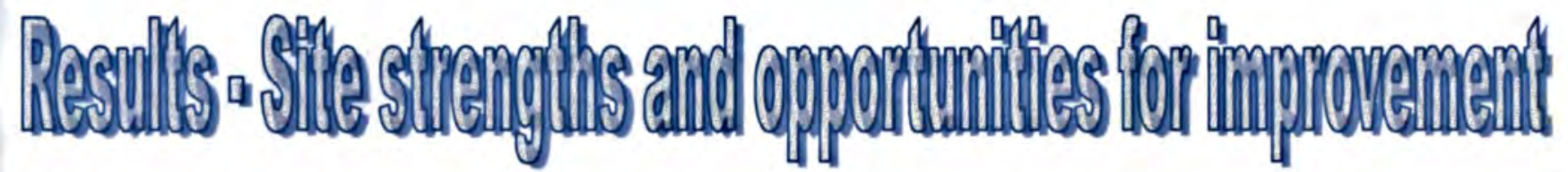

\author{
Barry Chesson
}

February 2003 
This Booklet is designed to provide the location with an analysis of data gathered during the preliminary contact phase of the benchmarking project and during subsequent site visit(s). It presents an appraisal of site programs and processes relating to OHS in general, and chemical safety, in particular. Feedback is provided in terms of both strengths and opportunities for improvement.

SITE: Sparkles Gold Mine CONTACT PERSON: John Smith EHS Supervisor

\section{OHS - AN OVERVIEW}

- Sparkles Gold Mine is an open-cut mine that has been operating for eight years. There are 85 direct employees and 105 contractor personnel onsite. Employees work 12-hour shifts on a fly-in, fly-out basis, with four weeks on-site duty in every six-week period. The facility has an EHS Supervisor, who reports to the Mine Manager. There are 12 elected Safety Representatives on the mine who act in support of the EHS Supervisor.

- Principal occupational health challenges and issues in the physical hazard area are noise and vibration, and to a lesser extent heat stress and fatigue. Chemical hazards are related to ore dust, cyanide materials, mineral acids, cleaning agents, fuels, oils and lubricants, gases, adhesives, solvents and other workshop materials. Ergonomic hazards are, in the main, associated with operation and maintenance of heavy mobile equipment and portable tools, together with activities that involve lifting, pulling, pushing, twisting, awkward postures, repetitive motion and similar.

- The site has not been involved in any formalised benchmarking activity in the past, although there have been meetings within the wider organisation to discuss initiatives and to develop corporate plans for OHS.

- OHS performance measurement is focused on the traditional Lost Time Injury Frequency Rate. The site also tracks severity and restricted injury rates.

\section{CHEMICAL SAFETY - AN OVERVIEW}

- The EHS Supervisor is the principal source of advice at the site on chemical safety matters. The main item of attention is sodium cyanide, as a raw material and as a reagent in the facility's process stream. Management of cyanide risk includes engineering, administrative and PPE 
controls, together with signage, area alarms, training and other aspects. The facility has a system in place to manage the array of raw materials, process chemicals, end products, waste materials and other smaller volume substances needed for the day-to-day operations. Contractors are expected to meet similar requirements/standards with respect to any hazardous materials they bring onto the site.

\section{COMPLIANCE}

\subsection{Strengths}

- A chemical inventory is maintained for the site and is up-to-date.

- Tanks, pipe-work and process vessels containing acid are separated from the cyanide solutions used for gold treatment.

- A Material Safety Data Sheet register is maintained and is freely available to the workforce. This contains MSDSs for all site chemicals, together with copies of risk assessments that have been carried out on the more important of the hazardous substances used on site.

- The hierarchy of controls appears to be widely understood on-site and is well deployed.

- Packaging is labelled in accordance with the ADG Code.

- There are suitable arrangements in place with respect to the selection, storage, maintenance and use of personal protective equipment.

- Statutory obligations with respect to atmospheric monitoring are being met.

- Health surveillance activities meet the requirements of the Regulations.

- First aid procedures and facilities are fully in place to deal with emergencies involving cyanide, corrosive substances or other toxic materials. Each shift has on it 10 people trained to St John Ambulance Certificate II level.

\subsection{Opportunities for Improvement}

- Some MSDSs are out of date (more than 5 years since date of issue) and some are not in the Worksafe Australia format, as required.

- Some weaknesses were seen with respect to the currency and adequacy of MSDSs for materials introduced to the site by contractors. 
- Some deficiencies were observed in relation to labels applied after transfer of hazardous substances to smaller on-site containers.

- There is potential for a higher percentage of containers to be returned to the supplier.

\section{ORGANISATION OF WORK (Beyond Compliance - Management Enablers)}

\subsection{Strengths}

- The Mine Manager is highly visible in his support for OHS. Policy statements, goals and values are well developed, expressed and deployed at the site.

- There is a sound record at the site in relation to commitment of financial and human resources to worthwhile OHS projects.

- Engagement of the workforce in OHS initiatives is achieved in the main via the site's OHS Committee structure, the elected Safety Representatives on each shift, and via toolbox meetings and other forms of interaction at work.

- Site leadership is involved, with others, in the broader Company's strategic planning for $\mathrm{OHS}$, in setting targets and in monitoring performance in relation to these.

- Contractor safety plans are reviewed reguiarly with the Mine Manager and the OHS Supervisor.

- The MARCSTA one-day package is the Mining Industry's standard for induction training. All employees, including contractors, are required to have a MARCSTA Certificate before they can work on the Sparkles site. A half-day, local area induction program supplements what has been covered in the MARCSTA program.

" The OHS Supervisor devises and implements other forms of OHS training at the site. Training plans are developed 12 months in advance of deployment. Packages are made available to Supervisors and Safety Representatives for use in crew safety meetings.

\subsection{Opportunities for Improvement}


- There is a relatively large commitment at the site to OHS training. However, the facility might care to consider introducing some means of evaluating the effectiveness of the OHS training that is delivered on-site.

- Colisider the introduction of some leading indicators of performance. These should be measures that are easy to collect, understand and review, that are meaningful and relate readily to key management enablers for OHS, and are easily represented in reports or other forms of communication.

\section{THE WORKPLACE ENVIRONMENT (Beyond Compliance - Technical Initiatives)}

\section{$5.1 \quad$ Strengths}

- Workplace atmospheric monitoring meets and exceeds that established by CONTAM requirements of the Department of Minerals and Petroleum Resources. Sampling is carried out by a contract Industrial Hygiene organisation - using methods that meet Worksafe Australia criteria. Results are shared with the Site OHS Committee.

- HAZOP tools were applied at the original design stage for the mine and during a major upgrade two years ago.

- Maintenance and production crews have developed and implemented several smaller scale engineering controls/innovations. Some of these may be of interest to a benchmarking partner.

- The site has well documented job/task descriptions and procedures, with safety measures built into these. Job Safety Analyses (JSAs) are used routinely at the facility.

- There are significant levels of training and awareness-building effort applied to the deployment of key hazardous substances at the site.

\subsection{Opportunities for Improvement}

- Consider establishing an exposure baseline - so that typical exposures (to airborne contaminants) can be assigned to all work groups at the mine. Training, awareness-building and communication opportunities will arise from this.

- There is potential for the site (and the wider organisation) to utilise a version of the bar code technology that has been applied elsewhere to keep chemical inventories up-to-date, to assist with the implementation of 
MSDS programs, to control unauthorised use of chemicals and to better track deployment and use of these materials.

6. PEOPLE

(Beyond Compliance - People Initiatives)

\subsection{Strengths}

- The Site has sought to introduce a behavioural safety program. This is in the early stages of deployment and is being supported by consultants with experience in introducing such programs into Western Australian mine sites. The program has good support and engagement from Site Management and supervisors.

- A Site committee is responsible for safety promotion activities at the location. There is an imaginative program of events for the next 12 months and Site Management has provided the material resources to allow the committee to function effectively.

- An attitude/perception survey was carried out in 2000 . This was to establish what the workforce thought of local safety efforts and to attempt to identify barriers to good safety periormance at the location. Results were communicated widely and also used in planning subsequent OHS activities.

- Several PPE compliance surveys have been carried out over the past three years. Results have formed the basis of a number of toolbox safety sessions and some improvements have been noted in the aftermath of these.

\subsection{Opportunities for Improvement}

- The early efforts to introduce a behavioural safety program on site are commendable. However, a top-down approach has been used and unless there is more engagement of the workforce in the program there will be limited chances of long-term success.

- The facility might benefit from a formalised management safety contact scheme, of the type used by organisations such as DuPont. This should complement the efforts with behavioural safety, demonstrate management interest and commitment to OHS, and provide a source of valuable intelligence on barriers to safety as perceived by the workforce. 


\section{SUMMARY AND RECOMMENDATIONS}

- The figure below shows the relative strengths of the four elements that have featured in this exercise. The scores have been derived largely from responses from the site OHS representative and the verification activities that went with this. Collectively, they represent an indirect indicator of innovation, activity and commitment at the site and should carry some predictive potential in terms of OHS outcomes.

- The scores indicate that the facility is meeting its statutory obligations quite well and, additionally, has several "beyond compliance" initiatives in place.

- The process has identified 16 potential areas for benchmarking (9 in the organisation of work area, 4 in the workplace environment area and 3 in the people area. In addition, there are strong efforts being applied in meeting regulatory requirements and some of the work being done there may be of interest to a benchmarking partner.

- The culture at the site appears to be open to exchange of ideas and there should be value in pursuing benchmarking opportunities - within the wider organisation to which the facility belongs and with other mine sites that are showing interest in this approach.

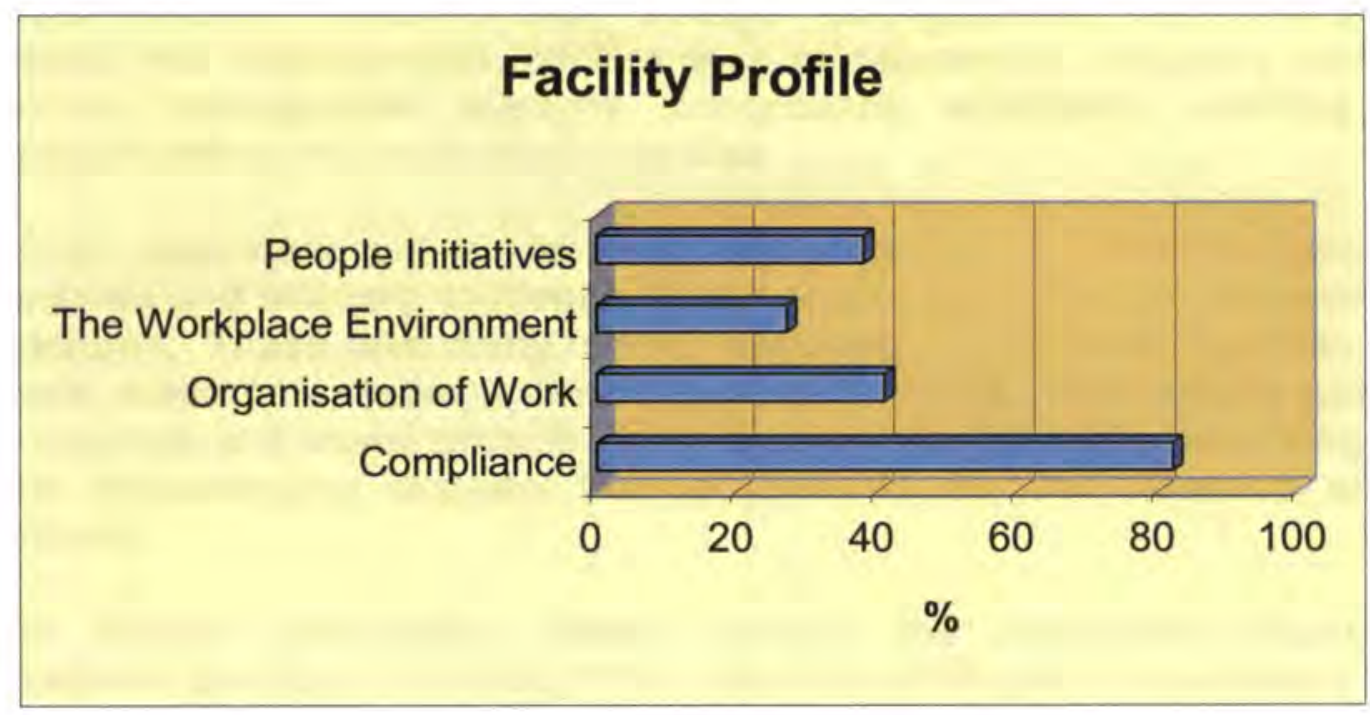

Figure 1: Profile for Sparkles Gold Mine 


\section{ABOUT THIS PROJECT}

* This research forms part of PhD studies being undertaken by Barry Chesson, Manager - Occupational Hygiene, Alcoa World Alumina Australia. The studies are being pursued though the Faculty of Communications, Health and Science at Edith Cowan University (Joondalup Campus).

* The full study title is "In Pursuit of Best Practice: Benchmarking Tools and Processes for the Management of Hazardous Substances in the Workplace."

* This study has sought to develop and test new tools and processes to make benchmarking activity and the transfer of technology, ideas and approaches more efficient and meaningful. It has focused on the management of chemicals within the Westem Australian Mining Industry, but outcomes should be applicable within the wider framework of OHS and to other industry sectors.

* It has drawn heavily from state-of-the-art safety management theory and has sought to establish the linkage between the organisation of work factor, the workplace environment factor and the people factor as they contribute to workplace health and safety performance.

* Six booklets have been used to assist with the gathering of data for this project. Content taps into current thinking and practice with respect to quality management and continuous improvement, organisational effectiveness, change management, behavioural safety, risk management, performance measurement, reporting and review, management systems, compliance, standards, auditing, benchmarking and technology transfer.

* Study outcomes will assist with the selection of benchmarking partners and will help to identify "pockets of excellence" for focused attention. These take many forms - variously as products, services, tools, resources, systems, concepts or approaches. This activity will encourage and assist organisations to take steps towards identifying and implementing Industry best practice in the OHS element of interest.

* For further information please contact the researcher, Barry Chesson, on (08) 94103301 (W) or $\quad$ or on e-mail at barry.chesson@aicoa.com.au. 
APPENDIX 9

\section{SUPPLEMENTARY EXERCISE ON TRANSFERABILITY - A BOOKLET FOR ERGONOMICS -}




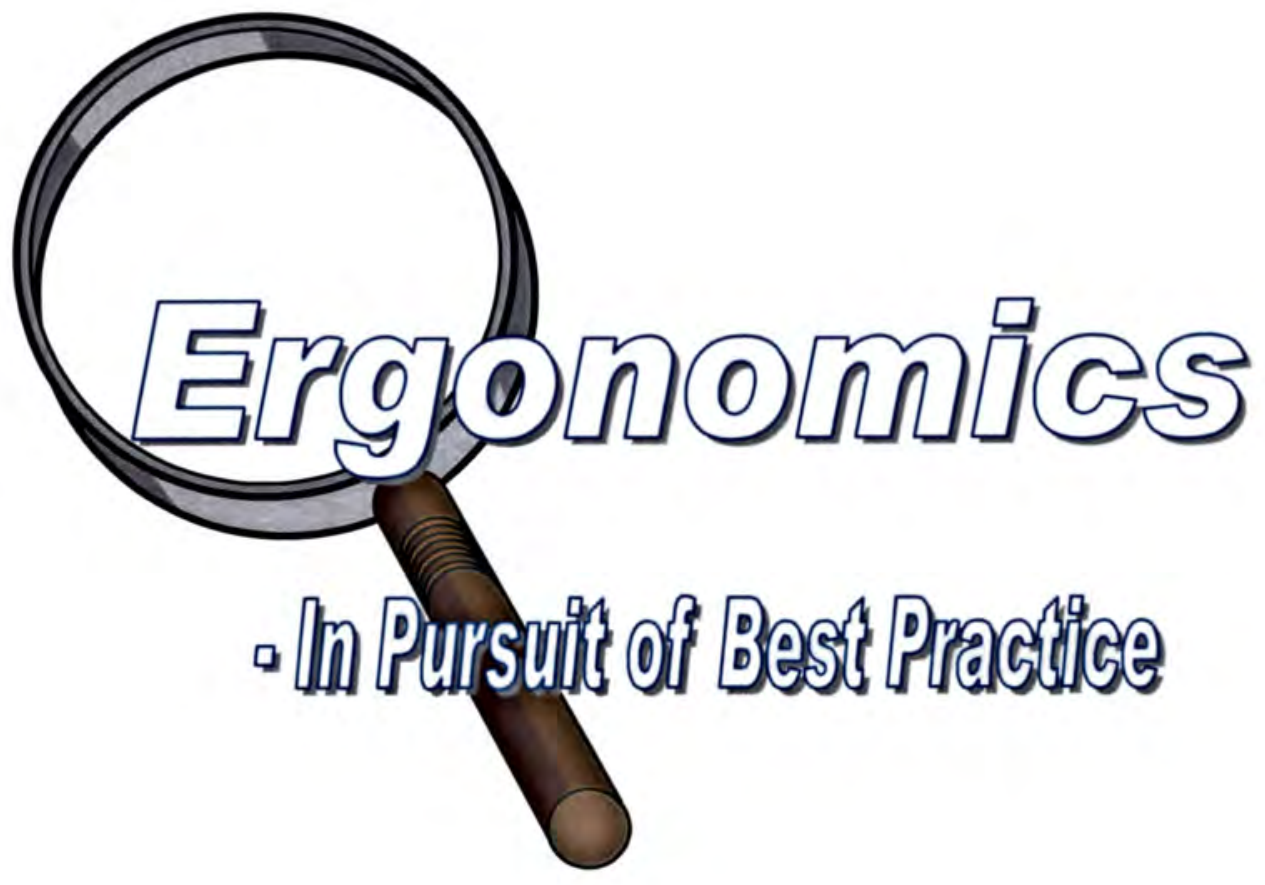

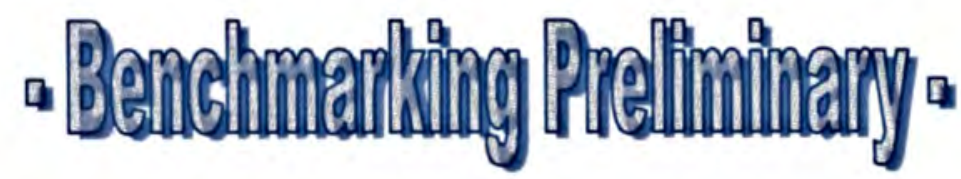

Barry Chesson

February 2003 
This booklet is designed to stimulate discussion and gather information on the organisational, technical and people measures that the site applies to the management of ergonomic risk. The purpose is to identify innovative, cost effective and practical approaches to addressing ergonomic risks ... those that go beyond the requirements expressed in law and have potential to be used in a two-way exchange between benchmarking partners.

SITE:

CONTACT PERSON:

\section{PART A: ERGONOMICS - GENERAL}

This Part is used to establish what program elements are in place at the facility and to establish potential areas for more in-depth discussion.

\section{A1 Yes/No Questionnaire}

1. Does your location have a written ergonomics (or manual handling) action plan or strategy?

Yes No

2. Have ergonomic principles been incorporated into the accident investigation process, especially for over-exertion injuries and illness?

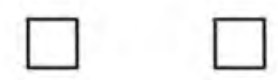

3. Is management support communicated through words, actions and resource allocation?

4. Has injury/illness data analysis been used to assist in identification of potential areas of ergonomic need?

5. Has a group or person been identified to lead the ergonomic effort and are their roles and responsibilities clearly established? 
6. Have the majority of engineers and designers at the site received basic ergonomic training?

7. Is ergonomics an integral part of discussions at the project design stage?

8. Have ergonomic opportunities at the facility been identified, assessed and prioritised?

9. Have teams or workgroups been established to address eigonomic issues or concerns?

10. Has awareness training been provided to employees exposed to ergonomic risk factors?

11. Is there encouragement of early reporting of the initial signs or symptoms of over-exertion injuries or illnesses?

12. Are the facility's ergonomic activities generally well documented?

13. Are all new or modified items of equipment and processes reviewed for ergonomic risk?

14. Are there periodic internal audits of the ergonomic program with feedback to sponsors?

15. Is there a strategy in place to encourage employee involvement and to respond effectively to their contribution?

16. Have measurements been established to monitor the effectiveness of the ergonomic program?

17. Is there an effective medical management system to help with diagnosis, treatment and prevention of over-exertion injuries? 


\section{A.2 Critical Success Factors}

What do you consider to be the four most critical success factors in relation to management of ergonomic risk at your site?

1.

2.

3.

4.

\section{A.3 Measurement}

How do you measure performance in relation to these critical success factors?

\section{A.4 Innovations}

What innovations in the ergonomics area have been introduced to your site? In other words, what do you do in this area that is different (and better) than other sites you have seen or heard about? 


\section{A.5 Flow Chart}

Please show the steps taken after a significant ergonomic risk is identified at your location. 


\section{PART B: ERGONOMICS - RATING OF PROGRAM ELEMENTS}

This Part is used for self-rating of program elements that are strongly advocated in the mainstream safety literature. Where the interviewee assigns a rating of "Good" or "Excellent", there is a follow-up question about what initiatives give rise to this opinion. This approach helps to flush out "pockets of excellence" and potential benchmarking opportunities. "Poor" is circled where the element is absent at the facility and a "Fair" rating is assigned where the element has been applied sporadically or is at an early stage of development. There are no follow-up questions when "Poor" or "Fair" are nominated.

\section{B.1 Management Leadership \& Employee Participation}

1. Please rate management visibility and activity in ergonomics.

\begin{tabular}{|l|l|l|l|}
\hline Poor & Fair & Good & Excellent \\
\hline
\end{tabular}

If "Good" or "Excellent", what are the key initiatives or success factors in your approach?

2. Please rate integration of ergonomics into business planning processes at the facility.

\begin{tabular}{|l|l|l|l|}
\hline Poor & Fair & Good & Excellent \\
\hline
\end{tabular}

If "Good" or "Excellent", what are the key initiatives or success factors in your approach?

3. Please rate commitment of financial resources to ergonomic initiatives.

\begin{tabular}{|l|l|l|l|}
\hline Poor & Fair & Good & Excellent \\
\hline
\end{tabular}

If "Good" or "Excellent", what are the key initiatives or success factors in your 
approach?

4. If a written ergonomic program exists, please rate it in terms of its outline of objectives, leadership, scope, responsibilities and ergonomic initiatives.

\begin{tabular}{|c|c|c|c|}
\hline Poor & Fair & Good & Excellent \\
\hline
\end{tabular}

If "Good" or "Excellent", what are the key initiatives or success factors in your approach?

5. Please rate efforts and arrangements to encourage employee participation and active involvement in the implementation, evaluation and continuous improvement of the ergonomics program.

\begin{tabular}{|c|c|c|c|}
\hline Poor & Fair & Good & Excellent \\
\hline
\end{tabular}

If "Good" or "Excellent", what are the key initiatives or success factors in your approach?

\section{B.2 Ergonomic Risk Factor Evaluation}

1. Please rate processes by which employees can report ergonomic concerns and/or suggestions to the group handling ergonomics, and receive a timely response.

\begin{tabular}{|c|c|c|c|}
\hline Poor & Fair & Good & Excellent \\
\hline
\end{tabular}

If "Good" or "Excellent", what are the key initiatives or success factors in your approach? 
2. Please rate tools and processes to identify risks that require an ergonomic hazard analysis (these include hazard checklists, job observations, accident/incident logs, plant medical records, safety reports, consultation with employees, and similar.

\begin{tabular}{|c|c|c|c|}
\hline Poor & Fair & Good & Excellent \\
\hline
\end{tabular}

If "Good" or "Excellent", what are the key initiatives or success factors in your approach?

3. Please rate access to external resources for ergonomic risk factor evaluation.

\begin{tabular}{|l|l|l|l|}
\hline Poor & Fair & Good & Excellent \\
\hline
\end{tabular}

If "Good" or "Excellent", what are the key initiatives or success factors in your approach?

\section{B.3 Ergonomic Hazard Analysis \& Control}

1. Please rate efforts to analyse jobs or tasks where work-related musculoskeletal symptoms or disorders have been reported.

\begin{tabular}{|l|l|l|l|}
\hline Poor & Fair & Good & Excellent \\
\hline
\end{tabular}

If "Good" or "Excellent", what are the key initiatives or success factors in your approach?

2. Please rate efforts to perform proactive analyses of jobs and tasks where concerns exist about ergonomic risk.

\begin{tabular}{|l|l|l|l|}
\hline Poor & Fair & Good & Excellent \\
\hline
\end{tabular}


If "Good" or "Excellent", what are the key initiatives or success factors in your approach?

3. Please rate efforts to perform analyses prior to adopting a change in process, equipment, work practices, division of labour or work schedule (to prevent introduction of new ergonomic risks).

\begin{tabular}{|l|l|l|l|}
\hline Poor & Fair & Good & Excellent \\
\hline
\end{tabular}

If "Good" or "Excellent", what are the key initiatives or success factors in your approach?

4. Please rate priority-setting tools and arrangements (for control of ergonomic risks).

\begin{tabular}{|l|l|l|l|}
\hline Poor & Fair & Good & Excellent \\
\hline
\end{tabular}

If "Good" or "Excellent", what are the key initiatives or success factors in your approach?

5. Please rate the content of the tool used at the facility for Ergonomic Hazard Analysis (eg. its assessment of force, repetition, posture, vibration, contact stress and similar factors).

\begin{tabular}{|c|c|c|c|}
\hline Poor & Fair & Good & Excellent \\
\hline
\end{tabular}

If "Good" or "Excellent", what are the key initiatives or success factors in your approach? 
6. Please rate efforts to develop and apply action plans to reduce or eliminate each risk, with appropriate timeframes.

\begin{tabular}{|c|c|c|c|}
\hline Poor & Fair & Good & Excellent \\
\hline
\end{tabular}

If "Good" or "Excellent", what are the key initiatives or success factors in your approach?

\section{B.4 Education, Training \& Communication}

1. Please rate the quality and effectiveness of ergonomics training as it is applied at induction.

\begin{tabular}{|l|l|l|l|}
\hline Poor & Fair & Good & Excellent \\
\hline
\end{tabular}

If "Good" or "Excellent", what are the key initiatives or success factors in your approach?

2. Please rate the quality and effectiveness of on-going, basic ergonomic training provided for management and employees at the facility.

\begin{tabular}{|c|c|c|c|}
\hline Poor & Fair & Good & Excellent \\
\hline
\end{tabular}

If "Good" or "Excellent", what are the key initiatives or success factors in your approach?

3. Please rate the quality and effectiveness of training in ergonomic design principles as may be provided to those responsible for the design or modification of work stations, tools, equipment and/or work processes.

\begin{tabular}{|l|l|l|l|}
\hline Poor & Fair & Good & Excellent \\
\hline
\end{tabular}


If "Good" or "Excellent", what are the key initiatives or success factors in your approach?

4. Please rate arrangements to communicate program contents, objectives, initiatives and results to employees.

\begin{tabular}{|l|l|l|l|}
\hline Poor & Fair & Good & Excellent \\
\hline
\end{tabular}

If "Good" or "Excellent", what are the key initiatives or success factors in your approach?

\section{B.5 Management of Work-Related Musculoskeletal Disorders}

1. Please rate the arrangements at the facility to investigate reports of musculoskeletal complaints and disorders regarding work-relatedness, contributing ergonomic risk factors and potential corrective action.

\begin{tabular}{|l|l|l|l|}
\hline Poor & Fair & Good & Excellent \\
\hline
\end{tabular}

If "Good" or "Excellent", what are the key initiatives or success factors in your approach?

2. Please rate the arrangements to provide employees with prompt access to a health care professional for evaluation, management and follow-up.

\begin{tabular}{|l|l|l|l|}
\hline Poor & Fair & Good & Excellent \\
\hline
\end{tabular}

If "Good" or "Excellent", what are the key initiatives or success factors in your approach? 
3. Please rate the arrangements for servicing by external health care providers (where there are no on-site medical personnel).

\begin{tabular}{|l|l|l|l|}
\hline Poor & Fair & Good & Excellent \\
\hline
\end{tabular}

If "Good" or "Excellent", what are the key initiatives or success factors in your approach?

4. Please rate the arrangements and mechanisms for return to work of employees who have been injured and/or assigned work restrictions.

\begin{tabular}{|l|l|l|l|}
\hline Poor & Fair & Good & Excellent \\
\hline
\end{tabular}

If "Good" or "Excellent", what are the key initiatives or success factors in your approach?

\section{B.6 Ergonomics Record Keeping}

1. Please rate arrangements for recording risk factors for different tasks/jobs, controls implemented and the degree of success of those controls.

\begin{tabular}{|c|c|c|c|}
\hline Poor & Fair & Good & Excellent \\
\hline
\end{tabular}

If "Good" or "Excellent", what are the key initiatives or success factors in your approach?

2. Please rate arrangements for recording the ergonomics training that has been offered and employee attendance at training.

\begin{tabular}{|c|c|c|c|}
\hline Poor & Fair & Good & Excellent \\
\hline
\end{tabular}


If "Good" or "Excellent", what are the key initiatives or success factors in your approach?

3. Please rate arrangements for recording the results of any risk assessments that have been carried out.

\begin{tabular}{|l|l|l|l|}
\hline Poor & Fair & Good & Excellent \\
\hline
\end{tabular}

If "Good" or "Excellent", what are the key initiatives or success factors in your approach?

\section{B.7 Ergonomics Program Evaluation \& Measurement}

1. Please rate the use of "lagging" indicators of ergonomic performance on-site.

\begin{tabular}{|c|c|c|c|}
\hline Poor & Fair & Good & Excellent \\
\hline
\end{tabular}

If "Good" or "Excellent", what are the key initiatives or success factors in your approach?

2. Please rate the use of "leading" indicators of ergonomic performance on-site.

\begin{tabular}{|l|l|l|l|}
\hline Poor & Fair & Good & Excellent \\
\hline
\end{tabular}

If "Good" or "Excellent", what are the key initiatives or success factors in your approach? 
3. Please rate the arrangements to evaluate the effectiveness of ergonomic efforts and to track achievements against Corporate objectives.

\begin{tabular}{|l|l|l|l|}
\hline Poor & Fair & Good & Excellent \\
\hline
\end{tabular}

If "Good" or "Excellent", what are the key initiatives or success factors in your approach?

\section{B.8 Technology Transfer - Ergonomics}

1. Please rate arrangements at the site to identify ergonomics-related transferable technology.

\begin{tabular}{|l|l|l|l|}
\hline Poor & Fair & Good & Excellent \\
\hline
\end{tabular}

If "Good" or "Excellent", what are the key initiatives or success factors in your approach?

2. Please rate processes and practices at the site with regard to communication of ergonomics projects, initiatives, problems and solutions across the facility and within the wider organisation.

\begin{tabular}{|l|l|l|l|}
\hline Poor & Fair & Good & Excellent \\
\hline
\end{tabular}

If "Good" or "Excellent", what are the key initiatives or success factors in your approach? 
* This research forms part of PhD studies being undertaken by Barry Chesson, Manager - Occupational Hygiene, Alcoa World Alumina Australia. The studies are being pursued though the Faculty of Communications, Health and Science at Edith Cowan University (Joondalup Campus).

* The full study title is "In Pursuit of Best Practice: Benchmarking Tools and Processes for the Management of Hazardous Substances in the Workplace."

* This study has sought to develop and test new tools and processes to make benchmarking activity and the transfer of technology, ideas and approaches more efficient and meaningful. It has focused on the management of chemicals within the Western Australian Mining Industry, but outcomes should be applicable within the wider framework of OHS and to other industry seciors.

- It has drawn heavily from state-of-the-art safety management theory and has sought to establish the linkage between the organisation of work factor, the workplace environment factor and the people factor as they contribute to workplace health and safety performance.

* Six booklets have been used to assist with the gathering of data for this project. Content taps into current thinking and practice with respect to quality management and continuous improvement, organisational effectiveness, change management, bohavioural safety, risk management, performance measurement, reporting and review, management systems, compliance, standards, audiring, benchmarking and technology transfer.

- Study outcomes will assist with the selection of benchmarking partners and will help to identify "pockets of excellence" for focused attention. These take many forms - variously as products, services, tools, resources, systems, concepts or approaches. This activity will encourage and assist organisations to take steps towards identifying and implementing Industry best practice in the OHS element of interest.

* For further information please contact the researcher, Barry Chesson, on (08) 94103301 (W) or $\quad$ or on e-mail at barry.chesson@alcoa.com.au. 


\section{BENCHMARKING OPPORTUNITIES}

- "Top Four" Benchmarking Opportunities - OHS (General)

- "Top Four" Benchmarking Opportunities - Chemical Safety 


\section{Facility Principal Benchmarking Opportunities}

1 - Strategic and tactical planning templates.

- Employee involvement and communication initiatives.

- Feasibility assessments for engineering controls.

- A Performance Support System (controlled documents covering hundreds of procedures and administrative arrangements).

- Integration of site and departmental plans with those of the broader Business Unit.

- Induction training, built around a one-day MARCSTA program, followed by a one-day Company program and a half-day departmental program.

- An organisational structure that encourages interaction of OHS professionals with line management and shop floor personnel.

- Delivery of feedback via "toolbox" meetings, electronic and hard copy reports, newsletters and presentations.

- The facility has a very strong emphasis on firstaid training and qualifications.

- There is a comprehensive site induction program - utilising a computer-based training module, an in-house induction video and a competency questionnaire.

- Communication of OHS targets is via publication of monthly statistics, a bi-monthly HSE Newsletter, published notices, computerbased reporting systems and delivery to employee work groups and committees.

- The Company has developed an "Integrated Risk Management System" which seeks to integrate HSE and Quality into an overall approach to risk management. 
- Strategic planning with very structured Key Performance Indicators.

- Deployment of the B-Safe Safety Management System.

- Deployment of risk management activities built around monthly inspection reports, daily workplace inspections, task observations and pre-start checks.

- Safety initiatives to effect cultural change and behavioural change.

- The Company's administrative management system.

- Well-developed reporting protocols.

- Site induction training programs based on CDRom tools.

- Accident investigation systems and tools.

- Deployment of the Drugs and Alcohol Program. This covers pre-employment, "for cause" and random testing aspects.

- Deployment of the Safe Act Observation Program.

- Catastrophic risk management.

- Deployment of the facility's IH program. There is a risk-based sampling component, supported by statistical analysis software.

- Deployment of the organisation's Safety Management System.

- Application of the "Whole of Mining Risk Assessment".

- Accident investigation protocols and their application.

- Emergency response procedures and facilities.

- Written procedures for management of change.

- Use of an "approved mentor" system with contractors.

- Use of "Six Sigma" and "Blitz" approaches to finding solutions and establishing boundaries.

- Application of the organisation's data management system. 
"TOP FOUR" BENCHMARKING OPPORTUNITIES - CHEMICAL SAFETY

\begin{tabular}{|c|c|}
\hline Facility & Principal Benchmarking Opportunities \\
\hline 1 & $\begin{array}{l}\text { - Use of "Hygenius" software for qualitative and } \\
\text { quantitative exposure assessment. } \\
\text { - Comprehensive procedures for vetting new } \\
\text { materials and controlling chemical use on site. } \\
\text { Use of Job Dictionaries and Similar Exposure } \\
\text { Groups to define exposure characteristics } \\
\text { associated with all jobs and tasks on the site. } \\
\text { - Use of chemical inventories in training, } \\
\text { awareness-building and auditing applications. }\end{array}$ \\
\hline 2 & $\begin{array}{l}\text { - Exposure baselines have been developed for all } \\
\text { Similar Exposure Groups. } \\
\text { - Software provides for long-term storage of } \\
\text { electronic records, while hard copy IH-related } \\
\text { records are stored in secure, off-site archives. } \\
\text { - A "Safety Dashboard" is applied to review } \\
\text { progress against objectives for chemical safety. } \\
\text { - Hazard communication is delivered via } \\
\text { Significant Incident Reports, toolbox talks, } \\
\text { Safety Alerts, feedback after air sampling } \\
\text { exercises, use of Chemweb MSDS database, } \\
\text { awareness training and similar. }\end{array}$ \\
\hline
\end{tabular}

- Qualitative assessments are conducted via a program described in the SHE Policy \& Procedures Manual.

- Introduction of a PPE eye protection upgrade initiative for dusty environments.

- Hazard communication initiatives. These are built around training programs, risk assessment activities, procedures, signage, labels and hazard area diagrams.

- There are several examples of successful substitution and elimination initiatives relating to chemical safety. 
- Use of a portable, mobile equipment bulk underground re-fuelling facility.

- Supply of non-toxic and environmentallyfriendly degreasing products.

- Training modules for the storage, use, handling and transportation of explosives.

- Bulk supply of chemicals such as fuels and explosives.

- Use of the Chem Alert materials information service.

- Deployment of procedures relating to use of chemicals and control of the generation of airborne contaminants.

- Use of a system to address ordering, vetting, supply and distribution of hazardous materials.

- Deployment of personal protective equipment systems and procedures.

- Baseline sampling for dusts and other particulates.

- Check sheets for assessing quality and sufiiciency of information provided on MSDSs.

- Vetting arrangements for processing requests for new chemicals.

- Annual inspection and audit program.

- Use of the Chem Alert database to assist with the management of hazardous materials.

- Permits to work, procedures and training associated with work in confined spaces.

- Deployment of the chemical audit program.

- Vetting arrangements and controls for new chemicals.

- Use of the Hazardous Materials Risk Assessment process.

- Deployment of hazard information to the workforce and contractors.

- Use of confined space permit to work systems.

- Use of Chem Alert materiais to convey hazard information to the workforce. 